\title{
Decision-making and Strategic Management as Sources of Sustained Competitive Advantage in a High Cost Private Multi-campus University in México
}

\author{
CORVINUS UNIVERSITY OF BUDAPEST \\ Ph.D. in Management and Business Administration \\ José Francisco Enríquez de la 0
}

Supervisor: Professor Károly Balaton, Ph.D.

September 30th, 2016. 
To my lovely wife and my daughter, for supporting and joining me on this amazing adventure

To my parents and brothers, who taught me the value of effort and hard work 


\section{Abstract}

Higher education institutions (from now on HEI) are facing a tremendous and complex environment nowadays, due to the changes that this sector has experimented in the last years. Strategy in HEI is dynamic and there are several variables to consider when developing a strategy that can achieve sustained competitive advantage in HEI: academic quality, professors, relationship with industry, enrollment of students, research and development, satisfaction of students, position among competitors, new technologies in education, facilities, good services, the best educative technology, finances, among others.

Decision-makers will have to make strategic decisions surrounded by risk and uncertainty, and their experience, skills and the way they manage risk will be key in the correct decision-making process. During the execution of their strategies, they also will have to struggle with the assignation of their limited time and resources between exploitative activities, those focused on the day to day operation, and the explorative activities, those focused on finding new opportunities and goals that could provide competitive advantages in the long term, which in turn could differentiate their institutions.

This dissertation follows a qualitative phenomenological exploratory approach to study how sample directors behave regarding risk and uncertainty in decision-making and regarding ambidexterity, and how risk in decision-making and ambidexterity affect the performance in their organizations. The findings suggest specific behaviors of the directors regarding risk in DM and ambidexterity, and that those behaviors are related to the strategy, the performance of their organizations and the deployment of dynamic capabilities. Finally, this dissertation suggests directions for future research and implications for management practice.

Keywords: Strategy, ambidexterity, decision-making, risk, uncertainty, exploitation, exploration, dynamic capabilities, resource- based view. 


\section{ACKNOWLEDGMENTS}

First, I would like to thank God, for giving me the opportunity to combine the process of the PhD with my family, and I would like to thank my family, my wife Paulina for her amazing support in these last years, for being my partner in this challenging trip. To my beautiful daughter Paulinita, for being my company, my inspiration and my driver in the last two years, who pushed me to keep working hard and never desisting. Thank you both for your love and patience.

Special thanks to my parents, Maria del Rosario and Javier, who encouraged me to seize all opportunities that life brings on, including the enrollment to the PhD. For their advices and for being my example of tenacity, persistence and hard work, and for teaching me the meaning of leadership. To my brothers, Javier and Adrian, who were always there, giving their example and supporting me on those difficult moments that made me consider the possibility to give up.

I want also thanks to a boss, colleague but overall a friend, Javier Quezada, who was part of my decision to study my PhD. For being an example of how a good strategist, leader and friend should behave. For teaching me that in this life it is worth to dream. I want to thank to Alfonso Pompa, who was the person that invited me to consider the importance of studying a $\mathrm{PhD}$, and who gave me that possibility; thanks also for being an inspirational leader. Thank you to all my friends and all the persons that were part of this process, each of them who invite me to go on and to finish this goal. Thank to Eva, a very important person that helped me with her knowledge and experience, thanks for her invaluable advices; and thanks to Professor Myrta

Rodríguez who also helped me with her advices and experience. Thanks to all my team, for taking care of that and supported me in those moments that PhD demanded me a lot of time.

To finalize, I want to thank a very special and important person that have influenced my professional life in the last 5 years; a person who stands out for his humility, 
despite being a great teacher and researcher and even a better person. I am referring to Professor Károly Balaton. Thanks Karoly for your advices, for giving me your valuable time and experience, for your patience and for all what I have learned from you. 


\section{ABBREVIATIONS AND ACRONYMS}

HEI

Higher Education Institutions

$\mathrm{DM}$

Decision making

SCA

Sustained Competitive Advantage

DC

Dynamic Capabilities

RBV

Resourced-based View

VRIN

Valuable, Rare, In-imitable and Non-substitutable

EAGLE

Emerging And Growth-Leading Economies

FIMPES

Private Higher Education Mexican Institutions Federation, FIMPES, for its acronym in Spanish

KPI

Key Performance Indicators

PDM

Participative Decision Making

iWeek

Innovation week. It is an innovative element in the new Educative Model at Tecnológico de Monterrey.

iSemester Innovation semester. It is an innovative element in the new Educative Model at Tecnológico de Monterrey.

Tecnológico de Monterrey

Refers to the private multi-campus university to which the selected campuses for the research belong. 


\section{TABLE OF CONTENTS}

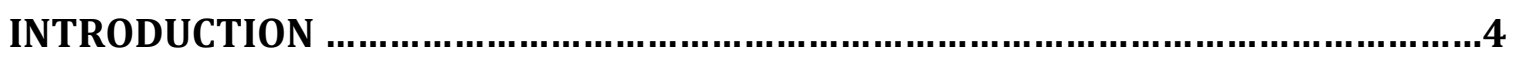

1 CHAPTER 1. CONTEXT, PROBLEM DEFINITION, RESEARCH QUESTION, OBJECTIVES, JUSTIFICATION, AND LIMITATIONS. ............................................. 18

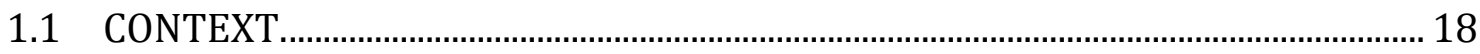

1.1.1 Some Precedents about Tecnológico de Monterrey University........................................ 21

1.1.2 Context of Some Multi-campus Universities in México ……............................................. 22

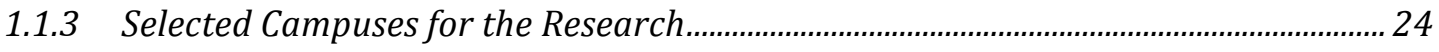

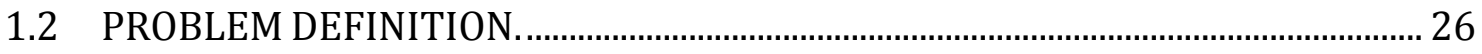

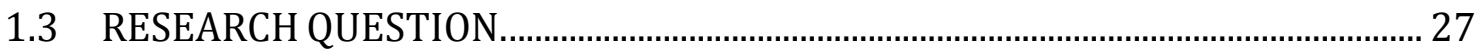

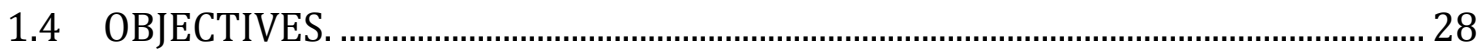

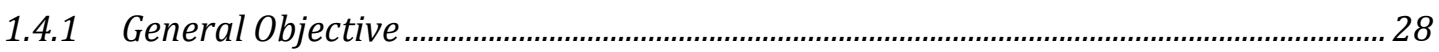

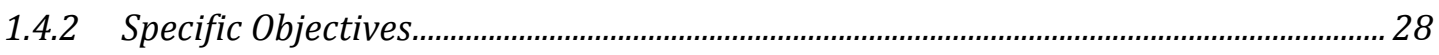

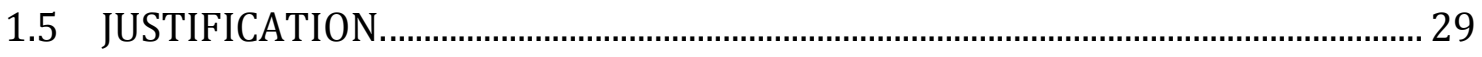

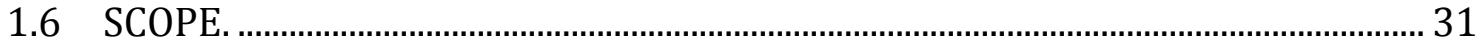

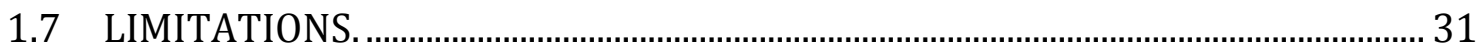

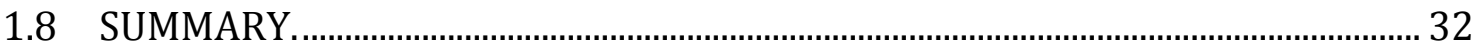

2 CHAPTER 2. LITERATURE REVIEW.................................................................. 33

2.1 THE IMPORTANCE OF STRATEGIC MANAGEMENT: BASIC CONCEPTS ABOUT

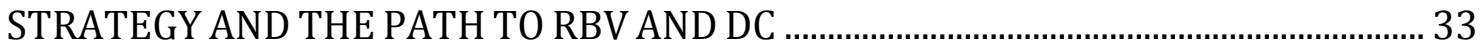

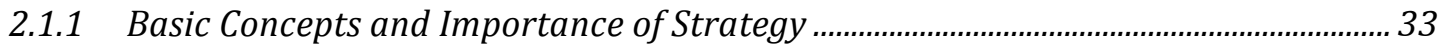

2.1.2 Considerations about Strategic Management Process and Environment................... 39

2.1.3 Considerations about Strategic Formulation and Execution ........................................ 41

2.2 RESOURCE-BASED VIEW, DYNAMIC CAPABILITIES AND AMBIDEXTERITY: ACHIEVING COMPETITIVE ADVANTAGE THROUGH INTERNAL RESOURCES AND

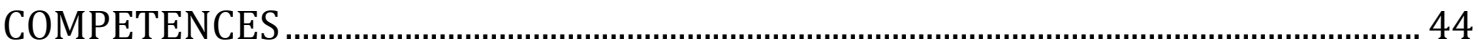

2.2.1 UNDERSTANDING RESOURCE-BASED VIEW AND DYNAMIC CAPABILITIES........... 45

2.2.1.1 Toward a Dynamic Capabilities approach from Resource-Based View ........................... 48 


\subsubsection{RESOURCE BASED VIEW AND DYNAMIC CAPABILITIES AS SOURCES OF PROFIT}

AND SUSTAINED COMPETITIVE ADVANTAGE. 52

\subsubsection{EXEMPLIFYING APPLIED STRATEGY BASED IN RESOURCE-BASED VIEW AND}

DYNAMIC CAPABILITIES. 55

2.2.3.1 Role of managers in resourced-based view and dynamic capabilities strategies.......56

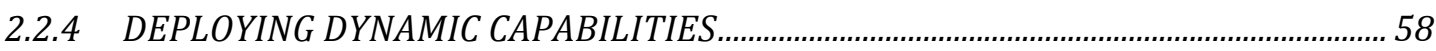

2.2.4.1 Understanding the creation of dynamic capabilities...........................................................58

2.2.4.2 Examples of dynamic capabilities ............................................................................................. 59

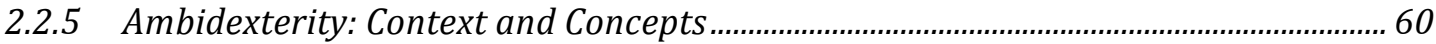

2.2.5.1 What Exploration and Exploitation means from the Context of Ambidexterity..........61

2.2.5.2 Different ways of Achieving Ambidexterity in the Organizations ....................................63

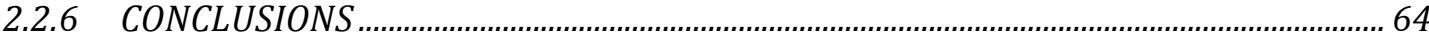

\subsection{INDIVIDUAL DECISION MAKING BY TOP EXECUTIVES AS A VALUABLE} RESOURCE FOR STRATEGIC MANAGEMENT: A RESOURCE-BASED VIEW AND

2.3.1 STRATEGIC DECISION MAKING: CHALLENGES OF INDIVIDUAL DM............................ 68

2.3.1.1 Individual Decision-Making in Controlled Sceneries ............................................................69

2.3.1.2 Decision-Making in Ambiguous and Complex Sceneries ...................................................70

2.3.1.3 Rational vs non- rational: Decision-makers limited by their bounded rationality.....71

2.3.2 DECISION MAKING IN MEDIUM AND HIGH LEVEL POSITIONS................................... 74

2.3.2.1 Distortion of biases when making decisions .......................................................................75

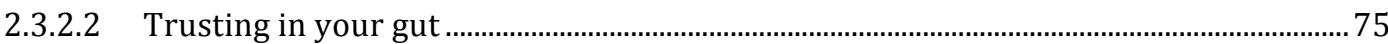

2.3.3 INDIVIDUAL DECISION MAKERS AS VRIN RESOURCES AND ENABLERS OF DC: ACHEIVING PERFORMANCE THROUGH DECISION-MAKING ................................................. 78

2.3.3.1 Top Executive Decision Makers as VRIN resources ................................................................. 78

2.3.3.2 Top Executive Decision Makers as enablers of Dynamic Capabilities ...............................80

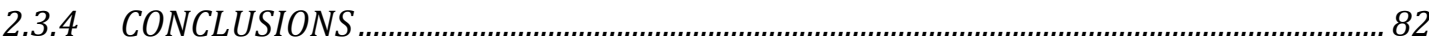

2.4 STRATEGIC MANAGEMENT IN HIGHER EDUCATION INSTITUTIONS .............. 84

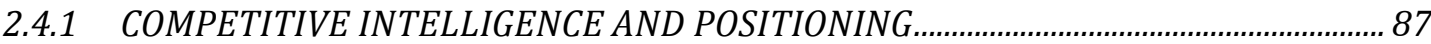

2.4.1.1 Confronting planning versus action in Strategic Management in HEI ............................... 88

2.4.2 ORGANIZATIONAL STRUCTURE AND THE TRAP OF COPYING INCORRECT MODELS

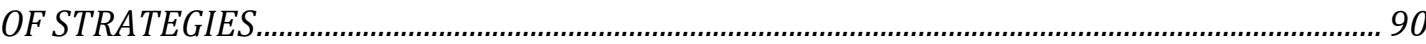

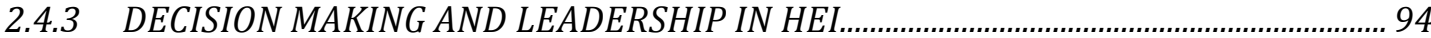

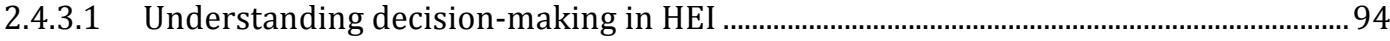

2.4.3.2 The challenge of leadership in HEI .......................................................................................... 99 
2.4.3.3 Academic Leadership or Management

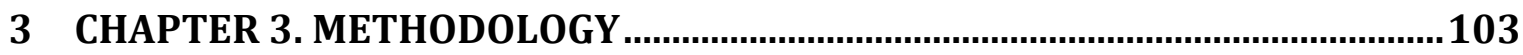

3.1 APPROACH ……………………........................................................................103

3.2 RESEARCH STRATEGY …………………………......................................................103

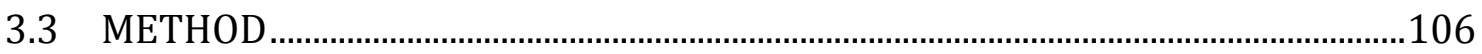

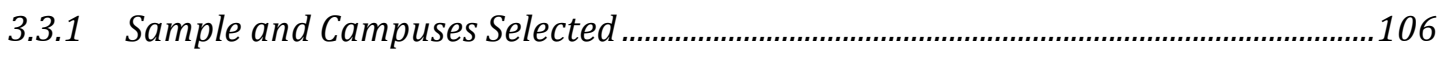

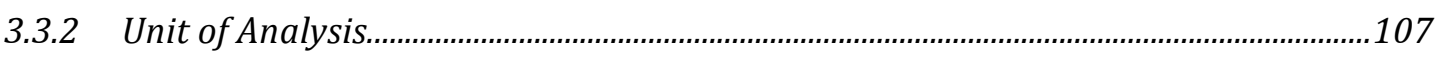

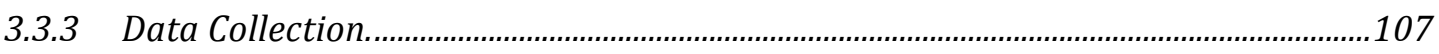

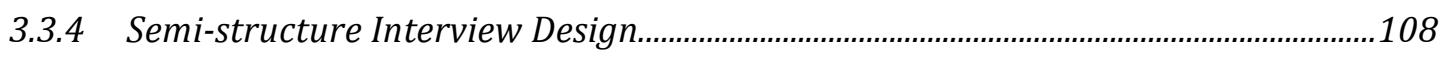

3.3.5 Methodology Procedure to address the Research Question and Objectives............109

3.3.5.1 Collecting Data, Organizing and Preparing it for Analysis ............................................. 110

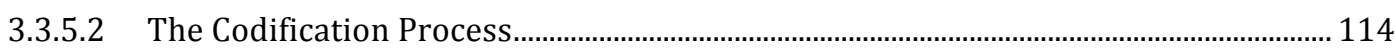

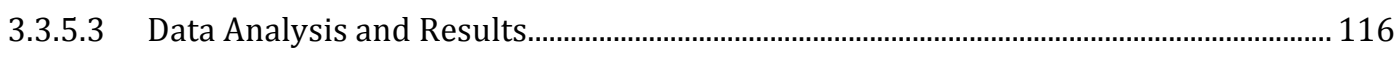

3.4 VALIDITY AND RELIABILITY ................................................................................117

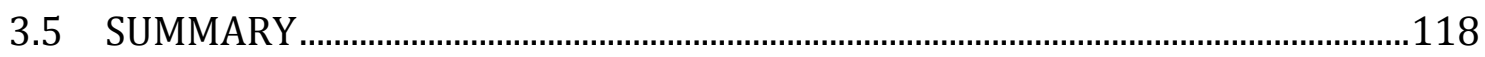

4 DATA ANALYSIS. .............................................................................................120

4.1 Analysis of Risk And Uncertainty in Decision-Making Theme...............................120

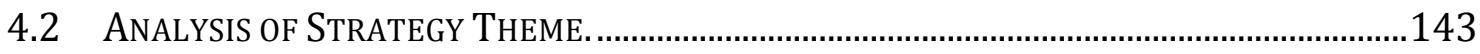

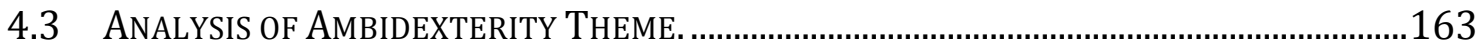

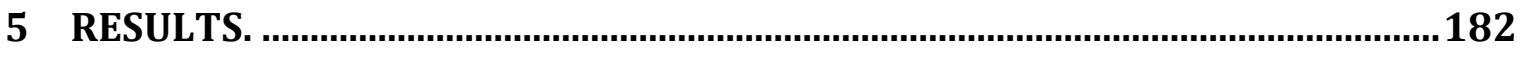

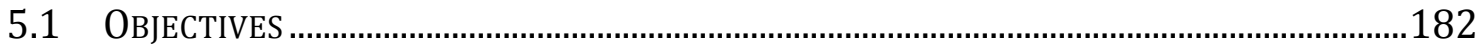

5.1.1 To understand how high level directors behave regarding to risk and uncertainty

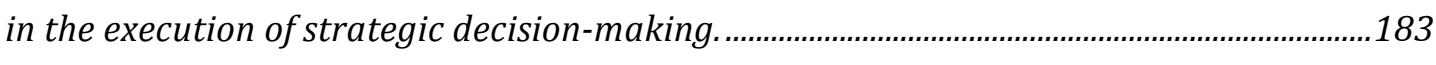

5.1.2 To understand what are the most relevant directors' factors or characteristics that promote or inhibit ambidexterity, and how it affects the organization ................................186

5.1.2.1 Emergent topics .............................................................................................................. 190

5.1.3 To Understand whether Ambidexterity and Risk, Uncertainty and Gut in DM is related with the performance and how it is affected................................................................191

5.1.3.1 How risk and uncertainty affects the performance of the organization ...................... 191

5.1.3.2 How ambidexterity affects the performance (or the strategies).................................. 193

5.1.4 To understand how directors perceived their own strategy and how is it related to

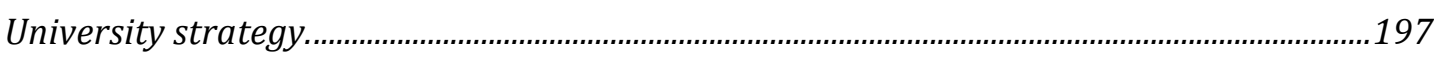


5.1.4.1 Emergent topic: Threats and Risks for the University Strategy.

5.1.5 To understand whether ambidexterity and/or risk and uncertainty in strategic DM can influence dynamic capabilities..

5.2 RESEARCH QUESTION.

5.2.1 How high level directors behave regarding ambidexterity and risk in strategic decision-making and how they are related to performance in 4 campuses of a high cost multi-campus private university in Mexico?

6 CONCLUSIONS, IMPLICATIONS AND FUTURE RESEARCH.

6.1 MANAGERIAL IMPLICATIONS

6.2 FutURE RESEARCH

7 TABLES. 223

8 FIGURES. 258

9 REFERENCES. .259 


\section{LIST OF TABLES}

Table 1 Summary of Private Multi-Campus Universities with FIMPES

accreditation in México.

Table 2 Private Universities ranked according to QS Rankings $2016 \quad 219$

Table 3 Categorization of the 26 campuses of Tecnológico de 220 Monterrey.

Table $4 \quad$ Operational Efficiency and Competitive Strategy. 220

Table 5A In-depth and Confirmatory Interviewees. 220

Table 5B Sample Selection of the In-depth and Confirmatory 221

Table 6 Guide of Questions for the in-depth semi-structured 221 interview.

Table $7 \quad$ Guide of Questions for the confirmatory semi-structured 223 interview.

$\begin{array}{lll}\text { Table } 8 & \text { General data from the interviews. } & 224\end{array}$

$\begin{array}{lll}\text { Table } 9 & \text { General Codes and Themes. } & 225\end{array}$

Table $10 \quad$ Codes, Mentions and Percentage of Mentions for Risk and 226 Uncertainty in DM Theme at Interviewee detailed.

Table 11 Codes, Mentions and Percentage of Mentions for Risk and 226 Uncertainty in DM Theme.

Table 12 Code and Extended Codes for Risk and Uncertainty in DM Theme at Interviewee detailed.

Table 13 Code and Extended Codes for Risk and Uncertainty in DM 231 Theme.

Table 14 Codes, Mentions and Percentage of Mentions for Strategy Theme at Interviewee detailed.

Table 15 Codes, Mentions and Percentage of Mentions for Strategy Theme.

Table $16 \quad$ Code and Extended Codes for Strategy Theme at Interviewee 239 detailed.

Table 17 Code and Extended Codes for Strategy Theme. 
Table 18 Codes, Mentions and Percentage of Mentions for Ambidexterity Theme at Interviewee detailed.

Table 19 Codes, Mentions and Percentage of Mentions for

244 Ambidexterity Theme.

Table 20 Code and Extended Codes for Ambidexterity Theme at 250 Interviewee detailed.

Table 21 Code and Extended Codes for Ambidexterity Theme. 


\section{INTRODUCTION}

Strategic management is a wide topic in which many scholars and enterprises have invested a huge quantity of time and economic resources. During the last three decades there have been significant developments in the field of strategy, applied for several different types of industries. Strategy has been the "holy grail" for the competitiveness of enterprises. Nevertheless, "strategy" is a common word that is widely used not only by scholars or executives, but almost everyone use it in the day to day life "we need a strategy for winning the game", "we need a strategy for getting the money", "we need a strategy for passing the course", "we need a strategy for getting more votes", "we need a strategy for losing weight" and so on. "The strategy field is replete with popular business literature on strategic management and competing prescriptions, directives and recipes with regard to successful performance" (Volberda, 2004, p. 35). For the purpose of this study, the aim of strategy will be to understand, to discover and to execute new ways of sustained competitive advantage (from now on SCA).

In general, firms obtain sustained competitive advantage in such different ways: by creating new products for undiscovered markets; by developing innovative manufacturing processes; by generating new business models, and so on. We can identify several dominant themes in the evolution of strategic management from 1950 until our times. The dominant paradigm during the late 1970's and 1980's comes with Porter who claims that firms can create defensible positions in industry according to external forces, which shape the strategy to implement. According to Grant (2010), the dominant themes in these decades were strategy as positioning (in the 1970's) and quest for competitive advantage (in the 1980's). Another approach is referred as strategic conflict approach, which uses the tools of game theory to keep rivals out of market through strategic investments, pricing strategies and control of information (Teece, Pisano, \& Shuen, 1997). 
Strategic planning, business polices, changing of competitive landscape, top level management in firms, the five competitive forces of Porter, correspond more to classical strategy perspectives (Volberda, 2004) or early and medium work in strategic management (Hitt, Gimeno, \& Hoskisson, 1998). All of the above strategy paradigms were intended to achieve SCA, but most of those approaches were based in the analysis of external environment, looking for different ways of addressing changes and looking for new opportunities of doing business and being different. But what is happening in current times? Do those approaches by their own still work for achieving SCA? Do they do for all type of enterprises? Could they be applied regardless the differences on the speed of change of different enterprises?

According to Peters (2005), we are facing a dizzying and radical change, in such way that marketing and strategic models will be obsolete in a matter of days. Yesterdays' workers are not working in today's world; yesterdays' firms are obsolete today. Everything, absolutely everything is changing at an unprecedented rate. In that context, maybe dealing only with external environment and with competitors would not be sufficient for success. Internal resources and the way they are operated and exploited should be taken into account to address such changing environment. In these conditions is where resource-base view (from now on RBV) and later dynamic capabilities (from now on DC) arise in the field of strategy. RBV of the firm allows to achieve SCA through the correct combination and use of VRIN resources (deeper information about VRIN resources and RBV will be provided in Section 1.2). Meanwhile, Dynamic Capabilities permit to develop new forms of competitive advantage through the renovation of based resources. Dynamic capabilities follow the theory of RBV of the firm (Barney, 1991), and are necessary in turbulent environments where VRIN resources provide SCA for a short period of time (deeper information about dynamic capabilities will be provided in Section 1.2).

The higher education institutions (from now on HEI) are facing a tremendous and complex environment nowadays, due to the changes that this sector has experimented in the last years (Yielder and Codling, 2004; Tavernier, 2005; 
Timberlake, 2004). As Barret (2010) points out, in universities, seeking sources of revenue has become a constant interest, and identifying opportunities for growth and strategic positioning is now essential for survival. In this same line, Washburn (2005, p. 204) claims that in last years students are referred to as "costumers", and courses as products; moreover, "even university presidents were chosen for their ability to raise money and their close ties to the corporate sector". The current complicated situation of universities is very well pointed out by Tavernier (2005, p. 2), who points out that "looking at today's environment, universities must realize that the old years are gone and never will come back. They now live in a completely different world with different requirements".

Defining appropriate strategic planning and management models in Higher Education Institutions (HEI) have become very important in the last years. In recent years most institutions have embarked upon one or other form of strategic management. But the strong competence and the demand from complex environments have made that sometimes results were below expectations. Two main reasons could be significant in those failed cases: on the one hand the lack of "identity" in the development of the strategy, falling in the temptation to copy other models mainly used for industry instead of developing "tailor made" strategic plans (Lorange \& Vancil, 1976). And the second and the most important for this study, the lack of good enough executives, managers or decision makers that can be able to cope with the complexity of being a leader in an university. There are several variables to consider when developing a strategy that pursue the achievement of SCA in HEI: academic quality, better professors, good relationship with industry, enrolment of students, research and development, satisfaction of students, position among competitors, new technologies in education, good facilities, good services, the best educative technology, finances, among others.

Top-level managers and executives in HEI are also required for good and effective leadership competences. In addition, complexity in decision-making (from now on DM) process that leaders confront in the day to day operation should be considered. 
Leading academics (professors) and being assertive in decision-making is not an easy task, and it is in charge mostly of department directors. Those directors should be proficient in academic world in order to have academic credibility (Spendlove, 2007) and at the same time they must have proficiency managing and encouraging people to get the results. For those reasons, there are scholars who even attempt to affirm that strategic management in universities is much more difficult than in industry (Boldt, 1991) referred in (Spendlove, 2007). Theory behind individual decision making in complex and ambiguous sceneries and how it can affect the performance of the firm should be studied.

As seen above, strategy in Higher Education Institutions is dynamic and it depends largely on accurate individual strategic decision-making and effective leadership from medium and high-level managers and executives. Thus, in order to understand how medium and top-level directors in HEI behave regarding risk in decisionmaking, the way they balance exploration and exploitation (ambidexterity) and the way both, ambidexterity and DM, affect the performance in their organizations, it was decided to address the general question and the objectives of this dissertation with a qualitative phenomenological exploratory approach.

This dissertation is composed of 6 Chapters, the Tables and Figures sections and the references section. First chapter is about the context of Higher Education Institutes, and especially about private Universities in México to which the Tecnológico de Monterrey University belong. Also the problem definition, the research question, the objectives, and finally the justification and the limitations of the study belong to this chapter. In the second chapter, a deep literature review is divided in 4 sections. The first section addresses the literature review concerned to basic concepts of strategic management; different approaches and methodologies of strategic management are briefly mentioned, also the most representative researchers in the field are referred Porter, Mintzberg, Drucker, March, Ambrosini, Barney, Eisenhardt, Grant, Wernerfelt, Teece, among others. This section provides the basis to understand the development of internal resources approaches Resourced-Based View (RBV) and Dynamic 
Capabilities (DC), which are going to be referred in this dissertation. The second section of first chapter provides the literature review concerning to Resource-Based View, Dynamic Capabilities of the firm and the concept and implications of Ambidexterity. The approach of this section provides the necessary theory for understanding not only the development of those theories, but even more important, the section explains in detail how strategy is based on those theories and how sustained competitive advantage can be obtained. The third chapter deeply addresses the topic of individual decision-making. The aim of this chapter is to show how individual decision-making is made in complex and ambiguous sceneries and how it can represent a VRIN resource, and an enabler of dynamic capabilities. Some cases are exposed, in parallel with main implications of DM in ambiguous and complex contexts. The fourth and last section of this chapter presents the main literature review concerned to Strategic Management in HEI's. The topic is broad, and therefore it was necessary to narrow the scope and select the most important themes related with the dissertation. In that sense, the selected themes related to strategic management in HEI's were: competitive intelligence and positioning, organizational structures and the trap of copying incorrect models of strategy and decision-making and leadership in HEI.

After a deep literature review, the third chapter presents the Methodology used in this dissertation. This chapter is composed by 5 sections which explains the selected approach, the research strategy, the sample, the unit of analysis, the selected campuses and the detailed procedure that was used for addressing the research question; at the end of Chapter 3 comments about validity and reliability are added. Chapter 4 presents a detailed analysis of the results found in the three themes of the research: strategy, risk and uncertainty in decision-making and ambidexterity. Chapter 5 presents the found results for each objective and also for the research question; emergent topics are also addressed. Finally, in Chapter 6, the conclusions, limitations and future research are provided. 


\section{CHAPTER 1. CONTEXT, PROBLEM DEFINITION, RESEARCH QUESTION, OBJECTIVES, JUSTIFICATION, and LIMITATIONS.}

\subsection{CONTEXT.}

This dissertation is written in an age where big and fast changes and challenges are not rare for almost any industry, neither for a classic one like education where it seems that things could keep constant and unchanged ever. In this section the current context of HEI is going to be described, especially those situations that are related with the dissertation research, and that influenced the definition of the question and objectives of research

Academic leaders are facing enormous internal and external pressures and challenges, challenges quite different from those that they were used to deal, challenges that will demand special abilities and behavior in the way they lead their teams and the way they "do strategic management". In that context, Marginson (2006) referred in Khefacha and Belkacem (2009) claims that higher education is confronted with a major situation of change due to the globalization of the economy and the appearance of new forms of management. To the above challenges, I will certainly add other aspects that interfere the dynamic of today's universities; the increasingly low cost of information, which allow all person to have access to excellent courses and information that used to be taught just in universities, and the fact that industry is demanding a complete new profile of graduated students, those who not only demonstrate knowledge but a complete set of attitudes and abilities. According to Khefacha and Belkacem (2009, p. 53) "this context leads higher education to play a foreground role in the economic scene. It represents henceforth a strategic sector and a source of value, on which the long-term future of the nations

depends. As a result, university objectives, its system of organization and governance, its financial bases, its processes of work and its role in the society are all facing a real challenge". 
Strategic decision-makers and leaders in the context of decision-making, very accurately points out "the academic institutions are characterized by multitude levels of decision mingling consensus, negotiation, bureaucratization and hazard. As a consequence, the decision-makers are forced to take the appropriate decisions in order to respond to the expectations of a plurality of stakeholders constituting the university life" (Khefacha \& Belkacem, 2009, p. 55). Since HEI are confronting a very complex sceneries because the complexity of the organizations, in strategic management of those institutions, it is crucial the right choice of those leaders that are going to be in charge of strategic decisions. Mostly those decision makers are related with positions that affect directly academics and students, for example department deans, career directors, presidents of campus, rectors, among others. Looking for a balance between the academic training and the management skills could be a good idea. Cowburn (2005) states that in strategic management it is often argued that public sector organizations, particularly universities, are good to formulate plans, but that when it comes to put ideas into practice, they frequently fail to achieve objectives. Adding in the same theme, Choban, Choban and Choban (2008) states that, "when strategic planning is applied in educational institutions, the process is often truncated by the absence of clearly defined and reliably documented outcomes".

Tecnológico de Monterrey University, a multi-campus university selected for this study, is located in México. Despite México have great challenges to solve in different areas such as political, environmental, social, and educative, as a country, México will play an important role worldwide speaking, because is considered as an important growing economy that is part of the Emerging And Growth-Leading Economies (EAGLE), which considers the ten emerging and growth leading economies and that "jointly, the group is expected to be responsible for half of all global growth in the next decade - compared with 30 percent for the Group of 7 industrialized nations" (Wassener, 2015). Even more, as González et al., (2015, p. 288) point out, "further, several banks and professional services, including Goldman Sachs and 
PricewaterhouseCoopers Economics, predict that Mexico will become the world's fifth-largest economy by 2050". Meanwhile, multi-campus universities represent an option for offering study opportunities different sites geographically separated, some claim that for being multi-campus it is needed to have two or more campuses (Xie \& Zhang, 2009) referred by (Meng, 2015)) and others claimed that three or more campuses are mandatory (Dhliwayo, 2014), but at the end those campuses must be geographically spaced. Despite we can find some studies which arose the doubt about the multi-campus university model, in some regions, like Africa and China multi-campus universities seem to play an important role in providing education and also in perception of students (Dhlimayo, 2014; Meng, 2015). According to Meng (2015, p. 60) "multi-campus pattern meets not only the external needs of the times, the state and society but also the internal needs of various universities competition".

Regarding to private universities, those had grown in the last years in México, mainly pushed by the demanding of students who are not allowed to enter to public universities; for example, the number of persons that demands the entrance to a university has grown from 250, 0000 in 1970's to more than 1.6 millions in 20032004 (Silas-Casillas, 2005). It is such the importance of private universities that in some countries, such as Brazil, Thailand, South Korea and Japan, more than $60 \%$ of students enrolled in HEI are enrolled private universities (Silas-Casillas, 2005), in México, in 2012, approximately 33\% of enrolled students belong to private universities (Ramos, 2012). In the case of México, and increasing number of private universities happened in the last years; those universities could be classified in three types: high profile, medium profile and low profile (Silas-Casillas, 2005). In general the distinctions among the three levels are the external certifications and academic quality. The problem is that the increase of students enrolled in high profile is about five times lower than the growth in medium and low profile private - about $7 \%$ against 35\% in average - (Silas-Casillas, 2005), and that does not benefit to the country. As we shall see in the next section, Tecnológico de Monterrey is a high level multi-campus private University. 
Knowing the context of private, multi-campus universities in México, thus, what factors should be taken into account when making strategic management in the private universities in Mexico and how it differs from current models? There are several factors to take into account in this strategy, in such way they guarantee the positioning in the market, so "the adoption of marketing practices employed in forprofit organizations by higher education institutions has increased markedly. Consequently, university managers are increasingly focused on marketing techniques, such as targeting and communicating with market segments, that can attract new students and retain existing students" (Rindfleish, 2003, p. 148). Part of the results of this dissertation will address this question, and we are going to analyze how are the strategies of medium and high level directors of 4 different campuses in a high cost private University in México.

\subsubsection{Some Precedents about Tecnológico de Monterrey University}

Research is going to be performed in Tecnológico de Monterrey University (from now on it will be named as Tec). Tec is a private university in Mexico, which is composed of 26 campuses distributed throughout the country of Mexico. Since its foundation in 1943, Tec has been continuously innovating to respond to the educational demands that emerge from social, economic, scientific, labor and technological changes. Our vision is to educate leaders who have an entrepreneurial spirit, a humanistic outlook and are internationally competitive. The main differentiators of the institute are: innovative education model which fosters the development of an entrepreneurial spirit, education with a humanistic outlook, global prestige, and connections with alumni, companies and institutions as well. For this reason, according to QS Latin American University Rankings ${ }^{1}$ 2016, Tecnológico de Monterrey was named as the best ranked University in Mexico, and is placed number seven in Latin America. This ranking, evaluate the 300 best institutions of the continent in seven basic criteria: reputation among academics, reputation among

\footnotetext{
1 The QS University Rankings for Latin America, evaluate the 300 best institutions of the continent in some defined criteria:
} 
employers, number of research teachers that publish articles in indexed journals, number of citations of these articles, number of students per teacher, world wide web presence and proportion of faculty holding a $\mathrm{PhD}$ degree. In the current context, we can mention that Tec is not strange to the fast changing environment, in fact, as university it is always innovating and changing the rules of the game. According to information of the university "we educate agents of change who are willing to be increasingly competitive, for the benefit of all; more willing to be than to have, to serve than to possess. At Tecnológico de Monterrey, we educate, transform and serve the people who live in an increasingly complex, turbulent and challenging world, who will use technologies that have not yet been invented, solve problems that have not yet been detected and lead companies that have not yet been created" (further information about the university can be found in the next citation) (Tecnológico-deMonterrey, 2014).

\subsubsection{Context of Some Multi-campus Universities in México}

As multi-campus HEI, Tecnológico de Monterrey and other multi-campus universities, also faced extra challenges that make them more challenging. According to Timberlake (2004, p. 98), "multi-campus institutions commonly deal with tensions arising from the polarized desire for autonomy at the local level and greater control at the center of the organization. Leaders should establish participatory processes within the institution that provide employees with opportunities to make operational decisions locally as well as participate meaningfully in institutional strategic decision-making processes." In particular, for Tec it has been a big challenge trying to find the optimum point for the 26 campuses that offer different bachelor degrees, in which campuses could be enough autonomous to seize opportunities in the region and at the same time being aligned to the central structure and processes to accomplish the common mission and to maintain a high and uniform standard level of academic quality. 
Now, let's discuss how Tecnológico de Monterrey stand out when compared with other 35 good quality private multi-campus universities. In México there are about 1,000 private Universities (Hernández-Yáñez, 2011), which are composed by different academic level universities (Robio-Oca, 2006). In our case we will focus in those multi-campus private universities that have a good academic level, in this case there were considered as a good academic quality universities those that hold FIMPES ${ }^{2}$ certification (Private Higher Education Mexican Institutions Federation, FIMPES, for its acronym in Spanish). We found about 35 private multi-campus universities, which have this FIMPES certification. We did a search of information about each one in order to show some relevant information such: date of creation, population of students, number of bachelor degrees, number of graduated programs, type of governance, cost of tuition fee, among others. The complete Table containing all the information is very extensive and not all the information provide relevant information for this study; thus, a summary of those 35 private universities was done, extracting some data that are useful for comparison purposes (see Table 1). Comparing some average numbers in Table 1, Tecnológico de Monterrey is by far the one with largest in population - almost fourth times the average of population - and also the most expensive one almost fourth times the average of population that is shown in Table 1 -, and that is why we referring this University as a high cost one. But the most important aspect is that according to QS World University Rankings ${ }^{3}$, Tecnológico de Monterrey is by far the best private Ranked University in México. Table 2 shows that just 11 universities are ranked by QS Latin American University Rankings, being Tecnológico de Monterrey the best occupying the $7^{\text {th }}$ place; while in the QS World University Rankings just 3 private multi-campus Universities are ranked among the best 700 and Tecnológico de Monterrey stands out even more occupying the place $206^{\text {th }}$, and being the only one University in México that is among the best 600 Universities worldwide according to the mentioned ranking.

\footnotetext{
2 FIMPES is a group of private Mexican institutions, which aims to improve communication and collaboration of these with each other and with other educational institutions in the country, respecting the particulars of each purpose, so that its members can better fulfill the responsibility to serve the nation.

3 QS World University Rankings evaluate the 900 best institutions worldwide according to some defined criteria: http://www.topuniversities.com/university-rankings/world-university-rankings/2016
} 
In most of cases, private universities confront more challenging conditions for maintaining good finances than public universities, because in private universities there is not government fund (this is the case in Mexico private universities), and the stream of revenues should come from tuition and other funds (Silas-Casillas, 2005). Context of Tecnológico de Monterrey is a more special case because most of the revenues come from tuition (more than $90 \%$ of the total revenue) setting that in a very unique and interesting situation where the student's enrollment is critical for surviving. It is such context; differentiation will be important for getting new students every year. Specific strategies are clue for attracting new students, for example segment profiling for deciding the best geographic zone for recruiting alumni, strategic differentiation, among others would be different approaches for convincing students to enroll in different universities.

\subsubsection{Selected Campuses for the Research}

In order to understand why the selection of 4 campuses, it is important to understand how the 26 campuses that compose the Tecnológico de Monterrey are classified. There are 4 different categories, according mainly to the population of the campus; we can see in Table 3 the distribution from the 26 campuses. In categories A and $B$, are located those campus that are known as campuses that could offer a wide range of careers, because they offer at least 7 complete careers and have a population of Bachelor students above 800 approximately. Category B campuses are those whose conditions are just above the necessary to be considered campuses that could offer a wide amount of careers; they have bachelor degree students population around 800 and 1000 students. On the other side, Category C campus are those with less than 800 students enrolled in Bachelor degree and which in the future are predicted to stop offering complete careers, in order to offer future bachelor students just the possibility to initiate the career from cero up to the fourth semester. Finally Category C campuses are those that will offer just high school level and one year of 
bachelor degree. Those students that will enroll in $\mathrm{C}$ and $\mathrm{D}$ campuses will have eventually had to move to A or B categories campus in order to finish their careers.

The most challenged situation belongs to Category B campuses, because they are always fighting for maintaining them in this Category; they are near the limit of minimum population of 800 bachelor students. If they fall in one or two years of bad performance they could go down to Category $\mathrm{C}$, which means stop offering complete careers, and then reducing employees and reputation of the campus. On the other side, it is almost impossible for category B campuses to rise to Category A, because the population of the cities and the states they belong is much lower by far - we are talking about millions of habitants of difference - than the Category A campuses.

For the above reason, the campuses that could be pushed to deploy the most difficult strategy are the Category B campuses. At the beginning it was my idea to work with four from the five campuses that belongs to Category B (Campus Sonora Norte was not an option because I belong to that campus, and could not be part of the study). Nevertheless, after talking with two Rectors and stakeholders in some campuses about my research and the valuable that it could be for the institution, they strongly recommend me to select one Category A, in this case campus Querétaro. They suggested that because this was the only campus in Category A that had a similar organizational chart and type of directors in the Division of Professional (this is the division where all the different careers belongs) to a Category B and thus, much more reduced that Category A campuses, which had a bigger chart structures with one more level of directors in the Division of Professional. That way, they considered that could be interested that Querétaro belongs to the sample of study. After that discussion, I proceeded to select 4 campuses for the study: three from Category B (San Luis Potosí, León and Chihuahua) and one from Category A (Querétaro).

The aim of the research was to interview high and medium level directors. An important characteristics of those directors, is that they were in charge of the strategy of their campus or divisions and that they have directors on their charge; 
this way, I could assure that they have a team composed by decision makers for deploying their strategies, and who could have relevant information and knowledge for the explorative phenomenological research (Creswell J. W., 2003). At the selected campuses, the Campus Director - the person in charge of all campus - and the two Division of Professional Directors - the persons in charge of Bachelor Careers at the campus - meet these requirements. In Figure 1, a synthetized chart is shown, just to understand the way different directors report to each other. In that Figure, it can be seen that division directors reports directly to the Campus Director; and that Division Directors are responsible for some Departments Directors (those directors in charge of selection and development of professors) and some Career Directors (those directors in charge of leading the students during their career) depending on the size of campus.

Thus, a total of 12 medium to high level directors are already selected for the indepth interviews of this study. As we will see further, all of the directors were treated anonymously, in such way that, their name and reference to the campus never appeared.

\subsection{PROBLEM DEFINITION.}

Problem definition is derived from the context of HEI (specifically private, high level, and multi-campus) and strategic management. Planning and execution are in charge of the different levels of directors existing in the University, such as: deans of academic departments or department heads, academic directors, bachelor program directors, provosts, supervisors, managers, etc. Those directors have different kind of studies and experience depending on the University and its necessities. What is unanimous is that they must perform in a very efficient way for achieving goals and KPI (Key Performance Indicators) due to the increasing complexity of the environment (Tavernier, 2005). 
In Tecnológico de Monterrey, Campus directors, Division Directors and hence departments and careers directors, are asking to accomplish more that 80 KPI (Key Performance Indicators), related to academic quality, research, finances, enrolment of new students, population per career, graduates' employment, minimum teacher credentials, among others. Maybe it is an indicators oriented behavior. All of those indicators depend on the way of directors define the strategy, the way they manage risk, uncertainty and gut in decision-making and also the way they manage ambidexterity, how exploitative (short term) or how explorative (long term oriented) they are.

So the problem that arises is how those high and medium directors in the selected campuses define their strategy, the play of gut in their decision-making process, how they deal with uncertainty and risk in DM and what is the role that plays the type of ambidexterity and the degree of ambidexterity that they deploys. It is needed to understand deeply how those variables and factors are or not interrelated. In the process of understanding how those variables affect, it will be also important to elucidate the characteristics that are inherent to those directors that present certain positive specific behaviors regarding to the topics of the research that favors the strategy and the deployment of dynamic capabilities from those who do not. Also in this process it can be understood why some directors seems to excels in the deployment of dynamic capabilities in their "enterprises" (considering their universities as an enterprise). That is the problem definition.

\subsection{RESEARCH QUESTION.}

Defining the question for this dissertation has been a challenge because around the complexity of the context of Higher Education Institutions, I was pushed to find in which specific areas of strategic management the dissertation could provide valuable information. After analyzing literature review and the experience in this context, I found relevance in risk and uncertainty in decision-making, ambidexterity and the 
definition of strategic management. Taking into account the above, the main question of the dissertation is:

How high level directors behave regarding ambidexterity and risk in strategic decisionmaking and how are they related to performance in 4 campuses of a high cost multicampus private university in Mexico?

\subsection{OBJECTIVES.}

\subsubsection{General Objective}

To understand how high-level directors manage risk and uncertainty in strategic decision-making (DM), how they perform ambidexterity and how the both, DM and ambidexterity, affect the performance.

\subsubsection{Specific Objectives}

a) To understand how high level directors behave regarding to risk and uncertainty in the execution of strategic DM.

b) To understand how directors achieve Ambidexterity and what are the most relevant factors that promote of inhibit it.

c) To understand whether Ambidexterity and risk, uncertain and gut in DM is related with the performance and how it is affected.

d) To understand how directors perceived their own strategy and how it is related to University strategy

e) To understand whether ambidexterity and/or risk and uncertainty in strategic DM can influence dynamic capabilities (and how it affects the organization). 


\subsection{JUSTIFICATION.}

The relevance of México as country, the private universities and the challenges of multi-campus HEI, was explained in the beginning of this Chapter, in Section 1.1. In this section, justification of the study will be mention in order to strength the importance of it. In order to avoid repeating information that will be provided in further sections, just the most relevant points related with this dissertation's justification will be mentioned.

Traditional approaches to strategy where managers, and in general decision-makers, where more concerned for elucidate where to go (strategic planning for short or long period) and how to do (executing the strategy) could be obsolete in most of the industries for achieving a sustained competitive advantage. According to Eisenhardt (1999) "the ability to make fast, widely supported, and high-quality strategic decisions on a frequent basis is the cornerstone of effective strategy. To use the language of contemporary strategy thinking, strategic decision making is the fundamental dynamic capability in excellent firms". We could keep mention more and more citations about why effective directors, executives and managers are required by universities in order to reach the goals and maintain the rhythm of changing environment and conditions in the market.

The way director's cope with short-term strategies and long-term strategies is also an important topic for HEI managers and directors. One of the concerns in today decision-makers leaders is how to effectively reach performance of the university, while maintaining academic credibility with the professors and academics staff. Also, the way they create resources and maintain the day to day operation and the way they maintain the long vision, in order to create differentiators that allows the organization to survive during the time (Barrett, 2010; Shulze, 2008). Maybe an optimal combination of academic and managerial skills is needed, maybe a quite different effective leadership and management of universities is needed for this 
turbulent age. Just to give an example, a typical Department Director in Tecnológico de Monterrey university teach 4 bachelor degree courses per year, he is responsible of the strategy for achieving a good level in up to 40 indicators and, in addition, he is in charge of about 40 professors, then effective leadership combined with effective management are essential for succeed. According to Spendlove (2007, p. 407) "university leadership is fundamentally different from leadership in other contexts, and demands additional competencies". More research about this topic is required, "the need for a more proactive approach to identifying leadership competencies and developing leadership throughout universities".

When exploring literature review about topics related with strategic management in HEI, you can find several theory and cases of decision-making, leadership, strategic planning, strategic execution, the required profile of academic directors, and many of them are going to be mentioned in the Section 2.4 of this dissertation. Nevertheless, there is a gap in literature review when trying to find a relationship among risk and uncertainty in DM, ambidexterity and the deployment of dynamic capabilities in HEI, more over, in private multi-campus HEI. And it is precisely in this gap that the research of this thesis is proposed. The main particular characteristics of this dissertation, which provides a good frame for the justification, are:

- The research will address the above mentioned gap providing information about how directors and managers in HEI could provide the basis for understanding the deployment of dynamic capabilities; it means how they renew the resources of their universities, in order to provide sustainable competitive advantage.

- Exploratory qualitative research will be used as the research strategy, opening the possibilities for emerging themes and setting the base for future research.

- The research will be executed in a high cost multi-campus university in Mexico. As a private university, more than $96 \%$ of the incomes are got through the tuition fee of students, and then excellent actions of student's recruitment will surely 
come up. Being a multi-campus university is also important because the research will provide information that could be useful for this kind of universities that, according to Timberlake (2004), will become more common as they could gain efficiency because the use of new technology. It is also expected, that this research could provide bases for the use of the results in other HEI.

With all the context above mentioned it is very clear that decision-making and the ambidexterity in HEI directors is important to address the complexity of the environment and assure a sustained competitive advantage. This dissertation could provide relevant information for strategic management literature, precisely in topics related with, Dynamic Capabilities, Decision Making and Ambidexterity, and specifically for HEI.

\subsection{SCOPE.}

This research has been taken place in 4 campuses of a Mexican private university. The sample and the number of selected campuses meet with the standards of qualitative research (Creswell J. W., 2003), as described above in Section 1.1.3.

The fact that the scope is limited to this context does not mean that will apply just within the selected university. Results and conclusions could be useful in other context within Higher Education Institutions.

\subsection{LIMITATIONS.}

There are some limitations on this study. The sample of 12 directors in 4 campuses within a multi-campus private University in México represents a limitation because it is questionable the possibility of generalization; being a qualitative study their goal rather than generalization is deepening. However, we can recognize that working with one university, even tough there were 4 campuses, it is limited to an objective of 
deepening, and the fact that we cannot compare results with other private universities in the country or even between private and public universities.

Another limitation is the temporal frame in which this study was realized because it happens in parallel to the implementation of a quite different and radical strategy 4 , and that specific situation could create a different mindset and scenery for the interviewed behavior and answers. Also the selected directors represent another limitation, because "the participants in the study need to be carefully chosen to be individuals who have all the experienced the phenomenon in question, so the researcher, in the end, can forge a common understanding" (Creswell J. W., 2013, p. 83).

\subsection{SUMMARY.}

In this Chapter we introduced important elements that set the basis for the research. First we talk about the context of México as an emergent country, and the multicampus private universities. After that, the context of the selected university was briefly described, and also the sample selection of campus and directors for the interviews. The problem definition, the scope and the limitations of the research were also explained.

\footnotetext{
4 From the 2014 Annual Presentation of Salvador Alva, President of Tecnológico de Monterrey, happened in May, 2014. When he referred to a disruptive strategy at the University.
} 


\section{CHAPTER 2. LITERATURE REVIEW.}

\subsection{THE IMPORTANCE OF STRATEGIC MANAGEMENT: BASIC CONCEPTS ABOUT STRATEGY AND THE PATH TO RBV AND DC}

The term strategic management is a concept that appeared in the mid -'60s, it incorporates two terms (action and methodology) that merge to constitute one action through a methodology, using various tools. This term has been widely used through years, having important changes due to several factors most of them related to the environment and the way it has changed through time.

\subsubsection{Basic Concepts and Importance of Strategy}

We start with the basics; on the one hand we have the history of management, while on the other hand we have the answer to the questions: what is strategy? And what does strategy mean to organizations?

As we look back in history we find that the Industrial Age was the cradle to many renowned personalities in the management field. We have Henry Ford and his description of efficient tasks to reduce waste, we also have Frank and Lillian Gilbreth, Henry Fayol, and Gantt, all of them contributors to the conceptualization of planning tools that are still used nowadays, such as Gantt charts, Maslow's pyramid, Theory X and Theory $Y$ for employee motivation, among others. We can continue mentioning an endless list of authors up to Peter Drucker, who may be the most influential management writer of the modern era. There is a long list of names, events, and debates on whether if management is science, technology, or art. In general terms, we can reduce it to two extremes: Planning and Control, and in the middle we can locate Organization and Management (execution). These are four steps of the management process that will tell us how to use resources in an organization or task in order to achieve objectives in an efficient, responsible and sustainable way that allows us to create shared value (Porter \& Lawrence, 2011). 
Strategy complements the term management, in that way that in an organization, strategy consists of those actions planned by the governing board of an organization that defines the way in which the latter operates and conducts its operations to achieve its objectives (Porter \& Lawrence, 2011) and (Thompson, Peteraf, \& Gamble, 2010). Therefore, designing a strategy implies managerial work looking to answer the "how" in an organization:

- How to manage growth efforts in the enterprise?

- How to build a loyal customer base and leave rivals out of competition?

- How each functional part of the business (research \& development, suppliers in the value chain, production, sales and marketing, logistics, finances and human resources) should be operating?

Strategy comes from the Greek word "strategos" who's meaning is "chief of an army", making reference to a chief in charge of his team which he leads with a specific purpose. In the Theory of Game, strategy is a complete plan, which specifies the options to take to deal any possible circumstance that the firm may found.

It is important to point out that strategy itself denotes evolution, we can see that positioning, one of the most important features of strategy, is considered today as too static in a dynamic business environment (Porter, 1996). According to Mintzberg, strategy is a plan that defines a rational path that should be taking to an action, a guide to face a situation, but a plan that needs action and execution (Mintzberg, 1994) and (Mintzberg, 1988). For Drucker (1954), strategy is a "decisive action". Mintzberg does not agree about strategic planning and management being the same thing or being always connecter, since he considers a plan is something static, while a strategy results in dynamism that contains the word action; "strategic planning isn't strategic thinking; one is analysis, and the other is synthesis" (Mintzberg, 1994, p. 107). 
Drucker (1954) also introduced the concept of management-by-objectives (MBO) nearly 50 years ago. But Drucker's excellent concept was implemented poorly, leading MBO in most organizations to focus on a myriad of local measures and initiatives not linked to high-level organizational objectives or even coordinated with each other. The planning and execution duet was not well understood and was not well set in operation. The Balanced Scorecard represents a good tool that enables personal objective setting to be integrated across the organization and linked to high-level strategic objectives.

Although it seems that strategy is something easily understandable nowadays for companies they may confuse strategy with operational efficiency. Operational efficiency gives a current picture of good management of resources, while strategy goes further, planning and anticipating. It is a task that every manager should know how to identify; Porter shows us those significant differences in Table 4 (Porter, 1996).

When comparing operational efficiency with competitive strategy, Porter highlights two important characteristics; on one side, we know that strategy relies on unique activities that add value to customer and therefore leads us to what Porter's (1996) coined as "strategic position" (that an organization has a strategic position over another in terms of variety, satisfaction of needs or, where appropriate, based on the customer's access to the product or service offered). On the other hand, Porter also mentions "disjunctive strategies", that means if you want to achieve a strategic position you can't have them all, you must be selective and selection means without a doubt means a disjunctive. Porter talks about three main differentiations or generic strategies: lower cost, differentiated or focus (Porter, 1980).

Along to what Porter states, Mintzberg (1987) proposes other concept for strategy that comes up with Mintzberg 5P's - plan, ploy, pattern, position, and perspective Mintzberg suggests the need of an eclectic definition of strategy, since it can't be 
excluded from each of the 5P's. It requires strategy to be a synergy of these five concepts in order to understand it and most important apply it.

Summing up, Mintzberg (1987) defines strategy as a plan, strategy deals with how leaders try to establish direction for organizations, to set predetermined courses of action. Strategy as a plan also raises the fundamental issue of recognition - how intentions are conceived in the human brain in the first place, indeed, what intentions really mean.

As a ploy, strategy takes us into the realm of direct competition, where threats and feints and various maneuvers are employed to gain advantage. This places the process of strategy formation in its most dynamic setting, with moves provoking countermoves and so on. Yet ironically, strategy itself is a concept rooted not in change but in stability - in set plans and established patterns. How then to reconcile the dynamic notions of strategy as ploy with the static ones of strategy as pattern and other forms of planning?

As pattern, strategy focuses on action, reminding us that the concept is an empty one if does not take behavior into account, if it is not executed. Strategy as a pattern also introduces another important phenomenon in organizations, that of convergence, the achievement of consistency in behavior. How is this consistency composed of, where does it come from? We realize strategy is an important means of conceiving and describing the direction actually pursued by organizations, and when considered alongside strategy as plan, encourages us to consider the notion that strategies can emerge as well as be deliberately imposed. It means that aligning strategy is crucial for achieving objectives (Cohen, 2008), and that execution could be always more important than ideas.

As position, strategy encourages us to look at organizations in context, specifically in their competitive environments - how they find their position and protect them in order to meet competition, avoid it, or subvert it. This enables us to think of 
organizations in ecological terms, as organisms in niches that struggle for survival in a world of hostility and uncertainty as well as symbiosis. How many choices do organizations have, how much room for maneuver? Intra-organizational ecology of strategy approach could be very important in this context, the way all of the employers in the chart align efforts to come up with new SCA or maintaining operating the current strategy (Burgelman, 1991).

And finally as perspective, strategy raises intriguing questions about intention and behavior in a collective context. If focuses our attention on the reflections and actions of the collectivity in order to share norms and values. Ultimately, it is this view of strategy that offers us the best hope of coming to grips with the most fascinating issue of all, that of the "organizational mind". We could relate this topic with the concept of distribute decision-making where people at all levels are part of the decision-making process aligned with the interest of the firm (Worley, Hitchin, \& Ross, 1996).

Therefore, strategy is not just about how to deal with an enemy or a set of competitors or a market, as it is treated in so much of the literature and its popular usage. It also draws us into some of the most fundamental in its popular usage. It also draws us into some of the most fundamental issues about organizations as instruments for collective perception and action.

Once we understand the conceptual framework towards strategic management and that every author agrees on its importance, the question is why so many companies do not own a strategy? Or maybe we could change the question why so many companies think that have a strategy but they do not? Maybe it is because not all companies have those "genius" that Steiner (1979) referred in Mintzberg (1994) when claiming that "if organization is managed by intuitive geniuses there is no need for formal strategic planning. But how many organizations are so blessed? And, if they are, how many times are intuitively correct about their judgments?" 
When companies understand the difference between planning and strategic thinking, they can get back to what the strategy-making process should be: capturing what the manager learns from all sources (both of the soft insights from his personal experiences and the experiences from others throughout the organization and the hard data from market research) and then synthesizing that learning into a vision of the direction that the business should pursue (Mintzberg, 1994). Then, pathdependence and also the way executives make decision-making are important for strategy. In this context, leaders and managers with distinctive and complementary abilities are needed (or finding the "genius"), managers who are able to plan and organize - right handed planners - and creative, risky and well-experienced managers who are able to execute - left handed planners (Mintzberg, 1994).

For instance, for Porter the essence of strategy consists in finding competitive advantage, "the job of the strategist is to understand and cope competition" (Porter M. E., 2008). As long as the strategy to follow is correctly identified you'll be competitive and therefore an organization can become highly profitable. "The strongest competitive force or forces determine the profitability of an industry and become the most important to strategy formulation" (Porter, 2008, p. 3).

One of the main reasons where strategy relies its importance is that it not only determines which activities the company must focus but also how good executive recognize the best tasks that should be kept and evolve to reflect changes in the competitive landscape (Kaplan \& Norton, 2000). On the other hand, responding to the question why strategy is important? Kaplan and Norton (2004) support that every company's strategy describes its intentions to create value for their shareholders, customers and citizens. This way even though strategy is internally formulated its impact or execution will be reflected on the firm's external environment. Then, strategy will be the compass for every company's decision (Porter, 1996).

Finally, we can summarize that strategy is important because of the following reasons (Mintzberg, 1987): 
(1) Sets Direction

(2) Focuses Effort

(3) Defines the Organization

(4) Provides Consistency

\subsubsection{Considerations about Strategic Management Process and Environment}

There are several approaches for strategic management process; we briefly address some of the most important. Strategic programming involves three steps: codification, elaboration, and conversion of strategies (Mintzberg, 1994). Codification means clarifying and expressing the strategies in terms that are sufficiently clear to replicate them formally operational, so that their consequences can be worked out in detail. This requires a good deal of interpretation and careful attention to what might be lost in articulation: nuance, subtlety, and qualification. Elaboration means breaking down the codified strategies into sub strategies and ad hoc programs as well as overall action plans specifying what must be done to realize each strategy. And conversion means considering the effects of the on the organization's operations (Mintzberg, 1994, p. 112).

With a different approach, Porter (2008) indicates that the strategic management process is linked to the 5 competitive forces model; understanding the forces that shape industry competition is the starting point for developing strategy. Every company should already know what the average profitability of its industry is and how that has been changing over time. These forcers reveal the most significant aspects of the competitive environment. They also provide a baseline for sizing up a company's strengths and weaknesses. 
In addition, a very important aspect in the process of strategic management is the level of employee involvement and their consequences of their actions. Daniels and Bailey (1999) studies have demonstrated that Participative Decision Making (PDM) can be beneficial to worker mental health and job satisfaction affecting job satisfaction in one of two ways. First, as a direct effect upon job satisfaction that reflects an intrinsic biological drive to influence the environment or feelings of mastery and competence associated with exerting influence successfully over the environment. Second, because PDM allows individuals to manipulate the environment to reduce sources of stress.

Environment is also an important issue for companies to consider. Same scholars, Daniels and Bailey (1999) includes 6 different dimensions:

(1) The command dimension

(2) The planning dimension

(3) The incremental dimension

(4) The cultural dimension

(5) The political dimension

(6) Enforced choice

The command dimension. Strategy is focused in a central figure, in such way that the job satisfaction of others individuals will be reduced.

The planning dimension. Well-defined goals and objectives (eliminate ambiguity) that are shared to all organization and periodically evaluated.

The incremental dimension. Strategy development is iterative, they can be changed, incremented, reduced or analyzed several times in order to retain successful ones. 
The cultural dimension. Strategy elaboration depends on managerial experience, expectations, and beliefs.

The political dimension. Taken into account stakeholders (groups of power).

\subsubsection{Considerations about Strategic Formulation and Execution}

Formulation and implementation refers to how strategy is defined by an organization for an instance and the process that every leader deals when taking necessary actions to follow the strategy to what we have called implementation.

Mintzberg mentions that strategy formulation in every organization is only possible to achieve as long as it is clear: a) the concept of strategy, b) the very different views of how it can be formed (without necessarily being formulated) in an organization, and (c) to associate this view especially, but not exclusively, with the configuration we are calling adhocracy (Mintzberg \& McHugh, 1985).

The concept of adhocracy is an expression first used by Alvin Toffler and later made popular by Robert Waterman (1990) in his book "Adhocracy - The Power to Change"; the term corresponds to the opposite of bureaucracy: while bureaucracy claims for rigidity of routines, adhocracy wants to simplifying processes and allows fast organizational adaptation to particular situations. The essence of adhocracy, in contrast to bureaucracies that seek to control their environments to support standardized systems, is a rapid and continuous responsiveness to the environment, with minimal organizational momentum.

About planning versus execution, Mintzberg (1988, p. 72) claims that "the crafting image better captures the process by which effective strategies come to be. The planning image, long popular in the literature, distorts these processes and thereby misguides organizations that embrace it unreservedly". Formulation and 
implementation merge into a fluid process of learning through which creative strategies evolve. Craft evokes traditional skill, dedication, and perfection on action. They should be not separated, planning without execution is meaningless.

Porter tells us that in the formulation of a strategy it is important to understand the forces that affect the competitive environment in an industry, and at the same time, it is necessary to identify the factors that affect it, this way, the company's strengths and weaknesses over its competitors, customers, etc. can be easily visualized. That is why a strategist can devise a plan of action that may include "(1) positioning the company so that its capabilities provide the best defense against the competitive force; and/or (2) influencing the balance of the forces through strategic moves, thereby improving the company's position; and/or (3) anticipating shifts in the factors underlying the forces and responding to them, with the hope of exploiting change by choosing a strategy appropriate for the new competitive balance before opponents recognize it " (Porter, 1979, p. 143).

As already seen, the challenge of strategic management lies not only in the strategy formulation but also in the correct implementation, since both of them determine the success or failure of Strategic Management as such. Kaplan and Norton (Kaplan \& Norton, 2001) maintain that success in the implementation is concentrated on two words: alignment and focus (strategy-focused organization). Although the specifics of each company can lead to different roads when speaking of alignment and focus, according to (Kaplan \& Norton, 2001) referred in (Knapp, 2001), the Principles of a Strategy-Focused Organization are: Translate the Strategy to Operational Terms, Align the Organization to the Strategy, Make Strategy Everyone's Everyday Job, Make Strategy a Continual Process and Mobilize Leadership for Change.

With this briefly travel around the basic concepts of strategic management, we are ready to move on to the next chapters. In the next chapters, extensive literature review about resource-based view, dynamic capabilities, decision-making and leadership in HEI will be provided. Basic concepts about implications in strategic 
concept, strategy process, strategy formulation and strategy implementation were key for understanding more elaborated concepts in the rest of this document.

As we shall see, a resource-based view of the firm and dynamic capabilities are treated as critical fields of strategic management in creation of competitive advantages and as motors to new important fields knowledge-based view or ambidexterity. Just to give an idea of the relevance of RBC, according to Wernerfelt (1984) referred in Hitt, Gimeno and Hoskisson (1998, p. 12), "perhaps the most important recent development in the field has been the acceptance, integration and application of the resource-based view of the firm". Focusing on internal resources and capabilities, resource-based view and dynamic capabilities strategies can provide a more long-lasting competitive advantage than the traditional product/market approach; this does not mean that external environment and proposals of industrial organization from Porter must be taken away, but complemented. 


\subsection{RESOURCE-BASED VIEW, DYNAMIC CAPABILITIES AND AMBIDEXTERITY: ACHIEVING COMPETITIVE ADVANTAGE THROUGH INTERNAL RESOURCES AND COMPETENCES}

There are strategic approaches that have in common the fact that rents flow from privileged product market positions, and make reference mainly to the external environment. This kind of approaches were the dominant paradigms during the late 1970's and 1980's, the best example of those approaches are The Five Competitive Forces by Porter, who claims that firms can create defensible positions in industry according to external forces, which shape the strategy to implement (Porter, 2008). Nevertheless, environment started to change in shorter periods of time, entering in turbulent times, making that those approaches were not sufficient for reacting to a rapidly changing industry. Even more, competitive advantages of companies did not last as much as they used to last, making harder to differentiate among competitors (Eisenhardt \& Martin, 2000). In that context, distinctive approaches began to appear, approaches that were based on internal resources, trying to build sustained competitive advantage (hereafter SCA) through capturing entrepreneurial rents that comes from firm level efficiency advantage. Those approaches, acquired an essential role in strategic management in the last years. The approaches we are talking about are the Resourced-Based view of the firm (hereafter RBV) and Dynamic Capabilities (hereafter DC). To put it in perspective, the RBV can be located between the years of 1990 and 2000, and also dynamic capabilities arises about the same time. Both perspectives are trend topics in strategic management, and also contributed a disruptive change in the way of conceiving the firm.

In this section of literature review, our attention will be focused on the role of internal resources, routines and processes as the bases of sustained competitive advantage into what is now known as the resource-based view of the firm (RBV) and Dynamic Capabilities (DC). First, the relevance of RBV and DC approaches and the main characteristics of those are briefly mentioned. Second, RBV and DC are examined as an important piece to achieve SCA. Later on, we deepen into some 
examples and the manager's importance when using these RBV and DC approaches. Then the concept of Ambidexterity is mentioned, and also the main theoretical frame about the theme that will be needed for this dissertation. Finally, conclusions and personal comments are done. Let's start.

\subsubsection{UNDERSTANDING RESOURCE-BASED VIEW AND DYNAMIC CAPABILITIES}

General annotations about Resourced-Based View

In 1959, Edith Penrose published the Theory of the Growth of the Firm, and made an important change in how firms are seen. This book settled that firms are "flesh and blood" organizations, and not just a point on a cost curve. According to the theory of the growth of the firms, the latter consists of human and non-human resources, under administrative authoritative coordination and communication (Pitelis, 2005). Penrose focused on the internal resources of the organizations where knowledge, added value, innovation and, in general, competitive advantage could be generated. For Penrose, managers play a very special role in the growth of firms. Penrose saw the external environment as an "image" in the minds of managers (Pitelis, 2005).

Although Penrose settled down all the bases about "flesh and blood" firms in the late 50 's, it was until the 80 's that internal resources and capabilities gained more importance, triggered mainly by three factors (Grant, 2010): first, the growth of more unstable environments that required more secure bases for formulating strategy; second, the flourishing idea that competitive advantage rather than industry attractiveness was the main source of profitability; and last but not least, the existence of a world where customer preferences and new technology rates are volatile.

RBV and DC as we shall see, correspond to the strategic creation of sustainable competitive advantage focusing in internal resources and complement the focus on industry structure that Porter has claimed for years (Brahma \& Chakraborty, 2011). 
Differences between those two big areas of approaches to strategy arise with many scholars arguing what are the differences and how they complemented each other. According to Brahma and Chakraborty (2011), superior skills of different forms arise from the way they play in industry, for example trying to maximize the advantages of five forces of Porter, an approach what we call today Industrial Organization. Meanwhile RBV in Brahma and Chakraborty (2011, p. 9), Barney (1986) argued that "if the factor market resources are perfectly competitive, it is not possible for a firm to get economic rent even if it is successful in creating an imperfect product-market because the price paid to such resources will be equal to its value that the resource will create in the product-market. There is an imperfection in the factor-market which is the result of luck or insights of the firm." In that way, "Porter emphasized on the creation of imperfection of a firm's product-market for competitive advantage" (Brahma \& Chakraborty, 2011, p. 11).

RBV strategy focuses on the optimization of the role of resources and capabilities as the principal basis for a sustainable competitive advantage (SCA). The RBV is a theory centered on the nature of firms based on its resources, as opposed to theories such as transaction cost economics, which seeks to explain the reason why firms exist (Lockett, Thompson, \& Morgenstern, 2009). Another way to define the RBV is as a determined collection of assets or resources that are tied "semi-permanently" to the firm (Wernerfelt, 1984) and (Lockett, Thompson, \& Morgenstern, 2009).

The RBV has had a major impact on strategy because the typical product/market orientation is no longer suitable due to the constant and rapid change of the external environment and costumer preferences. It is easy to catch this if we consider that it is more feasible to control internal resources and capabilities to face the real world, than changing the world to adapt to the firms' needs. The RBV of the firm allows us to respond significant questions such as: On which of the firm's resources should diversification be based? Which resources should be developed through diversification? (Wernerfelt, 1984). 
What are resources according to $R B V$ ?

Firm resources include human resources, assets, organizational processes, information and knowledge, among others. In a general sense, resources can be classified as tangible, intangible and human. On the other side, resources can be static or dynamic.

Regarding resources Lockett, Thompson, \& Morgenstern (2009) point out that there are three central elements in the RBV: resource functionality, resource combination, and resource creation and decay. By combining resources, firms are able to add value. In fact, Penrose argues that the "opportunity set is also influenced by the way managers combine resources to produce productive services or capabilities" (Lockett, Thompson, \& Morgenstern, 2009, p. 14). The creation of resources depends on the history of the firm; it can be seen that each firm possesses a bunch of resources whose value is in constant flux. According to Lockett, Thompson and Morgenstern (2009, p. 16), "if resource markets are perfect, the costs of acquiring resources will be approximately equal to the value of those resources once they are used to implement product market strategies. Consequently, if a firm acquires resources, and continues to use them in the same way that they were previously employed, SCA will be difficult to achieve in the absence of resource market imperfections".

Barney (1991) defines the following attributes or characteristics of a resource: (a) it must be valuable, in such way that it is capable to exploit opportunities and beat threats, (b) it must be rare among other resources, in such way that it happens to be quite difficult to find the same resource in competition; (c) it must be imperfectly imitable and (d) it must not have equivalent substitutes, this means that rare and valuable resources can only result in sustained competitive advantage. Due to the initials of each characteristic, these are called VRIN (Valuable, Rare, Imperfectly imitable, and Non-substitutable). Resources must have VRIN attributes, but just having those attributes is not sufficient for achieving competitive advantage, 
nevertheless sustainable competitive advantage. Rare and valuable are two of the main characteristics of RBV, both are equally important for achieving competitive advantage, but a valuable resource depends not only in the intrinsic nature of it, but in the way it is used or the way it is deployed.

As we shall see, the way resource is used or combined will depend in most of situations on the decision making of the managers or administrators in charge. According to Brahma and Chakraborty (2011), from the point of view of VRIN resources, for a firm to have a sustained competitive advantage the resources should be not just rare and valuable, but inimitable and non-substitutable. For example, you can have a specific technology that can give you advantage and introduce new products or new features that may improve the revenues and the position of your firm, but if that technology is easy to copy or emulate, the competitive advantage will last a short period of time and hence not sustainable. Important to mention that resources should be replaced once they lost any of the VRIN characteristics, in order to maintain SCA.

\subsubsection{Toward a Dynamic Capabilities approach from Resource-Based View}

RBV strategy seems to be not enough to support significant and sustained competitive advantage, especially in rapidly changing environments. The problem with RBV is that the view of the firms as a bunch of resources is very static and limited and does not provide explanations on how successful firms endure over time with an increasing competitive environment. For example, firms like IBM, Texas Instruments; Phillips among others seems to stick to RBV approach of accumulating valuable technological assets as VRIN resources (Teece, Pisano, \& Shuen, 1997). Nevertheless, those firms that have sustained good positions seem to demonstrate timely responsiveness and rapid adaptation to environment through internal changes in their structure and resources. It seem that they have mastered the management capability to coordinate and redeploy internal and external resources and competences (Teece, Pisano, \& Shuen, 1997). This ability to achieve new forms of 
competitive advantage through the renovation of based resources and competences belongs to dynamic capabilities approach.

According to Barney (1991), dynamic capabilities follow the theory of RBV of the firm. As a matter of fact, DC can be seen as a complement to RBV approach. According to Teece, Pisano, \& Shuen (1990) when refereeing to RBV in Ambrosini and Bowman (2009, p. 30), "is not only the bundle of resources that matter, but the mechanism by which firms learn and accumulate new skills and capabilities, and the forces that limit the rate and direction of this process". In this way, they propose an interesting definition of dynamic capabilities as follows: "the firm ability to integrate, build, and reconfigure internal and external competences to address rapidly changing environments" (Ambrosini \& Bowman, 2009, p. 30).

There are several definitions of DC in the literature of strategic management. Spending time analyzing them could be worthless, it is just important to emphasize that they refer to resources or routines configurations that are learned or created by managers according to their knowledge and history (path dependence) in order to address rapidly changing environments. It is important to remark that dynamic capabilities are intended processes that arise inside the firm but with the welldefined purpose, it is not about luck or emergent situation.

Notice that RBV and dynamic capabilities refer more to strategy through performance rather than through the market position approach that is posted by Porter in the 80's. They also highlight internal resources and the path dependence of them, instead of focusing on external environment like Porter proposes. But DC are complex by themselves, because they refer to changing internal factors, most of the times resources that are not measurable, in this way it is important to consider more theories as concerned to DC, theories like process of creative destruction and innovation-based competition and the role of firm-specific assets and isolating mechanisms (Ambrosini \& Bowman, 2009). This approach is relevant in a 
Schumpeterian world of innovation-based competition (Teece, Pisano, \& Shuen, 1997).

Understanding the correct use of the term "Dynamic Capabilities"

Dynamic capabilities are not capabilities by themselves neither are they resources. When referring to the term dynamic capabilities, we always must use both words together; otherwise the meaning surely is not going to be the correct. Let's go deeply in the very particular meaning of each word when used in DC.

Let's start with the word "capabilities". The word "capabilities" has a very different meaning when used in dynamic capabilities compared to the classical meaning in other contents. In RBV for example, capabilities are processes or routines that arises from VRIN resources, but they refer to today capabilities as they are static in the way that they refer to today's abilities to be different and hence to have a competitive advantage. On the other hand, in dynamic capabilities, the term "capabilities" emphasizes "the key role of strategic management in appropriately adapting, integrating, and reconfiguring internal and external organizational skills, resources, and functional competences to match the requirements of a changing environment" (Teece, Pisano, \& Shuen, 1997, p. 515).

On the other hand, the word dynamic refers to the capacity to renew competences and resources to have congruence with the changing environment (Teece, Pisano, \& Shuen, 1997) and it is future oriented because it refers to processes that change the based VRIN resources, instead of just using and combining them in different ways. Dynamic refers to the change in environment, but not to the capability of being dynamic. In RBV capabilities refers by which the resources are utilized. It refers to the abilities to adapt and change constantly internal and external resources to address the changing environment (Ambrosini \& Bowman, 2009). 
Path dependence and Heterogeneity

Two main characteristics of the RBV are path-dependency and firm heterogeneity (Lockett, Thompson, \& Morgenstern, 2009). As Lockett (2005, p. 85) mention, Penrose considered the firm as "administrative organizations that are collections of heterogeneous productive resources that have been historically determined".

RBV is path dependent because firm resources are directly related to firms' past activities, this can be determinant on increasing or decreasing growth through time. When talking about heterogeneity, the RBV offers an interpretation of the existence of profits in equilibrium. In other words, any source of competitive advantage is simply a rent conferred by one or more imperfections in the resource market (exogenous variables) that prevent an equitable input allocation among competitors. Resources differ in their impact on the firms' ability to generate profit or differentiation advantages, and hence, performance.

When referring to dynamic capabilities, heterogeneity does not apply, as we shall see later in this paper, mainly because routines and processes that compose DC share commonalities among different firms. Nevertheless, and as we also shall see, it does not mean that homogeneity yields to the lack of a sustained competitive advantage because the most important thing for dynamic capabilities is to generate new heterogeneous and VRIN resources.

Path dependence and position are very important for dynamic capabilities. "The literature characterizes dynamic capabilities as complicated routines that emerge from path-dependent processes" (Eisenhardt \& Martin, 2000, p. 1114). This path dependence provides knowledge learned through years to the firm, but also important for developing DC is the contribution of small loses in the learning process. Repeated practice accelerates and improves the creation of DC. According to Ambrosini and Bowman (2009), not only path dependence is important but also position of the firm; both are internal factors and their importance resides because 
they are concerned with two aspects that play a critical role in the effective deployment of dynamic capabilities: learning and the existing set of resources, as we saw previously. Finally, experience also plays a key role in developing DC; according to Eisenhardt and Martin (2000), there should be an adequate amount of experience, because in some situations experience that comes to fast can overwhelm managers, on the other hand infrequent experience can lead to forgetting what was learned previously.

\subsubsection{RESOURCE BASED VIEW AND DYNAMIC CAPABILITIES AS SOURCES OF PROFIT AND SUSTAINED COMPETITIVE ADVANTAGE}

A resource is anything in a firm that can be thought or considered as a strength or weakness (Wernerfelt, 1984). In general terms, we can identify two major sources of superior profitability: industry attractiveness and competitive advantage; of these, competitive advantage is by far the most important (Grant, 2010). In the last years many researchers have spent time and research in trying to identify which resources provide SCA, to mention some of them that were identified: human resources ((according to Amit \& Schoemaker, 1993) mentioned in Brahma \& Chakraborty (2011, p. 9)), organizational culture (according to Barney, 1986) mentioned in Brahma \& Chakraborty (2011, p. 9), organizational routines (according to Nelson \& Winter, 1982) mentioned in Brahma \& Chakraborty (2011, p. 9), response lags (according to Lippman \& Rumelt, 1982) mentioned in Brahma \& Chakraborty (2011, p. 9), invisible assets which are difficult to imitate (according to Itami, 1987) mentioned in Brahma \& Chakraborty (2011, p. 9), research and development assets.

The relationship between firm resources and competitive advantage is highly correlated. Resources must be heterogeneous and not perfectly mobile in order to get competitive advantage (Barney, 1991). In addition, resources should have specific features that allow us to measure or probe their heterogeneity and immobility levels. VRIN framework of resources sets out the broad necessary conditions for a resource's comparative scarcity to elevate it to strategic significance 
(Lockett A. , 2005). It is clear that any resource that is considered a source of competitive advantage must have the VRIN framework in order to exploit differences and create heterogeneous and immobile scenarios for the firm. When used in the correct way by the firm, the VRIN resources can offer competitive advantage to any firm, due to the ability to provide unique and inimitable internal assets.

RBV approach claims that competitive advantage is found when a firm achieves VRIN resources, but VRIN resources are static in a specific frame of time and in some moment in the future, competition could copy or destroy them, eliminating the competitive advantage. Then, if the objective of a competitive advantage is to generate a differentiation through the time we could wonder if the static approach of RBV could generate real and sustained competitive advantage in a firm? Up to know it seems that VRIN resources are basic for the creation of competitive advantage, but maybe they are not enough to get a sustained one. "A valuable resourced base (and hence capabilities) allows a firm to earn a living in the present" (Ambrosini \& Bowman, 2009, p. 34). Going deeply in literature, it seems that DC is a complement to the static approach of the RBV to get real and sustained competitive advantage. According to Winter (2003) quoted in Ambrosini \& Bowman (2009, p. 32) "dynamic capabilities govern the rate of change of a firm's resources and notably its VRIN resources". But the critical point here is that, a firm with unused VRIN resources is not able to generate competitive advantage; DC allow firms to continually have competitive advantage and help firms to avoid developing core rigidities (Ambrosini \& Bowman, 2009). Then, the goal of dynamic capabilities is to generate a new bundle of VRIN resources.

Let's move further in the last idea. Despite what we have seen, some authors like Ambrosini and Bowman (2009) consider that due to the abstract and intangible characteristics of DC, the effect in firm performance is indirect and not direct like with VRIN resources. Moreover, in agreement with Eisenhardt and Martin (2000), as processes and routines, dynamic capabilities possesses several commonalities among firms and those commonalities imply that DC per se are not likely to be 
sources of competitive advantage, because "firms can gain the same capabilities from many paths, and independent of other firms" (Eisenhardt \& Martin, 2000, p. 1110) and thus they are not unique nor inimitable. Then we can infer that dynamic capabilities are necessary but not sufficient for creating sustained competitive advantage because they could be duplicated across firms. Moreover, depending on how valuable, rare, inimitable are the new-based resources created by specific dynamic capabilities, the performance of the firm could be positively or negatively influenced. Positive changes occur when the based resource that arises from dynamic capabilities provides a better performance in the firm. On the other hand, if the new base resource provided by the DC yields to a poorer performance the result is negative. The final possibility is that DC provides just temporary competitive advantage instead a sustained one, if the new emerged VRIN resources are easy to imitate for the competitors.

As noted from above paragraphs, achieving sustained competitive advantage from resource-based view and dynamic capabilities is not a straightforward process neither an easy one for any firm. Dynamic capabilities do not necessarily lead to sustained competitive advantage; even more they could provide negative results. Then we wonder how could we sum-up in a nutshell the way to get sustained competitive advantage? The focus is on asset structures for which no market exists and in creating continually new VRIN resources in such way than these resources improve the firm's performance; a key step in building competitive advantage "lies with its managerial and organizational processes, shaped by its specific asset position, and the paths available to it" (Teece, Pisano, \& Shuen, 1997, p. 518). As we shall see further, managers play an important role for getting sustained competitive advantage. 


\subsubsection{EXEMPLIFYING APPLIED STRATEGY BASED IN RESOURCE-BASED VIEW AND DYNAMIC CAPABILITIES}

As referring to internal resources, most of the time some commonly unobservable like human resources and other implicit resources such position of the brand and organizational learning (Lockett, Thompson, \& Morgenstern, 2009, p. 16), RBV and DC are still questioned when trying to be measured by common quantitative methods. Nevertheless, there are several examples of papers that treat topics related with achieving SCA through the application of RBV theory of the firm.

Unique capabilities in different industries could be a source of competitive advantage. For example in semiconductors industry, the way they do research and the way they develop technology can create the difference for surviving in such competitive and changing environment; even more, internal decisions, and the way managers in different levels provide the conditions that involved different employers in the strategy could benefit in having a successful strategy (Burgelman, 1991).

In several different industries, research could be the important and unique difference. In pharmaceutical industry, for example, the ability to conduct research and the way this research provide new products can accomplish important dynamic capabilities that separate those firms that survive from those who will not (Henderson \& Cockburn, 1994). Henderson and Cockburn (1994) provide a detailed study of pharmaceutical industry claiming that the ability to integrate knowledge both across the boundary of the firm and across disciplines and product areas within the firm is an important source of strategic advantage. In fact, in those technologydriven industries, according to Dierickx and Cool (1989) refereed in Henderson and Cockburn (1994, p. 3) "a substantial body of theoretical work suggests that idiosyncratic research capabilities are likely to be a particularly important source of strategically significant competence in science and technology driven industries".

Sometimes, intangible resources play a very special role as VRIN resources. It is well known that those resources are quite difficult to measure or observe. A specific case 
is the role of managers, important role that is shaped by the set of decisions that are taken day by day, and that are not measured neither observed one by one. Next section will go deeply in the way managers' behavior constitutes a kind of resource and sometimes a capability that can provide sustained competitive advantage in a firm.

\subsubsection{Role of managers in resourced-based view and dynamic capabilities strategies}

When talking about RBV strategy, resource advantage can be both exogenous and endogenous. According to Lockett, Thompson and Morgenstern (2009), the role of a manager is similar to that of a card player: the player has cards determined exogenously depending on the environment (external factors). Success in the game depends on how the player takes advantage of those cards, and the subsequently acquired cards (endogenous factor).

CEOs and managers in middle and at top levels could be considered as unique resources, that per se have unique path dependences that constitutes VRIN resources and provides SCA. Brahma and Chakraborty (2011, p. 13) referring to Smith, Carson and Alexander (1984); Pfeffer and Davis-Black (1986); and Haleblian and Finkelstein (1993) claims that "past researchers have found that organizational performance is associated with executives performance, top-managers team size, composition and tenure". According to Oliver (1997) cited in Brahma \& Chakraborty (2011, p. 13) "a firm's sustainable advantage depends on its ability to manage the institutional context of its resource decisions which includes internal culture and broader influences from state, society and inter-firm relations". Managers do all these things. It is easy to show that firms like INTEL, APPLE, and FORD installed the culture that CEO brings to the firm, CEO culture and experience depends on path dependence, among other factors. 
Most of the time, managers are in charge of executing the master plan of the firm's corporate or business strategy. As Mintzberg (1988) said, it is about crafting a strategy that requires managers to know about the business and apply their knowledge and experience to create strategic differentiation. The SWOT and marginal analyses are very useful and allow most managers to be capable to react to the internal and external environment, trying to create and develop new actions and strategies that enable firms to survive among competitors. Nevertheless, RBV approach permits managers to gain a better understanding of market imperfections, not just on resources but also on products, to accomplish SCA. Under the resourcedbased view and dynamic capabilities, managers have the possibility to continuously reposition the firm according to internal and externals resources. On the other hand, industrial organization economics consider that the role of managers is responsive to different situations. Thus, in RBV (and we can extend to dynamic capabilities as well), managers have the chance to be more proactive and adaptive to different situations (Lockett, Thompson, \& Morgenstern, 2009).

A manager is responsible for the profitable usage of the available resources. Thus, a manager's perception is an important dimension in the RBV. From another point of view, the fact that a manager's perception affects resource allocation assures that resources have plenty of different usages among different firms, supporting the heterogeneity theory that Barney held (Barney, 1991).

Great managers' challenge in the use of RBV and dynamic capabilities strategy is not only detecting and anticipating future competitors (Burgelman, 1991) but also being thoroughly acquainted with the functionality of resources that are under their control, recombining them in a range of different ways and determining the most profitable usage for these resources depending on the market.

Managers are also crucial for the correct deployment of dynamic capabilities; more over it should be one of the main concerns in their positions. According to Ambrosini and Bowman (2009), managers are one of the internal factors that most influence 
dynamic capabilities, because they always play a key role in the firm's ability to adapt to the environment. To resume the importance of managers and the relationship with path dependence, according to Teece (2007, p. 1346) quoted in Ambrosini and Bowman (2009, p. 41), "dynamic capabilities reside in large measure with the enterprise's top management team" but, because of path dependency these dynamic capabilities "are impacted by the organizational processes, systems, and structures that the enterprise has created to manage its business in the past".

\subsubsection{DEPLOYING DYNAMIC CAPABILITIES}

\subsubsection{Understanding the creation of dynamic capabilities}

Studying and defining the creation of DC is not such and easy task; dynamic capabilities are not well defined and, until now, there is not a well-defined followthe-rules process to create them. About creation of DC, first it is important to mention that there are internal and external situations or factors that inhibits or enable dynamic capabilities (Ambrosini \& Bowman, 2009): the level of dynamism of external environment (where rapidly changing environments provide better conditions than slow ones), the pace of change in an industry and path dependence (like in RBV history matters), managers. According to other authors there could be more specific factors like social capital, leadership, trust, among others (Ambrosini \& Bowman, 2009).

Focusing on market dynamism in general, we can distinguish two well differentiated sceneries: first with moderately dynamic markets, where dynamic capabilities appear in stable industries, being complicated, detailed, and arising from analytical processes that relay on previous knowledge and are linear to produce well known outcomes. In contrast, in very high-velocity markets are different, because the unstable market pushes them to be simple, experiential and quickly giving competitive advantage that could be copied with the time and unpredictable outcomes (Eisenhardt \& Martin, 2000). 


\subsubsection{Examples of dynamic capabilities}

We already saw that there are several factors that could stimulate or discourage the creation of DC. As we will see further, the investigation about creation of DC is not completely clear and there is plenty of room to do. As we pointed out there is little empirical evidence about DC nevertheless, there are well defined DC that have provided competitive advantage to the firms.

As specific processes, dynamic capabilities can integrate, reconfigure, create or release resources. In the case of product development routines, managers integrate resources combining knowledge, experience and skills across different functional teams to provide new and different products that provide a competitive advantage to the firm. In the case of reconfiguration, for example, IDEO manager's routines create new products from knowledge of existing products from many industries and clients (Eisenhardt \& Martin, 2000). Creating resources involve those routines that build new knowledge in the firm, those includes alliance and acquisition to bring new resources from external sources. Acquisitions and alliances are commonly used in biotech industry to achieve superior performance (Eisenhardt \& Martin, 2000). Finally, releasing resources could be also a dynamic capability, primary in rapidly changing environments; it consists in releasing resource combinations that not longer provide competitive advantage.

Research and development constitutes another typical dynamic capabilities, which used in the correct way could provide an effective response to changes in market prices. Related with research and development, product innovation is also a great dynamic capability that provides to the firm the base to renew constantly products and processes. Organizational structure reconfiguration could be also a dynamic capability, letting firms to reconfigure their business units and recombine resources to adapt environmental changes. Besides the above examples, we could find several more dynamic capabilities. For further information we encourage you to check 
(Ambrosini \& Bowman, 2009), (Eisenhardt \& Martin, 2000) and (Teece, Pisano, \& Shuen, 1997) where several examples and references are made deeply. Finally, we must not forget that dynamic capabilities could be similar among different firms but the importance stands in the way that they change the based VRIN resources, to provide a better performance.

Resources sometimes are complex to define or measure. Dynamic capabilities as well, could be difficult to detect. For example, cultural organization in different industries could provide SCA, like the case of Hewlett Packard which culture promotes and strongly pushes for cooperation and teamwork through different division and among different employees.

\subsubsection{Ambidexterity: Context and Concepts}

The term of ambidexterity has been taken importance, just as RBV and DC did, due to the complex environment that enterprises are facing nowadays, and hence the necessity of the firms for addressing and balancing effectively the concerns of the daily operation goals, or short term, with long term goals and projects that could signify the future differentiation of the organizations. As March (1991) referred by (Schulze, Heinemann, \& Abedin, 2008, p. 1) pointed out "in order to ensure long-term survival, organizations are forced to constantly generate new competitive advantages, which underscores the importance of balancing the exploitation of old certainties and the exploration of new possibilities".

The concept of ambidexterity in management could be defined in different ways. For example, Duncan (1976) affirms that ambidexterity refers o the capacity of firms to create dual structures for addressing activities related to the day to day management of the organization (exploitation) and the innovation necessities if the firm (exploration); as we shall see, forty years ago Duncan was referring to a structural ambidexterity. Another way of defining ambidexterity refers to the "firm's ability to continue to exploit its current capabilities as well explore into future opportunities" 
(March, 1991; Levinthal and March, 1993) referred in (Tushman, Smith, Wood, Westermann, \& O'Reilly III, 2004). Regarding to the second definition, March argues that one of the main ways for enterprises for addressing that fast changing world is developing efficient and effective ways of combining exploitative and explorative activities,

Several authors agree that ambidexterity and the pursuing search for a balance of exploration and exploitation at the enterprises is beneficial for the firms (Schulze, Heineman and Abedin, 2008; Raisch \& Birkinshaw, 2008; Gibson and Birkinshaw, 2004). Others affirm that they are certain behaviors when excess in either exploitation of exploration, could bring negative performance. Also, some scholars argued that certain firms due to its nature and environment seems to need a better balance among exploitation and exploration. For example, according to Schulze, Heinemann and Abedin (2008, p. 2) "in a competitive landscape which is characterized by dynamic and highly competitive business environments with short product life cycles, declining costs of technology and an increasing need for production flexibility" it is possible that enterprises must have to address explorative and exploitative activities simultaneously.

\subsubsection{What Exploration and Exploitation means from the Context of Ambidexterity}

We have mentioned the words exploration and exploitation, and the necessity of balancing both as the aim of ambidexterity. But what do exploitation and exploration mean in the context of Ambidexterity?

Exploitation refers to the set of activities that are part of the day-to-dat operation at the enterprises. Exploitation are those activities that enhance the short term operation of the enterprises and it is related to the activities that can bring immediate results needed for the short term survival of the enterprise. Exploitative activities refer to the process of learning through the local search and experience, 
through the "experiential refinement", and the improvement of the best selected and existing routines within the firm (Raisch \& Birkinshaw, 2008). Here it is very important to strengthen the word "existing", because exploitation refers to existing routines while exploration, as we shall see, refers to the search of new ones.

On the other hand, exploration refers to those activities that are aimed to address the future, the long term goals of a firm. Innovation is part of exploration. "Compared to returns from exploitation, returns from exploration are systematically less certain, more remote in time, and organizationally more distant from the locus of action and adaptation" (March, 1991, p. 73). Exploration refers to those activities or routines that are learned from playing and risking new approaches and ways to do things, refers to learn from the process of experimentation and creation (Raisch \& Birkinshaw, 2008).

\subsection{The difference in the nature of exploitative versus explorative activities}

Ambidexterity suggests the balance and the deployment of exploration and exploitation as part of the strategy. But, the problem here is that both activities are competing for time and resources of the enterprise. Moreover, both type of activities demand such different kind of skills in persons and type of processes and sometimes leadership from top management. That represents a challenge to the different firms, which are realizing that in order "to be ambidextrous, organizations have to reconcile internal tensions and conflicting demands in their task environments" (Raisch \& Birkinshaw, 2008, p. 375). That is why "ambidexterity is associated with numerous difficulties, since the two innovation strategies, exploitation and exploration, do not only have different characteristics in terms of timeline, risk and potential return, but also call for distinct organizational structures, processes, cultures, and capabilities" (Ghemawat \& Costa, 1993; Tushman et al., , 2004) referred by Schulze, et al., (2008).

Besides the challenge of reconciling both activities, there are certain behavior regarding to ambidexterity that could lead to problems and negative performance. We discussed that, in general, achieving ambidexterity is important for the firms to 
face current fast changing environments; then we can infer that the lack of concern from top level management about avoiding one of the activities could be negative. Nevertheless, also the excess of time devoted to either, exploration or exploitation, could represent a problem for the organization entering in a negative vicious circle (Raisch \& Birkinshaw, 2008). On one side, if the majority of the time and resources of a firm are dedicated to exploitation, then that firm could be weak for addressing the environmental changes (Raisch \& Birkinshaw, 2008). On the other side, according to Volberda and Lewin (2003) referred in Raisch and Birkinshaw (2008, p. 377) "too much exploration may enhance a firm's ability to renew its knowledge base but can trap organizations in an endless cycle of search and unrewarding change".

\subsubsection{Different ways of Achieving Ambidexterity in the Organizations}

One important question that scholars have studied in the theme of ambidexterity is related to which organizational arrangements could promote or inhibit to become ambidextrous. Approximately 40 years ago, Duncan (1976) proposed that for a firm to be ambidextrous it means that it should find separated structures that performs exploitation and exploration separately.

In the case of structural ambidexterity, it "argues that organizations can become ambidextrous by organizationally separating exploitative and explorative activities while at the same time establishing a planned level of integration. The partitioning of exploitative and explorative tasks allows for a perfect adaptation to the specific needs of the distinctive environments. The integration ensures that all subunits act in accordance with the overall organizational goals" (Schulze, Heinemann, \& Abedin, 2008, p. 2).

On the other hand," contextual ambidexterity is the behavioral capacity to simultaneously demonstrate alignment and adaptability across an entire business unit. Alignment refers to coherence among all the patterns of activities in the business unit; they are working together toward the same goals. Adaptability refers 
to the capacity to reconfigure activities in the business unit quickly to meet changing demands in the task environment. By their nature, such capacities are complex, causally ambiguous, widely dispersed, and quite time-consuming" (Gibson \& Birkinshaw, 2004, p. 209). According to Gibson and Birkinshaw (2004, p. 211) "When contextual ambidexterity has been achieved, every individual in a unit can deliver value to existing customers in his or her own functional area, but at the same time every individual is on the lookout for changes in the task environment, and acts accordingly.

We can deduce regarding to both type of ambidexterity, that structural is based in creating different structures and focusing each either in explorative or exploitative activities. Otherwise, in the case of contextual, the responsibility of developing both type of activities is within the same unit, "building a set of processes or systems that enable and encourage individuals to make their own judgments about how to divide their time between conflicting demands for alignment and adaptability" (Duncan, 1976; McDonough \& Leifer, 1983; Tushman \& O’Reilly, 1996) referred in (Gibson \& Birkinshaw, 2004, p. 210).

In sum, we address the main topics and issues related to the ambidexterity theme, that would be useful for this dissertation. There were some, for example the way organizational learning promotes ambidexterity or the concept of ambidextrous leadership that are beyond this study. Nevertheless, it is important to consider that the way the firm behaves regarding to ambidexterity is a consequence of the behavior of the leaders.

\subsubsection{CONCLUSIONS}

Resource Based View of the firm and Dynamic Capabilities have emerged as dominant paradigms in strategy in the last 20 years and are focused on those resources that can provide a sustained competitive advantage to the firm. In this sense, it may seem quite logical to take for granted that the RBV can produce 
competitive advantage to the firm; nevertheless, there are some assumptions and implications that allow an open door to criticize the RBV approach, the main objection is related to the difficulty found in defining and measuring resources that can provide SCA; in the end, necessary attributes like rarity and value are often difficult to define in an objective way.

According to Professor Barney, "resource-based models of strategic advantage may need to be augmented by theories of the creative and entrepreneurial process" (Priem \& Butler, 2001a, p. 64). In this sense, incremental knowledge and creative destructive processes are very important in generating new VRIN resources that could give sustainable competitive advantage to the firms.

Despite all authors in favor or against the RBV on the firm, a very important question must be raised: Is the RBV strategy here to stay? Wernerfelt (1995) answers this question through a fabulous analogy that involves sports and firms: all games have a body of strategy knowledge which is independent from the specific opponent; however when a specific opponent is to be faced, one can tap into a different body of knowledge in such ways that differences can be exploited. In other words, one can do better by taking advantage of the firm's differences and diversity. Unlike sports, firm environments do not have enough opportunities for second divisions, therefore "strategies which are not resource-based are unlikely to succeed in such environments" (Wernerfelt, 1995, p. 173).

There are more areas that are found directly related with RBV and DC, for example knowledge management (Grant, 1996), strategic leadership, strategic marketing (Fahy \& Smithee, 1999), just to mention some. The more turbulent the environment of the industry, the more complex to maintain a long lasting competitive advantage. In those conditions, there is an important type of advantage that is derived directly from RBV and DC, the named Transient Advantage. According to the anatomy of transient advantage, any competitive advantage goes through a life cycle composed of different stages (Gunther McGrath, 2013). But the most interesting thing about 
Transient Advantage is the new rush dynamic perspective that this approach is revealing derived from the todays forces in industry, such the digital revolution, a "flat world", low entry barriers that boosted globalization, the near zero cost opportunity for getting information, etc. Quoting from Gunther McGrath (2013, p. 64) "in a world where a competitive advantage often evaporates in less than a year, companies can not afford to spend months at a time crafting a single long-term strategy. To stay ahead, they need to constantly start new strategic initiatives, building and exploiting many transient competitive advantages at once".

Regarding to ambidexterity, decision-makers must find the way to cope with those activities, in order to provide the firm with a healthy operation and at the same tie, with a long term vision that allow the firm to prevent changes in environment that could jeopardize the survival of the organization. 


\subsection{INDIVIDUAL DECISION MAKING BY TOP EXECUTIVES AS A VALUABLE RESOURCE FOR STRATEGIC MANAGEMENT: A RESOURCE-BASED VIEW AND DYNAMIC CAPABILITY APPROACH}

Making good decisions is a complex task, even more in today's business world where a large quantity of information and a dynamic environment forces people to decide without having complete information. As Shafir, Simonson, and Tversky (1993) point out, "the making of decisions, both big and small, is often difficult because of uncertainty and conflict". Decision-making can be studied in different scenarios, since everyday activities involve making several decisions. In this section of literature review, we will explain a theoretical framework about individual decision making, specially focused in individual decision making in complex and ambiguous sceneries made by top level executives.

In the first part we will briefly show the main issues related to individual decision making, focusing on the complexity of top-level management decision-making. Bounded rationality concept will be presented as well.

After the theoretical framework on individual decision making, we will relate top manager's individual decision making to two of the most important streams in internal resources view of strategic management: the Resource-based View (RBV) of the firm and dynamic capabilities (DC), both of them focus on the optimization of the role of internal resources and capabilities as the principal basis for a sustained competitive advantage (SCA). The second part of the paper also briefly explains the bases of the RBV theory and also dynamic capabilities.

Later, we will continue exploring the difficulties for high-level position decision making so as to analyze whether these executives could be considered as valuable and unique internal resources for the RBV of the firm and enablers of dynamic capabilities. Several examples and different pints of view will be provided. And finally some conclusions are commented. 


\subsubsection{STRATEGIC DECISION MAKING: CHALLENGES OF INDIVIDUAL DM}

Making correct decisions is neither an easy nor a quantitative issue, more over, depending on the extent, the importance, and the magnitude of the consequences; decisions should be made with different approaches. In this section, lets explore some particularities regarding to individual decision-making. The aim of this section is to have a general view of the complexity and in somehow the ambiguity of decision-making process.

The more choices and variables related with the decision the more complex to take one of the best ones for the company; also Sieber and Lanzetta (1964) found that in complex decisions or in ambiguous sceneries the effort applied to take one is increased; even more "time devoted to information acquisition and amount of information required are related to the degree of response uncertainty generated by a problem, time pressures, and cost of information. The day to day work of a manager is composed by several individual decision making sceneries, and the sum of all of them and the results (positive or negative) will lead to the performance of the firm. In that way, analyzing the way they take decisions is very useful in management nowadays.

In this paper we are most interested in theoretical frame of decision making but related with strategic decisions. "Central among strategic process issues is strategic decision making. It is crucial because it involves those fundamental decision which shape the course of a firm" (Eisenhardt \& Zbaracki, 1992, p. 17). But in a firm there are hundreds of decisions that are taken per week, and deciding which decisions are strategic and which are not is not easy. Shivakumar (2014) presents a conceptual framework that clarifies according to two dimensions, degree of commitment and the scope of the firm, which decision should be considered strategic and which do not. The degree of commitment is measured by the extent to which a decision is reversible and the scope of the firm is often taken to mean the firm's choice if 
products, services, activities and markets" (Shivakumar, 2014, pp. 79 - 80). This is a way of classifying strategic decisions in a normative frame. Nevertheless, deciding strategic and non strategic decisions could be more or less confusing and time consuming, top executives should have very clear that distinction among those decisions is crucial for the future of the firm. What is true is that medium and top level decision makers must have and excel results when doing decision making, and that any failure could have serious consequences.

\subsubsection{Individual Decision-Making in Controlled Sceneries}

The aim of this paper is going deeply into the topic of decision-making in complex and ambiguous sceneries. But, before that, we will quickly make some comments about individual decision-making in controlled sceneries.

When making decisions, Drucker (1967) points out that breaking down the process in different sequential steps could be useful to diminish the risk-taking judgments. The six sequential steps that the author claims are: classifying the problem, defining the problem, specifying the answer to the problem, deciding what is right, building into the decision the action to carry it out and testing the validity and effectiveness of the decision. This is a rational way of finding the best decision, but as we shall see, it is not always possible to have complete control over the situation, and in several cases it will be necessary to use non-rational methods, moreover in high positions in firms.

The above named methodology is applied to single situations when we are evaluating different possible decisions, but how to decide when we have several options and most of them appear to be convenient? How can we make trade-offs when comparing quite different situations? According to Hammond, Keeney and Raiffa (1998) this is one of the most difficult challenges in decision-making. They propose a methodology called even swaps, that "provides a practical way of making trade-offs among any set of objectives across a range of alternatives". This method 
forces us to think and evaluate correct objectives among different options and discriminate those who represent a bad decision in an iterative process until we get the best option.

The above recipe for individual rational decision-making could be applied in several situations and it represents a good reference frame when working on decisionmaking. According to Shafir, Simonson and Tversky (1993), a reason-based conception for decision-making has some good characteristics because "thinking of choice as guided by reasons provides a natural way to understand the conflict that characterizes the decision making".

Decision-making in controlled sceneries is quite rare in today firm environment. In general, decision-makers are more to use a combination of rational and irrational decision-making because all of the options and information about those complex day to day decisions are not available. According to March (1978, p. 588), "at first blush, pure models of rational choice seem obviously appropriate as guides to intelligent action, but more problematic for predicting behavior". Then several other aspects should be considered when making decisions. Most executives will not have the change to have controlled sceneries when making a decision. Then several considerations should be taken into account, some of them due to lack of information or complex and ambiguous sceneries and other due to the complexity of human behavior and the "non rational obscure side of humans". Lets explore deeply those considerations.

\subsubsection{Decision-Making in Ambiguous and Complex Sceneries}

But sometimes the situation is not as straightforward as we would want it to be; in a world where the quantity of information available is growing at an ever-increasing rate, decisions should be taken in short periods of time; therefore, complementary approaches must be available to support rational methods. Decisions in such sceneries do not obey to the concept of "economic man", which according to Edwards 
(1954) is a man who has three properties: (a) He is completely informed, in the way that he knows not only all the probable actions, but also what the outcome of any action will be; (b) infinite sensitivity; and (c) rationality, that is one of the most important concepts and that means that "he can weakly order the states into which he can get, and he makes his choices so as to maximize something" (Edwards, 1954, p. 381). Keep in mind this kind of men with such exceptional characteristics that will not apply for many situations in complex decision-making. In normative economics it is expected that persons assume more an "economic man" behavior, assuming that the economic actor is rational; normative economics "does not need a theory of human behavior: he wants to know how people ought to behave, not how do behave" Simon (1959, p. 254).

In complex situations, strategic decision made by top and medium level executives take a special relevance. Several dimensions about decision-making should be taken into account. In the next paragraphs, different and complementary approaches for decision-making will be examined, some of them combining the rational and nonrational dimension.

\subsubsection{Rational vs non-rational: Decision-makers limited by their bounded rationality}

The way top executives make decisions has been a broad field of study. According to Jones (1999, p. 318), "the behavior of a fully rational decision maker would be completely determined by the task environment. If we know the environment and the goals of the decision maker, then we may deduce the decision maker's actions. If, however, the decision maker intends to be rational but may fail, then we will need to know something about the cognitive and emotional architecture of the decision maker". The answer arising here is that: Is it appropriated that decision makers under ambiguous and complex situations attached to $100 \%$ rational procedures? Are there other variables that must be taken into account? How is it supposed to deal 
with those situations? Next some considerations about behavior and limited rationality in individual decision-making will be addressed.

In ambiguous and complex sceneries, some authors disagree with $100 \%$ rational and well-structured processes in decision-making. They alleging that decisions are made not only with the left rational side of the brain, but also with the right side (Mintzberg, 1976) and sometimes even trusting in gut (Hayashi, 2001). "In uncertain, ambiguous, or contradictory task environments, behavior is a function of goals, processing limits, and the connection between the decision maker's problem space and the task environment (objectively characterized). In this far more complex situation, problem-space representations may interact nonlinearly with goals and processing limits" (Jones, 1999, p. 319). According to Hayashi (2001), business executives support their important decisions using intuition and trusting their gut, both could be considered non 100\% rational approaches. Gut and intuition are directly correlated with experience. It is quite difficult to define what gut and intuition mean, but hearing what important CEOs say could help us to figure it out. According to Lutz, CEO of Exide Technologies in 2001, the most critical decisions are made "with subconscious, visceral feeling. And it just feel right" (Hayashi, 2001).

Non-rational decision-making is not a bad procedure or something that belongs to a small set of firm environments. At the end, decisions are going to be taken to satisfy certain necessities or variables, and doing in optimal way does not mean that they are necessary satisfying the decision maker. According to Eisenhardt and Zbaracki (1992, p. 35)" strategic decision making is bounded rational in that strategic decision makers are cognitively limited and engage in a cycling among rational decision making steps".

Greenhalgh (2008) shows a very good qualitative analysis about how business occupiers do decision making when deciding whether and where to relocate. Findings are very interesting because they support the non completed rational way of thinking and also quoting Greenhalgh (2008, p. 122) conclusions, "Rational choice 
equilibrium economics' notions of rationality and optimality rarely prevail in the complex and varied environment within which business occupiers go about making locational decisions. Some business occupiers do adopt approaches, strategies and decision-making processes that seek to reach optimal location decisions, subject to the constraints and conditions that any particular organization may find itself exposed to at a given time." Moreover in the same article, the author concludes that at the end decision-making is influenced by individuals and the higher the position in the firm the greater the influence.

Why is that some persons do not act as rationally expected when doing decisions? Why is that that they fail occasionally not demonstrating conformity to the classic expected utility model? That questions have been addressed mostly since the point of view of behavioral organization theory, then coming up with a very important concept in decision-making, bounded rationality. Jones (1999, p. 299) claims that "bounded rationality is a school of thought about decision making that developed from dissatisfaction with the "comprehensively rational" economic and decision theory models of choice". According to Jones (1999, p. 297) "bounded rationality asserts that decision makers are intendedly rational; that is, they are goal oriented and adaptive, but because of human cognitive and emotional architecture, they sometimes fail, occasionally in important decisions. Limits on rational adaptation are of two types: procedural limits, which limit how we go about making decisions, and substantive limits, which affect particular choices directly". Cecil and Jundgren (1974, p. 600) who cites Simon (1947) point out that "individuals and organizations cannot maximize decision making in an objectively rational way.

In most complex decision-making situation, an individual not possesses the knowledge of alternatives or the consequences or alternatives to select the one alternative that maximizes utility. Instead, the individual seeks an alternative that is satisfactory - one that is better than his level of aspiration". More precisely, Simon (1957) referred in Jones (1999) attribute the bounded rationality behavior to 4 specific characteristics of humans: (a) limitations due to the bounded cognitive 
ability and the complexity of the environment; (b) satisfying personally rather than optimizing behavior; (b) "the tendency to set aspiration levels for each of the multiple goals that the organism faces; (c) the tendency to operate on goals sequentially rather than simultaneously because of the bottleneck of short-term memory. As it can be inferred, decision makers are bounded not just because the lack of complete information for a rational evaluation of all possible options, but also because emotions and personal path dependence.

\subsubsection{DECISION MAKING IN MEDIUM AND HIGH LEVEL POSITIONS}

When talking about top executives and policy makers, not all problems are that simple, there is always a subset of critical, complex problems that must be solved. That kind of decisions are critical and most of us could think that they should be treated with the rational left side of the brain, but reality is quite different. Intuition, gut and the right side of the brain is needed; maybe this kind of magic could be the secret piece.

Quoting Etzioni (1989), "old-fashioned decision making does not meet the needs of a world with too much information and too little time. So-called rational decision making, once the ideal, requires comprehensive knowledge of every facet of a problem, which is clearly impossible today". Everyday simple decisions are prone to be solved using rational methods, where the simplicity of the problems invites us to solve them using a deep analysis of all the solutions. But making complex decisions require different skills and perspectives of the problem, and success will depend mostly on the people who are involved in decision-making.

As Hayashi (2001) points out, according to Ralph S. Larsen, chair and CEO of Johnson and Johnson, there is a huge difference in the way middle and high management make decisions. He points out that decision making in middle management levels is mostly quantitative and requires special brilliant management skills. The difference strives in the fact that, often, complex problems cannot be solved applying the same 
level of quantitative analysis that is typically used in middle level managers' less complex problems.

\subsubsection{Distortion of biases when making decisions}

When making decisions, there are several biases that can affect the process. As human it is almost impossible that we can take decisions taking out of our head all of our history and all experiences that shaped us. Path dependence is an important factor in the performance of managers. But not all experience we carry and not all the time.

But why is so difficult to avoid biases and other traps that could push us to take not the best decision? Maybe the response has to be with one of the characteristics of Resource Based View, the Path Dependence. According to the Resourced-Based View theory of the firm, it is path dependent because firm resources are directly related to firms' past activities, this can be determinant on increasing or decreasing growth through time. Resources differ in their impact on the firms' ability to generate profit or differentiation advantages, and hence, performance. When someone is making a decision several past experiences will shape that decision. Even more interesting, and supporting the importance of past experiences, is the fact that according to cognitive scientists, there are two modes of thinking: intuitive and reflective."

\subsubsection{Trusting in your gut}

As we saw, when referring to high level decisions and complex problems, not only instincts are key to problem solving, but also a correct balance in emotions and feelings is required. According to Damasio (Hayashi, 2001), "decision making is far from a cold, analytic process. Instead, our emotions and feelings play a crucial role by

helping us filter various possibilities quickly, even though our conscious mind might not be aware of the screening. Our intuitive feelings thus guide our decision making to the point at which our conscious mind is able to make good choices". 
But your gut is shaped by your past and experiences, which at the same time create rules and patterns that are immediately applied in decision-making. Those patterns are valuable in the decision making process, and as Hayashi (2001) points out, "the instinct genius that enables a CEO to craft the perfect strategy could require an uncanny ability to detect patterns that other people either overlook or mistake for random noise". Moreover, creation of patterns is related to two ideas: the varied and diverse backgrounds of the person, and the way they combine and make analogies of the varied fields of knowledge, to come up with excellent ideas and decisions, Hayashi (2001) calls this cross-index. "As people gain experience with a particular type of decision, they optimize the thought process they bring to bear" (Ariely \& Norton, 2011).

According to Hayashi (2001), business executives support their important decisions using intuition and trusting their gut. Gut and intuition are directly correlated with experience. It is quite difficult to define what gut and intuition mean, but hearing what important CEOs say could help us figure it out. According to Lutz, CEO of Exide Technologies in 2001, the most critical decisions are made "with subconscious, visceral feeling. And it just feel right" (Hayashi, 2001)

In the same line of clever decisions there is another combination that is critical for a good performance, the use of both the left and right side of the brain. It is common that managers and executives privilege the logical and rational left side of the brain, but practice leads us to a different perspective. According to Mintzberg (1976), it is acceptable for planners to have a well developed left side of the brain, but for top managers, a good balance and a well developed right hemispheric process is critical for accurate decision making. Planning and execution are systematic activities that demand capacities found in the left side of the brain, while the right hemisphere is in charge of other important abilities for top managers. 
Finally, I would like to introduce two interesting approaches for decision making that are related to non-rational decision making in today's world. The first one is the decision making approach, where Etzioni (1989) points out that decision making is more an art than a science, where executives need to make decisions with just partial information, due to the lack of time for a thorough analysis or the lack of information, and trusting their knowledge and intuition. The second approach refers to unconscious thought in complex decision-making that occurs when direct and conscious attention is directed elsewhere while trying to solve the problem in the unconscious level. According to Dijksterhuis, et. al. (2006) and his colleagues (Payne, Samper, Bettman, and Luce, (2008, p. 1120), "unconscious thought (a) is good at forming global or holistic impressions of alternatives (b) weights the relative importance of different attributes of objects in a relatively objective and natural way and (c) is less capacity constrained than conscious thought". These approaches combine the best of both worlds: the rational side of the brain and gut or intuition in order to make conclusions without all the information.

Personally, I do not thing that everything or the highest weight is attributed to gut and "the feeling" of the decision maker in gut based decision-making. A mental process and a hierarchical discarding process of different options for a decision should be applied, and the knowledge and "rational boundary" that every one holds is what will shape part of the decision. As it was already mentioned, according to Alice Gast business leaders should think like scientist; "a scientific mindset can inform and benefit the decision-making process outside of the laboratory" (Gast, 2015). Maybe the part that is most influenced by what we called "gut" is the fast we take decisions and the good that we perform when choosing among very similar decisions or those that lacks of information but has to be taken. In those situations gut and feeling of every one could be different and really represent a difference in the way of decision making is done. 


\subsubsection{INDIVIDUAL DECISION MAKERS AS VRIN RESOURCES AND ENABLERS OF DC: ACHEIVING PERFORMANCE THROUGH DECISION-MAKING}

Now that individual decision-making theory was addressed, we can realize that individual decision making for top executives is not a trivial process. It comprises a qualitative and a quantitative process and requires people with abundant experience, intuition, and brilliance in analytical abilities, among others skills. Thus, it seems that good top executives or managers that excel in the art of making decisions could be very valuable for strategy. Then good decision makers are valuable, rare, non-imitable and non-substitutable resources that can provide a sustained competitive advantage to the firm. Put in this perspective, it could be considered that good decision makers and their leadership could be a VRIN resource in a firm strategy according with the Resource-Based view of the firm. Lets explore and expand the last core idea, identifying why good leaders and decision makers in top-level management could be crucial for enterprises.

In this section there are going to be presented several cases that show how individual decision makers, since the point of view of internal resources strategy (RBV and Dynamic Capabilities), could represent VRIN resources or enablers of dynamic capabilities for achieving the final goal of strategic management: Sustainable Competitive Advantage. Diverse cases belonging to quite different kind of firms will be briefly presented. Citations could be used to going deeply in those cases.

\subsubsection{Top Executive Decision Makers as VRIN resources}

Let's explore the result of merging the theoretical framework on individual decision making with the theory of the RBV, to show why good individual decision makers in top executive positions could constitute a VRIN resource that can provide a sustained competitive advantage. What we are trying to show is how difficult it is to find characteristics of good decision makers in top positions. 
As it was already discussed, making good decisions in top positions involve several qualitative and quantitative characteristics from executives. They need to have great analytical abilities and an appropriate intuition that allows them to process a large quantity of information in a short period of time. In this sense, it is not easy or factual to find too many well prepared individuals that can have a great performance in different situations. Good balance in emotions and assertiveness is necessary (Hayashi, 2001).

It is possible to find out if an individual tends to use more the left side of the brain than the right side or vice versa. But using both sides on the right situation is essential for top executives when making a decision (Mintzberg, 1976). Moreover, great executives should know how to combine and use in the optimal way the left side or the right side, depending on the nature of the process or decision that they are examining.

Decisions are just one dimension, as Drucker (1967) points out "the most timeconsuming step in the process is not making the decision but putting it into effect". In this sense, a good decision maker could not only be a VRIN resource that generates competitive advantage, but could also generate more advantages when combining or using the different resources in the appropriate balance. Lockett, Thompson, and Morgenstern (2009) point out that by combining resources; firms are able to add value.

Lastly, path dependence and heterogeneity are crucial for VRIN resources in the resource-based view of the firm. Path dependence provides knowledge learned through years to the firm and heterogeneity ensures that any source of competitive advantage is simply a rent conferred by one or more imperfections in the resource market (exogenous variables) that prevent an equitable input allocation among competitors. According to Eisenhardt and Martin (2000), there should be an adequate amount of experience, because in some situations experience that comes 
too fast can overwhelm managers; on the other hand, infrequent experience can cause forgetting of what was previously learned. The fact that a manager's perception affects resource allocation assures that resources have plenty of different usages among different firms, supporting the heterogeneity theory that Barney (1991) held. In this sense, every decision maker in top positions depends on his path, his experiences, past jobs, and previous learning, that provide him with a unique characteristic that allows him to be different from others in the way he makes decisions. If we accomplish that, we can assure that unique experience of each person constitute a VRIN resource.

\subsubsection{Top Executive Decision Makers as enablers of Dynamic Capabilities}

There are several studies that aim to explore the role of dynamic capabilities in developing new ways of differentiation. Firms are facing rapidly changing environments and they need to move on as quick as they could improving product developing methods, marketing strategies, vertical scopes of integration, networks with competitors and partners, etc.; this occur specially in those firms that are somehow related with technological business where new technology continually change abruptly (Lavie, 2006; mentioned in Ellonen, Jantunen and Kuivalainen, 2011, p. 459). In this way they need to reconfigure resources, moreover those which are VRIN resources. In such a way, Dynamic Capabilities could be developed in hundreds of different ways, next we will analyze some ways of developing dynamic capabilities and how they are related with individual decision-makers.

Innovation and changing as Dynamic Capabilities are very important for achieving sustained competitive advantage. As seen in previous section, behavior and decision making process of top-level executives could activate or inhibit innovation in the firms. According with the natural hierarchical nature of companies, two kinds of capabilities could be found. Those set of capabilities are: first-order capabilities that comprises all that are done for the day to day operation (accountability, marketing, sales, and others that are operational routines and secondary capabilities, those that 
are needed for changing or renew the first orders (Ellonen, Jantunen, \& Kuivalainen, 2011, p. 460). Appropriated changes in correct time and correct form are needed with the rhythm of environment changing and are essential for adaptation. Dynamic capabilities represent the abilities of the firm to build new operational-level capabilities (Teece, Pisano, \& Shuen, 1997). In most enterprises the day to day operation (first-order capabilities) are essential for achieving objectives and surviving, but the second-order capabilities or operational routines are the ones that can set a big difference in the firm, because changing in the right time could represent the next competitive advantage.

In real estate business, after a qualitative study about how small and large business decide whether and where to relocate, Greenhalgh (2008) concludes with such interesting finding that is related with one of the main concepts in decision-making, the concept of bounded rationality; according to Greenhalgh (2008) determination of whether and where to relocate the business is one of the more complex problems and it differs from big to small business, but at the end individual decision makers in different positions in firms influence the process of decision making. According to him "It is concluded that, not only are the factors and variables that most influence location decisions of large companies different to those dominating the thinking of smaller firms, but also the processes they employ to arrive at such decisions. The evidence suggests that business occupier relocation decision-making rarely holds to the neo-classical assumptions that are required to fulfill the requirements of rationality and profit maximization. Small firms are more prone to making suboptimal decisions based on bounded information and constrained choice, but differences in the behavior of firms and organizations when seeking to relocate can also be attributed to their organizational status, culture and structure. To understand business relocation decisions we must recognize the influence that key individuals exert over both the decision-making process and its outcome. One more time, and in a complete different business among those treated in this paper, individual decisionmaking plays an essential role in getting competitive advantage for a firm. 
In regenerative dynamic capabilities, those that are needed to change and renew the firm's resource base, decision-making on top-level management is crucial (Ambrosini, Bowman, \& Collier, 2009). Certain traps in decision-making could be inhibitors of the deployment of this DC. Anchorage, framing and biases for example could represent three big enemies for decision that are intended to renew the base of resources (Hammond, Keeney, \& Raiffa, 1998); if decision makers are not open to new possibilities and are "anchor" or "framed" to past experiences he could be afraid of taking decisions or moving quickly when needed. Sometimes new people means new form of doing things and new ways of decision-making. "For instance a new CEO could be brought in, who has experience of transforming other firms, or strategic change consultants could be deployed. For such CEOs what they do within the firm is habitual - capabilities that they may have previously honed in different firms and contexts - and therefore these are not one-off performances. So for the CEO this is nothing new, only the context is new, but for the firm this would consist of a change in their dynamic capabilities, i.e. an instance of the exercise of regenerative dynamic capabilities (Ambrosini, Bowman, \& Collier, 2009, p. S19).

We could document more and more cases related with decision makers and decisionmaking processes as VRIN resources or as dynamic capabilities enablers. But it is not the purpose of the section. The purpose is to show how this is a reality and how the fact of doing research can throw valuable information that could be used for similar firms. In conclusions section, scholars are encouraged to apply qualitative methods to identify more of those cases in different industries. Decision-making is complex, but it can greatly influence internal resources and hence the performance of the firm.

\subsubsection{CONCLUSIONS}

As we already saw, individual decision-making is not trivial, particularly, in top positions it is not just about analytical approach, but of the combination of several factors and abilities. Abilities such as the right combination of the left and right sides of the brain, the correct use of intuition and gut, the effective application of logical 
and rational methodologies such as even swaps and the effective steps for taking decisions that Drucker (1967) proposes. Even more, effective decision makers need to go beyond conscious processes, looking for the holistic idea of every situation through unconscious processes that allow executives to take systemic decisions. Dijksterhuis, et al. (2006) resume the idea of making the right choice without deliberate attention, pointing out that "contrary to conventional wisdom, it is not always advantageous to engage in thorough conscious deliberation before choosing".

Practical experience and knowledge are also crucial for top managers when making decisions, this implies that decisions are "path dependent", as valuable and rare resources are path dependent to the experience of the firm. "One interesting possibility is that experience with making decisions helps people to find the right balance of thinking too much and thinking too little, and thus experience may be a way to improve decision making" (Ariely \& Norton, 2011). 


\subsection{STRATEGIC MANAGEMENT IN HIGHER EDUCATION INSTITUTIONS}

The purpose of this section is to explain strategic management in Higher Education Institutions and the involvement of top management in strategic decisions. In the last chapters deep literature review about Resource-Based View of the firm, Dynamic Capabilities and individual decision-making was done. In the context of Higher Education those topics are very important because universities do not scape from the highly competitive environment and structural changes in cost of information and competitiveness to attract new students. Literature review about strategic management and planning in higher education, as well as RBV, DC and decisionmaking will be explored. RBV and DC are very important trends in strategic management and both allow us to explore valuable resources and the performance of them in the firm; at the same time, DC approach permits us to explore the way base resource set changes in order to address the changes needed to keep being competitive.

As seen from experience ${ }^{5}$, top management positions in higher education are occupied by professionals in the areas of academics or research, some with entrepreneurial or practical commercial experience or vice versa, which could lead a dispute between members into which decision is more important: improve education quality? Or look for all the possible ways to keep student enrolment growing up? Is it possible to have both? In some point there's a need of a balance between offering a remarkable study plan for candidates and current students and the need of funding in order to continue providing a service. Either is a private or public institution it still remains a business, Washburn (2005) reinforced this aspect of the university as a constant seeker of revenue and therefore acted more businesslike than academic. Universities, and maybe all educational levels, today have

\footnotetext{
5 I have been working in Tecnológico de Monterrey University in México since 2004. The last 8 years I have occupied medium and high level positions as director. I have a lot of experience doing management at university and developing strategies in HEI context.
} 
adopted the language of business with the help of experts in the management area not necessarily because of their academic expertise. Some scholars argued that certain positions in Universities (mainly management and strategic decision making positions) should be occupied by people that have both academic and managerial skills. According to Washburn (2005, p. 204), "even university presidents were chosen for their ability to raise money and their close ties to the corporate sector". This combination is common to see nowadays in universities from Mexico that faculty is formed mostly of professors with business, engineering, etc. background in renowned companies rather than experienced in teaching (which could be a plus). Some campuses of Tecnológico de Monterrey for example, are composed in that way that $60 \%$ of the courses are thought by part time professors, those who have great practical experience because worked or are working in industry.

The higher educational sector faces very specific and peculiar conditions in relation to certain industrial sectors. For example, the main goal is to form professionals of high academic level, and under this premise, it is not possible to change this main activity. For this reason, one of the two questions that strategic management should answer in educational sector, according to Professor Kathleen Eisenhardt mentioned by Gary (2001) - Where do we want to go? -, if it is already answered, then the matter will be working to answer the second question -How do we get there?-, and to generate the strategy for achieving this goal, involves greater competitive intelligence than it was required a few years ago, since current threats are different and diverse. Moreover, Hughes and White affirm "the uncertainty from these threats necessitates that universities place a strong emphasis on improving effectiveness in how they structure, manage, and deliver these services to their constituents" (Hughes \& White, 2005).

According to Barrett (2010) the emerging threats that universities now face include but are not limited to: 
1. Shrinking enrollment,

2. Rising costs,

3. Demographic changes,

4. Online competition,

5. Increasingly competitive fundraising environments,

6. Accreditation pressures,

7. Recruiting needs,

8. Decreasing state and federal funding opportunities.

I would add at least two more threats that universities are facing right know: ninth threat, is the need of having community or international leaders in different industry areas as associate professors; and tenth threat, the downward trend of data price, I mean for students and for anyone, have access for good enough quality information on the web is possible almost anywhere.

Under the mentioned condition, the challenge seems to be enormous. Then... How can high management and education be related to offer a differentiated valuable service? The way top and medium level managers in universities apply strategic managements and the way they do decision making is essential for keeping the university well positioned under all mentioned conditions. Decision-making as part of every stage of the managerial functions (planning, organizing, leading and controlling) plays an important role, where especially top management is in charge of choosing the one right alternative to solve if not all at least most of the threats mentioned by Barrett (2010). 
In order to make easier to read, I will break down the rest of the section into three segments to better explain the implications of decision making in higher education. The order does not imply any importance about the topics:

1. Competitive Intelligence.

2. Organizational Structure and The trap of copying Incorrect Models or Strategies.

3. Decision-making and Leadership.

\subsubsection{COMPETITIVE INTELLIGENCE AND POSITIONING}

Business success is controlled by the influence of market and competitors and this is no exception for Higher Education Institutions. Nowadays competition is serious and powerful. Universities in the past had no trouble obtaining new alumni since they had a secure place in their geographic area (Barrett, 2010) or the advocacy of ex alumni that work as their public relationships representatives whether represented in a couple of parents encouraging their sons to study on the same family university or because of the lack of nearby competitors.

With new forms of obtaining a degree Universities are under a constant pressure to maintain or have a sustained growth academic top management need to work on strategic plans in order to survive in the market and obtain a part of the market share.

The Complex Problem of Getting enough Revenues

Complex environment, increasing in competition, improving in marketing skills, high differentiation are some of factors that have emerge or change nowadays and that put universities in a complicated scenery when trying to reach enough revenues for maintaining the day to day operation and moreover budget for medium and long strategic planning period. If to all this we add the fact that government funding is 
decreasing for some universities then it is clear that HEI must focus on strengths to differentiate. They must diversify the way they received funds, not only by government funding, but also with more local firms and international students (Cowburn, 2005). Washburn (2005) reinforced this aspect of the university as a constant seeker of revenue and therefore acted more business-like than academic.

Slaughter and Rhoades (2004, p. 14) referred by Barrett (2010, p. 27) indicate that higher education "[could] not ignore corporations because knowledge was not easily separable from the new economy. In the information society, knowledge was the raw material to be converted into products, process, or service" (Slaughter \& Rhoades, 2004, p. 15). On the other hand, Kirp (2003) also mentioned in Barrett (2010) stressed that priorities in higher education were not necessarily determined by the institution but by external constituencies such as students, donors, corporations, and politicians. He identified new business-type thinking within higher education in which departments were viewed as "revenue centers" (Kirp, 2003, p. 4).

Higher education institutions are businesses in the knowledge creation and management area and definitely there is a need to change the process of how they are being competitive. Name positioning is not a 100\% guarantee of success. Higher education institutions need to change their processes to collect, analyze and disseminate information more effectively as universities are encountering threats to their operations (Hughes \& White, 2005).

\subsubsection{Confronting planning versus action in Strategic Management in HEI}

Let's start again considering that universities are confronting a rapidly changing world and a tough environment, where top management decisions will be crucial for way they perform. Literature review shows that universities are better planning than executing strategies. For example, according to Cowburn (2005) European universities fail in executing what they have planned due to several situations and factors. Lack of a good and integral communication, a diffuse view of the mission and 
vision across different departments and areas of university, weak strategic differentiation and bad processes of decision making are some of the main reasons why universities tend to fail in implementation. Top-level management is responsible for all of the above mention reasons, and the higher lack of leadership in that level the higher of the fail in strategic planning results.

The question that remains here is how a university can generate competitive intelligence? Regarding to this question, Hughes \& White (2005) suggest that universities can use the following techniques in order to minimize competitive threats:

a) Benchmarking: universities need to analyze their internal processes in order to identify, understand and later adapt their strengths to competitor's best practices. One mistake that can be done is that organizations limit benchmarking to their own industry in this case Education. There's no doubt that HEI can improve their practices by taking a look at how manufacturing companies improve productivity with lean manufacturing for example applying "clean and clear" process for student enrolment or other school services. The use of technology and information systems is no longer exclusive of NASA or stock market, nowadays academic institutions depend on technology to offer their services and also to advertise and be known by future prospects. HEI have unique characteristics that made them especially complex. In such complex conditions, universities could be tempted to copy or just adapt different enterprise strategic management and planning models. They could think that it could work, that industry environment is more complex than HEI environment and that for those reasons copying and implementing industry planning and management could be a good solution.

b) Background checks: Further than asking for individual's references in former positions in other companies, universities need to be sure about the person they are hiring, in the case of Tecnológico de Monterrey and as part of its organizational culture, all employees must act accordingly to a code of ethics and personal integrity. 
c) Competitor assessment: similar to benchmarking practices, it is important to analyze direct competitors operational, marketing and academic activities of course. It is no secret that there is rivalry and a constant struggle between academic institutions in order to reach a higher number of new alumni but also Hughes and White (2005) suggest that institutions should know how well their competitors are dealing with legislation impacting higher education, or with regard to cost saving, and outsourcing decisions.

d) Win-Loss analysis: Activities involved in the area of customer service and marketing that will help obtaining and retaining alumni but also making an analysis of new fundraising methods.

e) Network analysis: Just like in multilevel corporations, everything is about making the right connection whether it is an experienced business owner in the community that can collaborate "in-company" sharing their work experience to students or the possibility of hiring individuals with richer experience in different business or engineering areas. Students value what they learn on a basic lecture it is a reflection of what happens in the day to day business basis. It is very common to start seeing part time professors working in HEI but also in their own companies.

Competitive intelligence is about mastering the techniques of how to use the information whether it is through network contact or benchmarking analysis, information is crucial for decision-making, hardest part is how to interpret information in order to create a competitive advantage.

\subsubsection{ORGANIZATIONAL STRUCTURE AND THE TRAP OF COPYING INCORRECT MODELS OF STRATEGIES}

Tierney (1988) points out that "institutions with very similar missions and curricula can perform quite differently because of the way their identities are communicated to internal and external constituents and because of the varying perceptions these groups may hold". Communication and even human skills that are required at every 
management position play an important role while planning and implementing strategy. Here I come to the question, if universities have similar goals and curricula why their position in the market is quite different? All comes back to communication and the importance of having the right individuals in the right position. From my previous experience ${ }^{6}$ in other higher education institutions a common problem was communication towards the external customer (students). Frustration was a common factor at the beginning of every semester for instance tuition fees increased during a semester and students were not aware until the first day of classes, when asking about the information, the personnel wasn't sure exactly how much was the increase. And without having that information students were not prepared economically to pay the different fees, this type of situations lead to frustration also to the parents who came asking questions and didn't receive a proper answer. Something learned from my professional experience is that if you don't know the answer look for it but never let the customer go away and worst of all with a problem on their hands. It's not the purpose of this document to talk about service delivery but still is an important area to discuss or analyze while planning differentiation strategies for the institution. "An organization's culture is reflected in what is done, how it is done, and who is involved in doing it. It concerns decisions, actions, and communication both on an instrumental and a symbolic level" (Tierney, 1988, p. 3).

In HEI, decision-making processes are also related and affected by the kind of organizational structure. According to Timberlake (2004), highly differentiated organizations tend to have bigger and more hierarchical charts, making that innovations happens in a more difficult way and slower. On the other side, highly integrated organizations are thinner in their charts and thus, presents less inertia to change and innovation (Dougherty (2001) referred by (Timberlake, 2004)). Obviously, the optimal organizational chart should be that one that seek both integration and differentiation, and that allows innovation in an easy way and at the

\footnotetext{
${ }^{6}$ I have been working in Tecnológico de Monterrey University in México since 2004. The last 8 years I have occupied medium and high level positions as director. I have a lot of experience doing management at university and developing strategies in HEI context.
} 
same time presents hierarchical organizations that allows different units to work properly.

There is extensive literature about the different models or ways of planning and strategic management for diverse companies, according to their size or activities. As mention by Lorange and Vancil (1976) every business has a strategic planning; however, the design of that process - to decide who does what and when and how to do it - can be complex, but vital to the success of the planning effort. According to Dooris, Kelley and Trainer (2002), the first significant formal meeting of higher education planners was a 1959 summer program attended by twenty-five campus planners at the Massachusetts Institute of Technology; from the 1980s through the end of the century, the visibility and volume of strategic planning in the academy continued to ascend.

Nevertheless, there is a huge difference among business world objectives and HEI objectives. According to Dooris, Kelley and Trainer (2002) for business world improving means getting more revenues and improving market position, for HEI improving is attached with more variables, such as academic quality, better professors, good facilities, good services, the best educative technology (Dooris, Kelley, \& Trainer, 2002). Spendlove (2007) also supports this idea, pointing that copying or adapting leadership models from industry to HEI could be not the most appropriate solution. For such mention conditions, it seems that Michael Porter's traditional definition of strategy cannot be applied in a step-by-step way. Porter (1980) suggests that strategy is a matter of positioning, finding a market sector where they can generate long-term profits, and then, when competition arrives, simply let it go and change the company activities. But, HEI cannot change their core activity - education -, instead they got to stick to the same activity. HEI then are going to be forced to be creative in order to maintain a position in the market. Barrett (2010) states that academic leaders face up big internal and external challenges, and they must guide their institutions in an intelligent way to remain competitive and relevant to the marketplace in order to succeed and survive. 
If copying or implementing industry strategies seems not to be a good option, what about trying to copy a HEI model. Concerning to this one problem arises: depending on several factors, there is plenty different kind of universities each with quite different needs. Several factors incise in it, for example: the university is public or private, is part of a consortium, its model is centralized or decentralized, is profit or non profit, the kind of leadership and decision making model, the KPI, etc. What is true is that, "in universities strategic management should be done with a permanent eye on their specific organizational environment rather than by an analysis of the applicability of yet another prescriptive model from yet another management school" (Tavernier, 2005, p. 1). Maybe HEI complexity points out to look for specific and tailor-made models of strategic management. According to Tavernier (2005, p. 15), "to find out what is really important for strategic decision making in colleges instead of relying on yet another prescriptive model of yet another management guru, it is much more rewarding to start out from comparative benchmarking and to look at concrete cases of decision making in some major universities".

Then the question arises... What are HEI looking for as strategy? The planning and strategic management for colleges and universities also will need to respond to the socio-political changes of the country and the region, such as the changes in the potential market of future students. Similarly, it will be essential to be aware of changes in competition, as pointed out by professor Michael Porter, who says that essentially, the strategist's job is to understand and manage the competition. Applied in this field of higher education, we can say "those institutions that were quick to seek information relative to their competitive landscape are more likely to have positioned themselves securely for future growth" (Barrett, 2010, p. 30). Then the question that we have to make ourselves again is: how we can apply planning and strategic management in order to achieve these goals? Or, how we can apply models as the one suggested by Porter (2008). 
But, what about skeptical people in relation to strategic management applied to universities? According to Dooris, Kelley and Tranier (2002), strategic planning is not uniformly applauded. Some have questioned whether it is a vital process, a core function, or the latest fashion in the technique boutique. I do not agree with it and I personally prefer Barrett's (2010) point of view which established that colleges and universities must know that adjusting to the changing landscape is a necessity, not an option. If they want to grow in next years, reputation is not longer enough; benchmarking, background checks, competitive assessments, win-loss analysis cases and recruiting appropriate academic leaders are essential elements (Barrett, 2010). In other words, well-applied strategy is going to be really important.

\subsubsection{DECISION MAKING AND LEADERSHIP IN HEI}

At the beginning of this dissertation, the topics of "decision-making" and "leadership" regarding to HEI where located in separate sections within the same chapter. As I moved forward, reading and documenting the main articles in literature review of those topics, I suddenly realized that most of the scholar's and most of HEI literature review was linking somehow both topics, decision-making and leadership. You could have an article with title related completely to DM and, in some moment the topic started to mix with leadership, and leadership were part of decision-making and the same happened for leadership title articles that finished talking about decision-making. Then I decided to address both topics in one section, this one. Along this section, the same phenomenon will be repeated, in some paragraphs dedicated to DM, suddenly leadership will came up, and vice versa. However, the most

important is to show the main literature review regarded to decision-making and leadership in HEI.

\subsubsection{Understanding decision-making in HEI}

Decision making process is a day to day operation in any firm at any operational level. Depending on the level and the importance of the decision the firm could be 
involved in several consequences for good or for bad. Individual decision-making could be such a complex factor where many variables related with rational, nonrational process and even behavior are involved. The way top executives make decisions has been a broad field of study. In this section decision-making and leadership related to HEI are going to be treated; further information about individual in complex and ambiguous could be found in the above section 1.3.

Higher Education Institutes are complex in their organizations, and in certain way are different from industry; this situation also challenges the decision-making process. In Section 1.4.2 it was argued that copying or emulating strategies from other type of industry would not necessary constitute a good idea or strategy. According to Tavernier (2005, p. 15), "to find out what is really important for strategic decision making in universities, instead of relying on yet another prescriptive model of yet another management guru, it is much more rewarding to start out from comparative benchmarking and to look at concrete cases of decision making in some major universities". In the same context of decision-making, Khefacha and Belkacem (2009, p. 55) very accurately point out that "the academic institutions are characterized by multitude levels of decision mingling consensus, negotiation, bureaucratization and hazard. As a consequence, the decision-makers are forced to take the appropriate decisions in order to respond to the expectations of a plurality of stakeholders constituting the university life".

There are several different proposals about the factors that influences decisionmaking. For example, Berardi \& Blackmore (2006) propose 7 steps: decision makers, decision situation decision criteria, the way of thinking rather problem or opportunity, decision support theories, tools or strategies, time and the people that is affected by the decision. Others scholars consider fewer factors, Atmosudirdjo (1987) cited in (Haris, 2012) just mention two: nature of organization and decision maker capacities. In the context of HEI, according to Haris (2012), who conducted a study in the State University of Gorontalo, Indonesia, five variables of factors were 
significant to decision-making process in the university academic managers. Putting them in order of significance the factors are the next:

1. The state of the organization,

2. The personality of the decision-maker,

3. The skills of the decision-maker,

4. The availability of information,

5. The external condition.

Despite being two or seven, from the different factors that were exposed, it could be that the individual decision maker is the most difficult factor to control (Haris, 2012) and, at the same time the most important because at the end he will be dealing and trying to control with the other factors; as Haris (2012, p. 33) claims "factor of individual or personal mostly corresponds to psychological aspect of decision-maker, whereas organizational factor deals more with environment or condition in the organization". Individual is always crucial when making decision, and is always affecting the dynamic of individuals and groups in organization and the dynamics of environment. As we shall see at the end of this section, leadership scenery is also important and complex for executives in HEI.

In HEI context, decision-making is often taken most by top-level managers. This is not different from other organizations, but at universities there are other special situations that conform a unique environment and that could complicate more the decision-making process. Decision-makers in universities should accomplish very special requisites that industries do not require: they should have academic credibility and experience (Spendlove, 2007), and at the same time they should be proficient with managerial abilities. In other words, at the same time managers in HEI must spend a lot of years doing academic career and developing their managerial 
and leadership attitudes and competences, maybe two activities that demand quite different capacities.

Despite some scholars argued that individual decision-making in top-level positions is important, it does not mean that those decisions should be taken without consultation, instead the decision-maker could take a participative approach that is always in favor of his leadership (Zermeño-Casas, Armenteros-Acosta, SologaistoaGuangorena, \& Villanueva-Armenteros, 2014). Those kind of leaders could make a big difference in organization; nevertheless, leaders that consider people as a part of the team, also gives the sense of empowerment where all together fights for reaching the goals of the firm. In large corporations, like Nike Inc. for example, employees in non-strategic areas are empowered to promote new ideas, actions for the benefit of their position and their company. At the end, something that human resources department value when hiring employees for a business unit or a higher position is the leadership of the employees when facing critical issues and how they come up with an innovative solution working in team. Most of the time some of the positions occupied at middle and top management were from employees that started at the bottom levels of the company like customer service or sales representatives. With this I would like to set up the example that decision-making is not exclusive for CEO's but in this case everyone working for the company with the single interest to contribute to the company and their personal growth 7 .

\section{Decision-making and leadership in multi-campus HIE}

In the context of multi-campus HEI, leadership gets more complicated because decision makers should align not just one but several campuses joined by one mission. Tecnológico de Monterrey is a multi-campus private university in México, which has embarked in a process of unification of processes and leadership around

\footnotetext{
${ }^{7}$ Comment from Fabiola Fierro, who worked for Nike Inc. (Mexico branch) from 2006 till 2010 as Sales tools coordinator and sales assistant. Fabiola have professional experience in advertising, management and merchandising in Mexico.
} 
31 different campuses since 2011. In this process, according to Salvador Alva, current President of Tecnológico de Monterrey University, the most difficult thing to do is alignment of processes and the establishment of a unified culture in the institution.

According to Timberlake (2004), "multi-campus managers must maximize the quality of both operational and strategic decision-making. Managing operational decision-making requires attention to timely service and timely decisions, while maximizing strategic decision-making requires planning processes, which create buy-in. According to Timberlake (2004), and regarding to leadership and decisionmaking process in multi-campus HEI, whether decisions are rational or non-rational decision makers are leaders with at least these three central components that guide their decision-making process: a) the importance of relationship building skills; b) the ability to manage participatory decision-making and its relational aspects and c) the ability to manage paradoxes or polarities. If we examined quickly the three aspects that remark Timberlake, we can note that all of them all related with relationships and with the ability to cope with ambiguity and the ability to defend your posture when needed and to collaborate with others postures when needed. According to my experience, those abilities are key in multi-campus HEI.

Finally, it is worth mentioning the two main challenges that came up with Timberlake (2004) study: on the one hand forces supporting autonomy and forces supporting centralization should be well managed by executives in multi-campus HEI. Being too autonomous or being centralized has advantages and disadvantages, and it is a matter of the leader to elucidate how to set the balance among the two, in such way that particular requirements of the local campus are reached and, at the same time, common processes of the university are fulfilled. On the other hand, "campus and system level leaders must be adept at managing relationships and participation processes" (Timberlake, 2004, p. 96). In the words of Tavernier (2005, p. 15), "attracting and retaining best staff and students requires an allocation system that rewards excellence, allows for decentralized decision-making and makes bottom 
up growth of centers of excellence possible. It is a delicate balance that can be reached not by top down command but by inspiring leadership of the flag waving type. Such steering is driven by a broadly shared vision over years slowly imbedded in the corporate culture of the institution".

\subsubsection{The challenge of leadership in $\mathrm{HEI}$}

In the same line of the complexity of the HEI executives decision-making process, but regarding to the topic of leadership, Smith and Wolverton (2010, p. 61) point out that "HEI present a unique set of leadership challenges. Leaders must balance the oftencompeting interests of these faculty against those of other constituents, including students, trustees, donors, government representatives, and community members". They also do an important contribution summarizing a new HELC Model (Higher Education Leadership Competences Model) which list the main leadership competences, which are divided in three subsets: Analytical leadership competencies (entrepreneurialism, creativity, strategic thinking and action); Communication leadership competences (oral and writing communication, assertiveness and the capacity to engage multiple perspectives in DM); Behavioral leadership competences (exhibiting lighthearted, unselfish behavior and strong focus and interest in people).

According to Ramsden (1998) referred in Pounder (2001), in universities leadership is about "tensions and balances". Tensions because the stakeholders which they have to deal, and balances because the soft skills that they need to apply in the day to day operation. As Kotter's (1990) and Ramsden (1998) point out in Pounder (2001), when talking about leadership-management necessities in HIE executives, managers must be capable that have leadership skills, such as capability of producing change, alignment of people, and self-motivation. Other important topic that according to him constitutes the central requirement of managers in HEI is flexibility. "Such flexibility is reflected in a leadership approach that combines both the transformational and transactional dimensions" (Pounder, 2001, p. 283). 
The lack of alignment in the organization, could be more matter of leadership than decision-making, and is happening in universities because some managers and directors lack from abilities to delegate (Spendlove, 2007) and establish participatory ways of working. Strategic management and strategic decisions should not be isolated at the top of the organization. According to Eisenhardt (1999) and Worley, Hitchin and Ross (1996) mentioned in Timberlake (2004), distributing strategic decision-making is better because all people can be aligned and be part of decisions and the commitment increases, versus traditional models of strategic management take into account their executives to analyze the environment and the organization to make decisions (we strength this with the Nike Inc. example above mentioned). Also most of those scholars argued that distributed leadership should be the most correct form of leadership in organizations, also in higher education institutions (Shattock, 2003) mentioned in (Bolden, Petrov, \& Gosling, 2007). Strategic decision-making should also be flexible in terms of including ideas from different departments of the organization. For transformational leadership it is very important to get involved most of the personal of the firm - in this case the University - in decision-making process. Related to this topic, Pounder (2001, p. 284), claims that "the participative decision-making involved in rational/collegial decision processes is a focus of the intellectual stimulation dimension of transformational leadership".

Making good decisions in top positions involve several qualitative and quantitative characteristics from executives. They need to have great analytical abilities and an appropriate intuition that allows them to process a large quantity of information in a short period of time. In this sense, it is not easy or factual to find too many wellprepared individuals that can have a great performance in different situations. Good balance in emotions and assertiveness is necessary (Hayashi, 2001). Assertive decisions made in high positions are crucial for the development of sustained competitive advantage. Good experience, combined with gut and the ideal handling between left side of the brain and the right side will provide this assertiveness. Finally, as Drucker (1967) said "an effective executive is expected to make decisions 
that have significant and positive impact on the entire organization, its performance, and its results characterizes the effective executive".

\subsubsection{Academic Leadership or Management}

Other very important factor that is affecting the skills of leaders in HIE, is the substantial change in the profile governance structures inside universities. According to Yielder and Codling (2004) the trends like the new world of information technology, the massification of students, the decreasing value of a graduated title, and in general the changes that HEI are facing nowadays, have had a deep influence on the way they are managed and the way DC is taken. According to Miller (1994), and Dearlove (1998) referred in Yielder and Codling (2004, p. 318 and 321) "the collegiate decision processes of the "old" universities no longer meet the needs of a modern institution, and are being replaced by a more corporate management model. In more traditional universities there is a tendency to promote elements of academic leadership at the expense of management, while post-modern institutions with a more youthful heritage tend to emphasize the latter at the expense of the former". Yielder and Codling (2004) also claim that universities that are more traditional incorporate academic leadership in which management positions and management duties occur "almost incidentally depending on the personal qualities of the professor and administrative staff" (Bowen \& Shapiro, 1998) referred in Yielder and Codling (2004, p. 321).

According to my experience and the current context of HEI, the above paragraph makes the big difference and sets a new era in HEI. A great paradox is happening in universities and colleges, I will explain next: managerial activities (that add little value to the core business of university - education -) are incrementing day by day due to the complexity of environment that has been mentioned in several sections in this work; then, those positions are full time and, when occupied by academics they will not have any time for teaching or doing research; if they do not, academic credibility will diminish and hence their leadership and credibility. But, at the same 
time it seems for many universities (at least traditional ones according to Yielder and Codling (2004)), it is not appropriate that managers with non-academic credibility occupy those positions related with the administration of HEI. What could be the solution? That is a really interesting question.

The above situation creates very unique scenery, which must be taken advantage. It seems that there is not turning back, academic must cope with managers. The true is that "if managers do not value and incorporate the expertise of their academic leaders within management practices, leadership will be no effective" Yielder and Codling (2004, p. 321).

Bowman (2002) referred in Wolverton, Ackerman and Holt (2005, p. 228) points out, "the real work of academic chairs demands a diverse set of leadership capabilities: well-honed communication skills, problem-solving skills, conflict-resolution skills, cultural management skills, coaching skills and transition skills". Therefore we can conclude that in HEI strategic management, it is crucial the choice of the right people for the right positions. And it seems that, those positions for decision-makers, should be taken by academics with managerial and leadership skills, persons with an appropriated balance between the academic training and the management skills. 


\section{CHAPTER 3. METHODOLOGY}

This chapter presents all aspects related with the methodology followed to answer the research question and to address the general and particular objectives. The chapter is structured as follows: first I established the approach and the research strategy that were used for guide the design and execution of the study. After that, the methodology is explained in detail: unit of analysis, data collection, semistructured interview design and the specific analytic process used to address the research question and objectives. Finally, the procedure to assure validity and reliability is detailed.

\subsection{APPROACH}

In order to study how risk and uncertainty in decision-making and ambidexterity are related to strategic management, specifically dynamic capabilities and competitive advantage, I decided to use qualitative analysis as the best methodology to approach an exploratory study. I think this approach offers the possibility to address this topic that has barely research in the field of management and higher education. In this regard "the logic of phenomenological analysis is more likely to be assumed since qualitative researchers tend to regard social phenomena as more particular and ambiguous than replicable and clearly defined" (Van Maanen, 1979). Thus this is a qualitative, exploratory and phenomenological study.

\subsection{RESEARCH STRATEGY}

This section is aimed to present the research strategy that was used to address the research question that guides this dissertation and that let achieve the research objectives. It is very important to select the correct research methodologies for the empirical investigation. According to Easterby-Smith, Thorpe and Jackson (2008) there are at least three important reasons behind it: clarify the way research design 
is done; elucidate which design could work and which not; and third identify new designs that may be outside of the past of the researcher. Also the nature of research questions and the level of control that the researcher has over behavioral events should be taken into account (Yin, 1994),

Qualitative approaches are very often used for addressing "how" and "why" questions and, in the case of this research both, the research question and the general and specific objectives, are those type of questions. Despite both methods (quantitative and qualitative) are widely used, some scholars agree that qualitative methods are the most appropriate for studying topics related to decision-making and leadership. Complementary to quantitative methods which are closed-ended approaches, qualitative methods enable the researcher to look for emergent themes by combining the analysis and corroboration of the information found in the participants and the depth understanding of the world as seen through the eyes of the people who were studied (Okendu, 2008). According to Martinez (2008) qualitative methodology is vast used for studies at leadership level in higher education. Meanwhile, O'Mara (1999) referred in Greenhalgh (2008, p. 113) claims that "the only way to truly understand the reasons for certain management decisions is to go straight to the decision makers and ask Why? And How?; therefore interviews form the basis of investigation". On the other hand, some scholars encourage the use of various approaches for studying the topics of Resourced-Based view and dynamic capabilities (Lockett, et al., 2009; Ambrosini and Bowman, 2009). Ambrosini, Bowman and Collier (2009) claim that qualitative analysis could be better applied for identifying resource creation and regeneration process in dynamic capabilities. Even more, qualitative research is appropriate because those capabilities and resources have been poorly specified and because of the difficulty to observe and measure of VRIN resources and dynamic capabilities. Similar situation applies for decisionmaking in ambiguous and complex sceneries when not just rational thinking and the logic of scientific empiricism are significant, but also more ambiguous variables like behavior and gut of the decision makers. 
In sum, qualitative approach was selected for this study because the above reasons and because this methodology represents "techniques which seek to describe, decode, translate, and otherwise come to terms with the meaning, not the frequency, of certain more or less naturally occurring phenomena in the social world" (Van Maanen, 1979, p. 520). Regarding to qualitative research there are many several different approaches and types. According to Creswell (2013) approaches are categorized in five main different types: narrative study, phenomenological study, grounded theory, ethnographic study and case study. For this study, and after analyzing each one, it is clear that it corresponds to a phenomenological study. A phenomenological study "describes the common meaning for several individuals of their lived experiences of a concept or phenomenon" (Creswell J. W., 2013, p. 76). As pointed out by van Manen, (1990, p. 177), referred in Creswell J. W. (2013, p. 190) "the basic purpose in this approach is to reduce individual experiences with a phenomenon to a description of the universal essence". There are several features mentioned by Creswell (2013) that strengthen the use of this phenomenological approach next the following enlisted:

a) Typically interviews are the main way of data collection, just the way it is in this research;

b) Data analysis is based in narrow units of analysis, in order to move to broader units for answering the "how's" and "what's" (as we shall see in the next section this is what happens for this research, moving from quotations to complete themes in the process of analysis);

c) In some cases, researches seize their experience to discuss personal experiences with the phenomenon (due to the nature of the research, and in order to avoid some bias or non-supported comments, those experienced are mostly avoided, but they are used in the conclusion and future research sections).

d) The exploration of the phenomenon happens with group of individuals who have all experienced the phenomenon. 
e) The study concludes with a sum of the experiences of what they have experienced and how they experienced the phenomenon, and in this case, with a cross reference to literature review.

Semi-structured interviews were chosen as the main form of data collection. We will provide specific and detailed information about the method and process followed for developing the research process in the next section.

\subsection{METHOD}

\subsubsection{Sample and Campuses Selected}

The reason of campuses selection were deeply explained in Chapter 1, Section 1.1.3. Finally, the sample was integrated by 8 Division Directors and 4 Campus Directors, up to 12 directors for the first phase, the in-depth interviews phase, as we shall see in the next sections. Once we had the results of the first phase, and with the acknowledge of the supervisor and the academic peer ${ }^{8}$ (this acacemid peer was an expert in qualitative analysis with whom $d$, it was decided to apply 3 confirmatory interviews with higher level directors, in this case 3 more directors; the aim of this second phase was to confirm and explore some specific topics and responses found in the first phase, this increases validity and reliability as we shall see in Section 3.4. The aim of the sample strategy was to select the best sample of directors (as deeply explained in Section 1.1.3 in Chapter 1). This strategy allows the researcher to address the different questions and thematics from different points of view, and different campus circumstances and environments. Table 5A shows the sample from the point of view of the type of phases - in-depth and confirmatory - and Table 5B shows the sample adding the Category of campus selected.

\footnotetext{
8 In accordance with the supervisor, and to increase reliability, an academic peer with ample experience in qualitative research was consulted for decisions about methodology and also for reviewing the process of this dissertation. In Section 3.4 the detailed profile of this academic peer is discussed, as well as her participation in the process of dissertation.
} 


\subsubsection{Unit of Analysis.}

Understanding what is the unit of analysis is key for a correct process of research. According to Baxter and Jack (2008, p. 545) "determining what the unit of analysis (case) is can be a challenge for both novice and seasoned researchers alike". Considering the nature of the research question, the research strategy and the samples, and also responding to the question Who I want to analyze? (Baxter \& Jack, 2008), I realize that the unit of analysis is the quotations (fragments of the interviews that are assigned a code) from the participants' discourse.

\subsubsection{Data Collection.}

Data collection is very important for the study because most of the data is going to be generated through the process of the research. Semi-structured interviews are the main source of information, according to Creswell (2013, p. 79), in phenomenology "data collection involves typically interviewing individuals who have experienced the phenomenon". Next, the three different methods or data collection that were used for collecting data in the interview will be mentioned:

Semi-structured interviews: This will be the primary source of information. According to Rubin and Rubin (2012, p. 31)"the core forms of in-depth qualitative interviews are semi-structured and unstructured interviews". In contrast to structured interviews, semi-structured interview allows the interviewer to bring new ideas during the process (Rubin \& Rubin, 2012). This type of interview was selected due to the flexibility required, and because directors to be interviewed have quite different experience and curricula. Flexibility is also important which allows to jump from one topic to another during the interview. A general framework of the themes of interested will be the guideline for the interview, as it will be seen in the semi-structured interview design in next Section.

The interviews are divided in two types: in-depth interviews and confirmatory interviews, a deep explanation of both is going to be provided in next Section. Table 
$5 \mathrm{~A}$ shows how many of the different type of directors, rectors and vice-presidents correspond to the in-depth and confirmatory interviewees.

\subsubsection{Semi-structure Interview Design.}

Interviews are the primary source of information for this research. Semi-structured interviews were selected because this instrument allows the researcher to focused in knowing deeply the topics needed for the research. This instrument is also useful for phenomenology analysis, as Creswell (2013, p. 81) claims, "data collection in phenomenological studies consists of in-depth and multiple interviews with the participants". Next, the design of the semi-structured interview is going to be explained. It is important to mention that the design was done following the advices and part of the procedures and theory recommended by Creswell (2013) and Robin and Robin (2013).

First, it was decided to use open and ended questions. The majority of the semistructured interview is composed by open questions, which permit the respondents to use their own words and interpretations (Creswell, 2013; Krosnick and Presser, 2010). Due to the nature of the research and the complexity of the themes, those questions were the indicated for establishing an open and natural conversation with interviewees about the different topics, and at the same time allows the researcher to adapt the questions according to the experience, knowledge and preferences of the interviewed - part of the nature of semi-structured interviews according to Rubin and Rubin (2012) -. Questions were grouped in different categories, which facilitated the analysis and the development of the interview (Krosnick \& Presser, 2010).

The order of the questions were also important, serial order effects and semantic order effects were taking into account. The effects of the questions' order was rearranged after applying and discussing with the supervisor, the results and dynamics of that interview. In considering the order effects, it was decided to start the interviews with the strategy and the leadership questions, because those topics 
were the most familiar ones for the interviewees. After those questions, the sequence of the rest of the topics was addressed taking into account a semantic effect order, depending the context and content of the answers, and on the dynamic of the interview. For some interviewees risk and uncertainty was the next topic to treat, while for others was ambidexterity. Semantic effects order considers the coherently flow of the interview depending on how the related topics where group and the preferred order selected by interviewer (Krosnick \& Presser, 2010, p. 293).

Finally and after checking with the supervisor and an academic peer (Section 3.4 explains in detailed the participation of the academic peer), the final questions for the in-depth interviews are shown in Table 6. That interview was used to interview the 12 directors that belong to the 4 selected campuses. In the case of the confirmatory interviews, a reduced version was used. That version contained just the questions that the researcher wanted to verify, depending on the analysis of the 12 in-depth interviews. That reduced interview is shown in Table 7. Remember that, being semi-structured interviews, the questions of each section are limited and represent just an based initial set that will provide the plan for asking more questions depending on the plan and dynamic of each interview (Rubin \& Rubin, 2012).

\subsubsection{Methodology Procedure to address the Research Question and Objectives}

In this section we will explain the procedure that was followed for the data analysis. It is important to clarify in detail how the research was done, and how the data was analyzed in order to understand how the process was developed and hat were the implications during the research. As a general frame, and to facilitate the reading of this Section, during the process of the phenomenological qualitative research we followed the next specific steps:

1. Collecting Data 
2. Organizing and Preparing for Analysis

3. Codification Process

4. Data Analysis

5. Results and Conclusions

Those steps were defined taking as a frame of reference process defined by Creswell (2003, p. 197).

\subsubsection{Collecting Data, Organizing and Preparing it for Analysis}

After designing the semi-structured interview, and in according to my supervisor, a pilot study was developed with one medium level director at a different campus from the selected four. This pilot interview was fully recorded, emulating the conditions that were prepared for the in-depth interviews. The interview last about 48 minutes, and it was analyzed in detailed focusing mainly in the dynamic and order of the questions, and also in the content of the answers, checking if the idea of the question was correctly communicated, and also if the content of the responses will be sufficient for the research purpose. After validating the pilot study and checking it with the supervisor, it was decided that one test was enough, and that we have a good instrument for data collection. Now we were ready to do the interviews. First, it is very important to settle that all of the persons and interviewed were clearly informed about the anonymous process during the treatment of the information. All the interviews were done by the researcher because of two main reasons: first to assure the homogenous application and to assure the anonymity of the information. They were explained that random names would be assigned to them, in such way that after completing all of the interviews, they will just belong in an anonymous way to two possible sub-categories: either campus directors or division directors; all of the interviewees accepted this type of anonymous process. It is also important to mention that after checking with my supervisor and because in the process of qualitative research the context, the place and the interaction of the interviews are 
very important, it was decided to try hardly that all the interviews happen face to face (Rubin \& Rubin, 2012); fortunately all of the interviews happened that way.

There were a total of 15 interviews for this research, twelve in-depth interviews and 3 confirmatory ones. In-depth interviews were applied to the 12 selected directors across the four selected campuses. After analyzing those interviews it was decided together with the supervisor and the academic partner to interview three more directors who served as confirmatory interviewees in order to corroborate and check out certain patterns and responses that arose from the analysis of the 12 indepth interviews. Later in the next paragraph, we will explain deeply the aim and the way it was decided to implement the confirmatory interviews.

In the case of the twelve in-depth semi-structured interviews they were applied during 5 months, from April, 2016 to August, 2016. First, a special permission was requested from the two Zone Rectors whom the four selected campuses belong to; during this request the aim of the research and the value of the future findings and results were deeply explained. The request was done through email, and positive responses from the two rectors were obtained in 48 hours at most. After the permission from the highest management level, direct contact by phone call was established with the 4 campus directors in order to explain the aim of the research, to ask the confirmation of their participation and to ask permission for contacting their division directors. After the positive answer of the campus directors, a friendly email with an attached letter was sent to the 8 division directors. Through the letter, all of the division directors were asked for a possibility for a face-to-face interview and were informed about the relevance of the research and the anonymous treatment of the information. All of the directors confirmed their participation and provided three to four possible dates for the interviews.

Once the confirmation of all the interviewees was made, I arranged all the visits to campuses in the shortest period, in order to optimize budget and time. As part of the protocol for the interview, it was my decision to perform all of the interviews face to 
face in the director's office; I leave no possibility for a different format. I did it in order to impact positively the performance of the interview (Creswell J. W., 2003, p. 194) and also to improve the reliability and validity, as we shall see (Creswell J. W., 2003, p. 201). During three weeks I handled the visits to the four campuses in order to coordinate the three interviews at the same time for each one (one campus director and two division directors). Three of four visits occurred according to the date agreement, just in the case of one campus, the visit was change in the last minute because the death of one of the director's father to be interview. Table 8 shows relevant data from the interviews such: place of interview, length in time of the interview, date of the interview and length in words of the Interviews happened in a fluid way, most of the questions were answered without any conflict. Just in the case of three directors, three questions were incomplete, so they were required to answer those questions in audio and sent them by email; all of them accepted, and that way the interviews were completed.

Due to the length of the interview and the importance of remembering verbatim data, it was decided to record each interview, as long as we count with the confirmation of the interviewees (Creswell J. W., 2003). Each time an interview was done, it was transcribed in the same language into a Microsoft Word document with the help of two trusted assistants; after the WORD document was in Spanish, it was translated to English just to send to the supervisor in order to have feedback from each of the interviews; feedback from all the interviews were received in order to be sure of having enough information for the research. It is very important to remark that all of the qualitative analysis was done in Spanish, to avoid mistakes in the translation process, this was important for reliability and trustworthiness, as we shall see in this Chapter in Section 3.4. Just at the very end of the analysis, it was translated to English, and after that an English professor helped me verify the orthography and the grammar.

In the case of the three confirmatory interviews, they happened in the month of August, once the analysis of in-depth exploratory interviews was done and confirmed 
with my supervisor. The aim of those three interviews was to corroborate some patterns and results that were found after the analysis of in-depth interviews, especially in the topics of risk and uncertainty in DM and ambidexterity. The necessity of those interviews arose from a deep talk with the supervisor and with the academic peer, about different strategies for validation and reliability in the phenomenological qualitative analysis that was being implemented (Creswell J. W., 2003). The purpose of using this form of data confirmation is related to the concept of triangulation, in which multiple methodologies are used to study a phenomenon (Jick, 1979; Creswell, 2003; Denzin, 1978). In this case triangulation does not consists of multiple methodologies, but a creative approach that uses different ways - in-depth interviews, confirmatory interviews and KPI from each director - to confirm and be more confident about the results. The same in-depth interviews process of confirmation was applied to the confirmatory ones. The three interviews were done in a face-to-face format, more details can be seen in Table 8.

Due to the length of the interview and the different topics that are related to the research, and id order to increase the trustfulness and reliability ( (Creswell J. W., 2003 ) it was decided that the use of a qualitative computer program was important. After analyzing the different types of software (Denzin, 1978, pp. 808-810) and the availability at the University, it was found that ATLAS.TI software was an appropriated due to its nature as a code-based theory builder and conceptual network builder (we will see further that a code scheme analysis was chosen for the analysis). It is important to mention that the use of this software was verified with the recommendations from my supervisor and the academic peer who has long experienced doing qualitative analysis. In order to be proficient in software operation, an intensive 40 hours course was taken in January 2016. Among several advantages of using the support of a specific qualitative computer programs, the use of software contributes directly to a better quality of analysis and it could improve trustworthiness because "a computer programs allows the researcher to easily retrieve memos associated with codes, themes or documents" (Creswell J. W., 2013, 
p. 202). The interviews were uploaded in Spanish to ATLAS.TI and an hermeneutic unit was created in order to start to work with the data.

\subsubsection{The Codification Process}

The codification process started as soon as the first and second interviews were transcribed to a WORD document. As claimed by Rossman and Rallis (2012) mentioned in (Creswell J. W., 2003), coding process is about taking segmented text (sentences of paragraphs) and writing a word representing a category in the margins, in this case, using ATLAS.TI. After analyzing different ways of performing codification (Khefacha \& Belkacem , 2009; Haytko and Baker, 2004; Okendo, 2008; Voigt, 2014; Camacho-Ruelas, 2012; Creswell, (2013, pp. 327-346)), I realize that most of the codes had a few words, no more than five. I took the first two interviews as the initial ones for doing the first coding process and started to categorize and assign short descriptive words to each code, according to part of the process of codification proposed by Creswell (2003, p. 198). After finishing those two interviews, I had about 40 different codes but I realize that most of those codes refer to a broad general categories; for example the Ambidexterity, code could refer to different topics, for example, exploration, exploitation, the way of developing ambidexterity, risks, threats, etc. If I decided to use this code to refer to all the categories, maybe during the analysis I could lose hidden information, or simply it would be more difficult to perform and find the results.

After discussing it with the supervisor and the academic peer, I decided to use what I call "extended codes" which where codes inside the first list of general codes (like sub-codes) but that had the characteristics of being define by longer sentences that could help me remember what the segment was referring to, and thus could improve the reliability and the process of analysis. It is important to mention that when I refer to the extended codes, I will always used the "extended" word, meanwhile I refer to the regular non extended codes I will use just the word code. I proceed to codify the first two interviews, using the approach of extended codes. I went through both 
interviews about three to four times, trying to understand the meaning of the segments or quotations that could be assigned a code (Creswell J. W., 2003, p. 198). After finishing the codification process in those two interviews, I discussed the process and the extended codes with the supervisor and the academic peer. Once the feedback was taken into account I proceed to apply the extended codification process to the rest of the 12 in-depth interviews. After finishing I had about 487 extended codes.

Then I started the process of reducing the extended codes; I eliminated those that had 2 or less mentions and that according to the research do not provide valuable information and I joined those that were similar. After doing the reducing process, I finished with 326 extended codes. Then I proceed to gather them in the non extended format, where the name of the code was a few words long. A total of 29 codes arose, and were joined in three different themes: Risk and Uncertainty in Decision Making, Ambidexterity and Strategy. The codes and the themes where they belong are shown in Table 9. The themes are shown in the rows on black background with white letter corresponding to letter A, B and C, meanwhile the codes are numbered, from number 1 to number 29. Thus, codes 1 to 10 corresponds to theme A - Risk and Uncertainty in Decision-Making -; codes 11 to 20 corresponds to theme B - Ambidexterity -; and codes 21 to 29 corresponds to theme C - Strategy -. In the same Table, the number of extended codes that belongs to each code or theme is shown in the third column, and the number and percentage of mentions related to each code and theme are presented in columns four and five respectively. For example, for theme A, Risk and Uncertainty in Decision Makin, are associated 76 extended codes and 267 mentions of quotations, which represents about $28 \%$ of the total mentions of the in-depth interviews. As it can be seen in the Table, the three themes had a considerable percentage and number of mentions (above 28\%), in such way that the three themes are representative among the responses of the interviewees. The details of extended codes, and the way codes and extended codes are distributed among the interviewees are going to be presented in Chapter 4. 
During the codification process there were codes that were expected according to the questions of the interview and codes that emerge without being expected, but that reflects the importance of certain topics among the interviewees. Both codes are normal in a qualitative study (Creswell J. W., 2003, pp. 198-199) due to the nature of qualitative research. Different authors could refer to this type of coding in different ways, for example refers to different codes as theory-driven codes, prior-research codes or data-driven codes (Boyatzis, 1998); prior-research-driven codes are those expected codes derived from literature review and data-driven codes are those that arose from the analysis of the text, without expecting them. In the case of this research, those codes that were not expected are going to be referred, as "emergent codes" and the rest that were expected just like codes. This discrimination will be important in the further analysis and results Chapters.

\subsubsection{Data Analysis and Results}

Finally, data analysis and results are going to be deeply explained in Chapter 4 and Chapter 5 respectively.

In order to make the data analysis, and due to the nature of the phenomenology study, it was decided to use a narrative approach to convey the findings in the analysis. In the case of one of the final steps, the results, an interpretation of the findings in the qualitative research is done. The procedure mentioned by Creswell (2013, pp. 193-194) about phenomenological analysis also represented a based for the development of analysis. Regarding to results, also the narrative approach is selected. According to Creswell (2003, p. 200) to "researchers might describe how the narrative outcome will be compared with theories and the general literature on the topics". 


\subsection{VALIDITY AND RELIABILITY}

In qualitative phenomenological research it is expected that the experience and knowledge of the researcher play an important role in the process and the analysis of the results (Creswell J. W., 2013, p. 83).

Regarding to validation, there are several strategies that could enhance the validation of qualitative research. In this case, the selected strategies came from Creswell (2013, pp. 245-260) and those are: triangulation, peer review and clarifying researcher bias and Rich thick description.

Triangulation was used as a process of verification or contrast of information with the decision of the confirmatory interviewees; in this case, most of the results from the in-depth interviews were confirmed with the responses of confirmatory interviewees. One of the most important was the academic peer review, in this case with one of the most experimented qualitative researchers at the University, Dr. González'; it is important to mention that besides the supervisor, also Dr. González had the chance to review all the chapters of the dissertation, and she played the role of the "devil's advocate" (Creswell J. W., 2013, p. 251). Despite in qualitative phenomenological research it is expected that the experience and knowledge of the researcher play an important role in the process and the analysis of the results (Creswell J. W., 2013, p. 83), it is important to mention that the avoidance of bias was always taken into account, being aware of omitting valued judges or past personal experience; in order to minimize the bias because of personal interpretations during the interviews analysis, $100 \%$ of the interviews content was sent to the supervisor just to check with him that interpretations were bias free. Finally, regarding to rich thick description, it was mandatory during this research that all the interviews were recorded (provided that the respondent would permit), and that $100 \%$ of the

\footnotetext{
${ }^{9}$ Dr. Gonzalez has a Ph.D. Communication from the University of Seville, Spain. Her experience in qualitative research began in the late 90 s. Her works have focused on discourse analysis, specifically phenomenological studies and case studies. She has recently expanded her area of research into the virtual ethnography and the study of online communities.
} 
interviews were transcribed into text. The use of software ATLAS.TI also helped to maintain coherence across the process of codification, despite the large amount of information.

Reliability was cared by implementing a detailed process of codification, and also being exhaustive with the analysis of the data (Creswell J. W., 2013). The use of ATLAS.TI permitted the avoidance of repeated code or the avoidance of using similar codes. The fact that the program keeps the codes active every moment, allows to assigned correctly past codes, without the need of a paper codebook creation. Also the software allows the research to keep a "methodology diary" in which all the ideas and detailed were written down in order to be taken into account when backing to the analysis (remember that the analysis took several months). The last actions addressed the main issues related to reliability, on one side the detailed documentation of procedures (Yin, 1994) mentioned in (Creswell J. W., 2003, p. 201) and on the other side, the checking process of transcripts of the interviews and the codification process.

Finally, other details also contributed to the trustworthiness of this dissertation. First, the accuracy of data through the recording of the interviews and the credibility through a careful sample process and communication with the interviewees (Rubin \& Rubin, 2012, pp. 64-65). And second, with the decision of getting the support of an English professor for checking the writing of the complete thesis, and the translation of the interviews, helping to minimize any problem regarding to the process of translation Spanish - English and vice versa.

\subsection{SUMMARY}

In this Chapter the research methodology was detailed. First, the approach and research strategy were explained, detailing the reason behind the selection of an exploratory phenomenological qualitative analysis. The sample, the unit of analysis and the data collection were explained and justified. The process of design of the 
main instrument for data collection, the semi-structured interview was also explained. After that, the process followed for doing the analysis was explained in detailed, going deeply in the particular code process that was done for the research. Finally, validity and reliability aspects and strategies were pointed out and related with the process of research that was implemented. 


\section{DATA ANALYSIS.}

Now will analyze the three themes. The analysis process will be made following the most relevant codes and extended codes that will be useful to address the objectives and research question of this study; thus, not always the order of appearance of the codes its related to the number of mentions, but rather to the relevance for the thesis. It is also important to mention that during the analysis of the themes, when referring to codes and extended codes that belong to a specific Table, not in all cases the specific Table will be mentioned then, when the Table is not specified, is because I am referring to the very last referred one. This was done that way in order to avoid being repetitive with the references to Tables, which could cause annoyance while reading. Let us proceed with the deep analysis of each section.

\subsection{Analysis of Risk and Uncertainty in Decision-Making Theme.}

Risk in decision-making is another of the main themes for the thesis, which is directly related to two of the general objectives. The Decision Making part of the interview had the purpose of finding how interviewees cope with risk and uncertainty, how gut influences in DM and what is the role of all of these in the decision making process when developing strategy. Risk and uncertainty are important and give a touch of complexity in DM process (Shafir, Simonson, \& Tversky, 1993). For that reason, in the interview there were also questions aim to explore how prone were the interviewees to risk and uncertainty. Also it was desired to inquire about how fast or slow they make decisions and, at what extent they tend to delay or procrastinate because of an excessive analysis or, if they tend to be not cautious at all and make fast decisions without analysis. The interview also aims to explore in what extent gut plays an important role in the process of decision-making or even in the strategy of the interviewees. On the interview, the focus was put in those big decisions, those that create the difference in high and medium level directors. As Drucker (1967) 
claims, bid decision makers should be worried not for the quantity but for important decisions with high level of complexity.

Several questions of the semi-structured interview were related to this topic, and a big portion of the interview was dedicated to this. Despite this, it will be shown that there are many common responses among the interviewees, the way and the depth of the responses were different among the different directors, from those who showed preference to this topic to those who were confused, and seems that they have never cope with those scenarios. Considering a deep analysis of the interviews and the mentions or quotations related to the topic of risk in decision making -which comprises the way interviewees manage gut feeling and mistakes topics - a total of 76 extended codes were selected as the important ones for the analysis during the codification process. Working with such number of codes will be difficult for the analysis, then as it was mentioned in the methodology chapter, in order to simplify, I merge them in a reduced set of codes that were defined in order to address the objectives of the research. Table 10 shows the reduced codes (or codes) for the Decision Making Risk theme in detail for each interviewee; Table 11 shows the same Table but without the detail per interviewee. Meanwhile, Table 12 shows the extended codes belonging to each code in detail for each interviewee; the codes are indicated on black blackground with white letter, and below each code their correspondent extended codes are shown; Table 13 shows the same Table but without the detail per interviewee. Finally, all codes that were mentioned 4 or more times are indicated with a tight boarder just in the cell where the name of the code appear. This is the same format that is going to be used in the analysis for the rest of the themes

Going deeply in the Table 10, it can be noticed that the first four, John, Mike, Carl and James are Campus Directors, the rest are the Division Directors. As it can be seen, there are directors who contributed more than others, but in general there are not noticeable differences among the quantity of mentions among campus and division 
directors. John, Mike, Carl and James are the Campus directors and the rest are division directors.

The set of reduced codes is integrated by those categories that are useful to address the objectives of this research, and at the same time those that make sense according to the results of the interview. The way reduced codes where identified and joined obeys to two aspects: on one side to the objectives of the research and on the other side to the responses and how the semi-structured interviews were developed and the how the information was obtained (Boyatzis, 1998). Thus, a total or 10 reduced codes were extracted, which are showed in Table 10; code A in that Table (DM Fast Theme) refers to those quotations where rapidity seems to be important in DM; code $\mathrm{B}$ refers to anything related to boss decision-making and DM of high level directors; code $\mathrm{C}$ refers to the role that gut has in DM; codes D to $\mathrm{F}$ refers to quotations where the interviewees make reference to some situation where risk was considered; those situations were classified in the mentioned levels (high, medium-high and low); code $\mathrm{G}$ makes reference to how respondents react to mistake, either with themselves or with their teams; code $\mathrm{H}$ refers to those comments where respondents specifically make reference to the importance of checking before taking important decisions; code I refer to those comments where interviewees indicated something about DM among their team members, and code J refers to the style in decision-making. As it can be seen, in the last column of Table 10, the percentage of mentions of each code is directly related to the importance for the research, and also to the fact that there were or were not direct questions related to those codes. For example, for codes I and J, that represent 5\% and 3\% respectively, are very low because during the interview there were not questions directly related to these codes, they were emergent (Boyatzis, 1998); on the other side, codes relate to the level or risk of the interviewed - codes D, E, F and $\mathrm{H}$ - corresponds about 38\% of the mentions; other important codes are the ones related to how directors behave regarding to gut and mistake - codes $C$ and $G$ - which together represents about $25 \%$ of the mentions; finally, codes related to DM of high directors and respondents' bosses - code B represents about $18 \%$. It is important to remember that the extended process of 
coding obeys to a decision for improving reliability and to facilitate the process of revision from my supervisor.

From the analysis of the results we can see very important contributions to the theory of decision-making. We are going to start with the DM Risk Fast reduced code. When I referred to fast DM it is important the fast word no to be misunderstood. Fast is referring to agile decision-making, agile in such way that the directors are able to make decisions in their strategies that are needed in nowadays changing world, "strategic agility has become a real-life, hard-to-resolve contradiction for corporate leaders and their executive teams" (Doz \& Kosonen, 2008, p. 95). It is also important to remember that all of the interviewed directors occupy decision-makers positions and the interview is referred to decision-makers. This section will also contributed to analyze how fast or slow those directors act and how they "tread a fine line between ill-conceived, arbitrary decisions (extinction by instinct) and an unhealthy obsession with numbers, analyses, and reports (paralysis by analysis)" (Langley, 1995).

There were 10 different extended codes (codes A1 to A10) that were related to fast and decided decision-making subtopic and those codes contain a total of 29 mentions - see Table 10-. From the 12 interviewees, all of them except George and Laura, made some contributions. It is interesting to notice that campus directors are a little bit more prone to the theme of fast decision-making; 11 from the 29 quotations belongs to campus directors, which means about $38 \%$ of them, considering that just a third part of the interviewees were campus directors. Moreover, $100 \%$ of campus director talked about the topic of fast decision-making, while just $75 \%$ of the division directors talked about that topic.

In general, and analyzing the information contained in the quotations and the context of each interview, directors say that decision must be done, because it is one of the inherent part of their positions (extended codes A1, A4, A7, and A8 in Table 12). In the most mentioned extended code, extended code A7, seven directors claimed that decisions must be done, and that "it is better to apologize if you fail, but do it" 
referring that things must be done. It is not about just to think but to act, and it is very dangerous for the firms that things will not happen because directors are slow in taking decisions or because of the fear making decisions, but also they should avoid traps such anchoring or bias resources (Hammond, Keeney, \& Raiffa, 1998). Regarding to this, one of the campus directors, John, commented something very interesting:

John: "I hope that does not sound cliché but the truth that I have said always, is part of my philosophy of work and so tied well with these decisions, if ever I was going to run, that is to do and not stop do, then I think that is where I agree with what the institution in which work is at risk if we fail, it is for trying than by quitting."

It is very interesting that, despite some directors claimed that they always double or triple checked their decisions in order to revise whether they are or are not aligned to KPI or to their bosses, or just to verify with colleagues or bosses during the process of decision-making, they always claimed that it is essential to execute fast in order to avoid the typical situation of "paralysis for analysis" - it is a typical Mexican phrase that allude to those persons that spend a lot of time analyzing options while execution is delayed - (Langley, 1995). This is Carl's opinion

Carl: ... if necessary I will touch base with all entities that I need to consult, the president, the rector, the division directors, etc., so we are talking about high-impact decisions. I do not consider myself among those people that "executes just for execution without thinking". I use to have vision, but I do not like the dark, I like to see, and although it is not entirely clear, it if makes sense to me and I think it will worth, then I go ...

It is clear from Carl's comment, that it is necessary to check but at the same time to act. A third very important conclusion that arises is that attachment to regulation and norms in excess is clearly against fast DM, and in fact it makes the decision process slower (extended code A2 in Table 12). One of the interviewees even commented that he would not be able to work with a boss that would not promote an excess of regulation and cause analysis in the practical DM process against a practical and fast process. In this same topic another division director said that once he had a boss who 
analyzed every decision in such way that he looked for the minimum gain in every decision in such way that he always slowed down the decisions and he never had time for being strategic, maybe reaching the negative behavior of "paralysis by analysis" (Langley, 1995). James as a campus director went beyond exemplifying how this excessive attach to regulation could even affects the organic process of education in the classroom, he claims that in order for a professor to be innovative he needs to be free to decide and try different options, and than many times Tec de Monterrey University have privileged academic management and indicators over academic freedom:

James: I think often what we have done through the academic administration in college, is that we have privileged academic regulations and indicators, going against the teacher development as a person or as an inspiring teacher; so if a teacher wants to be very disruptive you say "Oh wait please, there are regulations you must apply two exams at least, and in previously schedule dates, you have to assign grades in a specific date, and also take attendance list to students each class"; then you start to decrease what makes us academics, that is the mental freedom to transmit knowledge in different ways.

As we saw in the strategy theme, some directors say that one of the big initiatives of the new president of TEC, who got the charge in 2011, is to achieve common processes for all campuses, where adherence to regulations and rules prevail. Regarding to that, it was found that some directors, Carl, James and Peter, think that being attached to rules always make the DM process slower (extended code A2 in Table 12), and it is complemented with the comment of Frank in extended code A6 in the mentioned Table, who claims that Mexicans are slow in DM, and other three directors who claimed in the same line, that they do not like a slow DM boss neither, those that are very attached to regulations. It is very important to remark that those last comments were made in a negative mood; like that situation had diminished the capacity of innovation, and thus exploration. When talking about ambidexterity, those directors believe that because exploration and innovation, are about risk, about exploring new ideas, new products, new academic programs (in the case of HEI), etc. it requires decisions and behaviors that give room to defying rules and changing 
them (Schulze, Heinemann, \& Abedin, 2008). In the same line, Peter is even more emphatic about this situation, pointing out (also in an annoying way) that he can loose that imagination and that capacity of execution that he had for solving problems and for offering innovative solutions that add value, just because the fear to fail in any regulation. Here is what Peter said:

Peter: ... today you protect more from mistake because administrative department is checking $100 \%$ of the rules to be accomplished, and ETHOS tool can be used to report you anytime you fail. Then the message is crystal clear, I must align myself $100 \%$ with the regulations and no errors are allowed, years ago it was different I was allowed to try new initiatives and I continuously said for myself "lets go, lets try lets see what happens, lets innovate",...

Another three directors pointed out that the fact that now everyone is asking for explanations, like why he did one thing or another, is very annoying and affects the operation and demands time and energy that it is not worth the time invested. Even more, according to Frank fast decision-makers are so relevant that employees are demanding fast decision maker's skills from the graduated students. As it can bee seen in Frank's comment, decision-making is one of the most important skills, because according to him Mexicans are slow in DM:

Frank: ... and this semester I visited a German company whose directors said: "regarding your students, what we most desire, is you to work in developing the skills of decision making, because it is a problem of Mexicans, I see here, Mexicans do not make decisions because of fear, because if you make a bad decision, you will be hanged ...

As we could see, for most of the respondents, decision-making should be made with caution but always trying to accelerate the process, and always avoiding "paralysis for analysis". It seems to be preferred to take a fast decision that avoiding taking it because the analysis is not $100 \%$ convincing. This is an important topic, because managers and directors are always making decisions that will impact the day to day operation. They should master it with the experience and time, and decisions should be taken quickly, another way Frank claims in his interview "we are going to be out 
of market"; this situation is being pushed by the fact that the world is fazing an ever and radical changing situation, and if facing this context means that strategies will be obsolete in the short time, then it is obvious that decision must be done (Peters, Reimagina, 2005) (Yielder \& Codling, 2004).

The analysis of the level that each of the directors claimed about how prone they were to take risk on big decisions, was very interesting. In order to obtain information more accurately, I did several different kinds of questions during the interview, and I also came up with different situations, depending on the context of each director and the way the interview was going developed. Again, a semistructured interview and the flexibility of qualitative research allowed it (Creswell J. W., 2003). After finishing all the interviews, and having a broad idea about how directors manage risk, I categorize codes according to the level of risk that they showed. The level of risk was grouped in three different reduced codes: DM Risk High, DM Risk Low and DM Risk Medium - extended codes D, E and F respectively in Table 10 -. Regarding to categories High Level (code D) and Medium Level (code F) both are very close in the intensity of risk, I just separate them from medium to high, when the interviewee was strong in the way he express the attachment to risk or when the risk was presented in situations that could jeopardize main indicators. From 79 mentions belonging to the three level of risk, 66 correspond to Medium and High, 32 for the former and 34 for the last, so no great difference is showed. On the other way, just 13 from the 79 quotations belong to Low Level, and in fact, the percentage of the mentions is just $5 \%$, one of the lowest in Table 10. That could show that in general, respondents are more prone to high and medium level risk than the low level in decision-making. Moving forward on the analysis.

In the high level category, 8 from 12 directors have some quotations or mentions related, but three of them, John, James and Peter, have 7 or more (see Table 12). It is also very interesting to notice that those three directors also scored high in gut, what seems to be a direct relationship between gut in decision-making (code $\mathrm{C}$ in Table 12) and high level of risk (code D in the same Table). Regarding to this three high 
level risk in DM directors, and analyzing the interviews, we can see that they are characterized because they are well aligned to the vision and values of the University, nevertheless they claim that despite they respect regulations, this is not the guide for decisions (extended codes D2 and D3). In that line, this is what John and Saul believe:

John: ... the objectives of my boss, and I align myself and my boss aligns with his boss, then at the moment objectives go down to me, does not mean that all do in fact there are some who tell my boss "I will not to this or this objective, it does not matter if that objective come from your boss", because I know that it is not going to be good for the campus or my team....

Saul: ... sure. I am convinced that in the long term, that change will bring us enormous benefits precisely because it is the prestige...

Interviewer: Would you be willing to risk your indicator for that change? Would you risk it? Saul: Yes of course I will.

The guide for decisions is what intelligence and the team decide in order to keep the campus competitive and always attached to the vision. They are willing to fail in KPI (Key Performance Indicators) or to say no to their bosses if they see that some action could be negative for their campus. Two are well-experienced campus directors and one an experiences division directors, John, James and Albert respectively. As Ariel and Norton (2011) claims, experience could possibly bring the right balance of thinking too much or thinking too little. Moving further with the analysis, it is clear that in those big decisions where risk is imminent, most of the directors claims to execute the decision because if they do not take the risk, then the competence will reach and maybe over passed them, they say that it is worth the cost (see extended codes D1, D6 and D7 in Table 12). In this line, this is what James believe:

James: What they will measure? Of course, that my ability to execute. What is waiting the organization from me? To take decisions ...

Finally, in high level risk, it is relevant that some directors again emphasizes about the importance of taking decisions no matter the risk, because in this rapid changing world it is impossible to be static, you have to move on an try different things, even 
more one of them talked about a culture of risk, inside the university. Let see what Peter said, when confronted to a bid decision and how Saul assume the risk even when he could failed in their indicators:

Interviewer: Yes please, can you mention a very creative or very innovative decision that you have be involved with...

Peter: Well the decision to offer $100 \%$ of the courses in English (when referring to one of the careers).

Interviewer: Then 100\% courses in English.

Peter: Yes, 100\% courses in English, I declared it without knowing the magnitude of the problem that was to come later.

Interviewer: Did you analyzed or was one of those decisions you said, we have to take it?

Peter: We just took it, otherwise we could be out of the market, you have to go forward and see how you do. At the end, we did not achieve the $100 \%$, but in the meantime we ended with better professors.

Saul: Of course. I'm convinced that in the long term this is going to bring us enormous benefits because that is what prestige is about.

Interviewer: Would you be willing to risk your indicator for this change? Would you take that risk?

Saul: Absolutely.

Interviewer: Interesting $\ldots$

We are going to leave the high level risk code, and start to analyze what was said regarding to those who where considered in the medium level risk. In the Medium level of risk category (DM Risk Medium reduced code) it can be seen from Table 12 that all of the division directors but James, have quotations related to this code, in facto $100 \%$ of division directors are more medium level risk in decision making according to the analysis, and Peter, Saul, Mary and Frank stand out due to the number of mentions, they are above five. In this category we are considering those mentions where decisions have an explicit risk, but always trying to make the decision whit care and minimizing the negative effect if something goes wrong. Respondents take risk, but seem to need some preliminary results or analysis that gave them at least a broad idea that the risk is going to be minimum or positive for 
success. They are not afraid to take decisions, but before taking them they tend to check before proceeding. As we shall see later most of directors tend to check prior making decisions. Referring to reduced code F (DM Risk Medium) in Table 12 we can see that some of the directors, in this case Mike, Frank and Saul, said that they try to be sure when taking vital decisions (extended codes F1 and F7 in Table 12). Lets see what Saul said:

Interviewed: Are you more risky person or risky averse?

Saul: I am part and part, there are occasions where experience tells you "this is the answer" and that's it, there is a little bit of intuition but this experience is behind you back ... Interviewed: Even with no formal validation, but something tell you that is going to work. Saul: if something tells you, and it is not life or death decision ... then you are going in.

George even talks about a culture of risk inside of Tec, but again a very calculated risk (extended code D8). Also, one of the directors - Frank - commented that employers are looking for decision makers that are not afraid of taking decisions, and in his point of view that is a problem because Mexicans are slow and afraid to make decisions. The most mentioned reduced codes correspond to those where respondents mentioned something related to medium risk or gave an example (extended codes F2 to F4 in Table 12); as can be seen, all but Mike and James have a mentioned to these codes. Related to those extended codes, directors mentioned put in the table several ideas, for example the necessity of taking risk in decision making because they are pushed to make risky strategies to address this complex and changing environment; Carl commented that he took the risk but not in "darkness" he likes to have some kind of light for DM; Frank emphasizes about the frustrating time with one boss that was not a risk taker at all. Some directors talked about risky initiatives in the way of teaching, and how some of those initiatives failed, and the relevance of the boss' support or the lack of it. Three extracts from different interviewees are added to show how this topic was handed:

Interviewer: ... and how do you consider yourself in risk taking? How do you see yourself? 
Frank: I take risks but I'm more cautious, big risk no. Yes, sometimes I break the rules and I am careful about this with my team. As a matter of fact, one of my colleagues is the one that tells me, "the good thing is that once you notice it you step out. So suddenly when there's a need of taking decisions about money and that but it is worth it, it is better to ask for forgiveness than permission and I used to do that a lot (referring to the fact that he executes). But yes, hey here is this rule, jump on it! And then if we fail well, then we ask for forgiveness.

George: Look, nothing is certain in this life, the most we can do is reduce the uncertainty through research, exploration, question, sometimes you do not get help or no one can give you his opinion, as the decision is taken with the information you have, and if that information is not sufficient for the probability of success or to reach a satisfactory level then do not enter there, and if you see that there is high probability of risk but the organization supports risk and tolerate the risk, then go.

George: ... and I do not take a decision if I'm not sure or if it is not validated, reinforced with another point of view. Of course, as time goes on I ask less and less opinion because I have experienced solving several cases; but I feel that one is good not only for what he has done but because of the context in which you are, and that context could facilitate decisionmaking.

Joe: It is a worthwhile risk (when referring to hiring international professors), we need to learn, but we need to make a proper selection of foreign professors, because we have a very successful case and two were not. Nothing happens, we already learned, we will try that all cases in the future will be successful. Perhaps it gives us a sense of urgency.

Interviewed: How well do you handle the issue of uncertainty and risk when you have to make a decision that can positively impact as many tough decisions but we are not certain? Joe: I like to take risks, but you have to measure the possible impact it may have that risk.

As we could see, most of the directors during the interview showed a high and medium level of risk, maybe that is the expected behavior considering the complexity of the current situation in the environment (March, 1991). But what about those directors who made comments in the set of low level risk. Those comments related to low level risk in DM were joined in the reduced code DM Risk Low (code E in Table 12). Three extended codes - E1 to E3 - composes it, occupying about $2 \%$ of the theme, and it is very interesting to notice that just three directors, Mike, George and Peter, 
did mentions related to this code. We can specially see from Table 12 that Mike has no mentions for high level risk and just one for medium level; and as a complement it is very interesting to notice that the two directors that report back to him consider that he is a low level risk person that do not performs well with those situations that may jeopardize any regulation or performance at the campus. In that extent, Mike comments illustrate very well a low level risk director, that in general tend to avoid most of those risky decisions. Here we have a fragment of the quote:

Mike: ... in what sense? Always attached to the institutional framework that is, if there is a decision that that implies risk in something that threatens the regulations then do not take it, yes, I am always an institutional director (meaning that he never defy a regulation, and always act according to them), we do have the institutional for better or for worse, all institutions are perfectible ...

As it is seen in the analysis of the Ambidexterity theme, those activities that aim to innovate and explore, requires some extent of risk and future prediction (March, 1991) and (Raisch \& Birkinshaw, 2008). In this line and regarding to Mike, one of his subordinates that belongs to the set of interviewed directors related that avoidance of risk with a lack of exploration and a much more exploitative behavior:

Interviewer: Ok that part, and it is what you see with Rodolfo, a leadership that let's you be you, but sets a guideline. And you see him more explorative or exploitative?

Albert: He is $100 \%$ exploitative he has never told me, do a vertical workshop, do a seminar, do this....

Interviewer: Ok and you do it yourself because of your own conviction?

Albert: because I'm very explorative.

As we saw, most of the respondents believe that decision should be taken in a fast frame of reference trying to avoid blocking decisions that could jeopardize the position of the University (Drucker P. , 1954). Most of the directors are consider high and medium level risk takers, but there is one that is more in the low lever risk. During the interviews analysis, I observed that directors, despite the level of risk that 
they manage, not just talked about the importance of velocity in DM, but also about the importance of checking the decisions, not just confronting with data but also with colleagues. Precisely, in this section we will analyze an emergent code that arises from that concern of the interviewees about the necessity of checking in DM process which is the reduced code $\mathrm{H}$ (DM Risk need to check) and their respective extended codes $\mathrm{H} 1$ to $\mathrm{H} 6$ in Table 12. It was non a planned but an emergent code, and it represents about $11 \%$ of the theme with a total of 30 mentions. The proportion of quotations linked to these codes is a little bit higher for campus directors than for division directors. It is also observed that $100 \%$ of campus directors mentioned at least two times codes related to the need of checking decisions, while for division directors one did not mention anything regarding to checking the decision making process. There could be a tendency in this higher position (campus director versus division director) to review and check decisions more in detailed, but with this data it is not conclusive.

This checking out in DM process, is more related to a rational and more structured process in decision-making Drucker (1967) and Hammond, Keeney and Raiffa, (1998), but it is important to analyze the content of the mentions in order to identify how those directors analyze and to what extent that checking and analysis could jeopardize the decision making, either making non analyzed rapid decisions or avoiding to take them because of the fear to fail. Lets analyze the content of the mentioned quotations or mentions.

The most mentioned extended code is the one that related the DM process with the necessity of checking before taking decisions. There were mentions from all the interviewees except for Joe and Peter (refer to extended code H4 in Table 12). Very interesting to check out what is the content and to what extent those directors made the comments. John for example, emphasized that the DM process is "not an solitary process", you must always have a support team and loneliness just bring as a consequence an increase in risk. Carl comments in the same line the next: 
Carl: Notice that I generally think that I weigh the risk, I mean I do take time to see what size it is and if I will assume or will not, or if I finally assume because we are an institution. If it is necessary to touch base I will do with all entities that you need to consult, it does not matter if it is the rector or area manager, we are talking about high-impact issues. I do not consider my self the kind of person that goes ahead just because, I do explore. Yes, I generate vision, but I need clarity I do not like the dark, but I have to be clear to see, and although it is not entirely clear, but you say, and makes sense to me and I think it's worth, then lets go.

Some of the directors also talked about being cautious and calculate risks. Regarding this, John was consider as a high level risk, he also tries to minimize risk, and Frank comments that even sometimes he brake the rules, he tends to be cautious. Lets see what John comments:

John: With this, what I want to tell you is that I'm the type of people that like taking risks, but trying to calculate them, I am not kamikaze, nor a hero.

Some directors also seem to take risk, even more when that risk does not imply to hazard a vital KPI (Key Performance Indicators) or when the risk is really worth it (extended codes $\mathrm{H} 2$ and $\mathrm{H} 6$ in Table 12) because that initiative or action seems to be critical and attached to their vision:

Albert: ... but I also think that regarding to this part, the year just finished, I happened to make difficult decisions because I had to reduce and cut staff ...

Interviewer: there was some risk in those decisions you made? If there was, Why did you took them?

Albert: Yes there were risks, but I have very clear where I want to go.

Interviewer: ok

Albert: then I know that sometimes you have to pay a price and if I have very clear where I want to go I can easily make a decision.

Sometimes, in between the complexity of certain decisions, the limitation of the time window and the lack of $100 \%$ of information, directors have to confront a decision with more than rational procedures or processes. In those cases experience, and something else related to intuition and visceral feeling enter in the equation, then gut 
plays an important role (Hayashi, 2001). During the interview, it seems very clear that several of the directors used their intuition when making important decisions, such as the opening of a new career, that for campus and division directors constitutes one of the toughest decisions. As a good example, and in such an important decision, Peter trusted in his gut, even when he failed:

Peter: Well the decision about studying a bilingual program, LIN and it didn't work a 100\%. Interviewer: Declared 100\%?

Peter: Yes a $100 \%$, without knowing the magnitude of the problem that was going to come later.

Interviewer: Didn't you analyze or was one of those decisions that you said, "we have to do it?"

Peter: We just took it, otherwise we could be out of the market, you have to go forward and see how you do. At the end, we did not achieve the $100 \%$, but in the meantime we ended with better professors.

The last example is related to the role of gut in DM. All comments of interviewees related to gut or intuition in DM are joined in the reduced code DM Risk Gut (code C and extended codes $\mathrm{C} 1$ to $\mathrm{C} 11$ in Table 12). In the same table it can be observed that 48 mentions were done for that code, which represent $18 \%$ of the mentions in this theme, the largest one. Most of the interviewees, 10 from 12, to be precise, said that gut is relevant for decision-making, for different reasons (see extended codes C5, C6 and C9 in Table 12). Campus directors were more active in their answers than division directors, because $100 \%$ of the formers made relevant opinions about this topic, while just $75 \%$ of the division directors registered some opinion in this topic. Interesting to highlight that one of the directors, Mike in this case, made confusing answers avoiding a direct answer about relevance of gut in DM; maybe because gut was not an option for him (Mike qualified low in risk level) or maybe because the concept of gut was not clear. Despite it, it is not correct to conclude with just one case, this is an interesting "coincidence" in the analysis. Here I would like to strengthen that it was possible to make this kind of correlation among different themes for one director thanks to the scheme of extended and reduced and extended 
codification, and to the support of ATLAS.TI software. Here the main confused comment is attached:

Mike: I will tell you I'm not sure if it is intuition but some people may see insecurity, I will explain from my point of view and I go back to the main reason, I don't have absolute truth, I have a group of elemental values and principles that fortunately go aligned with the institution but I don't have the knowledge or wisdom it's a tiny thing I say, that's what I learn in my PHD because with the PHD I became an expert in a dump of things I mean, after PHD I am the most ignorant of all. Those 4 volumes that are over there I wish I knew them all ...

Moving forward on relevance of gut in DM, an analysis of the quotations and selected codes indicate that directors perceived gut as important because they do not have all the knowledge, and in some occasions they need to decide without having all the information (extended codes C2, C3, C5, C6 and C9 in Table 12). Again the idea of a fast analysis and a revised process, is reinforced but adding the factors of gut and intuition. John has an important comment related to that:

Interviewer: I wonder what role this gut plays into decision making...

John: a lot, but it is an intuition that I do not consider making it alone, there are many managers who say that management position involves many lonely times, and I do not agree with that, because those decisions are difficult, and yes follow your intuition but check or verify first.

One of the director claims that intuition is related to the vision. According to John, intuition is not related to solitude and in difficult decisions is where you are supposed to check and consult colleagues and other persons, and at the end you would take decision, but not alone. Experience is another variable that was mentioned as it is directly related with the gut of a person; this could be related to the concept of path dependence in strategy (Lockett \& Thompson, 2001). Ariely and Norton (2011) also considers that experience in any decision optimize the process of decision-making, and could be also related to Resourced-Based View theory of the 
firm (Wernerfelt, A resource-based view of the firm, 1984). Lets see what is intuition according to Carl:

Carl: ... I think and how I see it for myself, gut refers to the set of experiences that you had, combined to those that you're living or seeing in your current scenario, and also combined with the dream or vision that what you have for the future ...

Some campus directors, consider that intuition and gut plays an important role when applying experiences from big and risky projects that positively impacted, and even those that fails. When gut was the drive for those directors it did not matter the risk or if that fails. Two examples of that are shown, the first one regarding to a big innovative project called Vertical Workshop where intuition of the decision-maker play a key role and the second one an example where the director trusted in the intuition of his past experiences:

Laura: ... I did not consider the stress that people would have gotten (talking about those professors that were burned out with the project), I just wanted to take them outside from their comfort zone, that was what caused stress and got sick.

Interviewer: And if you had known that the team may have sickened, would you still have done the vertical workshop?

Laura: Yes I would have done but perhaps in some other way.

Carl: Now I would tell you, because I'm not sure if it's going to be one of the conclusions you will come across with the analysis of this or subsequent interviews but, what I perceive is that in my position as a director, there is no way to avoid not bringing practices that were successful elsewhere, then this idea to ensure higher participation in media was something that took a lot of work, and if you say "Was media interaction the reason why your last position succedd?... well I don't know, it is just my intuition.

Other directors have similar and interesting opinions about gut. Joe considers gut as "capturing a dream as if it were a reality", while others like Carol expressed that for her it would be impossible to work with a boss who struggled when making decisions. Finally, Mary leave a good example about how gut works when pursuing a considerably good project: 
Interviewer: Did you make any market validation to verify if there was actually enough future students or not for this new bachelor?

Mary: When we changed from LEM (Marketing Bachellor) to LMC (Marketing and Communication Bachelor and the new one) no, we did not make any validation. Marketing Bachelor (LEM) was facing a stuck moment nationwide and we came up with a new proposal (LMC) and we just say "well must go for it".

When decision-makers are acting in the presence of risk, gut and bounded rationality, then strategic decisions are limited (Eisenhardt \& Zbaracki, 1992) and mistakes and faults could emerge. Part of the interest in the theme of Decision Making, was a research on how interviewees cope with mistakes in general. All comments and mentions regarding this topic belongs to the reduced code DM Risk Mistake and its extended codes (see code G and extended codes G1 to G5 in Table 12). Analyzing the codes we can realize that 8 from 12 directors expressed directly that they were aware that mistakes could happens as part of the process in decisionmaking. This is what Saul said:

Interviewer: What if they fail?

Saul: Well, they are in training process, I have children and also say "Well, fail now" and nothing happens if it is not a serious issue. When it happened and there was a learning experience, it is ok no problem at all. But, obviously it is not valid to commit similar mistakes again and again, you have to learn.

They also claim that it was very important not to repeat the same error twice, and always being in a continuous learning process from their mistakes. In general at least 5 of the directors, commented that there is no problem when you fail in something small, but please try not to fail in something big. Frank makes reference to that, mentioning one of the last University rectors:

Frank: Dr Rangel (a past Rector in Tecnológico de Monterrey University) would write about this in his book. He said, you have to make decisions and decisions are risky. If you make a small mistake it is fine, another it is fine but do not make a huge mistake... 
Other director's claim that, even when they failed in an important project, it is important for them to try achieving the project again, by modifying what necessary according to what was learned during the failed process.

Laura: Yes, I would do it again, but probably in less quantity, I wouldn't hired 6 professors in bachelor, I would hire just 4 professors with the profile we want and that is what we are going to do, I learned a lot from the experience that I had,

Finally, it is very interesting to point out that regarding to mistakes and failures one of the directors, John, claim that trust was the most important thing; he can tolerate failures, but cannot tolerate lies:

Interviewer: What happens if you are wrong, even if by doing this you say "I messed it up, the metric went down, I messed up in this I loose money"?

John: Nothing happens, we learn, it hurts. I'm not a manager that says well "good for you, you made a mistake" I am very competitive and I don't like making mistakes but still we do them,...

The level of risk of the interviewed bosses was not a topic for the interview. Nevertheless, with some of the interviewees the topic came out and also comments related to how high level directors should behave regarding to risk and uncertainty. Those codes related, were joined in the extended code named DM Risk Boss and High Directors (code B and extended codes B1 to B13 in Table 12). Next, a brief analysis and conclusions regarding these codes are going to be made. Extracts of the interviews are going to be omitted because the length if the analysis and the similarities to the above analysis. Going deeply in the analysis of the extended codes, we can see in general that most of the directors feel that they have support from their bosses for assuming risk and failures (extended codes B2, B3 and B8). According to 7 directors, they felt their boss support in general regarding those situations where they made risky decisions or where they failed. Even tough, 50\% of the division directors (4 from 8) said that their bosses manage a low level of risk, and 
just one campus director and one division director said that his boss manage a high level of risk. Just one director affirm in one comment that he felt his boss did not support him, and the same director qualified low the level of risk of his boss. Next I add two quotations, one from Peter and the second from Saul; both mentions show support from their bosses:

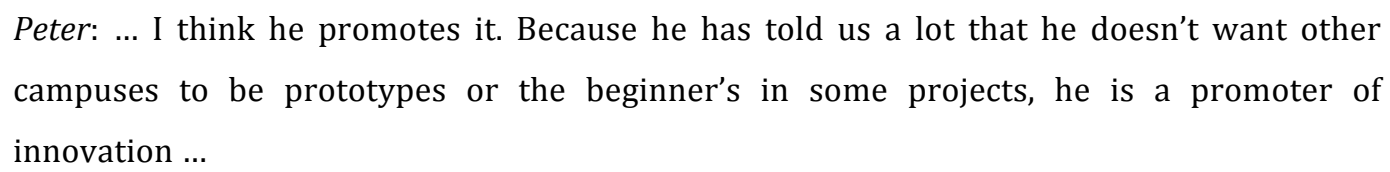

After analyzing deeply more that 70 codes and more than 250 mentions, very important and interesting conclusions arises. To sum up, decision making in ambiguous sceneries is very common nowadays, moreover in high and medium level directors. As Mintzberg (1976) claims, in complex decision making the use of both sides of the brain is imperative, and gut will play an important role (Hayashi, 2001). We could see that expected results arise during the interviews. Most of the interviewed directors are high or medium level risk takers that are not afraid of defending their ideas and innovative projects as long as they deeply believe that are necessary for the differentiation of their division or campus. Several examples and ideas support that not just the risk, but also gut and intuition were mixed in the formula when developing a choice in an important decision. Likewise, most of the directors emphasizes in the importance of being executives, making fast and decided decisions instead of being paralyzed by a non end analysis process. As Saul pertinently said, risk and gut are important when making decisions:

Interviewer: But if you are not sure that everything is going to be fine with that fall, intuition should still matter when making a decision? 
Saul: Yes, you can as long as you have a backup that allows you to have that intuition and take decisions quicker.

Concerning the behavior to mistakes and failures, most directors prefer to regret for some mistaken decision that for the lack of decision. As Peter point out "... I prefer to regret and apologize for something wrong, but never being said to myself what had happened if I had done this or that, but I did not do anything". Not always the rational side will win of will prevail. As Simon (1959, p. 254) claims, "I want to know how people ought behave, not how they do behave". Bosses also play an important role, and concerning to them, most of the directors felt supported.

Innovation and explorative projects are also related to risk and uncertainty. Firms need not just to exploit day to day operation, but also to explore (Schulze, Heinemann, \& Abedin, 2008), and dream the future, and the future will never be a $100 \%$ sure place. Mary associated innovation and disruptive projects with the possibilities of failure. When there is support to risk and gut in decision-making, and when the strategy is about exploring new ideas and tactics, then mistakes could happen, most of the explorative ideas involved a big risk, and there the possibility of failed is not small (Lubatkin, Simsek, Ling, \& Veiga, 2006).

\footnotetext{
Mary: The best for each department. We signed up for an international challenge that was called (Baleo Challenge), about automotive innovation. We enrolled students from different majors, 120 students. VIP. It was a total failure. It was a "pay per view", we made a mistake, the department directors specially from business school which were the ones who pushed for this challenge said "I'm sorry, I was wrong" and more because I was super skeptical, because I saw an inherent risk where you put a team with only leaders is complicated. After that we set up a different strategy, "how we deal with this?" well being coherent. This is an innovation and when you innovate you have risk, that was our validation, didn't work it, fine. We go back to the design, nothing else happens, that is coherence.
}

Finally, each director will make their mind regarding to what extent they consider that risk and uncertainty should play an important role in strategic decisions. Some of them will trust in their gut, some of them will not. To end, I leave two completely 
different responses from two directors on the same situation, the opening of a new career. Here we can appreciate, how risk is playing an important role. The question was whether they would open a new career despite market evaluation and other variables that were not completely in favor, here the contrasting responses, the first one a riskier decision maker and the second more conservative:

Carl: ... of course yes, definitely, and within the same adventures I have had, it is related to gut the big decision of opening a new career some years ago, when marketing research said no, but my gut said yes...

Interviewer: ... and if the number of possible students to enroll in the new career tells you that it seems that you will not have enough to open it, Would you open it even that negative numbers?

Mary: I think I would not. 


\subsection{Analysis of Strategy Theme.}

Strategy is a theme that acts like an umbrella for the other themes that are going to be analyzed. As we saw in the literature review section, strategic management in HEI has taken a relevant position due to the fast changing environment and the increase in competence. There are a lot of definitions about strategy, but it always refer to those actions or tactics that are planned or proposed by the governing board of the management team that conducts the operations to achieve the goals of objectives (Thompson, Peteraf, \& Gamble, 2010) in order to gain a sustainable competitive advantage through the constant search of differentiation (Porter, 1980).

The analysis of strategy theme is composed of 399 mentions which shows the relevance and centrality of this theme within the participants discourse. Table 14 shows the 9 reduced codes (or codes) for the Strategy theme in detail for each interviewee; Table 15 shows the same Table but without the detail per interviewee. Meanwhile, Table 16 shows the extended codes belonging to each code in detail for each interviewee; the codes are indicated on black blackground with white letter, and below each code their correspondent extended codes are shown; Table 17 shows the same Table but without the detail per interviewee. Finally, all codes that were mentioned 4 or more times are indicated with a tight boarder just in the cell where the name of the code appear. This is the same format that is going to be used in the analysis for the rest of the themes.

As it can be seen, Table 14 is composed by 9 reduced codes (codes $A$ to I) that grouped the 399 mentions regarding to strategy theme in such logical way that permits the analysis and addresses the research objective. Some of those codes are emergent codes, like Strategy TEC Negative Issues and Strategy Possible Threats (codes $\mathrm{F}$ and $\mathrm{G}$ in Table 14) that were not planned during the interview, but apparently seems to be very important in the mind of the respondents. It is very interesting to notice that Mike has just two mentions, very few compared to the rest of the respondents, mainly because of the lack of interest and the short responses 
and evasive to the topic, I remember clearly how difficult was to inquire about this topic, and to understand how was the deployment of his strategy. Next we will see that those codes related directly to the purpose of the interview are the ones with the largest percentage.

Taking into account the above and the objectives of the research, the questions of the semi-structured interview were designed in order to inquire mainly in three aspects: how interviewees perceived their strategy from their chair, how they perceived the general strategy of the University and what were the main differentiators in their strategies. Codes Strategy Interviewed, Strategy Interviewed Differentiation and Strategy Planning (codes C, D and E in Table 14) collect all the quotations or mentions where the interviewees commented about their strategy and their differentiations and competitive advantages (Grant, 2010) and together they represent about $56 \%$ of the interviewees, very representative; reduced codes, Strategy Alignment, Strategy TEC Time and Term Strategy TEC (codes A, H and I in same Table) help to respond the question related to how respondents perceived the general strategy and how aligned they claimed to be; I also considered the emergent codes Strategy Negative Issues and Strategy Possible Threats (codes F and G) which have important information about negative feelings and threats for the strategy and that is part of the concerns of interviewees. Now lets analyze deeply what the respondents said about strategy, how that information will be useful to respond the general and the specific objectives. We will not analyze according to the importance or the percentage of reduced code in Table 14, but according to a coherent line that facilitates the final analysis and the cross-reference with the rest of the themes.

Lets start discussing about the importance of alignment to the strategy in the organization and how respondents feel about it. Alignment in all firms is very important for success and achieving objectives (Cohen, 2008) because the strategy deals with leaders trying to guide and establish direction in a team (Mintzberg, 1987). Regarding to this topic, and making reference to the analysis of codes A1 to A3 in Table 14, all of the directors but George showed in some moment of the 
conversation a positive attitude (extended codes A1 and A2) about alignment to the strategy of the University. Next, three extracts from three interviewees are shown; first extract belongs to those directors that were completely convinced and shows a great positive attitude about the "new" strategy, second and third extracts belong to other directors that go beyond expressing a strong alignment with the leader and with specific objectives and KPI:

\begin{abstract}
John: ... if we are doing things well and because we are actually doing it well, it is the time to change and that can shake the whole organization and even generate a negative reaction towards it, and that requires a lot of courage and requires vision and I'm convinced that it (the current strategy) will lead us to a better stage or level as an institution...
\end{abstract}

James: ... "yes, but the organization is moving very clearly and we know our boards KPI's". Then, what's the plan, the vision for Campus San Luis towards 2022 and 2025?. It is an objective in which I have to work along with my leader, as well as the vision we have for the upcoming five years.

Interviewer: And what you proposed as your vision, as a strategy, is it aligned with Tec de Monterrey?

James: Absolutely. As a matter of fact, I have this chart, from being aspirational, from the vision, strategic initiatives, and in which ones I'm focusing... One of the big challenges as a leader in this century and at this time is the focus, because there are 10 thousand projects, but do not have to forget that you always have to pay for them. Prestige, academic quality...

Two directors expressed their concern about those persons that are not in favor of the current strategy, and they focused their answers in training and persuading people as an important part for alignment. Regarding this, Frank said:

Interviewer: Yes, and how you are aligned with your objectives, your goals to the Tec strategy?

Frank: First, every training we have it align us, second, well the experience you already have it as a professor, we have done it already, I mean I've lived every change that it is been done. I'm not sure if you are referring to that but inside the operation on a daily basis I have to be sure that everyone is convinced that this is the strategy that we have to follow. 
Saul even commented that when defining the strategic differentiations, the alignment to the University vision is essential, and even when flexibility is important when defining strategies and tactics, he always maintain an attachment to the vision:

Interviewer: So, you created the vision or you just inherited?

Saul: Oh no no no, this is more of an organizational issue and because in particular we define two things: we have a vision as Tec de Monterrey and that is our duty to be this is the main thing and then we have sort of this like flexibility in each area and in each campus and then we a vocation as campus with clarity of the direction we want to give as a campus, then I said: "Isaac I invite you to tell me clearly what is the vision for this campus, it was very clear: Innovation and entrepreneurship.

We can summarize that almost all respondents showed alignment, in different ways and different intensities. But there were two specific persons who somehow expressed negative comments about alignment (extended code A3 in Table 16). One director, Albert, expressed that different campus were not aligned because processes were different in each campus, and performance and structures were also different among campuses; the other said that he was not able to define differentiators in his division because the university was not taking into account a robust process for excellent professors, specifically this is what the director expressed:

George: We have differentiations as Tec, not as business.

Interviewer: Would you like to create one?

George: I can't but I would like to.

Interviewer: $0 \mathrm{k}$

George: Tec prestige is something very rewarding.

Interviewer: An umbrella?

George: I'll hang from there, I have very good reputation, I have very good relationships with the community, I have very good employability, here's where I am reminded of prestige, infrastructure, but what is essential for a university is the faculty. Many who have come and I have heard say "hey pretty nice facilities" but where are the PhD's? where is the production of knowledge? We are, Alva said, we are a formative university not a research university. In our rankings the main indicator is research, you are evaluating it and that is not fair. 
We saw that alignment is important. Now we will address the main topic in the strategy theme, how interviewees are defining the differentiators for their campus or divisions and how they define their strategy. The questions related to this topic generated such different kind of answers in different levels of profundity. Strategy in HEI takes a great importance nowadays because of the increasing complexity of universities environment and because, in general, strategies in universities are more and more oriented to seeking for revenues and are acting more as business than as academic entities (Washburn, 2005). Also according to Washburn (2005), university presidents are key because of their abilities to raise money and deploy strategies. The extended codes (C1 to C62 and D1 to D21 in Table 16) related to the strategy of the interview are comprised in the reduced codes Strategy Interviewed and Strategy Interviewed Differentiation. As it can be seen in Table 14, about 56\% of the mentions in Strategy Theme correspond to the referred codes with a total of 225 mentions, again this this groundedness - the number of quotations related with each code of theme - reveals the relevance of these elements in the understanding of the strategy theme. All of the directors but Mike, made a lot of comments regarding to their strategy. It is important to mention that in the case of Mike, several questions were evaded, or answered from the context of leadership, but not of the strategy. It is also interesting to highlight that Mike was the person that shows the most risk decision making aversion and also a complete attachment to regulations, it does not matter if that affects some decisions that could have good results; it seems to be an outlier when compared to the other directors, nevertheless in some occasions though few, his mentions and opinions could be relevant and even contrasting.

Analyzing the codes and quotations, according to the most mentioned ones, it can be seen that most of the directors said that their focus was in academic quality (extended codes C4 and C5 of Table 16), prestige of University (extended codes C38 C40 of the same Table), development of excellent faculty (extended codes C41 - C45), the search of a differentiation area that would seize the strengths of the region - it is called vocation of the campus, in Spanish "vocacionamiento" - (extended codes C51 - 
C59 of Table 16) and the development and strength of career's ecosystems (extended codes C16 - C17). Because strategy is not just alignment but also finding sustainable competitive advantages (Burgelman, 1991), the analysis of content is crucial, and that is what we will perform next.

Regarding to academic quality, we can see that it is a principal concern, and even some directors have very clear that it is the first priority, and if they must let down some relevant indicator they will. Extended codes C4 and C5 are directly related to academic quality, with a total of 11 mentions from 6 directors. Nevertheless, it is important to clarify that academic quality is also very near related to other concern in strategies, such as professors, educative model and prestige that will be further analyzed, and then strengthen the importance of the topic of academic quality in the strategy of a University (Dooris, Kelley, \& Trainer, 2002). Regarding academic quality, John is direct when claiming that academic quality is key:

John: ... because I am emphatic when I say that, in this campus, the main strength is academic quality...

Next, Peter claims that attraction of students should be made through a good academic quality, and also claims that having good professors should be a focus on the strategy:

¡Peter: ... and the part of attraction it's done through academic quality, if you have a greater reputation, your ability of attraction it is going to be easier. What we have been doing in this campus and in different campuses in medium cities is to go for the students, be aggressive with students, scholarships, give them freebies that it was pretty common with previous management. Now that has changed, the part of attraction falls down but well, through academic quality, through what we are looking for by having better professors, to encourage research in sort way, encourage by bringing foreign professors, classes in English. 
Two final mentions about academic quality shows how John is locating academic quality above other indicators and how Laura claims that academic quality has always been the distinction of Tecnológico de Monterrey:

Interviewer: Do you have a problem by having a yellow flag in one of your indicators? Does your ego allow it? Don't you want everything in green just to prove something?

John: Not because of the indicator, yes it affects but it is not only my idea, I respect the work of my collaborators and if one of the tells me, hey truth is I don't have anything in order to meet the indicator with a fair argument, let me give this other example, it has nothing to do with KPI's but it is because we can fall into a trap, see we have this football team y our position as a campus is always being good, I use sport as a weapon as a tool for training obviously I want competitive teams but not at any price.

Laura: Look we are always strengthening our academic quality, I think that is something that you can always improve and always looking forward to grow, that's for sure. And I don't do it just now, we've been working on it always because this is what Tec de Monterrey is, is something that identifies us...

As we said, academic quality is also related to the level and quality of professors, and it is interesting that some directors are so aligned to academic quality that even for enrollment of new students, the strategy is related to maintaining excellence in academic quality. Extended codes C41 to C45 in Table 16, are directly related to professors as one of top of mind strategies for the respondents, with a sum of 30 mentions, it reveals the importance of this topic in the strategy of the interviewees. Most of the directors in some moment of the interviewed express the importance of developing an excellent faculty; the cooperation with professors of other campus (seizing the advantage of being part of a multi campus University), the increase in the practical experience of professors and the increase in the number of international visiting professors. John in a very direct way related academic quality and faculty as the main competitive advantages for his position:

Interviewer: ... and which are your main competitive advantages that you have developed in your campus?

John: Academic quality, the strength of the faculty... 
Also regarding to professors, Mary again emphasizes in academic quality and the best professors, local and visitors ones, as a specific sustained competitive advantage; she also comments about seizing the advantage of being a multi campus university, bringing the best professors from other campuses to her own campus. She combines several strategic differentiators that could trigger a good differentiated strategy:

Interviewer: And how easy are these two, so competitors can't copy it?

Mary: Well that is a great question, I believe that depends on how you execute it, I mean, if you can put barriers to entry you give down, for example, I say creative models is (hiring). For example, if I to a student I invest three foreign professors in one semester you say "ok go for it" it is pretty difficult for another university to follow me. What do I have to do here? Coordinate the Tec muscle, as a matter of fact if I want to do it by myself it's impossible, you'll laugh till you die. I have my neighbors who can lend me professors, so if you coordinate this and put it in your portfolio well you say "it's impossible". I think that from the coordination of the different Tec zones will make you stronger for the things that you are not prepared for.

Continuing with professors, several directors argues about two very specific differentiating aspects of professors: on one side, and related to global vision, the importance of a solid faculty that could be complemented not just with local professors, but international ones; and second, professors that have strong practical experience. Those characteristics are directly related to the strategy of Tecnológico de Monterrey University that was explained in Chapter 1, Section 1.1.

The development of ecosystems of career was also broadly mentioned by several directors. An ecosystem of career consists in all activities besides regular classes, were students complements in a practical and sometimes in a playful way, their knowledge. Here the link with practical activities in different industries and firms is essential for students. According to the interviewees, the relevance of ecosystems of career is the quality of experiences that the student could experience during their 
stay in the bachelor degree (extended codes D5 and D6 in Table 16). This is what Albert claims about the importance of ecosystem of career:

Albert: we have problems, for example biotechnology and mechatronics share some stuff, they are consolidated academic programs in terms of student attraction, consolidated in terms of projects, I mean by the end of the strategy the program director that generates experience, interesting projects but I see or feel in both cases a certain element of inattention or that the student, talking about the student from 5th semester and up, they feel that the director focuses more in high school and juniors in terms of attraction.

Meanwhile George comments the following regarding to the relevance of ecosystems of careers and how they represent the base for his strategy:

George: We are moving to ecosystems. We want 20 careers 5 ecosystems. We are working on the business and humanities ecosystems, these are the ones that I manage. Engineering has other ones.

We are migrating to the business area, as a matter of fact, the program director from Marketing and communication (LMC) when he sells the program or bachelor he also promotes the Paths program too. When the program director form finances sells his program he also speaks about the Path program. When it is time to promote the different programs, not all the program directors have to go, because they talk about business.

I'm not measuring them by their student attraction to the program but because on how they work with their ecosystem in business, what I mentioned once to the committee was that they should evaluate student attraction at zone level not for each campus because this avoids campus collaboration. How about if you say "hey no one wants to come here" but I have contacts and I can share them with you, they tell to RECTORIA and we will be awarded, if working as a team it's better. So what you say about program differentiators its more because of the business school, it is by the ecosystem.

One of the most difficult part of the strategy consists in finding competitive advantage, "the job of the strategist is to understand and cope competition" (Porter M. E., 2008), and besides academic quality and professors that were already mentioned, directors also pointed out to other actions or tactics in their strategies, such as innovation in the educative model. As we already saw, Tecnológico de 
Monterrey is immersed in a new educative model, where innovation and technology in the class is very important. This topic was also reflected in the opinion of the respondents, with a total of 15 mention from 6 directors, all of them division directors. Laura mentioned several innovative initiatives, such iWeek, iSemester, Vertical Workshop, etc., that integrates this new model called Modelo TEC21, that comprises educative innovation, and which stakeholders and confirmatory interviewees confirmed as a fundamental part of Tecnológico de Monterrey current strategy. This is what Laura pointed out:

Laura: Since last year, we started to work in a project that it seemed very interesting, we did vertical workshops for all the 700 students from business school, where all the professors were involved, all program directors and all department directors. All school personnel were involved in this and for me that was very important because that sums up to Model Tec21, and developed abilities and we even detected some areas of opportunity so we can work aligned to Model Tec21 and work correctly for what is coming for Modelo Tec21, for example for iSemester design and some of the activities for Innovation the iWeek. We ran this vertical workshop in April, last April and we had 5 vertical workshops, from those 5 workshops students were distributed, we had teams of professors coordinating the students, they were the leaders of the challenges, we've never done anything like that, all the time leaders were the department directors or program directors. No we changed the roles, professors were the leaders.

According to Clark, one of the confirmatory interviews and the one with the highest position, another important strategy for each campus consists in searching areas of expertise and differentiation according to the strengths of the region (the word in Spanish is "vocacionamiento" and will be used this way in the rest of the thesis). In codes C51 o C59 of Table 16 we can see that 4 directors claims as an important part of the strategy to focus on finding this expertise area of differentiation ("vocacionamiento"). Specifically, several directors mentioned different areas for their "vocacionamiento", such as: automotive industry, entrepreneurship, innovation, manufacturing and mechatronics industry. Regarding to this, Carl expressed how important is vocacionamiento for one of the campus: 
Carl: ... then we have to increase the population in bachelor degree and with clear and concise "vocacionamiento", seizing the advantages of the region we will be able to specialize in something and not diversifying in a lot of options that could jeopardize the strategy...

Another part of the mission of the University is related with global vision of the graduated students. Related to this three directors mention that internationalization and global vision of students was key for the differentiation (extended code C28 in Table 16). Next Peter's comment:

\begin{abstract}
Peter: ... promote to bring foreign professors, promote courses in English, that also encouraging to have classes in English it's been a huge shock because we have students coming from other high schools and you have to double the mix, I mean we get criticized that we offer Spanglish, because you have to explain in English and then comes this guy from other high school, because has zero knowledge of the language but he's already in and he needs to adapt, so I believe if we are there in the international part, this campus has more than $75 \%$ of people that graduates with international experience and obviously the tendency is to grow to reach a $100 \%$.
\end{abstract}

Summarizing according to the extended codes $\mathrm{C} 1$ to $\mathrm{C} 62$ in Table 16 belonging to code Strategy Interviewed we saw that academic quality, faculty, ecosystems of career, "vocacionamiento" and global vision, were the main concerns in the mind of the interviewees regarding their strategies (they represented the most mentioned codes). The rest of the extended codes for reduced code Strategy Interviewed received a few mentions, just one or two, meaning that they did not represent enough groundedness or strength such the above ones did. It was very interesting to notice, that almost none of the directors, but one, mentioned that his strategy was based in facilities. It is also very important to highlight that three of the directors claim that this strategy focus was not reflected in the indicators that are negotiated with their boss for the work plan of the year (extended code C48 in Table 16). They mention that this explorative initiatives that could provide sustainable competitive advantage, generally are not written in that objectives plan, because it is mainly composed by exploitative KPI. Also interesting to point out is that the quality of service and seizing the advantages of being a multi-campus university were not in 
the list of the most mentioned strategies, in fact, there was just one mention for each. Maybe it reflects the fact that 4 directors said that there was not one University strategy or any unified processes in this multi-campus university (extended codes I13 and I22 of Table 16).

Finally, it is important to remark that when asking directly to the interviewees about their differentiators in their positions, what we found is that the were mainly focus in the next differentiators: academic quality, professors, prestige of the university, internationalization of students and development of ecosystems of careers (see the most mentioned extended codes, D1 - D21, in Strategy Interviewed Differentiation reduced code in Table 14). Differentiators con provide sustained competitive advantage; Universities must seek those differentiators in a creative way, trying to avoid copying the currently existing ones (Spendlove, 2007). Those topics were almost the same topics that were the most mentioned ones when analyzed what the interviewees claim about their strategy; professors, academic quality, global vision and ecosystem of careers, and it will not add any value and could be repetitive mentioning them again. It seems that all of those initiatives contribute directly to the strategy of the University, nevertheless, it is worth pointing out that no one mentioned specifically those differentiations that the University has claimed, and which were mentioned in Chapter 1, Section1.1.

Up to now, we went deeply in the strategies and the differentiators of the interviewees. Also, we could see that most of them claimed to be aligned to the general strategy of the University. Now, it will be also important to inquire about the perception of interviewees regarding the strategy of the University. All comments and mentions regarding the general strategy, were joined in the reduced codes Strategy TEC, Strategy Possible Threats and Strategy TEC Negative Issues (codes I, G and F respectively in Table 14). Strategy TEC reduced code and its extended codes, I1 to I28 in Table 16, contain the information regarding to how directors perceived the strategy of the University within a total of 62 mentions. All the directors but Mike, 
mentioned something related to this code. Lets go deeply on the analysis about the perception of the University strategy since the point of view of the respondents.

Analyzing the most mentioned codes and not necessarily following the order of the most mentioned to the less one, first we can notice that 4 directors claim that strategy has a clear and simple formulation, Campus Directors Carl and James and Division Directors Albert and Saul. They claim that the strategy is clearly established as well as the vision and mission, and that the strategy is consistent according to the basic theory of strategy. Regarding to this, and comparing to past strategies Saul claims the next:

Saul: ... in general I think strategy is good, there's a clear short vision that everyone understands, I believe that simplicity gives us a lot of clarity, compared to the past, that were pretty much elaborated visions, missions and strategies with a lot of complex tasks that we had to do, I think this simplicity that has given the governing body are all understood very easy, right ? by saying let's focus on developing entrepreneurs leaders internationally competitive with s human sensitivity

Half of the directors interviewed had in some moment of the interview a positive attitude to the change and to the strategy (codes I24 and I25 in Table 16). Also very interesting was the fact that during the interview some directors mentioned that there was definitely the necessity of a new strategy, like the one that has been introduced in the process of transformation. Regarding this, John points out that the change was pushed partially by a new and complete different profile of the student; in the same line, James and Albert claim that this new strategy and change was necessary because of the necessity of a standardized process among campuses that are not aligned and hence are unable to seize the advantages of a multi-campus university; and finally John and George believe that the change was necessary because HEI are part of a rapid changing world and environment, coinciding with Peters (2005), who claims that in these rapid changing times, strategies are obsolete in a short period of time. John commented regarding to this necessity, and he also related it to academic quality improvement as a strategy: 
John: I believe it is one of those things that you cannot set aside, from my point of view, as an education institution you have to go for it, just by looking what is happening every day not only in our country but in Latin America in the whole world even to say, hey as an academic institution we have a huge responsibility you can't get away from, anyone who closes their eyes to that, the only thing is doing is the easy part of education, the simple part.

That is has nothing to do with training and we are trainers. So, of course we don't have to look away this disciplinary part and that's the challenge, reaching balance between graduating good engineers, competitive enough internationally in their discipline but with human sensibility, which are related and requires conscious development in different learning areas.

Regarding to how interviewees perceived the strategy we can see in extended codes I5 to I15 and I20 to I23 and I28, that there are so many different answers, but the most repeated ones are related to two different and specific issues: on one side the strategy focused in creating a new educative model (codes I12, I14 and I23 in Table 16) and on the other hand, the strategy of the University focused in improving academic quality, reputation and becoming students competitive worldwide (codes I20 to I23 and I28). Next Frank points out the new model as one of the main goals of the global strategy in the University, and at the same time it is very interesting that some possible threats and risk related to an high cost model arises. Further in this theme analysis we will see those risks and threats that the interviewees are considering in their minds.

Interviewer: And how do you perceive from Salvador Alva's point of view, from the top, the strategy that Tec has, how you perceive it for yourself, what's the strategy that Tec de Monterrey has and how far can you see it?

Frank: I honestly think, that strategies are good. I see an education model, I love this education model, what is this paths doing, I see it very good: about research I think it seems the only thing we can do, things that I don't precisely like, that I think all this model is too expensive and it is not going to be worth it.

On the same line, Mary also relates the strategy to a new educative model, and emphasizes in quality of service: 
Interviewer: And what about Tec de Monterrey strategy? How you perceive this strategy in this moment? What is Tec de Monterrey currently doing?

Mary: It is an interesting question; I see it like this two lines: maybe I'm wrong but it's my personal opinion. I see an academic line where we want to give, we want to prepare these kids for the next level, see this part of educational innovation, flexible education, and that's what excites me and moves me. On the other side I see a Tec based on service.

Some directors made opinions related to the focus of their strategy in addressing specific indicators such as employability or internationalization (extended codes I10 and I11 in Table 16). A few strengthen the idea of the strategy as the mean for standardizing process in all campus (extended code I13). Despite dispersion in those perceptions, after the analysis it is clear that most of the interviewed are considering the University strategy as focused in the development of a new educative model, based in the use of technology and at the same time focused in prepare the students for living and succeeding in worldwide competitive sceneries.

During the interviews it was clear that when referring to the topic of University strategy, several negative comments and concerns related to the strategy started to appear, all those comments were joined in the reduced code Strategy TEC Negative Issues (see Table 14). But not just negative issues, also several comments related to threats that could jeopardize the strategy came up, those comments belongs to the reduced code Strategy Possible Threats in Table 16. Those codes arose without planned scenery, as they are emergent codes (Boyatzis, 1998) As it can be seen in Table 14, both codes represents about $13 \%$ of the conversations, that number is not negligible.

Regarding those negative comments about this new strategy that started about 4 years ago, we can see in extended codes G1 to G18 of Table 16 that most of the directors, 8 in total, formulated opinions to this concern, that is specially relevant because there were not questions during the interview for this specific topic. Especially, George and Albert, both division directors, had the most negative view 
about the strategy with a total of 8 mentions each. In general, the most mentioned concerns were: the excessive centralized and complex processes mentioned by 4 directors (extended codes F10 and F11 in Table 16); lack of planning and failure in implementation, mentioned by 4 directors (extended codes F5 and F8 in Table 16) and lack of communication and alignment, mentioned also by 4 directors (extended codes F7 and F9 in Table 16). Frank and Mary exposed an interesting comment about how innovation could convert into a negative thing, if the decision makers are not aware that all innovative actions are deployed with the enough academic quality (see extended codes F3 and F4). Regarding those negative comments, that were the most mentioned, important extracts of the interview are added in order to exemplify in a better way the context of those comments. The first extract refers to the lack of communication; the second make reference to a good intention but a failed implementation; and the third one is also very interesting negative issue and exemplifies what James calls strategic insensitivity, in the way that they are trying to copy that strategy from the industry.

Carl: No, it is one of the issues that we are dealing with right now, interfaces are not working in their best possible way. Even though there's an intention that it should be like that, if you go and ask program or department directors, even professors, It wouldn't be strange to be confused, I mean that we don't know exactly where we are going.

George: Overall, I feel that it's been a great change, an aggressive one, changes that are happening in the education industry I perceive them as radical. It is impressive what we are going to deal with in the upcoming 5 years and I think we are making this changes on time. The intention is good, the implementation leaves too much to be desired and implementation I see it from my level, probably from the top management level they don't see things as I see it. What I see, what I have to deal with or enjoy, I see they are a lot of areas of opportunity; implementation is not on its best shape.

James: I think that in some point, the structural corporate process is fine but when its time to download it to an operational level I feel this strategic insensibility to understand what is really happening in our city or campus. Leaderships are fine, people that has come is actually good, but still sensitive, it has cost them a lot of work to understand that we are a university and not a production line. 
But not just negative issues came up; also threats for the current strategy were also identified (extended codes G1 to G18). James and Albert were the persons that predicted more threats for the strategy. In general there are several different threats that arose, and analyzing codes there were not specific ones that had much more mentions than others, in fact, all of them had 1 or 2 mentions. The arriving of international competence (USA universities specifically) and the fact that Mexicans decided most of the times foreign companies instead of local one was mentioned by 2 directors, George and Albert, as a big threat (extended codes G6 and G7). Also very interesting was what Laura, Saul and Mary, said about the negative side of the initiative of the homologation and centralization of processes as part of the current President's strategy; they complained of the slow and very complex processes that inhibited the innovative and explorative side of directors (March, 1991). Following, very illustrative what Saul commented about that:

Saul: I believe that in terms of areas of expertise, in returning all decisions to Monterrey being a monster, helps I mean makes decision process slower, so we say we want this fast yes, but the decision making process has to at least go through 10 people so they can say yes or no, let's go back. Back then processes were much simpler from a campus or region, those decision making processes were faster and now I feel they are making them slower, so the approach that was intended with this vision that was going to be achieved in a given time, I think we will have to extend it a bit if things remain like this.

Continuing with those negative issues, George and Frank, mentioned that the new University strategy will demand a significant increase in the percentage of full time professors, and the hiring of many research professors, turning it into a very expensive model that would be infeasible for the campus. The difficulty in changing the culture and the inertia to change would represent two more threats mentioned also by 2 directors (extended codes G2 and G3 in Table 16). Peter, George and Albert, pointed out as a big threat the fact that there were a lot of changes in parallel, and the day to day operation - exploitation - could jeopardize the long term goals of the 
strategy - exploration - (see extended codes G1, G16 and G17), this is how George expressed that:

George: Timing is good because we can survive the future, but probably what my complain is, is the way the execution is done, because drastic, fast and numerous changes are not good at all. Drastic, quick and a lot of them can really be a threat.

Let us make important conclusions of this theme analysis. Now we already analyzed those codes that provide rich information for the strategy theme, both from the perspective of director's own strategy in their positions or from the perspective of the University strategy. Regarding to the interviewees strategies, we realized that most of them are well focused in a bunch of initiatives related mainly to: improving academic quality; the constant improvement of ecosystems of career to encourage the experience of students; the strengthening of a local and international faculty; and the strength of a global vision for students. It is also rewarding to see that in general there is a tendency to the acceptance and alignment to the strategy of the university; most of the respondents felt positive, and also reflected that in their own actions and tactics, whether they were division directors or campus directors. It is important to be aligned when deploying the strategy, in this context Tierney (1988) points out the importance of being aware that due to differences in mission and objectives different HEI can perform differently, and then bring negatives issues, and then alignment is important.

As we already pointed out, HEI are facing rapid changes, product of a constant concern because of the complex environment and rapid changes in the environment (Yielder \& Codling, 2004); (Timberlake, 2004); (Tavernier, 2005), and it seems that interviewed directors are aware of this situation, and they try to innovate and bring differentiators to their strategies in order to create sustained competitive advantages (Porter, 1980), and this way, according to the analysis of each interview the strategy of each director seems to agree to the idea of Kaplan and Norton (2004), who support that every company's strategy describes its intentions to create value for 
their shareholders, customers and citizens. Going deeply and trying to see those little important details in strategy, I would like to mention that 4 directors talked about the importance of trying not to copy but adapt those strategies that worked in other campuses. As Tierney (1988) pointed out "institutions with very similar missions and curricula can perform quite differently because of the way their identities are communicated to internal and external constituents and because of the varying perceptions these groups may hold". Regarding this it is interesting to see how those 4 mentioned directors were sensitive to the fact that not always copying a strategy just for copying it will bring the same and desired results that happened in other campuses or companies, this is how Albert said that:

Albert: .... In Guadalajara this Works perfectly so I copy the strategy, then when I get there he starts having these problems let's say, why is this here, hey that thing you call 360 we did it 5 years ago and it is not working, hey what is that, we've done that already and didn't work, so in some way I say, with just a bit of premeditation or learning from others, I tried to avoid that situation.

Finalizing, I want to remark that when deploying the strategy, alignment and communication are important, but also execution and the self confidence that things work well even in uncertainty and difficult times, that self confidence that will allow the strategist to defy status quo and defy the chief believes; self confidence that will diminish the avoidance to take risk and to fail under ambiguity and complex sceneries (March \& Cohen, 1974). Regarding that, this is what John said during his interview:

John: I receive my objectives from my boss and I'm aligned to them, my boss aligns with his boss's then in that objectives waterfall it doesn't mean that I do all of them, in fact there are some of them that I tell my boss, well this one I don't get it I know you wrote it down but let me tell you that even in my dreams or not, not because is unreachable or we are not doing a good work but because I also don't believe let me explain, for me again you have to argue intelligently why yes or not, so you tell me "hey there's a KPI about internationalization for high school that we want to reach a figure by 2020, and I always wonder at what expense? Not economically, at what expense? It means I have to reach that number? If I am not able to do 
well, in other words, If I jump by risking my community in order to meet and indicator only, being young teenagers and then they're loose around because I don't have the capacity to join them with professors because the program he's doing, from my point of view has nothing to do from our point of view the necessary supervision. 


\subsection{Analysis of Ambidexterity Theme.}

Now we will analyze the theme regarding to ambidexterity. Fast changing environments demand the firms a correct balance between those activities related to exploit the day to day operation short term activities in an efficient way while at the same time exploring those new ideas and possibilities that will become the competitive advantages in the long term (March, 1991). According to the above objectives, a total of 10 codes (remember that when referring to codes it means reduced codes) were detected, which arose from the reduce process of the extended explained in Chapter 3, Section 3.3.5. The analysis of Ambidexterity theme is composed of 281 mentions which shows the relevance and centrality of this theme within the participants discourse. Table 18 shows the 10 reduced codes (or codes) for the Ambidexterity theme in detail for each interviewee; Table 19 shows the same Table but without the detail per interviewee. Meanwhile, Table 20 shows the extended codes belonging to each code in detail for each interviewee; the codes are indicated on black blackground with white letter, and below each code their correspondent extended codes are shown; Table 21 shows the same Table but without the detail per interviewee. Finally, all codes that were mentioned 4 or more times are indicated with a tight boarder just in the cell where the name of the code appear.

Table 18 also shows the percentage of mentions (or quotations) that belongs to each reduced codes. This percentage is directly related to the importance of the topics for this dissertation, then the biggest percentages belongs to how ambidexterity, exploration and exploitation is deployed, the types of ambidexterity and how ambidextrous are interviewed, their bosses and the teams. Next these main topics will be described and related to the reduced codes in the next paragraph.

The main topics that were intended to address during the interview regarding to the topic of ambidexterity are: the level of ambidexterity of the interviewees, their bosses and their teams (related to codes Ambidexterity Interviewed, Ambidexterity 
Team and Ambidexterity Boss in Table 18); the ways interviewers deployed ambidexterity with themselves and with their teams (related to codes Ambidexterity How happens and Ambidexterity Team in Table 18); the kind of ambidexterity that is deployed whether interviewers deployed (related to codes Ambidexterity Contextual and Ambidexterity Structural) structural of functional ambidexterity intentionally or unconsciously (Raisch \& Birkinshaw, 2008); the way high level directors should behave related to ambidexterity (related to codes Ambidexterity Successor and Ambidexterity According to Chart in Table 18); special topics related to exploitation or exploration (related to reduced codes Ambidexterity Exploration and Ambidexterity Exploitation); and finally, the relationship between ambidexterity and the strategy and decision making is going to be extracted not from direct questions but from the context and the answers of the questions related to this topic; for this topic Ambidexterity KPI reduced code is important, because it is related to those short and long term indicators according to the point of view of the interviewed. Lets analyze the quotations related to the different topics in detailed in the next paragraphs.

Regarding to the level of ambidexterity, there were two differentiated responses here, one regarding to how good are they for exploitation or exploration; and the second regarding to how much working time is distributed among exploration and exploitation. The responses related to the level of ambidexterity of the interviewees are presented in code Ambidexterity Interviewed (code A of Table 18 which is integrated by the extended codes A1 to A13 in Table 20). Analyzing extended codes A5 to A7, it is shown that several of the interviewees, 7 to be precise, claim that in the day to day operation they are more dedicated to exploitative activities than to explorative ones; those directors dedicate $30 \%$ or less time to exploration, and $70 \%$ or more to exploitation: 5 from those 7 dedicate less than $20 \%$ of their time to exploration; in one case, referring to Saul, he claims to dedicate more than $90 \%$ to exploitation because he has less than 1 year in the position and he is forced to spend most of its time operating, nevertheless he has a good sense of strategy and 
competitive advantage as it could be seen in strategy analysis in Section 4.2. In order to illustrate this situation, three selected exstracts of the interviews are shown next:

Interviewer: What is the percentage of your time that you occupy in one and in the other (referring to exploitation and exploration)?

Carl: If I talk of a specific year, for example 2015, I could tell that about $70-80 \%$ is exploitative and approximately $20 \%$ percent explorative.

Interviewer: So roughly, what percentage of the day to day operation (exploitation) and what percentage of the other (exploration)?

Laura: I would say $70-80 \%$ day to day, which means $80 \%$ to operate and $20 \%$ to do the rest (when referring to explorative activities).

Interviewer: Do you consider yourself a person who runs more into the short term or towards the long term or a balanced person?

George: Balanced to long-term.

Despite the fact that most of the directors spend significantly more time in exploitation activities; it is interesting to show a very important contrast, it is that they consider themselves more attached to exploration than to exploitation activities, then the reality differs from the conception of them selves. Analyzing extended codes A2, A4, A8, A11 and A13 in Table 20, all of them points out that directors in general seems to be good and even desire to work more in mid to longterm activities (those belonging to exploration). John makes a very interesting point when commenting that even though he just dedicated $30 \%$ of the time for exploration, this 30\% seems to generate great results, above the results that generate the rest of the $70 \%$ that was dedicated to exploitation, this means exploration was privileged. John and Joe comment the following regarding to exploration activities:

John: ... I think I'm privileged, even that Tec nowadays demands from you a lot of exploitation, even for the director of the campus, and that is my complain because you pay me for another things, I'm privileged because thanks to my team I could have more time to explore, I couldn't do that without this great team. 
Interviewer: Are you more focused on short, medium or long term? Or how do you consider yourself in that part?

Joe: I consider myself as long term. I'm a dreamer; I like to see how things could be in the future, not as they are.

Mike answered in a different way regarding to the level of ambidexterity, claiming that he behaves in a dynamic way regarding to the allocation of time for exploration and exploitation activities (extended code A3 in Table 20):

Mike: I don't always go with the same scheme, talking about how much time I am going to dedicate to each thing, I mean, if I need right now to dedicate $100 \%$ percent to operation I will, if I need a little time for strategy then I'll give that time to strategy, and so on.

In the context of dynamism, it is very interesting to point out that one of the directors, Albert, commented that the time dedicated to exploitation versus exploration changed in the two years that ha had been in this position. While the first year was $100 \%$ exploitation, the second year was just $80 \%$ exploitation and $20 \%$ exploration. This result is more related to a functional or contextual than structural way of doing ambidexterity (Schulze, Heinemann , \& Abedin, BALANCING EXPLOITATION AND EXPLORATION Organizational Antecedents and Performance Effects of Ambidexterity, 2008).

Now lets inquire about how interviewees considered that ambidextrous behavior should be in those directors that will occupy their positions and those in higher positions. The codes Ambidexterity Successor and Ambidexterity High Directors are related to this topic (codes B and C in Table 18 and its respective extended codes B1 to B9 and C1 to C9 in Table 20). This analysis is important because approaches in exploration or exploitation require different structures, vision, strategies and context (Raisch \& Birkinshaw, 2008). First we will analyze code B, it means interviewees opinions about the balance among exploration and exploitation that they consider should have the persons that will be their successors in the future. Should they be more explorative, exploitative or should they be more balanced? Regarding to ambidexterity of successors (interviewees were asked how ambidextrous should 
their successors be) 6 from 12 directors made some relevant opinion on the topic. Three from those 6 directors claimed that their successors must be somehow equilibrated (extended codes B3, B5 and B6 in Table 20); it means they should be good in short-term operation (exploitation) and good in long term (exploration). They claim that, in general, their successors should be very good in exploitation activities, that they should be equilibrated or more focused to exploitation, and even one of the directors in favor of equilibrium strength on the importance of exploitation activities, referring to those that will bring healthy finance to the campuses. Regarding this, next let see the comments of James and Mary regarding the relevance of that equilibrium among exploration and exploitation:

James: He is more balanced (referring to one of his colleagues). He is the one that has the ability to think despite his strict training, he is the one that has the ability to be looking beyond without losing sight of the operation, and that is important ...

Mary: Is a guy who coordinates areas very well, he's cautious? It is not a guy who will breakdown he likes to know new things and experiment but also he know that he has to work on his own duties (operation). I believe that him and I have understood each other very well, he has understood everything and he's doing it all right. I like also how he manages conflict he knows where he is strong or weak and then he tells me "Hey I'm in", so I work pretty good with him.

Another two campus directors, John and Carl, seems to believe that when selecting their successors, the exploitation abilities are more important than explorative ones (extended codes B1 and B8). This is what John claims about that:

John: I'd believe that a balance in that model you mentioned, ambidextrous, I'd imagine $70 \%$ in operation and $30 \%$ in exploration would be very healthy. I mean, if I'm dedicating a third of my life in exploration I need two thirds of my life to put it in practice, evaluate it and have feedback. A 50\%-50\% I would not imagine it because you'll generate a lot of information that you won't be able to put in in practice. 
Although some of the directors assigned high importance to short-term operation in their positions, they considered that it is more difficult to find exploratory persons, an interesting contradiction, as John points out:

Interviewer: from your point of view, what is more difficult to find, people that knows well how to exploit or people that can explore well? and I know it is a very open question but...

John: ... certainly, it is more difficult to find people that knows how to explore well...

Now lets see what directors think about ambidexterity and the balance among exploitation versus exploration in high-level positions. In this case, directors will not make reference to their own positions, but to higher ones, those above them. The analysis corresponds to code Ambidexterity High Directors (code C in Table 18 and its extended codes C1 to C9 in Table 20). All directors but Mike made relevant comments to the topic. This topic is important because according to Gibson and Birkinshaw (2004) the role played by leaders of high directors is crucial for the deployment of ambidexterity, whether the structure and functions of the firm will be more directed to exploration or exploitation or if a balance is the aim. According to the analysis of the reduced code Ambidexterity High Directors and their extended codes C1 - C9 in Table 20, we can conclude briefly that there is a tendency towards exploration more than exploitation in the relevance of high level directors. Summing the number of mentions, we can see that 20 mentions from 34 of this topic, belongs to those directors that in some moment thought that exploration was more important than exploitation in high level directors. Regarding this, Carl claimed that:

Carl: ... as an University, Tec de Monterrey cannot be operated as a manufacturing company that just produce thousand of items. A lot of people think that when they hear "Campus Director" is the same as a "Plant Manager" in other type of business, but in reality it is not like this, because a plant manager is $100 \%$ operative. Here in Tec, we have to find competitive advantages; we have to adjust our reality to our region. Also we have to think, to think and to propose new things, so speaking about Tec de Monterrey as a private university, in the high directive positions, it does has to exist a lot of exploration. 
Following with Carl, and in a very forceful way:

Interviewer: Okay, Carl. Can I infer from you that perhaps in high level positions, the explorative part of the director should be a "must"? And perhaps it will be more desired in higher than in lower positions?

Carl: Definitely yes. Totally agree, are we talking about the university context?

James talk about the importance in high level directors in Tecnológico de Monterrey, in a context where he complains about an specific director who lacks from explorative skills, and at the same time talks about the lack of leaders in the University:

James: ... that is, you choose a completelly exploitative person to lead a new and innovative educative model... then how are we going to achieve that innovation? If at the end I am invited to think outside the box and in the reality, I will be inside of another square box, (when referring to the leader who is far from being innovative), then we're screwed. That is the issue, nowadays in the institution we have more exploitative leaders, I think we need to have more explorative leaders ...

George on his own, claims that lower positions are more exploitative and higher positions are more explorative:

George: Look, day to day operation is unavoidale, but it should not dominate my role. The lower your positions in the chart the more operational you must be ...

We saw that more than half of the mentions are related to the importance of exploration in high-level positions. Nevertheless, despite 10 of the directors in 18 mentions commented that explorative skills and behavior were more desired than exploitative ones, we can not have a general consensus regarding that directors should be completely explorative in those positions. Analyzing again Table 20, we can realize that 6 directors, 3 campus directors and 3 division directors, pointed out a relevance towards exploitation, saying that high level directors should have a balance among exploration and exploitation or even that they should be more 
exploitative than explorative directors; in fact the most mention codes are related to balance in exploration (extended codes C2 and C5 in Table 20) or related to the relevance of exploitation (extended code C6 and C9 in the mentioned Table). Carl points out the relevance of operation (exploitation) even in very high positions:

Carl: ... in those levels in which operation is not very common, I do not doubt that is all backwards, 30\% exploitation and 70\% exploration. But it could be also that a 50\% - 50\%, because at the end they have to handled a lot of aspects of operation in a strategic level, but they will need to operate...

Mary points out that most indicators in high-level position in the University are more exploitative than explorative:

Interviewer: ... in high positions, like the one you have and higher, Campus Director, Rector, etc. which would be the more adequate combination, more explorative or more exploitative? In your opinion, how should be the combination for those high positions?

Mary: That is very interesting, in an utopia, it would be very nice to coexist, but that is not measured by Tec, you see that data in indicators and, at the end of the day, most of them are short term (exploitative) indicators, the sales, the evaluation, etc. and again it is all about cost effectiveness, where the explorative question does not have specific indicators.

One of the directors claim that campus director and division directors are more like "managers" than "directors" referring that they are more directed to exploitation. Lets see the opinion of Albert:

Albert: ... in these positions they are like managers (when referring to campus directors), where $70 \%$ or $80 \%$ have to be operative and $20 \%$ have to be explorative ...

According to some interviewees, those skills that favor exploration or exploitation could be learned through the years and they can change the percentage of time devoted to one or the other:

Interviewer: How do you manage that? If you have to assign 7 points, how would you 
distribute them, how many to your explorative part and how many to the exploitative one? James: 4-3 more exploitative. I think at the beginning I was much more exploitative 6-1, then like 5-2. At first, you have to attend operation and handle it...

In sum, from the above analysis we can see that despite interviewees are more confortable working with exploration, they have a tendency to think about and assure exploitation skills when thinking about their future successors. Regarding to high level positions, we could see that in general it is considered that high level directors should be more focused to exploration than exploitation. A very interesting point of vie that shows some differences in the way interviewees perceived the relevance of ambidexterity in their positions in relation to higher ones.

We can see, even within the same interviewees, different perceptions regarding to how much time they should focused in explorative activities depending on the positions. When referring to their positions, they think that exploitation is very important, and when referring to higher positions, several of them agree that exploration is the key. Here the opinion of Albert:

Albert: I will repeat, I mean, my industrial engineering side is going to speak again. I think we have to move on towards processes, I think these positions have to be managers with 70/80 \% operative, and $20 \%$ explorative.

Interviewer: High level directors?

Albert: I see a difference right there, they should be more explorative.

Interviewer: You see a difference...

Changing to another topic within the Ambidexterity theme, we will analyze how interviewees promote the development of ambidextrous behavior for themselves and within their teams, and in a special way how they promote exploration, that it seems that their teams lack it more than exploitation. This is one of the most important topics for the thesis research, but also for the interviewees, as it can be seen in the percentage of the mentions that received in the reduced code 
Ambidexterity How Happens (code E in Table 18 and its extended codes E1 to E17 in Table 20). Next lets analyze in detailed.

All of the interviewees made opinions about how to achieve a balance in ambidexterity and how exploration should be promoted or incentivized. Most of the interviewed commented about the demanding time of exploitation activities, and the desire to have more time for exploration (as we saw in the above analysis) and at the same time they stressed that the nature of most KPI (Key Performance Indicators) were more exploitative than explorative. Analyzing extended codes E1 to E5, we can realize that the main answers that responses how to achieve a balance between short-term day to day operation - exploitation - and long-term operation exploration -, are focused in finding time for both kind of activities and in a good operational team for delegation. For this specific question about how to achieve a balance in ambidexterity, 5 interviewed directors claim that they constantly switch among explorative and exploitative activities, depending on the demanding of different periods of time and the demanding of the KPI (extended codes E2 and E4 in Table 20). This seems to be more a contextual ambidexterity than a structural one (Raisch \& Birkinshaw, 2008), as we see further in this section. Regarding to this constant change of activities on demand, Mike and Carl said this:

Interviewer: The percentage of time that you dedicate to each operation from your position as campus director.

Mike: Flexible, I can't decide, $40 \%$ strategy and $60 \%$ operation, or $80 \%$ operation. I change proportions on demand.

Interviewer: According to what?

Mike: According to the daily needs and the environment.

Interviewer: Ok very interesting.

Mike: I don't always go with the same scheme, talking about how much time I am going to dedicate to each thing, I mean, if I need right now to dedicate $100 \%$ percent to operation I will, if I need a little time for strategy I’ll give that time to strategy, and so on.

Interviewer: What percentage of your time and effort do you occupy in one position and another? 
Carol: It is very circumstantial and very contextual; there may be some weeks and months that I need to be very operational, very exploitative because sometimes you have to solve fires you have to intervene ...

John, Albert and Saul directors commented that organization and strategic planning while defining the indicators and the way to address them is very important in the correct balance of explorative and exploitative activities (extended code E5 in Table 20). Lets see what John said about it:

John: ... I'm privileged because thanks to my team, I could have more time to explore, I couldn't do that without this great team.

Finally three directors believe that trusting their team in order to delegate, is key for achieving ambidexterity (James, Peter and Saul in extended codes E1 and E3 in Table 20). One of these directors said that he delegated most exploitative activities in order to have time for exploration:

Peter: Well, delegation is important, I mean, if you want to do everything it's complicated. You must delegate some things to the academic director, some others to the program directors; you have to push a little from our directive chair so people actually do things. And sometimes you have to dig in a little with them.

Interviewer: How can you be sure that things are going to happen?

Saul: You have to trust your team.

Interviewees also commented what actions or rituals they have in order to promote exploration in their team. Exploration was the topic, because one way or another, it seems that exploitation was not the problem because persons seemed to be good enough or it was easy to improve exploitative skills (planning, organizing, delegating, etc.). Nevertheless, exploration is more about predicting the future, reading opportunities and setting the path, and then more difficult to achieve (Gibson \& Birkinshaw, 2004). Analyzing the extended codes E7 - E17 in Table 20, there are several important findings. All of the interviewees but Mike made relevant 
contributions to the topic. According to the directors there are several different ways on how they promote exploration. Analyzing the most mention codes, in extended code E10, with a total of 20 mentions across 9 directors, they claimed that they promote exploration in their teams by investing time talking with them about future actions to differentiate in the future, motivating them to look ahead, to innovate, and inviting them to explore new ideas. In fact, some of those directors ask their team members to step down those activities to their respective teams, in order to promote exploration in lower level of the chart. Lets see what John and Carl said regarding the way to promote exploration in their teams:

Interviewer: And what do you do to keep the same rhythm, because it is easy that daily operations don't let you see the future?

John: That is the hardest side of my work, because on one hand I push this to make it happen accomplishing all the short term things and that's operation. For doing exploration, I look for moments, look for spaces, in order to take my team to think completely out of the box and then again long conversations with them help me. Let me tell you how it works, I insist that these conversations generate that possibility, I go and sit with one of them and we deal with operational issues, certainly on those operational conversations we have the possibility to see ahead, I pushed him to see ahead.

Interviewer: What processes do you have to make your team capable to balance that exploration and exploitation issue? Is it important to you or it's not?

Carl: It is important, not as frequent as I wish, but for example, we have meetings each Monday, and I provoke spaces for reflection and more explorative themes, to analyze "where we are", instead of only concentrate in operational issues.

Other directors, like George, Carl, James and Albert, promotes exploration delegating and assuring that support areas and teams are doing a good work in order to have time for exploration (this is the second most mentioned extended code E11 with 8 mentions done by 6 directors, as it can be seen in Table 20). Lets see what George comments: 
Interviewer: So in some way you free time from your job to do that? How is it? What is your strategy? Or do you free that time outside Tec? Because I understand that most of the time here is operative.

George: The philosophy here is to try not to subsidize other teams or support areas doing their activities. Our support areas are that, support areas. If they don't do their job, you stuck in the operative work. That's their work, to support you. If they remove that work, we can dedicate our time to those things (referring to exploration).

Carl claims that learning from past bosses was important, while Laura challenge their team members either taking them out of the comfort zone or keeping strategic projects always in their agenda. In the next extracts of interviews, Laura talk about the importance of pushing the team out of the comfort zone and Mary claim that limited strategic projects is key for ensuring a good exploration:

Laura: For example, when we deployed the vertical workshops, his attention was there (referring to her boss), and I he questioned me and doubted about me because he told me "Laura, you are thundering your teachers, it is a lot of work" and yes, maybe there was an overload of work, but what I expected was fulfilled. My objective was to get them out of their comfort zone and to tell them "This is what is coming, this experiences, the experience that you can give to the student". (Here interviewed is talking about an explorative Project, the Vertical Workshop).

Mary: ... you must keep few strategic projects, who in the business world as a leader, tell me who brings five or more projects strategic projects, nobody...

Achieving ambidexterity means developing exploration and exploitation in parallel, which is such a difficult thing. Many ideas came into the table, when discussing activities or rituals for improving ambidexterity or for promoting exploration. Finalizing, code E, it is important to mention that according to the comments of two directors, accomplishing explorative ideas is not easy, but was demanded because a fast changing world. Referring to March (1991) the two types of learning and activities are incompatible. For those reasons, all the findings regarding this topic are very important and will add great value to conclusions chapter. 
Now that we know how directors promote ambidexterity, and more specifically exploration in their teams, lets try to identify how they achieve exploration and exploitation from their position. Fundamentally, there are two types of ambidexterity: structural and contextual. As its name implies, code Ambidexterity Structural refers to the former, while Ambidexterity Contextual refers to the second (respectively codes F and G in Table 18, an its respective extended codes F1 to F4 and G1 to G13 in Table 20). "Structural ambidexterity argues that organizations can become ambidextrous by organizationally separating exploitative and explorative activities while at the same time establishing a planned level of integration, while the concept of contextual ambidexterity proposes an organizational solution that enables organizations to become ambidextrous without separating exploitative and explorative tasks and thereby invoking costs of coordination and integration" (Schulze, Heinemann, \& Abedin, BALANCING EXPLOITATION AND EXPLORATION Organizational Antecedents and Performance Effects of Ambidexterity, 2008, pp. 23). The interview did not considered direct questions about what kind of ambidexterity was deployed by each director, nevertheless due to the structure of the interview and the deep with which the subject was treated, several extended codes regarding to each kind of ambidexterity arose (Bossidy, Charam, \& Burck, 2002).

Examining the representative percentage and the number of mentions of each reduced code in Table 18 (12\% with 34 mentions for contextual versus $2 \%$ and 6 mentions for structural), it is clear that contextual ambidexterity was the predominant. This situation is not rare, according to what I could figure out in the directors, they do not have specific teams for exploration and specific teams for exploitation, which means that they achieve ambidexterity in a contextual way. When examining those few emergent extended codes belonging to Structural ambidexterity, we can see that just 4 directors emphasized about the importance of delegating exploitative activities to colleagues or to their own team, in order to have enough time for exploration. This was the case of John: 
John: I think I'm privileged, even Tec now lives much exploitation, forcing us to be operational, including the director of the campus, and that would be my complain. I don't get to do those things, you pay me for another things, I'm privileged because thanks to my team, I could have more time to explore, I couldn't do that without this great team.

But nevertheless interviewees were not clear about how the process of delegation was done or if there was a set of activities already defined and planned for those support teams or processes. What is clear is that when referring to structural ambidexterity, delegation is key; Albert even mentioned that the lack of those delegation skills could jeopardize exploration.

As I commented above, after analyzing twice or three times the interviews regarding to ambidexterity topic, it seems to me that directors deployed much more a contextual than structural ambidexterity. Extended codes G1 to G13 in Table 20 are related to a contextual type of ambidexterity. It can be seen that most of those codes are associated to the way ambidexterity and exploration is promoted or developed (analyzed in the above section). According to those codes, ambidexterity is achieved within the same team or business unit, "without separating exploitative and explorative tasks and thereby invoking costs of coordination and integration" (Schulze, Heinemann , \& Abedin, 2008, p. 3). Analyzing the most mentioned extended codes, it can be seen that have of the directors claim that exploration and exploitation (ambidexterity) happens within the same team and that they should be concerned for developing short-term operation or exploitative activities and at the same time thinking about long-term operation or explorative activities (extended codes F1 to F4 in Table 20). Again analyzing those extended codes, it can be seen that they execute exploitative and explorative activities depending on how the current context is, it means that they apply demanding and dynamic approach depending on either the period of the year (whether the needs in that window of time demands more exploitative or explorative activities) or the demanding generated from the indicators to achieve (whether the indicators and the contexts of their position demands more exploration or more exploitation). As we saw above when analyzing 
how ambidexterity and exploration is promoted, also most of the interviewed directors said that exploration activities are promoted within the same team that performs exploitative activities, either promoting conversations that seeks for innovative ideas, or looking for activities or hobbies that promote creativity or holding conversations and best practices meetings in order to promote exploration:

Interviewer: ... is there anything that you do to help your directors to develop the long term vision within their teams?

John: ... I ask them: I want you to talk to your people; to make them put their head up, so they can respond, "Where do we want to go? Where we are moving? What will happen to my team and me in 5 years? Where do we want to be?

We have seen how directors behave regarding ambidexterity and how they promote it, but during the interviews arose several comments related to specific situations or behaviors that could thread or jeopardize the exploration or even the exploitation. Those comments belong to code Ambidexterity Threats (code H in Table 18 and its extended codes $\mathrm{H} 1$ to $\mathrm{H} 7$ in Table 20). Even this code represents about 8\% (see Table 18) of the total mentions in this theme, it is important and generated heated comments and discussions during the interviews; it seems that directors were concerned and aware of these situations. The most mentioned threat for doing a good exploration was the excessive attachment to regulations and processes (extended code H6), which according to Carl, James, Albert and Peter, could diminish creativity to innovate which is directly related to exploration (March, 1991).

Peter: ... I think in this I alwas exaggerate (when talking about innovative and risky innitiatives), I like so much to do new things, and obviously now, with this new administration, I have to reduce my innovation side.

Interviewer: Why?

Peter: Because there is an excess of regulations, nowadays I protect my self more much more than before, it is not allowed to fail ... 
Continuing with the threads for exploration, four directors thought that being focused just in accomplish KPI (extended code H7), will diminish the capacity to explore. In the next comment, Mary let us now how the day to day operation has lacked the capacity of developing a long term strategy:

Mary: ... How much time could I have devoted to the strategy? I do not think it has been my brightest period because I was busy, I was tired, I was working out with the guys on Facebook up to $11: 30 \mathrm{pm} .$.

Also in the same line of threads for exploration, Albert commented about the importance of delegation skills for gaining time for exploration. James and Laura also agrees that the excessive number of activities and initiatives, and the reduced time (extended codes $\mathrm{H} 3$ and $\mathrm{H} 4$ ), could also represent a risk for achieving those innovative long term proposals that represent exploration, regarding this James said:

James: ... I think that Tecnológico de Monterrey is limiting us in this process of change because we have so much to do and all running in parallel, that leads us to operate all day....

Finally, and regarding to threads for exploitation, just 1 director, Laura, commented that also the excess of innovation (exploration) could affect the day to day operation.

Directly related to the topics of exploration and exploitation, there were a small number of extended codes that were not classified in the topics above discussed, but that could contain important information regarding the way directors operate short and long term activities. Those codes were joined in the code Ambidexterity General (code I in Table ambi1 and its related extended codes I1 to I5 in Table 20). I will just highlight quickly those comments that are new to the analysis and that could have an importance. For example, when associating in a causal relationship, the exploration and the exploitation, it is clear that for at least 4 directors (extended code I5), exploitation "pays exploration" meaning that the day to day operation should be accomplished and is not negotiable, without it, exploration is not feasible. According to the opinion of John, Frank and Saul (extended code I2), exploration abilities could 
be developed with training and experience. And finally, very interesting to notice that 5 directors mentioned in a explicit way the need of being ambidextrous because the fast changing pace of the environment.

By far we already cover all the main topics related to ambidexterity that will help to answer the research question and the objectives of the thesis. Nevertheless, there are two more topics that emerged during the interview, one is related to the ambidexterity level of the directors' bosses, and the second one is related to the level of ambidexterity of the directors' team members. This last topic emerged when asking about the level of ambidexterity that the successor should have. Regarding the comments about the ambidexterity of the interviewees' bosses (code J in Table 18 and its extended codes J1 to J5 in Table 20), It was found that there were no patterns or definite conclusions about how explorative or exploitative the boss is: two directors claimed that their boss is more explorative than exploitative and two more differ, saying that they were more exploitative than explorative. Three other directors, conclude that their bosses were balanced about the proportion of explorative versus exploitative activities. Regarding the ambidexterity behavior of some of the team members, if we analyze the reduced code Ambidexterity Team and its extended codes in Table 20, we can just conclude that most of the directors' teams members are more directed to be exploitative than explorative; as it was mention, this is just and emerged code and a deep analysis will not be done because it is not one of the main topics for this research. It was decided to briefly mention these topics regarding to boss and team ambidexterity, just as support topics that emerged during the interviews and due to the flexibility of qualitative research.

Finally, we will summarize the analysis of the Ambidexterity theme. Despite the fact that exploitation and exploration requires such different skills, actions, structures, etc., and that both compete for resources and decisions, compromising the potential development of each one (Raisch \& Birkinshaw, 2008) and (Gibson \& Birkinshaw, 2004), we could observe that most of the directors are trying to cope with exploration and exploitation within their own teams (contextual ambidexterity). In 
different proportions, they organize their time and activities to meet all the day to day indicators and at the same time they find moments for making reflections about the future of the institution and promote strategic activities that could provide differentiations for their campuses (explorative activities). As it was seen above, in general, interviewees claim that they spend more time doing exploitation than exploration, and they also believe that finding an exploitative director is easier than an explorative one. We also found different ways that the directors use for promoting exploration among their team members, and also for themselves. The most important thing that they highlighted for promoting exploration, is the fact that they spend time talking and persuading their team for doing exploration, despite all the consuming time of exploitative activities. In a nutshell, John synthetizes the behavior interviewees of this University should have, affirming that "this University is not a manufacturing company, campus directors and division directors are not just operative persons, and they must provide competitive advantages in order to differentiate the University from competence". 


\section{RESULTS.}

After the rigorous and conscious process of analysis of each theme, it is the moment to present the results for the general research question and the objectives. This chapter will present those results, and it will be structured in the next form: first, the general objectives will be presented. Responses for each objective will be provided in deep, and after that at the very end, the results and responses for the general research question will be done. This order was chosen because all the results of the objectives will be useful for integrating the results of the general question, and this way provide and easier and clearer perspective for the readers.

During the analysis section, there were provided several extracts of the interviews, and the analysis always makes reference to the codes and extended codes used to explain content analysis. In the case of this chapter, it will not be necessary to repeat the references neither those extracts that were already used in the Analysis Section. Instead, a simpler approach will be used, where the main information that supports the results will be provided avoiding redundancy. Extracts of interviews will be used just where it is considered a priority to strengthen or clarify ideas from the in-depth interviews or when they come from confirmatory interviews, as we shall see next.

After the analysis of the themes, we have information and certain patterns that will help to respond the objectives and the research question. Then, it is here in this section that information from the confirmatory interviewees, Steve, Clark and Ross, will take a role. Those interviewees provide specific information that allows the research to confirm or contrast the information from the 12 deep interviews. This will provide a kind of triangulation (Jick, 1979) that will improve trustworthiness to the research

\subsection{Objectives}

Next, we will provide the results of the research for each of the objectives. 


\subsubsection{To understand how high level directors behave regarding to risk and uncertainty in the execution of strategic decision-making.}

Regarding to risk, high and medium level directors of the 4 campuses selected showed, in general, a positive behavior towards risk and uncertainty in decisionmaking process. For them, it seems to be important that decisions are made in a fast way to address a rapid changing world. They do not take fast decisions in a rapid and careless way, just for making decisions; on the contrary, they claimed to think about the decision and sometimes they check with partners, but at the end they seem to execute those decisions, because it is part of their positions and because of the commitment that they have with the future positioning of their campuses. Moreover, several of them agree in the fact that they prefer to apologize, rather than not taking decisions. They also seem to work best with bosses who allow them to take those rapid decisions, James commented that once he was mad about a boss who took a lot of time comparing small gaining in decisions that should have been made quickly because of the necessity of that campus; they do not like everyone asking for explanations, which is a situation that consumes precious time. Frank argues that decisions should be taken fast in this changing world, because otherwise the consequence could be the "campus to be out of the market"; this situation is consistent with some scholars that claim that the world is fazing a situation of rapid change, and that means that strategies could be obsolete in the short time, then it is obvious that decision must be done (Peters, Re-imagina, 2005) (Yielder \& Codling, 2004), even when those decisions are often difficult because of uncertainty and complexity (Etzioni, 1989; Shafir, Simonson and Tversky, 1993).

According to Eisenhardt (1999) strategic decision-making requires to choose among options that have uncertain results, and where the final decision will depend on the bounded rationality of the decision-maker, it means his experience and limited knowledge (Jones, 1999; March, 1978). Regarding this, all of the directors but one, 
showed also a positive tendency to take risk in decision-making. Nevertheless, the behavior is not just taking risk "in a dumb way". Even they tend to check two or three times the different scenarios, at the end they "assume the risk and are willing to pay the price of making mistakes with the decisions that they take". Most of the directors were located in high and medium risk levels, meaning that they are willing to take decisions, those that will push their divisions or campus to another level an where they are aware about the price to pay when taking a decision. From 12 directors just one seems to be cataloged as a low-level risk director. There were at least 28 situations were decisions were taking with a considerable level of risk. It is very important to emphasize that those directors are also sensitive to the current situation in their campuses, where competence is not static when trying to be in a better position. This is very important, because it is key for being a good strategist to be aware of competition (Porter, 1980).

When accepting velocity and risk as two factors in decision-making, directors also are willing to check and measure the risk before taking decisions. They are conscious that fail is part of the process, and they are ready to take the responsibility. The "price" to pay when risking is most of the times analyzed and use to be confronted with colleagues, just to avoid very negative scenarios or to be involved in a situation with a big grade of responsibility that could jeopardize the future of the campus; this seems to agree with Eisenhardt and Zbaracki (1992) when they claim the importance of a model of rational action and that time devoted to a decision seems to be proportional to the degree of response, the window of time to solve the problem and the cost of getting the information. When failing, the director's attitude should be to learn from the error, and to support and try to make their teams to learn and avoid similar mistakes.

According to Shafir, Simonson and Tversky (1993, p. 13), a reason-based conception for decision-making has some good characteristics because "thinking of choice as guided by reasons provides a natural way to understand the conflict that characterizes the decision making". Nevertheless, in countless occasions, the decision 
maker does not have all the information, neither all the time nor scenarios for taking the perfect decision, or the one that seems to be the best. As already mentioned in the analysis section, in those cases experience, and intuition or gut plays an important role (Hayashi, 2001). Most of the directors affirm that gut effectively play a relevant role in their decision-making process. According to the interviews that gut or intuition could be a mix of a set of experiences, their vision, and the future that they want to create. That decision-making allows them to take opportunities that others do not support, like the opening of a new career or the decision to fail in KPI for the good of the institution (we will explain both cases later). It is important to mention that regarding using gut and intuition, again directors strengthen the importance of spending sometime verifying and analyzing the decision.

Finally, the confirmatory interviewees directly confirmed all the results that were found in most of the directors. It is important to mention that the three interviewees, maybe because of their higher positions, responded in a more vigorous way about risk and gut, I mean that they were completely sure without no doubt. Two of them, Steve and Clark, were very emphatic when affirming that medium and high directors should not be afraid of making decisions. They said, that every day in those positions decisions should be done, and directors must be aware that each decision could represent a possibility to improve their campus or division, or to be fired.

Steve: ... I think it has to be person who is willing to take risks and tough important decisions, and the most important thing, he should know that the position demand that from him ...

Moreover, Steve points out that in those levels decisions must be done, and that this is the only way to proceed:

Steve: .... In a high level position, it is natural that you could make mistakes, and every day you are in the position to be fired due to your decisions, but if you are not aware of that or if you can not handle that then there is a big problem; and there are two possible behaviors: one that you always be stressed or the worst that you can not take decisions... 
In sum, directors claim that it is important to manage risk and uncertainty in these kind of positions. Gut and the past experiences, also are important when taking decisions in scenarios where information is not complete, or when the time is short.

\subsubsection{To understand what are the most relevant directors' factors or characteristics that promote or inhibit ambidexterity, and how it affects the organization}

From the analysis results it is clear that achieving ambidexterity is important for the interviewees. Most of them claimed to manage at some extent a proportion among exploration and exploitation activities in their positions. Also, most of them said that they were more dedicated to exploitative activities than to explorative ones, in an approximately ration of $30 \%$ for exploration and $70 \%$ for exploitation. They were more dedicated to exploitation because most of their KPI and demanding's comes from exploitative indicators, where the day to day operation must be done in order to succeed and continue at the position. Besides proportion of time dedicated to exploration versus exploitation is considerably less, most of the directors claimed that time invested in explorative activities had a higher impact in the performance and the strategy of their campuses, compared to the exploitation activities that satisfied the day to day operation. Despite dedicating more time in exploitation, several of the directors also believe that their skills and preferences were more towards developing mid to long term strategies and activities (related to exploration), than short time ones (related to exploitation), in a similar proportion $70 \% / 30 \%$ but inverted, it means $70 \%$ to exploration and 30\% to exploitation. One of the confirmatory interviewees, Steve, said that despite being $60 \%$ exploitation and $40 \%$ exploration, he was not good "with details" and he likes to see the big picture, the structure of the teams and the way they move towards the future, without being concerned "about the picture of the KPI". 
Steve: I think it should be more on building long-term, long-term design, training and developing future talent, because it will generate better results continuous but especially sustainable results.

In the same line Ross, another confirmatory interviewer, considers himself as an atypical case, because regarding knowing that the day to day operation (exploitation) is necessary for the health of organization, he is always thinking about tomorrow, about those projects that will positively change the education in México and in the world. He would be surprised that leaders in his position could spend $80 \%$ in exploitation because if this happens, then who is going to explore and bring ideas for being competitive in the future? He considers himself as an explorative person, a leader who is in charge of creating the vision and exploring how the future would be; as Raisch and Birkinshaw (2008) point out "a leadership-based solution", an ambidexterity created by leadership.

Ross: ... yes, at night I can not sleep because the financial issued (referring to exploitative activities) but with more intensity I lie awake thinking about innovative ideas to improve México and surprise the world with my students projects (referring to explorative activities).

Another important part of the research was to inquire about how those directors achieved both, exploration and exploitation. Next we will mention two main ways of achieving a balance among exploitation and exploration, and in the next paragraph we will focus in the way directors promote and achieve what they consider the most difficult part, the exploration. According to the interviewees' responses, two main ways to achieve exploration and exploitation were found, the first one highly supported and the second one barely:

i. On one side, most of the directors try to combine and execute exploitative and explorative activities depending on the demand of the indicators (what must be done) and the period of time in which the indicator must meet (when must be done); directors declared that they are constantly switching among those kind of activities, which correspond to a contextual kind of ambidexterity, which proposes 
"an organizational solution that enables organizations to become ambidextrous without separating exploitative and explorative tasks and thereby invoking costs of coordination and integration" (Schulze, Heinemann, \& Abedin, 2008, pp. 2, 3).

ii. In second place, two of them argued that the way to achieve both, exploitation and exploration, was to delegate and trust other areas most of the exploitative activities. Here they were very emphatic that those teams must provide all the support in such way, that they could relay an important part of the exploitation in order to have time for doing exploration. This kind of ambidexterity could be considered more like a type of structural one - which argues that organizations can become ambidextrous by organizationally separating exploitative and explorative activities while at the same time establishing a planned level of integration - (Schulze, Heinemann, \& Abedin, 2008, p. 3) - but without being completely declared, because it seems that the support activities or the different support areas are not planned, neither centrally approved, and each campus does it in their own way.

When discussing about how to achieved ambidexterity and what factors promote or inhibited it, most of them focused only in exploration, taking as granted that exploitation one way or another was going to be accomplished. Regarding to exploration, most of the directors agree that they promote it among their teams investing time in thinking about the future, motivating them to look ahead and innovate and sharing innovative ideas within their team or with other areas in order to gain new ideas and at the same time spreading this kind of practices. This was the most important practice that they mentioned, and in fact, it agrees completely with Clark, one of the confirmatory interviewee, and the person who held the highest position in the organization, who said that:

Clark: ... sometimes we (his boss and his team where Clark was included) spent several hours talking about the future in an informal way, we did not know what was the aim of the meeting, but now I know, and I am repeating this process with my teams ... 
In the same line, the confirmatory interviewer Ross, affirms that his way to do innovation (exploration) is not doing it by himself, but motivating others to do innovation.

The second most mentioned practice that the directors pointed out was the practice of activities that could trigger the imagination in order to come up with explorative initiatives; this is the case of Bob Lutz, President of Chrysler in the 1990s, who had brilliant ideas to help the company in difficult times while driving during a weekend. In third place, directors again strengthen the importance of being capable to delegate those low value exploitative activities to reliable teams, in order to free up time for exploration. Same directors claim for themselves that, regarding the amount of exploitative activities, they always try to invest time in acquiring new information (through activities such as reading, traveling, talking with other persons) and time in "thinking" about the future. Clark resumes it very well when he said that reading, traveling and learning from other persons, was the best way to catch new and innovating ideas that could work in the organization in order to create differentiation.

Summarizing, the main reasons that would promote ambidexterity, and more specific exploration are:

- Investment of time for talking about exploration with team members.

- Investing time in reading, traveling and acquiring new experiences that could be brought as new explorative initiatives.

- Investing time in doing those hobbies or activities that triggers our creativity.

- Investment time and resources to create good performance teams that can address explorative and exploitative activities.

- Delegation of exploitative activities to other teams or areas. 


\subsubsection{Emergent topics}

Two important emergent topics that worth it to be discussed appear during the interview, pushed by the relevance of them and the relevance for the interviewees.

\subsection{Ambidexterity in higher level directors.}

Interesting results arose when the interviewees discussed about the kind of balance between the exploration and exploitation when discussing about higher-level directors, it means those that are above them in the organizational chart. Regarding to this topic, most of the directors think that it is mandatory for those positions to be more explorative than exploitative. They claim that for directors, the higher the position in the company, the more exploration it is required, because directors in those positions are demanded for strategic initiatives that could provide sustained competitive advantage (Ambrosini, Bowman, \& Collier, 2009). In general interviewees consider much more difficult to find explorative directors than exploitative ones, and maybe that's why they consider that exploration is most important in those very high positions. That concerned also emerge from their awareness about the complexity of nowadays environment.

Despite the above bias to exploration in high level positions, it is important to point out that, respondents strengthened the relevance of exploitation as a "must" skill for those persons that could occupy their positions. Besides that, they always reinforced the relevance of exploration for the success in the future.

\subsection{Ambidexterity threats}

Finally, the second emergent topic has to be with the explicit concerned of directors regarding to those risks or threats that could jeopardize that ambidextrous balance, 
but even more that could result in a lack or not sufficient exploration to address the complex future. Just as happens with the theme of risk and uncertainty in decisionmaking, several directors said explicitly, that an excessive focus in accomplish regulations and processes, could certainly diminish the capacity of innovate and explore; also pointed out that when trying to get all KPI directors could forget to "sense" the environment and plan for the future. Concerning this last, John is very clear when he defied his boss regarding to two KPI, telling him that he will not accomplish them because he could jeopardize the future of the campus, he took the risk and stick to his idea, even when he knew that those KPI will not be accomplished and his performance will be affected. Finally, another thread has to be with the lack of enough time for accomplishing all the new initiatives that this on going strategy was demanding from their teams; they were afraid of being out of time for doing exploration.

\subsubsection{To Understand whether Ambidexterity and Risk, Uncertainty and Gut in DM is related with the performance and how it is affected.}

\subsubsection{How risk and uncertainty affects the performance of the organization}

We already commented results about the behavior of directors when doing decisionmaking in strategic decisions. Now let's discuss the results about how risk and uncertainty affect the performance. When we refer to affect the performance, we are referring to those strategic decisions that could affect directly the positioning of the campus and the creation or development of competitive advantages (Eisenhardt \& Zbaracki, 1992), those that require a high level of conceptual understanding (Drucker P. F., 1967). For addressing this part of the objective, it was essential to go and back several times, in that spiral process that Creswell (2003, p. 183) claim as part of the nature of qualitative analysis, through the content of the interviews, looking for those sections where risk, uncertainty and gut played an important role in directors decisions or actions. 
Risk and gut affects performance according to the interviewees. In the case of one of the campus, John skipped a direct order to accomplish at least $85 \%$ of graduated students with an abroad experience because that decision would affect the campus' budget, and hence the number and quality of professors, which seems to be his priority. Gut leaded Mary to decide the opening of Marketing and Communication career, despite the negative advices of merchandising companies, which tried to convince her about the contrary, no she is having success with this career. In the case of Albert, he decided to take the risk of firing more than $20 \%$ of his directors in order to have a new team that could address the coming challenges.

Albert: ... yes, yes I have done that kind of decisions, this simply because right now there are $20 \%$ fewer heads compared to the time when I arrived to the position.

Interviewer: Were you forced to do that or was it your decision?

Albert: I think both, but also the pressure to improve my division.

Not all the decision that involved risk and gut positively thrived; Peter's strategic decision to offer all courses of International Business Bachelor in English language offered a challenge that could not be accomplished, nevertheless it resulted in an increase of full time international professors, and hence in an improvement in academic quality, an important competitive advantage. Next it is show how this risky decision has its bases in the need of the development of a competitive advantage (Porter, 1980). In this decision, we can also see the impact of fast decision-making:

Peter: Well, the decision to declare that all the courses will be taught in English for the International Business Bachelor degree, that decision did not work at $100 \%$.

Interviewer: Did you declare 100\% of courses to be taught in English?

Peter: Yes I did declare such thing, without knowing the size of the decision and the future problems.

Interviewer: Did not you analyze the consequences of that decision?

Peter: I had to take it, otherwise we could be out of the market, we must move forward, people want to see and perceived a differentiated product, at the end we had better professors. 
Risk and uncertainty, but most of all, the rapid changing world, pushed Saul to put in risk some KPI in favor of hiring new international professors for improving academic quality. Steve, one of the confirmatory interviewees, shows how fear to assume risk in strategic DM could affect the performance of an organization when pointing out that: "the pressure of a good picture in the KPI and the fear to fail, could lead a director to act making just minor incremental adjustments to improve, leaving aside those big necessary decisions that could create the difference for considerably improving the performance of the organization" (mentioned by Steve). All the mentioned examples and several more (there are about 22 examples) constitutes an example of the performance being affected by risk, gut and uncertainty in strategic decision making. On the next section, we will bring to the table more examples where risk and ambidexterity shows a relevant role for the performance.

\subsubsection{How ambidexterity affects the performance (or the strategies)}

According to Schulze, Heinemann and Abedin (2008) it seems reasonable that ambidextrous organization are more likely to achieve competitive advantage than those that are just worried to accomplish either exploration or exploitation. Then the first question that arises is whether the interviewees were concerned and occupied in trying to achieve ambidexterity, and after doing the analysis section the response is yes. They try to balance day to day operation with windows of time for doing exploration; either trying to delegate activities to different teams - structural ambidexterity - or, the most common, trying to be efficient switching between two kinds of activities -contextual ambidexterity-. Then lets resume how behavior regarding to ambidexterity affects performance. As we said most of the directors claimed about a contextual ambidexterity where they as leaders develop somehow supportive context for achieving (Gibson \& Birkinshaw, 2004). In that search of balancing of activities they are convinced that exploitation pays exploration and try to push their teams to keep a healthy operation and at the same time bet for developing new initiatives. 
We shall see several examples that effectively confirm that being ambidextrous could affect the performance, and in this case it seems that in a positive way. Several studies agree that being ambidextrous is positively related to a better performance in the organization (Schulze, Heinemann, and Abedin, 2004; Raisch and Birkinshaw, 2008). It seems that for the analyzed campuses, high level directors such campus directors and division directors, are in charge of the proposal and execution of new ideas and hence the exploration, in concordance with a top-down approach where knowledge inflowing from persons at higher hierarchical levels are positively related to exploitation (Raisch \& Birkinshaw, 2008). During the examples, it is important to notice that some of those projects are planned to initiate in the near future and others are running. Execution is also important regarding to exploration (Bossidy, Charam, \& Burck, 2002), not jus having the idea and shaping the future but building it. In this sense, all of the directors but Mike claim to be working on the differentiation of their campus, thinking in what area (such as automotive, aerospace, etc.) should they be focused to differentiate; they also claimed that they had such a limited amount of time to dedicate because of the day to day operation, but at the end they were concerned about doing exploration and balancing to two kind of activities. Next we will provide selected examples that have the aim to show how being ambidextrous impact the present and future performance of the organizations. It is important to mention that, risk and gut also played an important role moreover in those innovative initiatives.

John, for example, said that thanks to the fact that he developed a high committed and performance team, he has the opportunity to explore, to talk with their team and to sense the environment and the competence, and propose strategic initiatives. On the contrary, Saul said that during the first year as a new division director, more than $90 \%$ of his time was spent in doing exploitation, putting aside all the strategic initiatives that he had in mind. That desire and consciousness for exploring new ideas and pushing hard to accomplish them could also make a difference for the campuses. Again John pushes hard for a big idea that could create a differentiation in the future for his campus, an innovative and difficult to realize idea - typical from 
exploration stage -, and as it will be shown, that was a non planned initiative and even with no budget assigned, but it is clear that he has a vision, assumes the risk and that he exploit and explore:

John: ... no, then if there is a great project and I do not want to wait for executing neither stop it, then Where are we going to get money J.J.? (J.J. is the person in charge of the money) It is your problem be creative and got the money please ... then he already has that dynamic. And he knows that those freak ideas are part of me, and the day to day operation is not compromised it is also accomplished, but I will not stop my sand of drones project and right now and it costs about 100,000 dollars, then let's go, let's do it.

James resumes very well the meaning of ambidexterity and how it would shape and affect the present and the future of the organization:

James: ... and as leaders we must learn to selectively forget the past, remove those practices that are not all good, manage the present but bring the future. That's what we are, selectively forget the past, you manage the present and meet with indicators, but the management of this are building the future.

Besides operation George is also thinking about the future, those strategic projects that could differentiate, he is willing to take risk and to explore, and as it can be seen in the next project, it affects the performance of the organization:

George: I am planning a draft of a great project for the new career in business, I've been working for one year and my idea is that by summer 2017 make the first pilot under the scheme of iSemester, which is not authorized yet, nor I have proposed, but since it is complete I will propose it and hopefully it will be accepted. The proposal will come up with evidence that, if it works; those are the things that I'm seeing in the long term ...

Laura for example, tries to cope with new innovative ways of teaching outside the classroom implementing Vertical Workshop, despite all the operation that she has to do. She is convinced about this kind of activities in their strategy, and she affirms that those big projects create a better performance environment among their professors, 
and at the same time students like them so much that they represent a competitive advantage. She was willing to take the risk of leaving aside other indicators or even having annoying professors because the amount of work. At the end, she had some of them mad, but the balance was positive. It is worth presenting exactly what she said, because you can read the passion and the way she trusts and speaks to this exploration for the future:

Laura: Look, I tell you this example of vertical workshops, which is my closest example. I performed Vertical workshops for professors to live the experienced, to take them out the comfort zone and to introduce them in new educative model Tec21, which has cost me a lot of work to implement in business school, but this decision had consequences. There are things that I did not saw coming, for example, that professors would get sick because the degree of stress of them would be so high that they were going to get sick, I did not see that coming. On one hand my intuition said it is good to implement, but on the other side ignored consequences, I mean, I did not take it into account. Another thing I did not see but that is not something that happened, the teamwork. There were things that I discussed and analyzed, for example I will prepare professors for model Tec21, I will generate great experience for the students, I'll gather everyone in one project, but I did not consider the stress caused to people.

Peter on his point of view, claims that exploration permits the campus to differentiate with initiatives that are complicated to be copied:

Peter: ... I think the iWeek and iSemester (new educative model activities) both are such innovative activities that nobody is going to be able to implement (referring to competence); and when they will be able to copy, because they could make it at some point in the future, we would be implementing with initiatives even harder to copy ...

But not all the projects bring positive results explicitly. For example, Peter decided to bet for the future trying to differentiate his division through the hiring of 6 international professors. When those professors were operating, he realized that they do not agree with the vision and the way of working of the institute, and the project failed with the resignation from 5 of those professors. It was a risky initiative, and explorative approach that did not work. But when we went deeply in the 
interview, Peter realized that this failure and the experienced gained set the bases for being the campus with the most international faculty (the one with most international professors).

We could refer more examples like the ones above; in fact there are about 23 identified examples. On those examples, it is clear that performance, moreover in the long time, would be affected if the directors lacked the sense of balancing exploitative and explorative activities; performance also would be affected if the directors are afraid to assume risk and the consequences of failures. Let's see what Steve (a confirmatory interviewee) said about that:

Steve: You have to take decisions for the benefit of the organization, because trust is placed in you, and people will recognize that in the future when they say "hey, this guy fixed the broken things, he took decisions, he corrected the situation".

Most of the explained projects, regarding they succeed or not, provided a new set of characteristics and strengths to the division or to the campus. If directors would be averse to risk, or if they would not have be concerned about achieving a balance on exploitation versus exploration, then those initiatives would never borne and the performance of their division or campuses would be affected

\subsubsection{To understand how directors perceived their own strategy and how is it related to University strategy.}

Regarding to strategy, there were such different opinions in the way division directors and campus directors perceived strategy. As many as to generate more than 80 extended codes related to all relevant comments regarding to strategy and differentiators. Next, I will summarize the findings that resulted from the analysis section. Again, it is important to strengthen that texts of interviews were read several times in order to extract the essence of what the respondents were saying about strategy. Before starting, it is important to mention that we will not make reference to extracts of interviews of codes because, that analysis was deeply done Chapter 4 . 
Regarding to the general University strategy, and when asking how do they perceived the university strategy, most of the directors responded that strategy was concentrated in 2 main areas:

- First, the strategy is conducting a radical change from autonomous campuses to "one TEC" meaning one university that is governed by common processes.

- Second, the strategy is based in improving the position and the international rankings of Tec de Monterrey through the implementation of a quite new and disruptive educative model, called TEC21, which is based in the attraction and selection of the best professors and the best students.

Ross, one of the confirmatory interviewees, synthesized very well the strategy in the next extract, but also showed his concerns about the complexity of the unification of the campuses and the change of paradigm to processes and the lack of communication from strategists to leaders like directors of campus and directors of divisions:

Ross: I will separate in two areas: first is audacious bet, that will bring as a consequence of having the best professors, students and careers (it means a new educative model), that the university scale position in international rakings, I am happy with this part of the strategy. On the other side, the search centralization through common processes and operating regulation among campuses, having an institution oriented to process, the use of information systems for management. Regarding to this part I am not completely convinced, because this type of transformations are not easy to achieve, a few firms had succeed, but as part of this organization I will support this process.

When asking about the strategy of the University, Steve (another confirmatory interviewed) responded in a general way, without mentioning specifically the aim of the strategy; but what he made very clear is that this changing strategy was very 
necessary in order to increase the chances of the university to survive and being positioned among the best ones:

Steve: ... today much of what we do will put Tec de Monterrey as over time always reconfiguring to be in a better competitive position also necessary today worldwide, and that helps us be a good college or best in Mexico if finally today and in all industries are exposed to the entire global environment.

Ross was not the only one to be skeptical in relation to this centralization, several directors expressed their concerned as we shall see in the emergent topics in this section. This could be normal; moving a big organization to a disruptive change is not easy, even less when the organization is in a successful position (Kotter, 1990). Nevertheless, it is important to mention that most of the directors had a positive attitude to the strategy at the long term, and also that they knew that this change was necessary due to the complexity in the environment. Also they pointed out the relevance of creating our own strategy, avoiding the trap of copying others that could not work due to the particularity of each university, especially in Tecnológico de Monterrey. Regarding the term of accomplishment of the strategy, most of them consider that this strategy is medium to long term, thinking in a period of 5 to 10 years more, as confirmatory interviewee Ross affirms.

Concerning to the strategy of the directors, whether they are leading a division or a campus, they perceived their strategy mainly focused in three major areas:

- First, creating differentiation having an excellent academic quality through the attraction of the bets professors and the training of the current ones: they focused in international professors from different countries and professors with wide practical experience in different industries. This could be more part of an induced strategy, where according to confirmatory interviewees Clark and Steve, both initiatives are part of the University strategy (Burgelman, 1991). 
- Second, creating differentiation through the creation of innovative experiences and educative processes, based in the development of ecosystems of careers and educative model TEC 21 (iWeek, iSemester, Vertical Workshops, etc.). In this point, the strategy is a combination among an induced and an autonomous strategy; induced because elements such iWeek and iSemester are mandatory from the top management; autonomous because initiatives such as increasing in internationalization of students and Vertical Workshops are created inside the campus and are initiatives coming from the leaders in this case the directors (Burgelman, 1991). Also, an important part of this strategy is the quality of service and the satisfaction of students. They must feel challenged and at the same time well treated, according to Steve.

- Third, the creation of value and differentiation through the seizing of regional strengths through the "vocacionamiento" of the campus, that means the process of defining and concentrating in specific areas that could be unique and difficult to imitate (Wernerfelt, 1995); some campuses are thinking about automotive, other in agro industrial sector, others in entrepreneurship or manufacturing, among others. In this strategy, it could be noticed that different directors are concerned about finding the right area of "vocacionamiento"; nevertheless, there are directors like John who is very advanced in this strategy and others like Saul who is just starting. This strategy is more autonomous and also tries to find niches that could address "blue oceans" (Mauborgne \& Kim, 2005).

\subsubsection{Emergent topic: Threats and Risks for the University Strategy.}

When analyzing the interviews, I clearly noticed that almost all of the directors expressed in a reiterative way, several threats and risky situations that could jeopardize the accomplishment of the current strategy. But those comments were not only to point out threating situations, but also to complain about certain situations that represent obstacles for the deployment of their own strategies. 
All of the interviewees but Joe made comments regarding threats or risks for the current strategy. After the analysis of the extended and reduced codes in Chapter 4, we categorized six main threats and risks that the interviewees pointed out:

- The lack of a correct communication of the strategy from top-level directors.

- An Incorrect strategic planning, which causes several activities to be running in parallel, compromising the quality of the strategic deployment, the capacity of exploration and exploitation and even can cause people to burnout.

- The excessive centralization of processes and the creation of complex routines, which causes that people loose the initiative to innovate.

- The mismatch between the profiles of new generation of students and the new educative model.

- The cost of the new educative model, which seems to demand more full time professors, making impossible for some campus to pay the cost.

- An excess of innovative activities that could cause to diminish academic quality in the search of satisfying the "market".

Those concerns are not just coming from the 12 interviewees. Also confirmatory interviewees, who hold higher positions, are also concerned by some of the above mentioned threats and risks. For example, Ross is clearly pointing out the threat of a poor process of communication and the fact that campus directors are not being taking into account when making decisions that affects directly to the campus. $\mathrm{He}$ claims that big changes require leaders that are willing to be near people, communicating and supporting.

Ross: ... While the leaders who make the decisions do not consult those who operate and while not assume their role of communicate processes and change management, there is a 
very high risk that things do not happen and when as leader you are not aware of the complexity of these decisions, there are frictions and conflicts, and that's not good.

Summarizing, it seems that directors are aware of the current rapid changing environment that could jeopardize any strategy in almost any industry, including education (Wind and Main, 1998; Casares et. al., 2015). Directors seem to deploy a mix of induced and autonomous strategies that are aimed to create sustained competitive advantages in order to differentiate from competition. In the four campuses, it seems that those strategies are aligned to the general strategy of the University, but in different ways of execution. Despite the negative opinion regarding the process of centralization, it seems that directors are still operating autonomous strategies and their own differentiation always trying to be aligned to the policies and regulations that are now common to all campuses.

\subsubsection{To understand whether ambidexterity and/or risk and uncertainty in strategic DM can influence dynamic capabilities.}

The response to this objective is probably the most challenging one, because of the difficulty in defining specific questions in order to inquire about the deployment of the inhibition of them; we can not ask the audience whether they created dynamic capabilities, because they are dynamic and in occasions they are created without planning, without predicting the outcome (Eisenhardt and Martin, 2000; Eisenhardt and Zbaracki, 1992; Burgelman, 1991). I did not explain the concept of DC to the interviewees, neither did I mentioned any question with the concept. The aim was to find results, if any, from the questions regarding to the main three themes: strategy, risk in DM and ambidexterity.

Dynamic capabilities and Resourced-Based View of the firm gain importance until the 80s triggered mainly by three reasons: the growth of a more unstable environment in a rapid changing world: second, the flourishing of the idea of competitive advantages rather than industry attractiveness as the main source of 
profitability; and last, the existence of a world where costumer preferences and new technology are volatile (Grant, 2010). Under these circumstances, firms began to realize that they should count with valuable resources to compete, VRIN resources according to the theory of RBV (Barney, 1991) and to dynamically exploit and change the reconfiguration of those resources in order to generate sustained competitive advantages in the mentioned dynamic world (Teece, Pisano, \& Shuen, 1997).

There are several factors that contribute to the deployment or the inhibition of dynamic capabilities, such as the level of external dynamism, the pace of the specific industry, the type of leadership, among others. Nevertheless, managers (in this case directors) play maybe the most important role, similar to that of a card player where the player has cards determined exogenously depending on the environment (external factors). Success in the game depends on how the player takes advantage of those cards, and the subsequently acquired cards (endogenous factor) (Lockett, Thompson, \& Morgenstern, 2009).

Then, to understand whether risk and uncertainty in decision-making or ambidexterity behavior are related to the deployment of dynamic capabilities the first question that we should arose is whether directors (referring to the interviewees) are aware of changing environment and if they consider that they have VRIN resources in the Institution. Going back to Chapter 4 and also to above results of the first 4 objectives in this Chapter, we can conclude in general, that both responses are affirmative. Most of the directors agree that HEI are part of the rapid changing world and that their teams represent valuables and unique resources for the organization (Wernerfelt, 1995) (see code D Ambidexterity Team and its extended codes in Table 20).

Then, how can risk and ambidexterity behavior in directors influence dynamic capabilities? According to Teece, Pisano and Shuen (1997) the leadership of top management level is key in the development of DC because they must make decisions, some of them with a considerable level of risk, and those decisions will 
create routines that in turn will deploy and create a changed set of resources - not necessary new resources, but a renovation of the current ones - that will produce new forms of competitive advantage to address the rapid changing world (Ambrosini, Bowman and Collier, 2009; Barney, 1991; Teece, Pisano and Shuen, 1997). The creation of the routines that will create DC require persons who are willing to take risks in their decisions continuously, and willing to trust their gut and path-dependence - it means the previous experience - to explore new alternatives, new ways of doing things, that could never anticipate a $100 \%$ success. That was the case of Tecnológico de Monterrey's President, Salvador Alva, who proposed a drastic change in the strategy of the University even when it was running in a stable situation and well ranked ${ }^{10}$.

According to O'Reilly and Tushman (2007, p. 12), "dynamic capabilities are at the heart of the ability of a business to be ambidextrous - to compete simultaneously in both mature and emerging markets - to explore and exploit". The interviewed directors, including the confirmatory ones, talked about several cases and practical situation where they put in action the essence of development of dynamic capabilities through the deployment of ambidextrous abilities, most of those cases were already addressed and explained in this chapter and in Section 4.3 in Chapter 4, but next we will numerate the most representatives that show the relationship among the assumption of risky decision-making and the managing of ambidexterity in deploying strategies that could be positive for the differentiation of the campus, and that contributes to the creation of dynamic capabilities.

For example when 7 of the directors were concerned in finding the niche of differentiation for their campuses (aeronautics, agriculture, automotive, etc.) - when they talked about their "vocacionamiento" -; even when this activity does not belong to their annual evaluation, they agreed about the importance of seizing the strength of their region to differentiate (Porter M. E., 2008) and, at the same time, to align the

10 From the 2015 Annual Presentation of Salvador Alva, President of Tecnológico de Monterrey, happened in July, 2015. 
teams to a new goal - reconfiguration of base resource, part of the aim of dynamic capabilities -. A second example, when most of the directors regularly invest time to thinking about the future and the differentiation of their areas. A third example, when Karl 20 years ago decided to push for the creation of the first technology Park in the region focused in aeronautics, setting the path for a new way of thinking and operating of his faculty and that according to him, created a set of practical projects that produced a differentiation in his engineering division. A fourth example when Frank is currently searching for budget and support for investing in two laboratories for alternative energy, betting that this area will address the future needs of external enterprises, or when George shows himself enthusiastic by proposing innovative summer programs - where students have to visit 5 different companies in, at least, three weeks for applying the knowledge of two different courses - that at the end were rejected by his colleagues because of the complexity of the project - exploration like this requires a different an inconsistent organizational alignment ( $O$ 'Reilly \& Tushman, 2007) -. Nevertheless, and referring to the last George example, according to him, even when his project was rejected, the proposal about the innovative summer programs improved the "drive" of the professors in such way that now they are thinking and "dreaming" about the creation of bigger educative projects. In the next last example, Peter describe how he fails when trying to differentiate one of the bachelor degrees offering 100\% courses in English; he accepted that he was too risky in his decision, but at the end he declared that even though he failed, the process of trying this risky project came out with an improved faculty with more abroad professors in the campus.

Interviewer: Yes please, if you have mention a decision in which the risk has taken a very important role, which has been a risky, or very creative or very innovative decision.

Peter: Well the decision to offer $100 \%$ of the courses in English (when referring to one of the careers).

Interviewer: Then $100 \%$ courses in English.

Peter: Yes, 100\% courses in English, I declare it without knowing the magnitude of the problem that was to come later.

Interviewer: Did you analyzed or was one of those decisions you said, we have to take it? 
Peter: We must take it, otherwise we could be out of the market, you have to go forward and see how you do, it's like logistics reverse or reverse engineering is already good product, as you did, well, here the same has to be the product so people really see a difference in the market and as you do because that will be the heart of the matter. At the end, we did not achieve the $100 \%$, but in the meantime we ended with better professors.

The last above example shows clearly what the nature of the dynamic capabilities is, sensing and seizing opportunities (the opportunity to offer $100 \%$ of courses in English) for establishing risky projects (risky means that projects are complex and uncertain about the chances to succeed) and make a reconfiguration of the resources (in this case the faculty) in order to create an sustained competitive advantage (that in this case did not happened, but even though a better faculty arose).

Finally, it is important to summarize that all the mentioned examples allow the research to conclude that the behavior of interviewees related to risk in decisionmaking and ambidexterity, provide the basis in the process of the creation of routines for the deployment of dynamic capabilities. Mainly because, the exposed situations contributed not only to the creation of competitive advantages, but also to the reconfiguration of the teams (resources) in such way that they can be "trained" to address an unknown future, a clear process of development of dynamic capabilities (O'Reilly \& Tushman, 2007). The leader, or the director in this case, will largely influence the development of dynamic capabilities because he is in charge of the decisions about those projects that will trigger the possible reconfiguration of the base resources; that is the case of Carl who this year decided to open the Mechanical Engineer career, even against the opinion of marketing experts, and whose decision will bring a change in the based resources of his campus (new professors, new facilities, to leave other projects) and maybe a competitive advantage in the case of success. Just to end, I want to strengthen out that, a large part of the findings are attributed to the flexibility and nature of qualitative analysis and specifically the semi-structured interviews, which as we saw in Chapter3, provide the way to go deeply in the selective search of information (Creswell J. W., 2003); other scholars such Gibson and Birkinshaw (2004) have also taken advantage of this methodology. 


\subsection{Research Question}

Next, we will provide the results for the research question.

\subsubsection{How high level directors behave regarding ambidexterity and risk in strategic decision-making and how they are related to performance in 4 campuses of a high cost multi-campus private university in Mexico?}

Very interesting and rich results were found about the research question. Because the objectives were defined to respond the research question, and due to the similarity between them and the research question, most of the results in this section will come directly from the results of the five objectives that were responded above. In order to avoid duplicity in the analysis and the results, while answering the research question, in some occasions above analysis and results sections will be referred and just a few necessary and complementary illustrative examples joined with a synthesis will address the research question.

It has been found that ambidextrous behavior is related with performance in a firm, and to its chances to survive in this rapid changing world, as March (1991), referred in Schulze, Heinemann and Abedin (2008, p. 1), pointed out "in order to ensure longterm survival, organizations are forced to constantly generate new competitive advantages, which underscores the importance of balancing the exploitation of old certainties and the exploration of new possibilities".

We found that almost all of the directors, but one, simultaneously developed exploitative and explorative activities, showing an ambidextrous behavior. Several examples and practical cases were provided Chapter 4 and the above sections of this chapter. Regarding to exploitation, interviewees claimed their concern about the importance of high and medium level directors knew how to implement and deploy strategies and tactics in order to achieve the day to day operation, the 
accomplishment of KPI and the financial health of the institution. As some of they said, "exploitation pays exploration", and it is not negotiable being well evaluated in the short term (referring to exploitation); as Levinthal and March (1993) in Raisch and Birkinshaw (2008, p. 377) said, long-term survival depends on the ability to "engage in enough exploitation to ensure the organization's current viability and to engage in enough exploration to ensure future viability". Directors claimed that, in general, they spend more time exploiting than exploring; some of them manage a $80 \%-20 \%$ proportion and most a $70 \%-30 \%$ or $60 \%-40 \%$ proportions. As John said, it demands a lot of time to implement good ideas:

John: I'd believe that a balance in that model you mentioned, ambidextrous, I'd imagine $70 \%$ in operation and $30 \%$ in exploration would be very healthy. I mean, if I'm dedicating a third of my life in exploration I need two thirds of my life to put it in practice, evaluate it and have feedback. A 50\%-50\% I would not imagine it because you'll generate a lot of information that you won't be able to put in in practice.

Although the directors said that they devote more time to exploitative activities than explorative ones, in general, they perceived themselves more as long term oriented persons (explorative) than short term (exploitative); and not just because of their profile, but also because they seems to like and enjoyed so much exploration over exploitation. As John, Carl and Peter said: "I wish I could spend more time doing exploration". Interviewees also agree that high level positions demand more exploration than exploitation, and more exploration skilled persons than exploitation ones. In the same line, they also agree that it is more difficult to find good explorative directors than good exploitative ones. Confirmatory interviewees, also agree about the importance of directors were good in that balance among exploration and exploitation.

Concerning to how those directors promote the ambidextrous behavior and more on the exploration in their teams, in general they agree that they perform both, exploration and exploitation, within the same team, switching activities depending on three factors: the demand of the different type of indicators to achieve, the context 
of the campus or the division and the time of the year. This way of achieving ambidexterity corresponds to a contextual ambidexterity, where "the context is dynamic and flexible enough to allow individuals to use their own judgment as to how they divide their time between alignment-oriented and adaptation-oriented activities, and both are valued and rewarded" (Gibson \& Birkinshaw, 2004, p. 211). Nevertheless, that way of dividing activities between exploration and exploitation depends largely on the guidelines of the leader, and for those reasons, interviewees said that the most difficult part to achieve was to make their teams and themselves explore. Regarding the way of promoting exploration, they and also the confirmatory interviewees agree in mainly three different ways: spending time talking with their teams about the future, and making them think an reflect about the future position of the campus and the competitive advantages that they should create; practicing activities that could trigger the imagination in order to come up with explorative initiatives, sometimes those are hobbies or activities that keep them challenge; and third, delegating effectively in order to liberate time for doing exploration.

According to Gibson and Birkinshaw (2004) "senior executives likely play a role in fostering ambidexterity, primarily by encouraging and nurturing adaptability". In this context, directors should be making strategic and important decisions constantly and in a good pace, in order to balance the tension among explorative and exploitative activities. Due to the fact that in order to explore, they also should be making decisions surrounding by uncertainty and risk, and then it is important to figure out how directors behave regarding risk in DM. Regarding risk in decisionmaking, most of the directors exhibited a medium to high level risk management when taking decisions that could affect the performance of the campus or division; it means that they are able to manage the uncertainty of some decisions, and that they are aware that decisions should be taken regardless the lack of information or the certainty of success. They also agree that decision should be taken "fast", meaning that they should not be leaving "in the table" for long periods; they strengthen the idea of checking possible sceneries and even discussing with colleagues, but at the end they know it is their responsibility to execute those decision, mainly because of 
the nature and level of their positions and because the fast changing environment is pushing them to seize the opportunities and to take risk. Directors seem to be aware of that, as Frank shows in the next brief extract of his interview when referring a risky project in his campus: "... we must take it, otherwise we could be out of the market, you have to go forward and see how you do ...". As we pointed out, they seem to manage the risk and execute fast decisions, but checking and considering the consequences of those decisions. When they took that kind of decisions, directors argued that gut and intuition always played a very important role. They conceived gut as a set of experiences and knowledge that has being created through experiences. When trusting in their gut, some directors as John, are even willing to fail in KPI, just to achieve those projects that they "feel" could represent in the future a competitive advantage:

John: ... of course I will not reach that number (when talking about the percentage of students that should have an study abroad experience); if I do that I will loose some money, and I need that money for hiring more and better professors. Academic quality is the first thing at campus, there is no way I will accomplish that KPI ...

Are ambidexterity and decision-making behaviors in high level directors related to the performance in organizations? Several resources indicate that they are. Top management and the way they lead their teams and make decisions, could develop or inhibit the balance among exploitation and exploration, and even the deployment of dynamic capabilities; according to O'Reilley and Tushman (2007) when refer to (Eisenhardt and Martin, 2000; Lavie, 2006; Teece, et al., 1997;) "dynamic capabilities emphasizes the key role of strategic leadership in appropriately adapting, integrating and reconfiguring organizational skills and resources to match changing environments". Then it is worth to ask how ambidexterity and risk in DM behaviors are related to performance in 4 campuses of a high cost multi-campus private university in Mexico? Most of the responses to this question were largely addressed in the above Chapter 4. Back to that section we can see that most of the given examples (projects related to innovation in educative models, the definition of certain areas for the future, the creation of Technology Park, the bet for a new 
faculty, the opening of new careers, etc.) requires from the directors a precise and decided decision, several times even against chances and recommendations from externals. Decisions that could shape the future strategy of their division or campuses, but decisions that they took trying to look for competitive advantages that could set them apart from competition. In order to avoid repeated examples that were already mentioned and discussed, we will use two illustrative examples that show in an implicit and explicit way, how ambidexterity, risk in decision making and performance (strategy) are related.

First, I will refer Laura when she commented about the implementation of new educative experiences, the Vertical Workshop, which demanded the participation and coordination from all of the professors and students in her academic division in order to execute a 6 months project. This project was not linked to the regular courses, and represented a commitment and effort additional to the day to day operation of every one. Laura expressed the inconformity of his boss about the deployment of this project:

Laura: For example, when we deployed the vertical workshops, his attention was there (referring to her boss), and I he questioned me and doubted about me because he told me "Laura, you are thundering your teachers, it is a lot of work" and yes, maybe there was an overload of work, but what I expected was fulfilled. My objective was to get them out of their comfort zone and to tell them "This is what is coming, this experiences, the experience that you can give to the student. I want to prepare students and professors for the new educative model TEC21 and I also want my students to improve their skills in order to be more attractive for employers". I want the professors to challenge themselves and to create new experiences for students...

Here, Laura decided to take a risky decision about the implementation of complex project in which she trusted blindly (I am making these asseverations because I remember during the interview the attitude and passion in her voice and her corporal manners). In her mind, the project would represent an opportunity for the creation of a new and attractive educative experience for the students. According to her boss, she failed in the project, because it "just created dissatisfaction among their 
professors, and even some of them got sick because the stress". But Laura was not seeing just the project, she was looking "beyond her nose", she was projecting a future, a future where the professors started to propose new and attractive experiences for the students (she wanted to change the mindset of professors, a change in resources for increasing the number of explorative proposals), which in turn could constitute a competitive advantage in her division, which in turn could create a differentiation among competitors. Without even being aware of that, Laura was in the track of deployment of dynamic capabilities, proposing routines that could change the resources in order to bring competitive advantages (Ambrosini, Bowman and Collier, 2009; Eisenhardt and Martin, 2000).

The second example corresponds to John, who has proposed for this year the creation of a drone laboratory for experimenting possible projects in the future. John decided to drop out the accomplishment of an important KPI (internationalization of their students) in order to have money for the drone laboratory:

John: ... no, when there is a bi project, then I always say Jesse, How are we going to get the money? In this case, I already toll by boss, no I will not achieve that KPI, no, no, no we are not going to achieve that KPI, because if affects by budget, and I need that drone laboratory, and that is the way I try to realize that kind of projects. And then, there is, I have the drone lab., and What if we need a new machinery for automation, or a laser cutter, then we must get the money, because that is the important, what about my office? Please it does not need to be remodeled, that can wait ...

As it can be seen, it is clear for John that academic quality and state of the art equipment and laboratories are the priority. He is willing to put aside KPI that are relevant to top management, but not as important as those projects that, according to his vision and intuition, could represent a competitive advantage in his campus. He is aware of the complex challenges that Higher Education Institutions are facing nowadays (Yielder and Codling, 2004; Timberlake, 2004; Tavernier, 2004), and that one of the ways to survive is to continuously create competitive advantages that could differentiate from competence (Ambrosini and Bowman, 2009; Porter, 1996). 
Summarizing, we could see a lot of examples such as the two above, where a mix of deliberate or induced and emergent or autonomous strategies (Mintzberg \& Waters, 1985) arose from different division and campus directors. As we already mentioned, HEI are facing complex and risky sceneries (Lorange, 2002; March and Cohen, 1974). Regarding to multi-campus universities, such as Tecnológico de Monterrey, "managers must maximize the quality of both operational and strategic decisionmaking. Managing operational decision-making requires attention to timely service and timely decisions, while maximizing strategic decision-making requires planning processes, which create buy-in" (Timberlake, 2004, p. 97). In the case of the interviewees, they are aware of that and they are pushing hard different initiatives in order to create competitive advantages and at the same time to reconfigure their teams to address the future. They are working for balance exploitative and explorative activities. Also, they are willing to face and deal with risk and uncertainty, particularly when doing exploration due to its experimental uncertain nature. But, at the end the way they behave regarding ambidexterity and risk in decision-making affects the performance 


\section{CONCLUSIONS, IMPLICATIONS AND FUTURE RESEARCH.}

This research represents the opportunity to bring together two themes that have been broadly treated and that could affect the strategy, not just the deployment but also the execution, and therefore the performance of organizations. In this late section, we will discuss the conclusions and implications of the analysis and results of the research question and the objectives, and ideas for future research work.

One aim of the research was to explore how directors behave regarding to risk and uncertainty in DM. There is no doubt that big decisions are sorrunded by risk and uncertainty (Shafir, Simonson, \& Tversky, 1993) Regarding this, directors make important decisions in an agile way, knowing that decisions must be done in order to have results, and also they, in general, are persons that know how to cope with risk and uncertainty. This is important in a positive way for strategy and performance of organizations, moreover due to the fast changing environment in which we are now living, just like commented by Wind and Main (1998), referred in Balaton (2007), when affirming that the passivity (referring to non agile decisions and the procrastinating behavior) is the most risky strategy nowadays. Directors are not afraid to take decisions that could jeopardize momentarily indicators or the status quo of the organization, they are willing to take the responsibility on their decisions and when they have to trust their gut, they do, in the search of those risky decisions that could benefit the performance in the log term - like the case of Laura with the Vertical Shops, or Frank with the creation of Technological Parks, or John with the creation of a drone lab for the future of their campus -. Nevertheless they are willing to risk, they also claim that in those decisions, they always try to check with someone else, and they look for a minimum of information, trying to calculate risk, but as John said, "I try to calculate the risk, I am not a kamikaze, nor a hero". But when it is time to take those few fundamental decisions which shape the course of a firm, it means, that strategic decisions, are good taking (Drucker, 1967; Eisenhardt and Zbaracki, 1992). And according to interviewees, medium and high level directors must be good 
managing this uncertainty, they also have to know that those decisions are not $100 \%$ rational and that they demand the use of the left rational side of the brain, but also the right side (Mintzberg, 1976) and they must trust their gut (Hayashi, 2001); as mentioned by Jones (1999, p. 319) in Section 2.3.1.3, "in uncertain, ambiguous, or contradictory task environments, behavior is a function of goals, processing limits, and the connection between the decision maker's problem space and the task environment (objectively characterized). In this far more complex situation, problem-space representations may interact nonlinearly with goals and processing limits".

Interviewees also strengthen the importance of behavior regarding ambidexterity. They claim that exploitative activities, those that correspond to short term goals and day to day operation, are essential and they must be met in order to ensure a healthy operation. In fact, they claimed that, in general, they tend to dedicate more time to exploitation from their positions (about $65 \%$ to $80 \%$ od their time). But they also recognize the difficulty in achieving exploitation and exploration inside their teams, they agree with (Ghemawat and Costa, 1993; Tushman, et al., 2004) referred by Schulze, et al., (2008) who pointed out that "ambidexterity is associated with numerous difficulties, since the two innovation strategies, exploitation and exploration, do not only have different characteristics in terms of timeline, risk and potential return, but also call for distinct organizational structures, processes, cultures, and capabilities". Most of them, agree about a contextual type of ambidexterity, in which both exploitative and explorative activities are performed within the same team (Schulze, Heinemann, \& Abedin, 2008); regarding this, directors are aligned to what Gibson (2004, p. 209) claims, who said that in the 1970 's dual structures were recognized to be important for ambidexterity, while in the 1990's "other scholars have recognized the importance of balancing exploration and exploitation in same units trying to cope with contradictory tensions and different needs that are demanded from such different kind of activities". 
Exploration is essential in their positions, and it is not an easy think neither finding explorative directors nor doing exploration in the situation where exploitation demands the most of the time. Concerning this, they said that the main ways they use to develop exploration is by dedicating specific time to reading and being aware of external situations, in order to increase the possible creative long term projects that could provide SCA. In this same topic, they also strengthen the idea of sustaining long and deep talks and non-structured meetings with their teams focusing at the importance of being "strategic" and defining long term goals and initiatives that could separate positively from competence. Finally, in agreement to Schulze, Heinemann and Abedin (2008), directors said that they try to achieve ambidexterity alternating the two types of activities depending on the demand of the indicators or the moment of the year; there are some moments where exploitation is demanded and others where exploration; in this same line, Schulze, et al. $(2008$, p. 5) said that "senior management has to balance the interests of the exploitative and the explorative subunit in order to ensure alignment to the overall organizational goals". They also claimed that, in order to have time to explore, directors trust and delegate to other teams and their own teams those exploitative activities that do not add value of their point of view. Finally, most of the directors conclude that a strong attachment to regulations and complex centralized processes inhibit the exploration in the HEI.

Concerning the strategy and performance, we found very interesting conclusions that add value and sum important implications for managers and decision-makers in HEI, and that could be also useful for other industries. It can be concluded that the interviewee directors are aligned with the general strategy of the University. It can also be concluded that they are constantly seeking for differentiations and sources of competitive advantage, as it is expected from those positions in industries where fast changes are happening (Schulze et al., 2008; March, 1991; Porter, 2008; Barrett, 2010). The main strategies of the campus and division directors are focused in improving academic quality through having the best faculty, recruiting the most capable students, developing the best innovative educative models and trying to find that best differentiation by seizing internal and external strengths and opportunities 
in their regions (could be areas such as aeronautic, automotive, entrepreneurship, manufacturing, sustainability, among others). It was very interesting to found and corroborate that most of the directors have in their strategies a composition among autonomous or emergent - such as the Vertical Shop, the Technology Park - and deliberate or induced strategies - such as iWeek or iSemester - (Mintzberg and Waters, 1985; Burgelman, 1991). From the perspective of RBV theory and dynamic capabilities, the role of the leader consists not just in the selection of appropriated resources for the firm, but also "in appropriately adapting, integrating and reconfiguring organizational skills and resources to match changing environments" O'Reilley and Tushman (2007) when refer to (Eisenhardt and Martin, 2000; Lavie, 2006; Teece, et al., 1997;) and hence to generate sustained competitive advantage that could lead the firm to survive. In this line, we also conclude that several directors, through the constant deployment of innovative strategies, - such as innovative educative models, the improvement of faculty, the creation of technological parks, etc. - contribute to the deployment of dynamic capabilities, since they are creating constantly routines that could translate in the renewal or reconfiguration of the resources (in this case their faculty and the support areas).

Finally, how does ambidexterity and risk in decision-making affect the strategy and performance? In Section 5.2, that answer was deeply addressed using several examples that arose during the interviews with most of the directors. It is a fact that, uncertain times and complexity in competition will demand creative and innovative solutions, where decisions could not be $100 \%$ rational and where uncertainty and gut will play an important role (Gibson and Birkinshaw, 2004; Shafir, et al., 1993; Tavernier, 2005) and where bounded rationality will demand from directors to take decisions and assume consequences even when they do not have certainty in the results (Jones, 1999). In those strategic decisions, directors seem to pursue explorative actions that could provide in the future the possibility of bringing new competitive advantages to their campus or their divisions - such is the case of new Vertical Shops, the opening of new careers, the search for strategic partners, the creation of new laboratories and Technological parks, among others -; some projects 
succeeded and some did not, but directors seem to be congruent with themselves on those initiatives and they assume consequences, being aware that just the possibility of success of those big initiatives are necessary and worth the time and resources invested; like Peter said when facing a risky decision that could fail but in the case of succeed will bring positive results: "I had to make the decision, despite the risk external opinions, if I had not, then competence could surpass us". This is the way those kind of behaviors could affect performance in the organization; if Peter had not taken the risk, or if Peter had not been aware of the complex situation of HEI nowadays, then he had not made that decision, and certainly the performance of the division had been affected. More similar examples were posted in Chapter 4 and in Section 5.2.

To conclude, I wil provide an illustrative example from Laura, that shows how the faculty change its attitude towards innovative educative experiences, after two years of being wotking in such projects:

\footnotetext{
Laura: The moment I knew that all the work was worth it, was when two professors visited me and told me, "hey Laura, we are so sorry not to be involved in iWeek this year" it seems that is a great activity and most of the faculty is involved, despite they do not received any payment; but now we can sense the importance of those activities and we will be ready for next year". I was very happy about hearing that, it took me two years but now we are moving faster...
}

That example is similar to the last two in Section 5.2. They show how through "different routines" the based resource (in this case the faculty) is renewing and transforming for addressing a new and different reality. Those explorative and risky decisions could influence in the performance of an organization. Then, it is clear the way those directors decide and lead, the way they combine resources and the way they change faster than industry pace will keep them alive. In words of Balaton (2007) "capability for change becomes a synonym of efficiency and competitiveness." 


\subsection{Managerial implications}

This study has important managerial implications not just for the performance and execution of those who handle managerial positions or those who are decisionmakers, but also for the right selection and training of employees inside the firm. Next, the main managerial implications will be listed:

- Medium and high level directors, or those who are decision-makers, should be aware of the current conditions of their business, for example in the case of HEI, they should be aware about the fast changing environment and all the fast changing threats that could happen and also the opportunities that these conditions could bring (Washburn, 2005; Barrett, 2010).

- Those decision-makers or directors also must have experience and the capacity to make decisions in uncertain sceneries, and hence to take responsibilities about the consequences of their acts. As mentioned above, "strategic decision making is bounded rational in that strategic decision makers are cognitively limited and engage in a cycling among rational decision making steps" (Eisenhardt \& Zbaracki, 1992, p. 35).

- An adequate balance among explorative and exploitative activities is important for the performance of the firm (Ambrosini \& Bowman, 2009). Nevertheless, that balance does not imply a 50/50 balance; it seems, that operational activities (exploitation) demands more time than exploration. As one interviewee commented, "exploitation will pay exploration", meaning that day to day operation is necessary for having good finances in the HEI.

- Even when exploration demands less time than exploitation, interviewees agree that time devoted to exploration is much more important than the devoted in exploitation, because exploration will provide the possibilities to differentiate the organization in the future (Raisch \& Birkinshaw, 2008).

- Interviewees claim that leaders must be concerned about being competitive and working hard and constantly in trying to find sources of competitive advantages. That is the way to be ahead from competition (Peters \& Waterman Jr., 1982). 
- In Higher Education Institutions, being ambidextrous and having a positive attitude toward risk an uncertainty in decision-making is important for the performance of the institution.

- In this case, contextual ambidexterity is the most common, compared to the structural type.

- It is important to own valuable resources (VRIN resources according to the RBV theory (Wernerfelt, 1984) - but it seems to be more important the way that directors combine and use those resources for the development of competitive advantages, and at the same time, for the creation of routines that could constitute dynamic capabilities. (Ambrosini \& Bowman, 2009). As Penrose argues, the "opportunity set is also influenced by the way managers combine resources to produce productive services or capabilities" (Lockett, Thompson, \& Morgenstern, 2009, p. 14).

The above represent managerial implications, that arose from this work, and that could be useful for managers.

\subsection{Future Research}

Regarding to research on RBV and DC, it is expected to have a better understanding about those approaches in the near future. I hope more scholars and researchers spend time doing research not just in DC and RBV, but also into related topics such as knowledge management or transient advantages. And I hope they remain open not just to conventional quantitative methods, but also to qualitative and ethnographic methods that some times are more appropriate for doing RBV and DC research. As suggested by Lockett and Thompson (2001, p. 743) and quoted in Ambrosini and Bowman (2009, p. 37) "it may be necessary to sacrifice some of generality of quantitative investigation for a more qualitative attention to detail". According to Ambrosini and Bowman (2009) smaller samples are well appropriated to qualitative analysis and could be more appropriated for understanding the resource creation and regeneration process in dynamic capabilities. I am positive that those approaches are going to be significant for the competitiveness of firms in the 
increasingly changing environment. But, it is also important to keep in mind that each approach has advantages and disadvantages, and the success in achieving competitive advantage will depend on the managers, their experience and the way they combine and apply the correct strategies.

In the context of Ambidexterity, also several lines of research arises. For example, it would be advisable, to research about the specific type of ambidexterity developed in different universities, and how that type is related to the performance of different institutions. Also important is to continue with the research about the variables that promote and affect the exploration in HEI, and how that exploration is related to the development of SCA (Gibson \& Birkinshaw, 2004). In the same topic, it will be very interesting to research about possible negative effects of an excess of exploration or exploitation; for example to inquiere if "too much exploration may enhance a firm's ability to renew its knowledge base but can trap organizations in an endless cycle of search and unrewarding change" (Volberda \& Lewin, 2003) referred by (Raisch \& Birkinshaw, 2008) or if an excess of exploitation could lead to a lack of sustained competitive advantages, and hence jeopardize the future of the organization.

Research about decision-making and leadership skills constitutes a big requirement for HEI to survive and to provide better performance to society. According to Smith and Wolverton (2010, p. 68), research about leadership skills in executives working in HEI, should not be limited to senior executives; he claims that also people in other positions such as vice presidents of finance and administration, legal counsel, vice presidents of development and advancement, should be deeply survived in order to have a full understanding of those neccesary competences for effective leadership and decision-making in HEI. They also encourage scholars to do more research regarding to describing behavioral competences like empathy, sincerity, empowerment, the abilities known as "soft skills". I encourage scholars to deeply study, using quantitative and qualitative approaches, the non quantitative side of individual decision making: intuition, gut and the unconscious side of individual decision making process, which in my opinion can only be studied through empirical 
research. I also encourage scholars to address research related to the process of decision-making in HEI, and also how bounded rationality affects the decision making process. Different industries could need a different approach in decision making process, then identifying good and bad practices in different kind of industries could be very valuable for understanding such a complex topic. I think that executives who have an outstanding performance in decision-making will be more likely to represent a VRIN internal resource for their firms, and hence, provide sustained competitive advantage to their firms.

Finally, in the context of multi-campus HEI, the challenge is even bigger. The need to coordinate multiple campuses sharing a common vision and processes, but at the same time coping with such different local environments and trying to differentiate and take advantage of them, is an enormous challenge. The study about central versus descentalized way of management is also an important topic for future research. As Timberlake (2004) summarize in his multi-campus HEI study, "the hypothesis that multi-campus institutions commonly deal with tensions arising from the polarized desire for autonomy at the local level and greater control at the center of the organization. Leadership must manage the dilemma effectively in order to succeed. Leaders should establish participatory processes within the institution that provide employees with opportunities to make operational decisions locally as well as participate meaningfully in institutional strategic decision-making processes" (Timberlake, 2004, p. 98). The profile of Universities top management positions is also very interesting for research; trying to explore what characteristics are the needed ones for those managers to lead the future of HEI. 


\section{TABLES.}

\begin{tabular}{|c|c|c|}
\hline & & $\begin{array}{l}\text { Specifications } \\
\text { (Universities that offer the } \\
\text { Program/Availability of info) }\end{array}$ \\
\hline Average number of Campuses & 10 & Out of 35 Universities \\
\hline Oldest & 1929 & Out of 35 Universities \\
\hline Newest & 2003 & Out of 35 Universities \\
\hline $\begin{array}{r}\text { Average number of Bachelor } \\
\text { Degrees }\end{array}$ & 21 & Out of 35 Universities \\
\hline $\begin{array}{r}\text { Average number of Master's } \\
\text { Degrees }\end{array}$ & 15 & Out of 39 Universities \\
\hline Average number of Specializations & 12 & Out of 22 Universities \\
\hline Average number of Ph.D. Programs & 3 & Out of 23 Universities \\
\hline Average Population of Students & 20,891 & Out of 18 Universities \\
\hline Average tuition fee & 52,007 & Out of 13 Universities \\
\hline
\end{tabular}

Source: own elaboration, based on FIMPES and Universities' official webpage

Table 1. Summary of Private Multi-Campus Universities with FIMPES accreditation in México.

\begin{tabular}{|l|l|l|}
\hline \multicolumn{1}{|c}{ University } & \multicolumn{1}{c|}{$\begin{array}{c}\text { QatAm University } \\
\text { Rankings } \\
\text { Rankings }\end{array}$} \\
\hline Tecnológico de Monterrey & $\# 7$ (QS 5 Stars) & $\# 206$ \\
\hline $\begin{array}{l}\text { Instituto Tecnológico Autónomo de México } \\
\text { (ITAM) }\end{array}$ & $\# 39$ & $\# 601-650$ \\
\hline Red de Universidades Anahuac & $\# 64$ & $\# 651-700$ \\
\hline Universidad Panamericana (UP) & $\# 96$ & $\# 701+$ \\
\hline Universidad Autónoma de Guadalajara (UAG) & $\# 191-200$ & \\
\hline Red de Universidades La Salle & $\# 201-250$ & \\
\hline Universidad del Valle de México (UVM) & $\# 201-250$ & \\
\hline Universidad Tecnológica de México (UNITEC) & $\# 251-300$ & \\
\hline Universidad TecMilenio & $\# 301+$ & \\
\hline Universidad Vasco de Quiroga & $\# 301+$ & \\
\hline Universidad Interamericana para el Desarrollo & $\# 301+$ & \\
\hline
\end{tabular}

Source: own elaboration, based on QS Rankings 2016.

Table 2. Private Universities ranked according to QS Rankings 2016. 


\begin{tabular}{|c|c|c|c|}
\hline Category A & Category B & Category C & Category D \\
\hline Monterrey & Sonora Norte & Morelia & Cd. Obregón \\
\hline Guadalajara & San Luis Potosí & Cuernavaca & Irapuato \\
\hline Cd. De México & León & Culiacán & Chiapas \\
\hline Edo. De México & Chihuahua & Hidalgo & Cd. Juárez \\
\hline Querétaro & Laguna & Aguascalientes & Veracruz \\
\hline Santa Fe & & Saltillo & Zacatecas \\
\hline Puebla & & Tampico & \\
\hline Toluca & & & \\
\hline
\end{tabular}

Table 3. Categorization of the 26 campuses

of Tecnológico de Monterrey.

\begin{tabular}{|ll|}
\hline $\begin{array}{l}\text { Operational efficiency } \\
\text { Execute similar } \\
\text { activities but better } \\
\text { than rivals. }\end{array}$ & Competitive strategy \\
\hline $\begin{array}{l}\text { Extensive productivity } \\
\text { Required to obtain high }\end{array}$ & $\begin{array}{l}\text { Choose a different group of activities to bring } \\
\text { a unique combination of value. }\end{array}$ \\
\hline $\begin{array}{l}\text { It is the creation of a unique and valuable } \\
\text { position of activities that will help us define } \\
\text { which will be done and which will not. }\end{array}$ \\
\hline Source: Elaborated with data extracted from (Porter M. E., What is Strategy?, 1996) \\
\hline
\end{tabular}

Table 4. Operational Efficiency and Competitive Strategy.

\begin{tabular}{lccccc}
\multicolumn{1}{c}{ Objective } & $\begin{array}{c}\text { Campus } \\
\text { Directors }\end{array}$ & $\begin{array}{c}\text { Division } \\
\text { Directors }\end{array}$ & Rector & $\begin{array}{c}\text { Campus Vice- } \\
\text { president }\end{array}$ & Total \\
\hline In-depth & 4 & 8 & 0 & 0 & 12 \\
\hline Confirmatory & 1 & 0 & 1 & 1 & 3 \\
\hline & 5 & 8 & 1 & 1 & 15 \\
\hline
\end{tabular}

Table 5A. In-depth and Confirmatory Interviewees. 


\begin{tabular}{|c|c|c|c|c|c|}
\hline \multirow[b]{2}{*}{ Participants } & \multicolumn{2}{|c|}{ Campus Category } & \multirow[b]{2}{*}{$\begin{array}{c}\text { Total } \\
\text { Sample }\end{array}$} & \multicolumn{2}{|c|}{ Confirmatory interview } \\
\hline & $\begin{array}{c}\text { Campus } \\
\text { A }\end{array}$ & $\begin{array}{c}\text { Campus } \\
\text { B }\end{array}$ & & $\begin{array}{c}\text { Rector } \\
\text { and Vice- } \\
\text { President }\end{array}$ & $\begin{array}{l}\text { Campus } \\
\text { Director }\end{array}$ \\
\hline Campus director & 1 & 3 & 4 & 2 & 1 \\
\hline Director of Division & 2 & 6 & 8 & & \\
\hline Total Sample & 3 & 9 & 12 & & 3 \\
\hline
\end{tabular}

Table 5B. Sample Selection of the In-depth and Confirmatory Interviews (According to Category of Campus).

\begin{tabular}{ll}
\hline Area & Questions \\
\hline Strategy & Tell me briefly about the process of strategic planning that you execute? \\
What members of your team are involved in this process? \\
Tell me about the content of your strategy in the last year? On what is your strategy \\
focused? What are the differentiators of your division/campus and how do you \\
achieve them? \\
How do you perceive the strategy of the University? How long time will take the \\
strategy to be implemented? \\
Tell me about your KPI, Are they more short term or long term? Please tell me \\
examples. \\
Is your strategy aligned to the University? How is it? \\
Do you consider yourself more short term or long term strategist? Why? Give me \\
examples. \\
What percentage of your KPI belongs to explorative and what percentage to \\
exploitative? \\
Can you mention in the last seasons, the most important innovations or advantages \\
that you have developed? \\
Considering your skills, are you more exploitative or explorative? Please give me \\
examples of actions that will create a difference in your campus/division? How \\
often do you propose those kinds of explorative or long term activities? Please give \\
examples. \\
What KPI belongs to those long term or explorative activities? What percentage of \\
your time is for exploration and what for exploitation? Why? \\
In what extent do you consider yourself good for balancing the two kinds of \\
activities? What do you do in order to have the time to develop both, especially \\
exploration? How do you make your time and yourself to focus in short term, but at \\
the same time focused in the long term? How your boss and the institutions \\
support you for doing exploration and exploitation? How do your team behave \\
regarding to exploitation and exploration? How good are they for each type of \\
activities? \\
Do you consider that high level positions versus low level positions should have a \\
different composition regarding to the percentage of time devoted to exploration \\
versus exploitation? Why? \\
In your point of view, is it more difficult to find explorative or exploitative profiles \\
in decision-makers? Why?
\end{tabular}


Decisionmaking

General questions involving various themes
Explain me please deeply, How do you do with your team to deal with those day to day activities that must be accomplished and at the same time dealing with those really important activities that are more innovative and that will differentiate your programs?

But there are those kind of decisions that will transform your division or that the result will impact strongly good or strongly bad. Related to that kind of decisions How often do you take those kinds of decisions? Can you remember and mention some examples.

Regarding to this kind of strategic decisions (for example when opening a new minor or when hiring a new director or when opening or closing a career) how do you behave related to the decision-making process, I mean, are you a fast decision taker, do you evaluate rapidly the situation and trust in your gut or are you the kind of person that think very well the situation and evaluate all the different sceneries and consequences until you confident sure about the decision? Can you give us examples?

Regarding to these kinds of complex decisions, how do you consider that works the best, taking rapidly and trusting in your gut and experience regardless you do not have all the information or the other way having the maximum quantity of information? What happens when you do not have enough information? Are you prone to take decisions or do you prefer to wait? Can you please exemplify with one or two examples?

Are you a person who used to take risks or fast decisions on those important decisions that can create a big difference in your division? Tell me an example of this kind of decision. How often do you take those decisions? According to your response: do you consider yourself as a risky person or as a cautious one? What do you think that is better for an enterprise?

Finally, regarding to this level of decisions and the performance of your divisions, please tell me how strong are these kinds of decisions with the performance and the growth of your division?

How autonomous are you when taking decisions? Do you have the support of your boss or do you always need to have his approval?

When you take risky decisions or those where you do not have certainty of success, is failure an important variable to avoid those decisions? What happens if you fail? What is your attitude when failing? Can you give me examples?

Please, tell me one big project where you have failed? How important was the support from your boss? In general, how do you consider the posture and culture of the institutions regarding to supporting when making mistakes?

Are you supportive with your team when making mistakes? How important is that in positions like yours?

Regarding to flexibility, do you consider it important on the performance of your team? Can you mention some examples? What about rigidity, in which conditions is it good? Are your directors flexible about regulations?

How important is risk and uncertainty in decision-making on high level positions when compared to low level?

Next question is very important: When you address very difficult decisions, for example when you are asked to open a new career, in general, can you describe the process that you follow in order to decide whether open or not open that career? Can you deeply explain?

Talking about your leaders, I want you to tell me how prone are they to appropriately adapting, integrating and reconfiguring organizational skills and resources to match changing environments? Please enunciate some specific examples? In the last years tell me please, How have you done to overcome complex problems 
for example to rescue those careers that are about to close? Or to reach that goal that no one had reached? Do you think that all that you told me is kind of a capability or kind of a habit? Tell me please why.

Source: Elaborated by author, considering that this is a semi-structured interview and that questions are a guideline.

Table 6. Guide of Questions for the in-depth semi-structured interview.

\begin{tabular}{|c|c|}
\hline Area & Questions \\
\hline Strategy & $\begin{array}{l}\text { Tell me briefly about the process of strategic planning that you execute? } \\
\text { How do you perceive the strategy of the University? How long time will take the } \\
\text { strategy to be implemented? } \\
\text { Tell me about your KPI, Are they more short term or long term? Please tell me } \\
\text { examples. }\end{array}$ \\
\hline Ambidexterity & $\begin{array}{l}\text { Do you consider yourself more short term or long term strategist? Why? Give me } \\
\text { examples. } \\
\text { What KPI belongs to those long term or explorative activities? What percentage of } \\
\text { your time is for exploration and what for exploitation? Why? } \\
\text { In what extent do you consider yourself good for balancing the two kinds of } \\
\text { activities? What do you do in order to have the time to develop both, especially } \\
\text { exploration? How do you make your time and yourself to focus in short term, but at } \\
\text { the same time focused in the long term? } \\
\text { Do you consider that high level positions versus low level positions should have a } \\
\text { different composition regarding to the percentage of time devoted to exploration } \\
\text { versus exploitation? Why? } \\
\text { In your point of view, is it more difficult to find explorative or exploitative profiles } \\
\text { in decision-makers? Why? }\end{array}$ \\
\hline $\begin{array}{l}\text { Decision- } \\
\text { making }\end{array}$ & $\begin{array}{l}\text { But there are those kind of decisions that will transform your division or that the } \\
\text { result will impact strongly good or strongly bad. Related to that kind of decisions } \\
\text { How often do you take those kinds of decisions? Can you remember and mention } \\
\text { some examples. } \\
\text { Regarding to this kind of strategic decisions (for example when opening a new } \\
\text { minor or when hiring a new director or when opening or closing a career) how do } \\
\text { you behave related to the decision-making process, I mean, are you a fast decision } \\
\text { taker, do you evaluate rapidly the situation and trust in your gut or are you the } \\
\text { kind of person that think very well the situation and evaluate all the different } \\
\text { sceneries and consequences until you confident sure about the decision? Can you } \\
\text { give us examples? } \\
\text { When you take risky decisions or those where you do not have certainty of success, } \\
\text { is failure an important variable to avoid those decisions? What happens if you fail? } \\
\text { What is your attitude when failing? Can you give me examples? } \\
\text { Please, tell me one big project where you have failed? How important was the } \\
\text { support from your boss? In general, how do you consider the posture and culture } \\
\text { of the institutions regarding to supporting when making mistakes? } \\
\text { How important is risk and uncertainty in decision-making on high level positions } \\
\text { when compared to low level? }\end{array}$ \\
\hline
\end{tabular}


General questions involving various themes
Next question is very important: When you address very difficult decisions, for example when you are asked to open a new career, in general, can you describe the process that you follow in order to decide whether open or not open that career? Can you deeply explain?

In the last years tell me please, How have you done to overcome complex problems for example to rescue those careers that are about to close? Or to reach that goal that no one had reached? Do you think that all that you told me is kind of a capability or kind of a habit? Tell me please why.

Source: Elaborated by author, considering that this is a semi-structured interview and that questions are a guideline.

Table 7. Guide of Questions for the confirmatory semi-structured interview.

\section{Type of} Interview
In-depth In-depth In-depth In-depth In-depth In-depth In-depth In-depth In-depth In-depth In-depth In-depth Confirmatory Confirmatory

Confirmatory

\section{Date} May, 25th

May, 25th
June, 15th April, 22th May, 19th May, 26th May, 26th June, 16th April, 22th April, 21th May, 19th

May, 19th
May, 19th June, 27th June, 15th

\section{Place of Length of Interview Recorded Interview (HH:MM:SS)}

Size in Words

Meeting Room

Yes

1:38:36

13,485

His office

Yes

$0: 46: 46$

6,928

His office

Yes

$1: 10: 57$

9,326

His office

Yes

$0: 52: 52$

7,198

His office

Yes

$1: 15: 50$

His office

Yes

1:12:24

6,504

Yes $\quad 1: 05: 59 \quad 9,571$

Meeting Room

Yes

0:56:23

8,999

His office

Yes

His office
Meeting Room

Yes

1:10:44

8,215

$0: 57: 59$

8,687

Mary

Ross

$\begin{array}{cc}\text { Meeting Room } & \text { Yes } \\ \text { His house } & \text { No } \\ \text { His office } & \text { Yes } \\ \text { His office } & \text { Yes }\end{array}$

$\begin{array}{lll}\text { Yes } & 1: 19: 43 & 10,718 \\ \text { Yes } & 1: 27: 14 & 10,272\end{array}$

$\begin{array}{ccc} & 1: 27: 14 & 10,272 \\ \text { es } & 1: 54: 13 & \text { NA }\end{array}$

$\begin{array}{lll}\text { Yes } & 0: 34: 01 & \text { NA } \\ \text { Yes } & & 5,195\end{array}$

\begin{tabular}{lll} 
Yes & $0: 26: 54$ & 4,567 \\
\hline
\end{tabular}

Table 8. General data from the interviews.

\begin{tabular}{|c|l|c|c|c|}
\hline$\#$ & \multicolumn{1}{c|}{ Code } & $\begin{array}{c}\text { Extended } \\
\text { Codes }\end{array}$ & Mentions & $\begin{array}{c}\text { Percentage of } \\
\text { Mentions }\end{array}$ \\
\hline $\mathbf{A}$ & Risk and Uncertainty in Decision-Making & $\mathbf{7 6}$ & $\mathbf{2 6 7}$ & $\mathbf{2 8 \%}$ \\
\hline $\mathbf{1}$ & DM Fast Theme & 10 & $\mathbf{2 9}$ & $\mathbf{3 \%}$ \\
$\mathbf{2}$ & DM Risk Boss and High Directors & 13 & $\mathbf{4 7}$ & $\mathbf{5 \%}$ \\
$\mathbf{3}$ & DM Risk Gut & 11 & $\mathbf{4 8}$ & $\mathbf{5 \%}$ \\
$\mathbf{4}$ & DM Risk High & 8 & $\mathbf{3 2}$ & $\mathbf{3 \%}$ \\
\hline
\end{tabular}




\begin{tabular}{|clccc|}
\hline $\mathbf{5}$ & DM Risk Low & 3 & $\mathbf{6}$ & $\mathbf{1 \%}$ \\
$\mathbf{6}$ & DM Risk Medium & 8 & $\mathbf{3 4}$ & $\mathbf{4 \%}$ \\
$\mathbf{7}$ & DM Risk Mistake & 5 & $\mathbf{1 9}$ & $\mathbf{2 \%}$ \\
$\mathbf{8}$ & DM Risk need to chek & 6 & $\mathbf{3 0}$ & $\mathbf{3 \%}$ \\
$\mathbf{9}$ & DM Risk Team & 7 & $\mathbf{1 4}$ & $\mathbf{1 \%}$ \\
$\mathbf{1 0}$ & DM Style & 5 & $\mathbf{8}$ & $\mathbf{1 \%}$ \\
\hline $\mathbf{B}$ & Ambidexterity & $\mathbf{9 5}$ & $\mathbf{2 8 1}$ & $\mathbf{3 0 \%}$ \\
\hline $\mathbf{1 1}$ & Ambidexterity Interviewed & 13 & $\mathbf{3 4}$ & $\mathbf{4 \%}$ \\
\hline $\mathbf{1 2}$ & Ambidexterity Successor & 9 & $\mathbf{1 2}$ & $\mathbf{1 \%}$ \\
\hline $\mathbf{1 3}$ & Ambidexterity High Directors & 9 & $\mathbf{3 4}$ & $\mathbf{4 \%}$ \\
\hline $\mathbf{1 4}$ & Ambidexterity Team & 13 & $\mathbf{2 2}$ & $\mathbf{2 \%}$ \\
\hline $\mathbf{1 5}$ & Ambidexterity How Happens & 17 & $\mathbf{6 6}$ & $\mathbf{7 \%}$ \\
\hline $\mathbf{1 6}$ & Ambidexterity Structural & 4 & $\mathbf{6}$ & $\mathbf{1 \%}$ \\
\hline $\mathbf{1 7}$ & Ambidexterity Contextual & 13 & $\mathbf{3 4}$ & $\mathbf{4 \%}$ \\
\hline $\mathbf{1 8}$ & Ambidexterity Threats & 7 & $\mathbf{2 2}$ & $\mathbf{2 \%}$ \\
\hline $\mathbf{1 9}$ & Ambidexterity General & 5 & $\mathbf{4 2}$ & $\mathbf{4 \%}$ \\
\hline $\mathbf{2 0}$ & Ambidexterity Boss & 5 & $\mathbf{9}$ & $\mathbf{1 \%}$ \\
\hline $\mathbf{C}$ & Strategy & $\mathbf{1 5 5}$ & $\mathbf{3 9 9}$ & $\mathbf{4 2 \%}$ \\
\hline $\mathbf{2 1}$ & Strategy Alignment & 3 & $\mathbf{2 9}$ & $\mathbf{3 \%}$ \\
\hline $\mathbf{2 2}$ & Strategy Boss & 3 & $\mathbf{1 6}$ & $\mathbf{2 \%}$ \\
\hline $\mathbf{2 3}$ & Strategy Interviewed & 62 & $\mathbf{1 7 2}$ & $\mathbf{1 8 \%}$ \\
\hline $\mathbf{2 4}$ & Strategy Interviewed Differentiation & 21 & $\mathbf{5 3}$ & $\mathbf{6 \%}$ \\
\hline $\mathbf{2 5}$ & Strategy Planning & 3 & $\mathbf{9}$ & $\mathbf{1 \%}$ \\
\hline $\mathbf{2 6}$ & Strategy TEC Negative Issues & 13 & $\mathbf{2 5}$ & $\mathbf{3 \%}$ \\
\hline $\mathbf{2 7}$ & Strategy Possible Threats & 18 & $\mathbf{2 6}$ & $\mathbf{3 \%}$ \\
\hline $\mathbf{2 8}$ & Strategy TEC Time Term & 4 & $\mathbf{7}$ & $\mathbf{1 \%}$ \\
\hline $\mathbf{2 9}$ & Strategy TEC & $\mathbf{6 2}$ & $\mathbf{7 \%}$ \\
\hline & TOTALS: & $\mathbf{9 4 7}$ & $\mathbf{1 0 0 \%}$ \\
\hline & & & & \\
\hline
\end{tabular}

Table 9. General Codes and Themes.

\begin{tabular}{|c|c|c|c|c|c|c|c|c|c|c|c|c|c|c|c|}
\hline$\#$ & Reduced Code & $\frac{0}{3}$ & 商 & $\stackrel{8}{\Xi}$ & 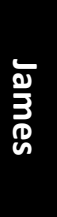 & 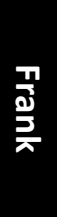 & 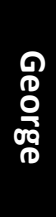 & $\begin{array}{l}\frac{7}{0} \\
\frac{0}{10} \\
\frac{7}{7}\end{array}$ & ֻ & চ্চ & $\frac{\text { Do }}{\frac{8}{9}}$ & $\stackrel{\mathscr{E}}{\underline{E}}$ & 羕 & $\begin{array}{l}\frac{z}{3} \\
\frac{1}{z} \\
\frac{1}{0} \\
\frac{z}{4}\end{array}$ & 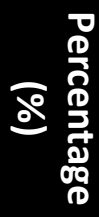 \\
\hline A & DM Fast Theme & 2 & 2 & 3 & 4 & 5 & 0 & 2 & 0 & 1 & 8 & 1 & 1 & 29 & 11 \\
\hline B & $\begin{array}{r}\text { DM Risk Boss and } \\
\text { High Directors }\end{array}$ & 6 & 0 & 1 & 6 & 9 & 3 & 2 & 2 & 4 & 8 & 2 & 4 & 47 & 18 \\
\hline C & DM Risk Gut & 5 & 2 & 11 & 6 & 2 & 0 & 0 & 7 & 3 & 5 & 3 & 4 & 48 & 18 \\
\hline
\end{tabular}




\begin{tabular}{|c|r|c|c|c|c|c|c|c|c|c|c|c|c|c|c|}
\hline D & DM Risk High & 8 & 0 & 0 & 7 & 1 & 1 & 3 & 0 & 0 & 10 & 1 & 1 & $\mathbf{3 2}$ & $\mathbf{1 2}$ \\
\hline E & DM Risk Low & 0 & 4 & 0 & 0 & 0 & 1 & 0 & 0 & 0 & 0 & 1 & 0 & $\mathbf{6}$ & $\mathbf{2}$ \\
\hline F & DM Risk Medium & 2 & 1 & 1 & 0 & 5 & 2 & 1 & 3 & 2 & 6 & 5 & 6 & $\mathbf{3 4}$ & $\mathbf{1 3}$ \\
\hline G & DM Risk Mistake & 4 & 0 & 0 & 3 & 2 & 1 & 0 & 0 & 1 & 5 & 2 & 1 & $\mathbf{1 9}$ & $\mathbf{7}$ \\
\hline H & $\begin{array}{r}\text { DM Risk need to } \\
\text { chek }\end{array}$ & 3 & 3 & 3 & 4 & 1 & 1 & 1 & 0 & 3 & 1 & 2 & 8 & $\mathbf{3 0}$ & $\mathbf{1 1}$ \\
\hline I & DM Risk Team & 2 & 0 & 0 & 0 & 1 & 2 & 0 & 2 & 4 & 0 & 1 & 2 & $\mathbf{1 4}$ & $\mathbf{5}$ \\
\hline J & DM Style & 2 & 0 & 0 & 0 & 0 & 2 & 1 & 0 & 1 & 0 & 2 & 0 & $\mathbf{8}$ & $\mathbf{3}$ \\
\hline & TOTALS: & $\mathbf{3 4}$ & $\mathbf{1 2}$ & $\mathbf{1 9}$ & $\mathbf{3 0}$ & $\mathbf{2 6}$ & $\mathbf{1 3}$ & $\mathbf{1 0}$ & $\mathbf{1 4}$ & $\mathbf{1 9}$ & $\mathbf{4 3}$ & $\mathbf{2 0}$ & $\mathbf{2 7}$ & $\mathbf{2 6 7}$ & $\mathbf{1 0 0}$ \\
\hline
\end{tabular}

Table 10. Codes, Mentions and Percentage of Mentions for

Risk and Uncertainty in DM Theme at Interviewee detailed.

\begin{tabular}{|c|c|c|c|}
\hline$\#$ & Reduced Code & Mentions & Percentage \\
\hline$A$ & DM Fast Theme & 29 & $11 \%$ \\
\hline B & DM Risk Boss and High Directors & 47 & $18 \%$ \\
\hline C & DM Risk Gut & 48 & $18 \%$ \\
\hline D & DM Risk High & 32 & $12 \%$ \\
\hline $\mathrm{E}$ & DM Risk Low & 6 & $2 \%$ \\
\hline $\mathrm{F}$ & DM Risk Medium & 34 & $13 \%$ \\
\hline G & DM Risk Mistake & 19 & $7 \%$ \\
\hline $\mathrm{H}$ & DM Risk need to chek & 30 & $11 \%$ \\
\hline I & DM Risk Team & 14 & $5 \%$ \\
\hline $\mathrm{J}$ & DM Style & 8 & $3 \%$ \\
\hline & TOTALS: & 267 & $100 \%$ \\
\hline
\end{tabular}

Table 11. Codes, Mentions and Percentage of Mentions for Risk and Uncertainty in DM Theme.

\begin{tabular}{|c|c|c|c|c|c|c|c|c|c|c|c|c|c|c|}
\hline$\#$ & CODE & $\frac{\text { 울 }}{3}$ & 公 & ฏ & 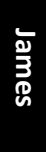 & 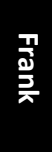 & 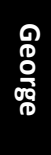 & $\begin{array}{l}\frac{7}{8} \\
\frac{0}{7} \\
\frac{1}{7}\end{array}$ & $\frac{5}{5}$ & চ্ণ & $\begin{array}{l}\text { D } \\
\stackrel{\mathbb{D}}{\mathbb{D}}\end{array}$ & $\stackrel{\mathscr{a}}{\underline{E}}$ & $\frac{3}{2}$ & 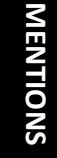 \\
\hline A & DM Fast Theme & 2 & 2 & 3 & 4 & 5 & 0 & 2 & 0 & 1 & 7 & 1 & 1 & 29 \\
\hline A1 & $\begin{array}{l}\text { Boss DM Like Bosses that are Fast } \\
\text { for DM }\end{array}$ & 0 & 0 & 0 & 0 & 2 & 0 & 0 & 0 & 0 & 0 & 0 & 0 & 2 \\
\hline $\mathrm{A} 2$ & $\begin{array}{l}\text { DM Being Attached to the Rules } \\
\text { Makes you Slower in DM }\end{array}$ & 0 & 0 & 1 & 2 & 0 & 0 & 0 & 0 & 0 & C & 0 & 0 & 4 \\
\hline
\end{tabular}




\begin{tabular}{|c|c|c|c|c|c|c|c|c|c|c|c|c|c|c|}
\hline A3 & $\begin{array}{l}\text { DM Employeers want Fast DM } \\
\text { workers }\end{array}$ & 0 & 0 & 0 & 0 & 1 & 0 & 0 & 0 & 0 & 0 & 0 & 0 & 1 \\
\hline A4 & DM Fast taking Decisions & 0 & 0 & 0 & 0 & 0 & 0 & 1 & 0 & 0 & 3 & 0 & 0 & 4 \\
\hline A5 & $\begin{array}{l}\text { DM HIGH DIRECTORS in TEC are } \\
\text { Fast because the tight Competence }\end{array}$ & 0 & 0 & 0 & 0 & 0 & 0 & 0 & 0 & 0 & 2 & 0 & 0 & 2 \\
\hline A6 & DM Mexicans are slow in DM & 0 & 0 & 0 & 0 & 1 & 0 & 0 & 0 & 0 & 0 & 0 & 0 & 1 \\
\hline A7 & $\begin{array}{l}\text { DM Risk better to apologize but do } \\
\text { it }\end{array}$ & 2 & 0 & 0 & 1 & 1 & 0 & 1 & 0 & 0 & 2 & 0 & 0 & 7 \\
\hline A8 & DM Risk Gut Fast When Sure & 0 & 0 & 1 & 0 & 0 & 0 & 0 & 0 & 1 & 0 & 1 & 1 & 4 \\
\hline A9 & $\begin{array}{l}\text { DM Risk GUT NO Like Boss } \\
\text { Attached to Regulations } 100 \%\end{array}$ & 0 & 0 & 1 & 1 & 0 & 0 & 0 & 0 & 0 & 0 & 0 & 0 & 2 \\
\hline A10 & $\begin{array}{l}\text { DM Risk just when } 100 \% \text { Secure it } \\
\text { will not affect TEC Rules, low Risk }\end{array}$ & 0 & 2 & 0 & 0 & 0 & 0 & 0 & 0 & 0 & 0 & 0 & 0 & 2 \\
\hline B & DM Risk Boss and High Directors & 6 & $\mathbf{0}$ & 1 & 6 & 9 & 3 & 2 & 2 & 4 & 8 & 2 & 4 & 47 \\
\hline B1 & $\begin{array}{l}\text { Boss DM Like Bosses that are Fast } \\
\text { for DM }\end{array}$ & 0 & 0 & 0 & 0 & 2 & 0 & 0 & 0 & 0 & 0 & 0 & 0 & 2 \\
\hline B2 & Boss DM Risk Support for Mistake & 1 & 0 & 0 & 1 & 1 & 1 & 0 & 0 & 1 & 1 & 0 & 0 & 6 \\
\hline B3 & Boss DM Risk Support for Risk & 1 & 0 & 0 & 2 & 1 & 1 & 0 & 0 & 1 & 0 & 1 & 1 & 8 \\
\hline B4 & DM Risk Boss & 1 & 0 & 0 & 0 & 1 & 0 & 1 & 1 & 0 & 2 & 1 & 1 & 8 \\
\hline B5 & $\begin{array}{l}\text { DM Risk Boss Aligned when visions } \\
\text { are aligned }\end{array}$ & 0 & 0 & 0 & 0 & 1 & 1 & 0 & 0 & 0 & 0 & 0 & 0 & 2 \\
\hline B6 & DM Risk Boss Low Risk & 0 & 0 & 0 & 0 & 1 & 0 & 1 & 1 & 0 & 3 & 0 & 0 & 6 \\
\hline B7 & $\begin{array}{l}\text { DM Risk Boss not Aligned not } \\
\text { Support }\end{array}$ & 0 & 0 & 0 & 0 & 0 & 0 & 0 & 0 & 0 & 1 & 0 & 0 & 1 \\
\hline B8 & DM Risk Boss Very High & 1 & 0 & 0 & 1 & 0 & 0 & 0 & 0 & 0 & 0 & 0 & 1 & 3 \\
\hline B9 & $\begin{array}{l}\text { DM Risk GUT NO Like Boss } \\
\text { Attached to Regulations } 100 \%\end{array}$ & 0 & 0 & 1 & 1 & 0 & 0 & 0 & 0 & 0 & 0 & 0 & 0 & 2 \\
\hline B10 & $\begin{array}{l}\text { DM Risk Important in HIGH } \\
\text { DIRECTORS }\end{array}$ & 0 & 0 & 0 & 0 & 1 & 0 & 0 & 0 & 0 & 0 & 0 & 0 & 1 \\
\hline B11 & $\begin{array}{l}\text { DM Risk Mistake non Support from } \\
\text { Boss }\end{array}$ & 0 & 0 & 0 & 0 & 0 & 0 & 0 & 0 & 0 & 1 & 0 & 0 & 1 \\
\hline B12 & $\begin{array}{l}\text { DM Risk Reduce learning from } \\
\text { Experience }\end{array}$ & 0 & 0 & 0 & 1 & 0 & 0 & 0 & 0 & 1 & 0 & 0 & 0 & 2 \\
\hline B13 & $\begin{array}{l}\text { Team Successor Selected more } \\
\text { Manage Risk (HIGH DIRECTOR) }\end{array}$ & 2 & 0 & 0 & 0 & 1 & 0 & 0 & 0 & 1 & 0 & 0 & 1 & 5 \\
\hline C & DM Risk Gut & 5 & 2 & 11 & 6 & 2 & $\mathbf{0}$ & 0 & 7 & 3 & 5 & 3 & 4 & 48 \\
\hline $\mathrm{C} 1$ & DM Risk Gut based on KPI & 1 & 0 & 0 & 0 & 0 & 0 & 0 & 0 & 0 & 0 & 0 & 0 & 1 \\
\hline $\mathrm{C} 2$ & DM Risk Gut Example & 0 & 0 & 3 & 0 & 0 & 0 & 0 & 1 & 0 & 1 & 0 & 1 & 6 \\
\hline $\mathrm{C} 3$ & DM Risk Gut Fast When Sure & 0 & 0 & 1 & 0 & 0 & 0 & 0 & 0 & 1 & 0 & 1 & 1 & 4 \\
\hline C4 & $\begin{array}{l}\text { DM Risk Gut He is not Clear } \\
\text { Confuse Response }\end{array}$ & 0 & 1 & 0 & 0 & 0 & 0 & 0 & 0 & 0 & 0 & 0 & 0 & 1 \\
\hline $\mathrm{C} 5$ & DM Risk Gut Important in DM & 2 & 0 & 2 & 2 & 1 & 0 & 0 & 1 & 0 & 2 & 1 & 1 & 12 \\
\hline C6 & $\begin{array}{l}\text { DM Risk Gut Important in HIGH } \\
\text { DIRECTORS }\end{array}$ & 0 & 0 & 1 & 2 & 1 & 0 & 0 & 1 & 1 & 1 & 0 & 0 & 7 \\
\hline $\mathrm{C7}$ & $\begin{array}{l}\text { DM Risk Gut is Because you are not } \\
\text { Expert in Everything (confuse } \\
\text { answer) }\end{array}$ & 0 & 1 & 0 & 0 & 0 & 0 & 0 & 0 & 0 & 0 & 0 & 0 & 1 \\
\hline $\mathrm{C} 8$ & DM Risk Gut is Pattern (AGREGAR) & 0 & 0 & 0 & 1 & 0 & 0 & 0 & 0 & 0 & 0 & 0 & 0 & 1 \\
\hline C9 & DM Risk Gut Medium & 0 & 0 & 2 & 0 & 0 & 0 & 0 & 4 & 1 & 1 & 1 & 0 & 9 \\
\hline C10 & $\begin{array}{l}\text { DM Risk GUT NO Like Boss } \\
\text { Attached to Regulations } 100 \%\end{array}$ & 0 & 0 & 1 & 1 & 0 & 0 & 0 & 0 & 0 & 0 & 0 & 0 & 2 \\
\hline C11 & $\begin{array}{l}\text { DM Risk Gut use after Validation } \\
\text { No DM alone }\end{array}$ & 2 & 0 & 1 & 0 & 0 & 0 & 0 & 0 & 0 & 0 & 0 & 1 & 4 \\
\hline D & DM Risk High & 8 & $\mathbf{0}$ & 0 & 7 & 1 & 1 & 3 & 0 & $\mathbf{0}$ & 10 & 1 & 1 & 32 \\
\hline D1 & $\begin{array}{l}\text { DM HIGH DIRECTORS in TEC are } \\
\text { Fast because the tight Competence }\end{array}$ & 0 & 0 & 0 & 0 & 0 & 0 & 0 & 0 & 0 & 2 & 0 & 0 & 2 \\
\hline
\end{tabular}




\begin{tabular}{|c|c|c|c|c|c|c|c|c|c|c|c|c|c|c|}
\hline D2 & $\begin{array}{l}\text { DM Risk better to apologize but do } \\
\text { it }\end{array}$ & 2 & 0 & 0 & 1 & 1 & 0 & 1 & 0 & 0 & 2 & 0 & 0 & 7 \\
\hline D3 & DM Risk Boss Very High & 1 & 0 & 0 & 1 & 0 & 0 & 0 & 0 & 0 & 0 & 0 & 1 & 3 \\
\hline D4 & DM Risk High & 3 & 0 & 0 & 2 & 0 & 0 & 0 & & 0 & 2 & 0 & 0 & 7 \\
\hline D5 & DM Risk High Example & 1 & 0 & 0 & 2 & 0 & 0 & 1 & 0 & 0 & 2 & 1 & 0 & 7 \\
\hline D6 & $\begin{array}{l}\text { DM Risk take Decision it does not } \\
\text { Matter if there is a Price to Pay }\end{array}$ & 1 & 0 & 0 & 1 & 0 & 0 & 1 & 0 & 0 & 0 & 0 & 0 & 3 \\
\hline D7 & $\begin{array}{l}\text { DM Risk take it because } \\
\text { Competence is Not Seat and Static }\end{array}$ & 0 & 0 & 0 & 0 & 0 & 0 & 0 & 0 & 0 & 2 & 0 & 0 & 2 \\
\hline D8 & $\begin{array}{l}\text { DM Risk there is a Culture for } \\
\text { Taking Risk }\end{array}$ & 0 & 0 & 0 & 0 & 0 & 1 & 0 & 0 & 0 & 0 & 0 & 0 & 1 \\
\hline $\mathbf{E}$ & DM Risk Low & $\mathbf{0}$ & 4 & $\mathbf{0}$ & $\mathbf{0}$ & $\mathbf{0}$ & 1 & $\mathbf{0}$ & $\mathbf{0}$ & 0 & $\mathbf{0}$ & 1 & $\mathbf{0}$ & 6 \\
\hline E1 & $\begin{array}{l}\text { DM Risk just when } 100 \% \text { Secure it } \\
\text { will not affect TEC Rules, low Risk }\end{array}$ & 0 & 2 & 0 & 0 & 0 & 0 & 0 & 0 & 0 & 0 & 0 & 0 & 2 \\
\hline E2 & DM Risk Low & 0 & 1 & 0 & 0 & 0 & 0 & 0 & 0 & 0 & 0 & 0 & 0 & 1 \\
\hline E3 & DM Risk When Sure & 0 & 1 & 0 & 0 & 0 & 1 & 0 & 0 & 0 & 0 & 1 & 0 & 3 \\
\hline $\mathbf{F}$ & DM Risk Medium & 2 & 1 & 1 & $\mathbf{0}$ & 5 & 2 & 1 & 3 & 2 & 6 & 5 & 6 & 34 \\
\hline $\mathrm{F} 1$ & DM Mexicans are slow in DM & 0 & 0 & 0 & 0 & 1 & 0 & 0 & 0 & 0 & 0 & 0 & 0 & 1 \\
\hline F2 & DM Risk Medium & 1 & 0 & 0 & 0 & 0 & 0 & 1 & 2 & 0 & 3 & 2 & 0 & 9 \\
\hline F3 & DM Risk Medium Example & 1 & 0 & 0 & 0 & 0 & 0 & 0 & 1 & 1 & 3 & 1 & 2 & 9 \\
\hline F4 & DM Risk Medium to Low & 0 & 0 & 1 & 0 & 1 & 1 & 0 & 0 & 1 & 0 & 0 & 3 & 7 \\
\hline F5 & DM Risk Medium to Low Example & 0 & 0 & 0 & 0 & 1 & 0 & 0 & 0 & 0 & 0 & 0 & 1 & 2 \\
\hline F6 & $\begin{array}{l}\text { DM Risk Mexicans are afraid of DM } \\
\text { for fear }\end{array}$ & 0 & 0 & 0 & 0 & 1 & 0 & 0 & 0 & 0 & 0 & 0 & 0 & 1 \\
\hline F7 & DM Risk When No Vital Decisions & 0 & 0 & 0 & 0 & 1 & 0 & 0 & 0 & 0 & 0 & 1 & 0 & 2 \\
\hline F8 & DM Risk When Sure & 0 & 1 & 0 & 0 & 0 & 1 & 0 & 0 & 0 & 0 & 1 & 0 & 3 \\
\hline G & DM Risk Mistake & 4 & $\mathbf{0}$ & $\mathbf{0}$ & 3 & 2 & 1 & $\mathbf{0}$ & $\mathbf{0}$ & 1 & 5 & 2 & 1 & 19 \\
\hline G1 & Boss DM Risk Support for Mistake & 1 & 0 & 0 & 1 & 1 & 1 & 0 & 0 & 1 & 1 & 0 & 0 & 6 \\
\hline $\mathrm{G} 2$ & $\begin{array}{l}\text { DM Risk Mistake Its Ok But Not } \\
\text { Repeat Same Error }\end{array}$ & 1 & 0 & 0 & 1 & 1 & 0 & 0 & 0 & 0 & 3 & 1 & 0 & 7 \\
\hline G3 & $\begin{array}{l}\text { DM Risk Mistake No Problem but } \\
\text { Never Hide Information (thrust) }\end{array}$ & 1 & 0 & 0 & 0 & 0 & 0 & 0 & 0 & 0 & 0 & 0 & 0 & 1 \\
\hline G4 & $\begin{array}{l}\text { DM Risk Mistake non Support from } \\
\text { Boss }\end{array}$ & 0 & 0 & 0 & 0 & 0 & 0 & 0 & 0 & 0 & 1 & 0 & 0 & 1 \\
\hline G5 & $\begin{array}{l}\text { DM Risk Mistake Support when } \\
\text { Happens }\end{array}$ & 1 & 0 & 0 & 1 & 0 & 0 & 0 & 0 & 0 & 0 & 1 & 1 & 4 \\
\hline H & DM Risk need to chek & 3 & 3 & 3 & 4 & 1 & 1 & 1 & $\mathbf{0}$ & 3 & 1 & 2 & 8 & 30 \\
\hline $\mathrm{H} 1$ & $\begin{array}{l}\text { DM Being Attached to the Rules } \\
\text { Makes you Slower in DM }\end{array}$ & 0 & 0 & 1 & 2 & 0 & 0 & 0 & 0 & 0 & 1 & 0 & 0 & 4 \\
\hline $\mathrm{H} 2$ & DM Risk Gut Fast When Sure & 0 & 0 & 1 & 0 & 0 & 0 & 0 & 0 & 1 & 0 & 1 & 1 & 4 \\
\hline H3 & $\begin{array}{l}\text { DM Risk just when } 100 \% \text { Secure it } \\
\text { will not affect TEC Rules, low Risk }\end{array}$ & 0 & 2 & 0 & 0 & 0 & 0 & 0 & 0 & 0 & 0 & 0 & 0 & 2 \\
\hline $\mathrm{H} 4$ & DM Risk Taking after Measuring & 3 & 1 & 1 & 2 & 1 & 1 & 0 & 0 & 2 & 0 & 1 & 4 & 16 \\
\hline H5 & $\begin{array}{l}\text { DM Risk Taking after Measuring } \\
\text { Example }\end{array}$ & 0 & 0 & 0 & 0 & 0 & 0 & 0 & 0 & 0 & 0 & 0 & 2 & 2 \\
\hline $\mathrm{H} 6$ & $\begin{array}{l}\text { DM Risk Taking when Benefith } \\
\text { worth the Risk }\end{array}$ & 0 & 0 & 0 & 0 & 0 & 0 & 1 & 0 & 0 & 0 & 0 & 1 & 2 \\
\hline $\mathbf{I}$ & DM Risk Team & 2 & $\mathbf{0}$ & $\mathbf{0}$ & $\mathbf{0}$ & 1 & 2 & $\mathbf{0}$ & 2 & 4 & $\mathbf{0}$ & 1 & 2 & 14 \\
\hline 11 & DM Mexicans are slow in DM & 0 & 0 & 0 & 0 & 1 & 0 & 0 & 0 & 0 & 0 & 0 & 0 & 1 \\
\hline 12 & $\begin{array}{l}\text { DM Risk in order to support and } \\
\text { thrust in your Team }\end{array}$ & 0 & 0 & 0 & 0 & 0 & 1 & 0 & 0 & 0 & 0 & 0 & 1 & 2 \\
\hline 13 & DM Risk Team --Y ++N & 0 & 0 & 0 & 0 & 0 & 0 & 0 & 1 & 1 & 0 & 1 & 0 & 3 \\
\hline 14 & $\begin{array}{l}\text { DM Risk Team }++\mathrm{Y} \text { en DDP }++\mathrm{N} \text { en } \\
\text { DDC }\end{array}$ & 0 & 0 & 0 & 0 & 0 & 0 & 0 & 0 & 1 & 0 & 0 & 0 & 1 \\
\hline
\end{tabular}


DM Risk Team everyone Must

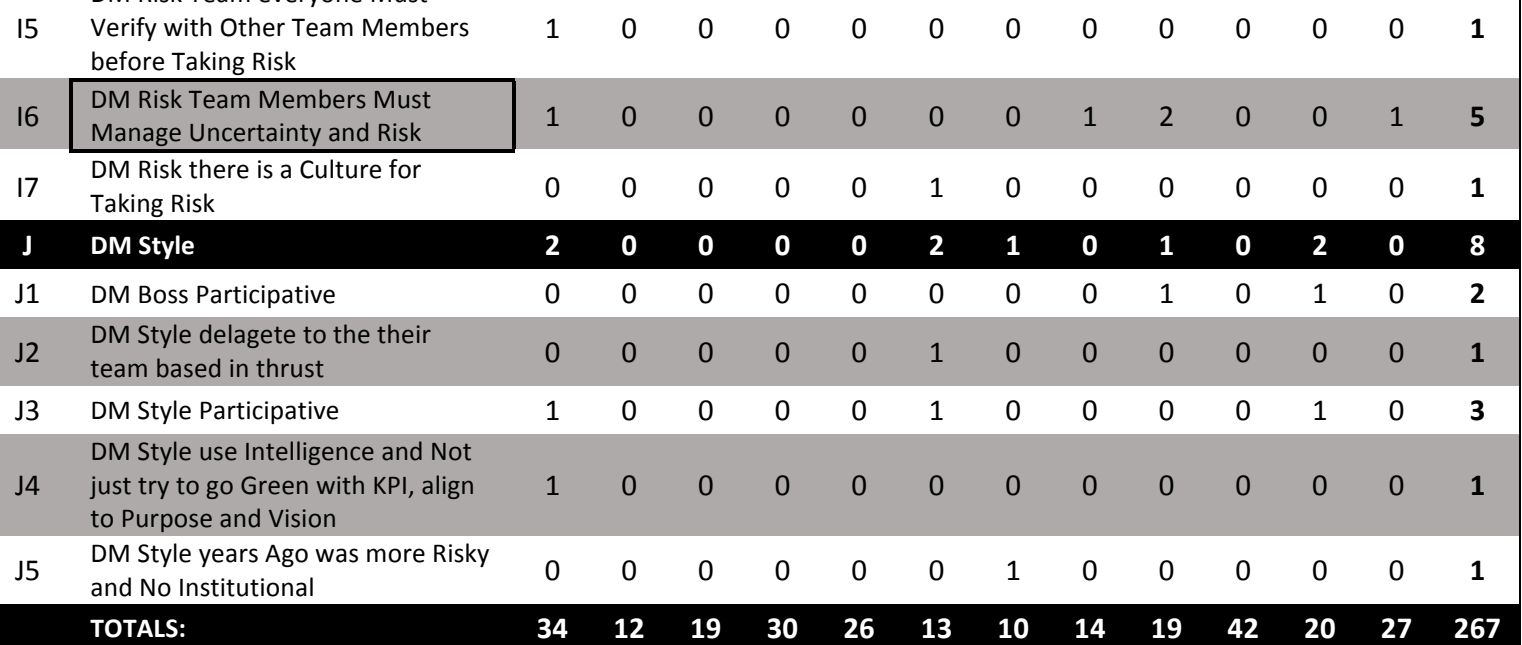

Table 12. Code and Extended Codes for

Risk and Uncertainty in DM Theme at Interviewee detailed.

\begin{tabular}{|c|c|c|}
\hline$\#$ & CODE & MENTIONS \\
\hline A & DM Fast Theme & 29 \\
\hline A1 & Boss DM Like Bosses that are Fast for DM & 2 \\
\hline A2 & DM Being Attached to the Rules Makes you Slower in DM & 4 \\
\hline A3 & DM Employeers want Fast DM workers & 1 \\
\hline A4 & DM Fast taking Decisions & 4 \\
\hline A5 & DM HIGH DIRECTORS in TEC are Fast because the tight Competence & 2 \\
\hline A6 & DM Mexicans are slow in DM & 1 \\
\hline A7 & DM Risk better to apologize but do it & 7 \\
\hline A8 & DM Risk Gut Fast When Sure & 4 \\
\hline A9 & DM Risk GUT NO Like Boss Attached to Regulations $100 \%$ & 2 \\
\hline A10 & DM Risk just when $100 \%$ Secure it will not affect TEC Rules, low Risk & 2 \\
\hline B & DM Risk Boss and High Directors & 47 \\
\hline B1 & Boss DM Like Bosses that are Fast for DM & 2 \\
\hline B2 & Boss DM Risk Support for Mistake & 6 \\
\hline B3 & Boss DM Risk Support for Risk & 8 \\
\hline B4 & DM Risk Boss & 8 \\
\hline B5 & DM Risk Boss Aligned when visions are aligned & 2 \\
\hline B6 & DM Risk Boss Low Risk & 6 \\
\hline B7 & DM Risk Boss not Aligned not Support & 1 \\
\hline B8 & DM Risk Boss Very High & 3 \\
\hline
\end{tabular}




\begin{tabular}{|c|c|c|}
\hline B9 & DM Risk GUT NO Like Boss Attached to Regulations $100 \%$ & 2 \\
\hline B10 & DM Risk Important in HIGH DIRECTORS & 1 \\
\hline B11 & DM Risk Mistake non Support from Boss & 1 \\
\hline B12 & DM Risk Reduce learning from Experience & 2 \\
\hline B13 & Team Successor Selected more Manage Risk (HIGH DIRECTOR) & 5 \\
\hline $\mathbf{C}$ & DM Risk Gut & 48 \\
\hline $\mathrm{C} 1$ & DM Risk Gut based on KPI & 1 \\
\hline $\mathrm{C} 2$ & DM Risk Gut Example & 6 \\
\hline C3 & DM Risk Gut Fast When Sure & 4 \\
\hline C4 & DM Risk Gut He is not Clear Confuse Response & 1 \\
\hline C5 & DM Risk Gut Important in DM & 12 \\
\hline C6 & DM Risk Gut Important in HIGH DIRECTORS & 7 \\
\hline C7 & DM Risk Gut is Because you are not Expert in Everything (confuse answer) & 1 \\
\hline C8 & DM Risk Gut is Pattern (AGREGAR) & 1 \\
\hline $\mathrm{C9}$ & DM Risk Gut Medium & 9 \\
\hline C10 & DM Risk GUT NO Like Boss Attached to Regulations $100 \%$ & 2 \\
\hline C11 & DM Risk Gut use after Validation No DM alone & 4 \\
\hline D & DM Risk High & 32 \\
\hline D1 & DM HIGH DIRECTORS in TEC are Fast because the tight Competence & 2 \\
\hline D2 & DM Risk better to apologize but do it & 7 \\
\hline D3 & DM Risk Boss Very High & 3 \\
\hline D4 & DM Risk High & 7 \\
\hline D5 & DM Risk High Example & 7 \\
\hline D6 & DM Risk take Decision it does not Matter if there is a Price to Pay & 3 \\
\hline D7 & DM Risk take it because Competence is Not Seat and Static & 2 \\
\hline D8 & DM Risk there is a Culture for Taking Risk & 1 \\
\hline $\mathbf{E}$ & DM Risk Low & 6 \\
\hline E1 & DM Risk just when $100 \%$ Secure it will not affect TEC Rules, low Risk & 2 \\
\hline E2 & DM Risk Low & 1 \\
\hline E3 & DM Risk When Sure & 3 \\
\hline $\mathbf{F}$ & DM Risk Medium & 34 \\
\hline $\mathrm{F} 1$ & DM Mexicans are slow in DM & 1 \\
\hline $\mathrm{F} 2$ & DM Risk Medium & 9 \\
\hline F3 & DM Risk Medium Example & 9 \\
\hline F4 & DM Risk Medium to Low & 7 \\
\hline F5 & DM Risk Medium to Low Example & 2 \\
\hline F6 & DM Risk Mexicans are afraid of DM for fear & 1 \\
\hline F7 & DM Risk When No Vital Decisions & 2 \\
\hline F8 & DM Risk When Sure & 3 \\
\hline G & DM Risk Mistake & 19 \\
\hline G1 & Boss DM Risk Support for Mistake & 6 \\
\hline $\mathrm{G} 2$ & DM Risk Mistake Its Ok But Not Repeat Same Error & 7 \\
\hline G3 & DM Risk Mistake No Problem but Never Hide Information (thrust) & 1 \\
\hline
\end{tabular}




\begin{tabular}{|c|c|c|}
\hline G4 & DM Risk Mistake non Support from Boss & 1 \\
\hline G5 & DM Risk Mistake Support when Happens & 4 \\
\hline $\mathbf{H}$ & DM Risk need to chek & 30 \\
\hline $\mathrm{H} 1$ & DM Being Attached to the Rules Makes you Slower in DM & 4 \\
\hline $\mathrm{H} 2$ & DM Risk Gut Fast When Sure & 4 \\
\hline H3 & DM Risk just when $100 \%$ Secure it will not affect TEC Rules, low Risk & 2 \\
\hline H4 & DM Risk Taking after Measuring & 16 \\
\hline H5 & DM Risk Taking after Measuring Example & 2 \\
\hline H6 & DM Risk Taking when Benefith worth the Risk & 2 \\
\hline $\mathbf{I}$ & DM Risk Team & 14 \\
\hline 11 & DM Mexicans are slow in DM & 1 \\
\hline 12 & DM Risk in order to support and thrust in your Team & 2 \\
\hline 13 & DM Risk Team --Y ++N & 3 \\
\hline 14 & DM Risk Team ++Y en DDP ++N en DDC & 1 \\
\hline 15 & DM Risk Team everyone Must Verify with Other Team Members before Taking Risk & 1 \\
\hline 16 & DM Risk Team Members Must Manage Uncertainty and Risk & 5 \\
\hline 17 & DM Risk there is a Culture for Taking Risk & 1 \\
\hline J & DM Style & 8 \\
\hline $\mathrm{J} 1$ & DM Boss Participative & 2 \\
\hline $\mathrm{J} 2$ & DM Style delagete to the their team based in thrust & 1 \\
\hline $\mathrm{J} 3$ & DM Style Participative & 3 \\
\hline J4 & $\begin{array}{l}\text { DM Style use Intelligence and Not just try to go Green with KPI, align to Purpose and } \\
\text { Vision }\end{array}$ & 1 \\
\hline $\mathrm{J} 5$ & DM Style years Ago was more Risky and No Institutional & 1 \\
\hline & TOTALS: & 267 \\
\hline
\end{tabular}

Table 13. Code and Extended Codes for

Risk and Uncertainty in DM Theme.

\begin{tabular}{|c|c|c|c|c|c|c|c|c|c|c|c|c|c|c|c|}
\hline$\#$ & Reduced Code & $\frac{\text { 을 }}{3}$ & $\frac{3}{\text { 公 }}$ & $\stackrel{8}{2}$ & 这 & 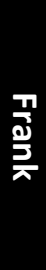 & 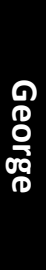 & $\begin{array}{l}\frac{D}{8} \\
\frac{1}{7} \\
\text { D }\end{array}$ & ڤ్ & ட் & $\begin{array}{l}\frac{0}{10} \\
\stackrel{D}{7}\end{array}$ & 题 & $\begin{array}{l}3 \\
\frac{0}{2}\end{array}$ & $\begin{array}{l}\frac{3}{2} \\
\frac{3}{2} \\
\frac{0}{n} \\
\frac{n}{n}\end{array}$ & 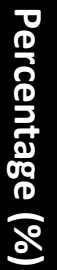 \\
\hline A & Strategy Alignment & 5 & 1 & 5 & 2 & 4 & 1 & 2 & 2 & 1 & 1 & 4 & 1 & 29 & 7 \\
\hline B & Strategy Boss & 2 & 1 & 3 & 1 & 0 & 1 & 0 & 4 & 2 & 0 & 2 & 0 & 16 & 4 \\
\hline C & Strategy Interviewed & 24 & 0 & 25 & 5 & 2 & 10 & 16 & 7 & 15 & 20 & 33 & 15 & 172 & 43 \\
\hline D & $\begin{array}{r}\text { Strategy Interviewed } \\
\text { Differentiation }\end{array}$ & 7 & 0 & 6 & 3 & 0 & 6 & 2 & 0 & 4 & 10 & 8 & 7 & 53 & 13 \\
\hline $\mathrm{E}$ & Strategy Planning & 0 & 0 & 2 & 0 & 2 & 0 & 0 & 0 & 2 & 1 & 0 & 2 & 9 & 2 \\
\hline
\end{tabular}




\begin{tabular}{|c|r|c|c|c|c|c|c|c|c|c|c|c|c|c|c|}
\hline F & Strategy TEC Negative Issues & 0 & 0 & 1 & 3 & 3 & 3 & 1 & 2 & 0 & 1 & 4 & 7 & $\mathbf{2 5}$ & $\mathbf{6}$ \\
\hline G & Strategy Possible Threats & 1 & 0 & 0 & 2 & 2 & 8 & 8 & 0 & 0 & 1 & 3 & 1 & $\mathbf{2 6}$ & $\mathbf{7}$ \\
\hline H & Strategy TEC Time Term & 0 & 0 & 0 & 0 & 1 & 0 & 1 & 1 & 1 & 1 & 1 & 1 & $\mathbf{7}$ & $\mathbf{2}$ \\
\hline I & Strategy TEC & 17 & 0 & 4 & 6 & 2 & 7 & 8 & 1 & 3 & 6 & 5 & 3 & 62 & 16 \\
\hline & TOTALS: & $\mathbf{5 6}$ & $\mathbf{2}$ & $\mathbf{4 6}$ & $\mathbf{2 2}$ & $\mathbf{1 6}$ & $\mathbf{3 6}$ & $\mathbf{3 8}$ & $\mathbf{1 7}$ & $\mathbf{2 8}$ & $\mathbf{4 1}$ & $\mathbf{6 0}$ & $\mathbf{3 7}$ & $\mathbf{3 9 9}$ & $\mathbf{1 0 0}$ \\
\hline
\end{tabular}

Table 14. Codes, Mentions and Percentage of Mentions for Strategy Theme at Interviewee detailed.

\begin{tabular}{|c|c|c|c|}
\hline$\#$ & Reduced Code & MENTIONS & $\begin{array}{c}\text { Percentage } \\
\text { (\%) }\end{array}$ \\
\hline A & Strategy Alignment & 29 & $7 \%$ \\
\hline$B$ & Strategy Boss & 16 & $4 \%$ \\
\hline C & Strategy Interviewed & 172 & $43 \%$ \\
\hline D & $\begin{array}{r}\text { Strategy Interviewed } \\
\text { Differentiation }\end{array}$ & 53 & $13 \%$ \\
\hline$E$ & Strategy Planning & 9 & $2 \%$ \\
\hline $\mathrm{F}$ & Strategy TEC Negative Issues & 25 & $6 \%$ \\
\hline G & Strategy Possible Threats & 26 & $7 \%$ \\
\hline $\mathrm{H}$ & Strategy TEC Time Term & 7 & $2 \%$ \\
\hline 1 & Strategy TEC & 62 & $16 \%$ \\
\hline & TOTALS: & 399 & $100 \%$ \\
\hline
\end{tabular}

Table 15. Codes, Mentions and Percentage of Mentions for Strategy Theme.

\begin{tabular}{|c|c|c|c|c|c|c|c|c|c|c|c|c|c|c|}
\hline$\#$ & CODE & 을 & 裔 & ฏ르 & 峁 & 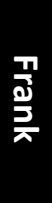 & 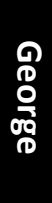 & $\frac{7}{\frac{7}{7}}$ & בָ & ஜே & $\frac{\mathbb{D}}{\stackrel{D}{D}}$ & 邑 & $\frac{3}{2}$ & $\begin{array}{l}\frac{3}{2} \\
\frac{1}{0} \\
\frac{2}{n}\end{array}$ \\
\hline A & Strategy Alignment & 5 & 1 & 5 & 2 & 4 & 1 & 2 & 2 & 1 & 1 & 4 & 1 & 29 \\
\hline A1 & $\begin{array}{r}\text { Strategy Alignment } \\
\text { TEC2020 }\end{array}$ & 4 & 1 & 5 & 2 & 3 & 0 & 1 & 2 & 1 & 1 & 4 & 1 & 25 \\
\hline A2 & $\begin{array}{r}\text { Strategy Interviewed } \\
\text { Alignment through } \\
\text { Convincing People or } \\
\text { Training them }\end{array}$ & 1 & 0 & 0 & 0 & 1 & 0 & 0 & 0 & 0 & 0 & 0 & 0 & 2 \\
\hline A3 & Strategy NOT Alignment & 0 & 0 & 0 & 0 & 0 & 1 & 1 & 0 & 0 & 0 & 0 & 0 & 2 \\
\hline
\end{tabular}




\begin{tabular}{|c|c|c|c|c|c|c|c|c|c|c|c|c|c|c|}
\hline B & Strategy Boss & 2 & 1 & 3 & 1 & 0 & 1 & 0 & 4 & 2 & 0 & 2 & 0 & 16 \\
\hline B1 & Strategy Boss & 1 & 0 & 1 & 0 & 0 & 0 & 0 & 2 & 0 & 0 & 1 & 0 & 5 \\
\hline B2 & Strategy Boss Aligned & 1 & 1 & 2 & 1 & 0 & 1 & 0 & 0 & 2 & 0 & 1 & 0 & 9 \\
\hline B3 & Strategy Boss NOT Aligned & 0 & 0 & 0 & 0 & 0 & 0 & 0 & 2 & 0 & 0 & 0 & 0 & 2 \\
\hline C & Strategy Interviewed & 24 & 0 & 25 & 5 & 2 & 10 & 16 & 7 & 15 & 20 & 33 & 15 & 172 \\
\hline C1 & $\begin{array}{r}\text { Exploitation_NEGATIVE T_ } \\
\text { in Excess make Lack of } \\
\text { Exploration in Strategy }\end{array}$ & 1 & 0 & 0 & 0 & 0 & 0 & 0 & 0 & 0 & 1 & 0 & 1 & 3 \\
\hline C2 & $\begin{array}{r}\text { Exploration How to } \\
\text { Promote He asked the } \\
\text { Team to Down same } \\
\text { Conversations that } \\
\text { Promotes Long Term View }\end{array}$ & 1 & 0 & 0 & 0 & 0 & 0 & 0 & 0 & 0 & 0 & 0 & 0 & 1 \\
\hline C3 & $\begin{array}{r}\text { Strategy Copying Past } \\
\text { Explorative Strategies that } \\
\text { Worked (Path dependence) }\end{array}$ & 0 & 0 & 2 & 0 & 0 & 0 & 0 & 0 & 0 & 0 & 0 & 0 & 2 \\
\hline C4 & $\begin{array}{r}\text { Strategy Interviewed } \\
\text { Academic Quality }\end{array}$ & 3 & 0 & 0 & 0 & 0 & 0 & 0 & 1 & 1 & 3 & 0 & 1 & 9 \\
\hline C5 & $\begin{array}{r}\text { Strategy Interviewed } \\
\text { Academic Quality }\end{array}$ & 1 & 0 & 0 & 0 & 0 & 0 & 0 & 0 & 0 & 1 & 0 & 0 & 2 \\
\hline C6 & $\begin{array}{r}\text { Strategy Interviewed } \\
\text { Adapting through time }\end{array}$ & 1 & 0 & 1 & 0 & 0 & 0 & 0 & 0 & 0 & 0 & 0 & 2 & 4 \\
\hline C7 & $\begin{array}{r}\text { Strategy Interviewed Being } \\
\text { Institutional is Better than } \\
\text { Flexible }\end{array}$ & 0 & 0 & 0 & 0 & 0 & 0 & 1 & 0 & 0 & 0 & 0 & 0 & 1 \\
\hline C8 & $\begin{array}{r}\text { Strategy Interviewed Clima } \\
\text { Organizational }\end{array}$ & 1 & 0 & 0 & 0 & 0 & 0 & 0 & 0 & 0 & 0 & 0 & 0 & 1 \\
\hline C9 & $\begin{array}{r}\text { Strategy Interviewed Clima } \\
\text { Organizational (Leadership } \\
\text { Related) }\end{array}$ & 0 & 0 & 0 & 0 & 0 & 0 & 0 & 1 & 0 & 0 & 0 & 0 & 1 \\
\hline C10 & $\begin{array}{r}\text { Strategy Interviewed } \\
\text { Communication Skills in } \\
\text { Students }\end{array}$ & 0 & 0 & 0 & 0 & 0 & 0 & 0 & 0 & 1 & 0 & 0 & 0 & 1 \\
\hline C11 & $\begin{array}{r}\text { Strategy Interviewed } \\
\text { Competence Copying Short } \\
\text { Term Strategies }\end{array}$ & 0 & 0 & 0 & 0 & 0 & 0 & 0 & 0 & 1 & 0 & 0 & 0 & 1 \\
\hline C12 & $\begin{array}{l}\text { Strategy Interviewed } \\
\text { Complex Factor the } \\
\text { Dispersion of Careers }\end{array}$ & 0 & 0 & 0 & 0 & 0 & 0 & 1 & 0 & 0 & 0 & 0 & 0 & 1 \\
\hline C13 & $\begin{array}{r}\text { Strategy Interviewed } \\
\text { concern about } \\
\text { Diferentiation with Other } \\
\text { Campus }\end{array}$ & 0 & 0 & 0 & 0 & 0 & 0 & 1 & 0 & 0 & 0 & 0 & 0 & 1 \\
\hline C14 & $\begin{array}{r}\text { Strategy Interviewed DDC } \\
\text { should not be in Charge of } \\
\text { Enrollment }\end{array}$ & 0 & 0 & 0 & 0 & 0 & 0 & 1 & 0 & 0 & 0 & 0 & 0 & 1 \\
\hline C15 & $\begin{array}{r}\text { Strategy Interviewed } \\
\text { Development of Directors } \\
\text { (Leadership Related) }\end{array}$ & 1 & 0 & 1 & 1 & 0 & 0 & 0 & 1 & 0 & 0 & 0 & 0 & 4 \\
\hline C16 & $\begin{array}{r}\text { Strategy Interviewed } \\
\text { Ecosistem Students } \\
\text { Experiencie }\end{array}$ & 1 & 0 & 0 & 0 & 0 & 1 & 1 & 0 & 0 & 0 & 1 & 0 & 4 \\
\hline C17 & $\begin{array}{l}\text { Strategy Interviewed } \\
\text { Ecosistems of Career }\end{array}$ & 0 & 0 & 0 & 0 & 0 & 2 & 0 & 0 & 0 & 2 & 0 & 1 & 5 \\
\hline C18 & $\begin{array}{r}\text { Strategy Interviewed } \\
\text { Ecosistems of Career } \\
\text { Winning International } \\
\text { Awards }\end{array}$ & 0 & 0 & 0 & 0 & 0 & 0 & 0 & 0 & 0 & 1 & 0 & 0 & 1 \\
\hline C19 & $\begin{array}{r}\text { Strategy Interviewed } \\
\text { Employability }\end{array}$ & 1 & 0 & 0 & 0 & 0 & 0 & 0 & 0 & 1 & 0 & 0 & 0 & 2 \\
\hline C2O & $\begin{array}{l}\text { Strategy Interviewed } \\
\text { Enrollment focus on } \\
\text { Enrollment of Students, }\end{array}$ & 0 & 0 & 1 & 0 & 0 & 0 & 0 & 0 & 0 & 1 & 0 & 0 & 2 \\
\hline
\end{tabular}




\begin{tabular}{|c|c|c|c|c|c|c|c|c|c|c|c|c|c|c|}
\hline & $\begin{array}{r}\text { Reduction of Scolarships } \\
\text { and Retention in Prepa TEC }\end{array}$ & & & & & & & & & & & & & \\
\hline C21 & $\begin{array}{r}\text { Strategy Interviewed } \\
\text { Enrollment Get Better } \\
\text { Students, selectivity }\end{array}$ & 0 & 0 & 0 & 1 & 0 & 0 & 0 & 0 & 0 & 0 & 0 & 0 & 1 \\
\hline C22 & $\begin{array}{r}\text { Strategy Interviewed } \\
\text { Enrollment of Better } \\
\text { Students through Academic } \\
\text { Quality }\end{array}$ & 0 & 0 & 1 & 1 & 0 & 0 & 0 & 0 & 0 & 1 & 0 & 0 & 3 \\
\hline C23 & $\begin{array}{r}\text { Strategy Interviewed } \\
\text { Facilities }\end{array}$ & 0 & 0 & 0 & 0 & 0 & 0 & 0 & 0 & 0 & 0 & 1 & 0 & 1 \\
\hline C24 & $\begin{array}{r}\text { Strategy Interviewed } \\
\text { Facilities }\end{array}$ & 0 & 0 & 0 & 0 & 0 & 0 & 0 & 0 & 0 & 0 & 1 & 0 & 1 \\
\hline C25 & $\begin{array}{r}\text { Strategy Interviewed } \\
\text { Finantial Health }\end{array}$ & 1 & 0 & 0 & 0 & 0 & 0 & 0 & 0 & 0 & 0 & 0 & 0 & 1 \\
\hline C26 & $\begin{array}{r}\text { Strategy Interviewed } \\
\text { Finantial Health use } \\
\text { Executive Education to } \\
\text { Have Money for Bachelor } \\
\text { Projects Finantial Health }\end{array}$ & 1 & 0 & 0 & 0 & 0 & 0 & 0 & 0 & 0 & 0 & 0 & 0 & 1 \\
\hline C27 & $\begin{array}{r}\text { Strategy Interviewed } \\
\text { Flexibility }\end{array}$ & 1 & 0 & 0 & 0 & 0 & 0 & 0 & 0 & 0 & 0 & 2 & 0 & 3 \\
\hline C28 & $\begin{array}{r}\text { Strategy Interviewed have } \\
\text { big Vision }\end{array}$ & 2 & 0 & 3 & 0 & 0 & 0 & 0 & 0 & 0 & 1 & 0 & 0 & 6 \\
\hline C29 & $\begin{array}{l}\text { Strategy Interviewed } \\
\text { Important Convince Team } \\
\text { about KPI instead Force } \\
\text { them (Leadership Related) }\end{array}$ & 1 & 0 & 0 & 0 & 0 & 0 & 0 & 0 & 0 & 0 & 0 & 0 & 1 \\
\hline C30 & $\begin{array}{r}\text { Strategy Interviewed } \\
\text { Increase the Impact, } \\
\text { Position and Prestige in } \\
\text { Region not just the City, } \\
\text { using and communicating } \\
\text { stregnths of all campuses } \\
\text { and initiatives of TEC } 21 \\
\text { such semana i } \\
\end{array}$ & 0 & 0 & 3 & 0 & 0 & 0 & 0 & 0 & 0 & 0 & 0 & 0 & 3 \\
\hline C31 & $\begin{array}{r}\text { Strategy Interviewed } \\
\text { Innovation Educative + } \\
\text { Vertical Workshop + } \\
\text { iSemester + Iweek }\end{array}$ & 0 & 0 & 0 & 0 & 0 & 0 & 1 & 3 & 3 & 2 & 5 & 1 & 15 \\
\hline C32 & $\begin{array}{l}\text { Strategy Interviewed } \\
\text { Integrity (Leadership) }\end{array}$ & 2 & 0 & 0 & 0 & 0 & 0 & 0 & 0 & 0 & 0 & 0 & 0 & 2 \\
\hline C33 & $\begin{array}{l}\text { Strategy Interviewed } \\
\text { Internationalization }\end{array}$ & 1 & 0 & 1 & 0 & 0 & 0 & 0 & 0 & 0 & 3 & 0 & 0 & 5 \\
\hline C34 & $\begin{array}{r}\text { Strategy Interviewed KPI } \\
\text { Follow and Deployment } \\
\text { Top Down }\end{array}$ & 0 & 0 & 0 & 0 & 1 & 0 & 0 & 0 & 0 & 0 & 0 & 0 & 1 \\
\hline C35 & $\begin{array}{r}\text { Strategy Interviewed } \\
\text { Leadership in Students }\end{array}$ & 0 & 0 & 0 & 0 & 0 & 0 & 0 & 0 & 1 & 0 & 0 & 1 & 2 \\
\hline C36 & $\begin{array}{r}\text { Strategy Interviewed } \\
\text { Negative Attitude }\end{array}$ & 0 & 0 & 0 & 0 & 0 & 2 & 0 & 0 & 0 & 0 & 0 & 0 & 2 \\
\hline C37 & $\begin{array}{r}\text { Strategy Interviewed NOT } \\
\text { own Differentiators as } \\
\text { Division/Campus }\end{array}$ & 0 & 0 & 0 & 0 & 0 & 2 & 0 & 0 & 0 & 0 & 0 & 0 & 2 \\
\hline C38 & $\begin{array}{r}\text { Strategy Interviewed } \\
\text { Prestige }\end{array}$ & 0 & 0 & 1 & 0 & 0 & 0 & 0 & 0 & 0 & 0 & 2 & 2 & 5 \\
\hline C39 & $\begin{array}{r}\text { Strategy Interviewed } \\
\text { Prestige Interviewed is } \\
\text { based in seize the Prestige } \\
\text { of TEC and TEC21 }\end{array}$ & 0 & 0 & 2 & 0 & 0 & 1 & 0 & 0 & 0 & 0 & 0 & 0 & 3 \\
\hline C40 & $\begin{array}{r}\text { Strategy Interviewed } \\
\text { Prestige Interviewed Prepa } \\
\text { TEC size and prestige } \\
\end{array}$ & 0 & 0 & 1 & 0 & 0 & 0 & 0 & 0 & 0 & 0 & 0 & 0 & 1 \\
\hline C41 & $\begin{array}{r}\text { Strategy Interviewed } \\
\text { Professors }\end{array}$ & 0 & 0 & 0 & 0 & 0 & 1 & 0 & 1 & 4 & 1 & 5 & 0 & 12 \\
\hline
\end{tabular}




\begin{tabular}{|c|c|c|c|c|c|c|c|c|c|c|c|c|c|c|}
\hline C42 & $\begin{array}{r}\text { Strategy Interviewed } \\
\text { Professors Cooperation } \\
\text { With Faculty of Other } \\
\text { Campus }\end{array}$ & 0 & 0 & 0 & 0 & 0 & 0 & 0 & 0 & 0 & 0 & 0 & 1 & 1 \\
\hline C43 & $\begin{array}{r}\text { Strategy Interviewed } \\
\text { Professors General }\end{array}$ & 2 & 0 & 0 & 1 & 0 & 0 & 0 & 0 & 0 & 1 & 0 & 1 & 5 \\
\hline C44 & $\begin{array}{r}\text { Strategy Interviewed } \\
\text { Professors International } \\
\text { Presence } \\
\end{array}$ & 0 & 0 & 1 & 0 & 0 & 1 & 0 & 0 & 1 & 2 & 3 & 1 & 9 \\
\hline C45 & $\begin{array}{r}\text { Strategy Interviewed } \\
\text { Professors Vinculados }\end{array}$ & 0 & 0 & 0 & 0 & 0 & 0 & 0 & 0 & 0 & 0 & 2 & 1 & 3 \\
\hline C46 & $\begin{array}{r}\text { Strategy Interviewed } \\
\text { Quality of Service Student } \\
\text { and Family }\end{array}$ & 0 & 0 & 0 & 0 & 0 & 0 & 0 & 0 & 2 & 0 & 0 & 0 & 2 \\
\hline C47 & $\begin{array}{r}\text { Strategy Interviewed say } \\
\text { Processes must be Solid in } \\
\text { order to Advance in } \\
\text { Strategy (Ambi Related) }\end{array}$ & 0 & 0 & 0 & 0 & 0 & 0 & 1 & 0 & 0 & 0 & 0 & 0 & 1 \\
\hline C48 & $\begin{array}{l}\text { Strategy Interviewed Some } \\
\text { KPI Non In Success Factors }\end{array}$ & 0 & 0 & 1 & 0 & 0 & 0 & 1 & 0 & 0 & 0 & 1 & 0 & 3 \\
\hline C49 & $\begin{array}{r}\text { Strategy Interviewed TEC21 } \\
\text { model }\end{array}$ & 0 & 0 & 0 & 1 & 0 & 0 & 0 & 0 & 0 & 0 & 0 & 0 & 1 \\
\hline C50 & $\begin{array}{l}\text { Strategy Interviewed the } \\
\text { Differentiation in a Program } \\
\text { must not depend on the } \\
\text { DDC but the Solid Processes }\end{array}$ & 0 & 0 & 0 & 0 & 0 & 0 & 1 & 0 & 0 & 0 & 0 & 0 & 1 \\
\hline C51 & $\begin{array}{l}\text { Strategy Interviewed the } \\
\text { TEC is NOT a Franquicia } \\
\text { there Should be Exploration }\end{array}$ & 0 & 0 & 1 & 0 & 0 & 0 & 0 & 0 & 0 & 0 & 0 & 0 & 1 \\
\hline C52 & $\begin{array}{r}\text { Strategy Interviewed } \\
\text { Vocacionamiento } \\
\text { Automotriz }\end{array}$ & 0 & 0 & 0 & 0 & 0 & 0 & 0 & 0 & 0 & 0 & 1 & 0 & 1 \\
\hline C53 & $\begin{array}{r}\text { Strategy Interviewed } \\
\text { Vocacionamiento } \\
\text { Enterpreneurship }\end{array}$ & 0 & 0 & 0 & 0 & 0 & 0 & 0 & 0 & 0 & 0 & 3 & 0 & 3 \\
\hline C54 & $\begin{array}{l}\text { Strategy Interviewed } \\
\text { Vocacionamiento Focus to } \\
\text { Reduce Careers to those } \\
\text { that fit to Vocacionamiento }\end{array}$ & 0 & 0 & 0 & 0 & 0 & 0 & 1 & 0 & 0 & 0 & 0 & 0 & 1 \\
\hline C55 & $\begin{array}{r}\text { Strategy Interviewed } \\
\text { Vocacionamiento } \\
\text { Innovation }\end{array}$ & 0 & 0 & 0 & 0 & 0 & 0 & 0 & 0 & 0 & 0 & 2 & 0 & 2 \\
\hline C56 & $\begin{array}{r}\text { Strategy Interviewed } \\
\text { Vocacionamiento } \\
\text { Manufactura }\end{array}$ & 0 & 0 & 0 & 0 & 0 & 0 & 1 & 0 & 0 & 0 & 0 & 0 & 1 \\
\hline C57 & $\begin{array}{r}\text { Strategy Interviewed } \\
\text { Vocacionamiento } \\
\text { Mechatronics }\end{array}$ & 0 & 0 & 0 & 0 & 0 & 0 & 2 & 0 & 0 & 0 & 0 & 0 & 2 \\
\hline C58 & $\begin{array}{r}\text { Strategy Interviewed } \\
\text { Vocacionamiento Not } \\
\text { Depent in DDC the Strategy } \\
\text { of Career but Solidity of } \\
\text { Processes and Seize } \\
\text { Strengths of Region }\end{array}$ & 0 & 0 & 0 & 0 & 0 & 0 & 2 & 0 & 0 & 0 & 0 & 0 & 2 \\
\hline C59 & $\begin{array}{r}\text { Strategy Interviewed } \\
\text { Vocacionamiento Sin } \\
\text { Definir }\end{array}$ & 1 & 0 & 3 & 0 & 0 & 0 & 0 & 0 & 0 & 0 & 0 & 0 & 4 \\
\hline C60 & $\begin{array}{r}\text { Strategy Interviewed when } \\
\text { Arrived to Position was } \\
\text { Essential Listening People } \\
\text { and being Emphatic } \\
\text { (Leadership Related) }\end{array}$ & 0 & 0 & 0 & 0 & 0 & 0 & 1 & 0 & 0 & 0 & 0 & 0 & 1 \\
\hline C61 & $\begin{array}{r}\text { Strategy Interviewed Worst } \\
\text { Scenery Method }\end{array}$ & 0 & 0 & 0 & 0 & 0 & 0 & 0 & 0 & 0 & 0 & 0 & 2 & 2 \\
\hline C62 & $\begin{array}{r}\text { Strategy Strategic Vision in } \\
\text { the Interviewed }\end{array}$ & 1 & 0 & 2 & 0 & 1 & 0 & 0 & 0 & 0 & 0 & 4 & 0 & 8 \\
\hline
\end{tabular}




\begin{tabular}{|c|c|c|c|c|c|c|c|c|c|c|c|c|c|c|}
\hline D & $\begin{array}{r}\text { Strategy Interviewed } \\
\text { Differentiation }\end{array}$ & 7 & 0 & 6 & 3 & 0 & 6 & 2 & 0 & 4 & 10 & 8 & 7 & 53 \\
\hline D1 & $\begin{array}{r}\text { Strategy Interviewed } \\
\text { Academic Quality }\end{array}$ & 1 & 0 & 0 & 0 & 0 & 0 & 0 & 0 & 0 & 1 & 0 & 0 & 2 \\
\hline D2 & $\begin{array}{r}\text { Strategy Interviewed Clima } \\
\text { Organizational }\end{array}$ & 1 & 0 & 0 & 0 & 0 & 0 & 0 & 0 & 0 & 0 & 0 & 0 & 1 \\
\hline D3 & $\begin{array}{r}\text { Strategy Interviewed } \\
\text { Communication Skills in } \\
\text { Students }\end{array}$ & 0 & 0 & 0 & 0 & 0 & 0 & 0 & 0 & 1 & 0 & 0 & 0 & 1 \\
\hline D4 & $\begin{array}{r}\text { Strategy Interviewed } \\
\text { concern about } \\
\text { Diferentiation with Other } \\
\text { Campus }\end{array}$ & 0 & 0 & 0 & 0 & 0 & 0 & 1 & 0 & 0 & 0 & 0 & 0 & 1 \\
\hline D5 & $\begin{array}{l}\text { Strategy Interviewed } \\
\text { Ecosistems of Career }\end{array}$ & 0 & 0 & 0 & 0 & 0 & 2 & 0 & 0 & 0 & 2 & 0 & 1 & 5 \\
\hline D6 & $\begin{array}{r}\text { Strategy Interviewed } \\
\text { Ecosistems of Career } \\
\text { Winning International } \\
\text { Awards }\end{array}$ & 0 & 0 & 0 & 0 & 0 & 0 & 0 & 0 & 0 & 1 & 0 & 0 & 1 \\
\hline D7 & $\begin{array}{r}\text { Strategy Interviewed } \\
\text { Employability }\end{array}$ & 1 & 0 & 0 & 0 & 0 & 0 & 0 & 0 & 1 & 0 & 0 & 0 & 2 \\
\hline D8 & $\begin{array}{l}\text { Strategy Interviewed } \\
\text { Enrollment Get Better } \\
\text { Students, selectivity }\end{array}$ & 0 & 0 & 0 & 1 & 0 & 0 & 0 & 0 & 0 & 0 & 0 & 0 & 1 \\
\hline D9 & $\begin{array}{r}\text { Strategy Interviewed } \\
\text { Facilities }\end{array}$ & 0 & 0 & 0 & 0 & 0 & 0 & 0 & 0 & 0 & 0 & 1 & 0 & 1 \\
\hline D10 & $\begin{array}{r}\text { Strategy Interviewed } \\
\text { Finantial Health } \\
\end{array}$ & 1 & 0 & 0 & 0 & 0 & 0 & 0 & 0 & 0 & 0 & 0 & 0 & 1 \\
\hline D11 & $\begin{array}{r}\text { Strategy Interviewed } \\
\text { Internationalization }\end{array}$ & 1 & 0 & 1 & 0 & 0 & 0 & 0 & 0 & 0 & 3 & 0 & 0 & 5 \\
\hline D12 & $\begin{array}{r}\text { Strategy Interviewed } \\
\text { Leadership in Students }\end{array}$ & 0 & 0 & 0 & 0 & 0 & 0 & 0 & 0 & 1 & 0 & 0 & 1 & 2 \\
\hline D13 & $\begin{array}{r}\text { Strategy Interviewed NOT } \\
\text { own Differentiators as } \\
\text { Division/Campus }\end{array}$ & 0 & 0 & 0 & 0 & 0 & 2 & 0 & 0 & 0 & 0 & 0 & 0 & 2 \\
\hline D14 & $\begin{array}{r}\text { Strategy Interviewed } \\
\text { Prestige }\end{array}$ & 0 & 0 & 1 & 0 & 0 & 0 & 0 & 0 & 0 & 0 & 2 & 2 & 5 \\
\hline D15 & $\begin{array}{r}\text { Strategy Interviewed } \\
\text { Prestige Interviewed is } \\
\text { based in seize the Prestige } \\
\text { of TEC and TEC21 }\end{array}$ & 0 & 0 & 2 & 0 & 0 & 1 & 0 & 0 & 0 & 0 & 0 & 0 & 3 \\
\hline D16 & $\begin{array}{l}\text { Strategy Interviewed } \\
\text { Prestige Interviewed Prepa } \\
\text { TEC size and prestige }\end{array}$ & 0 & 0 & 1 & 0 & 0 & 0 & 0 & 0 & 0 & 0 & 0 & 0 & 1 \\
\hline D17 & $\begin{array}{r}\text { Strategy Interviewed } \\
\text { Professors General }\end{array}$ & 2 & 0 & 0 & 1 & 0 & 0 & 0 & 0 & 0 & 1 & 0 & 1 & 5 \\
\hline D18 & $\begin{array}{r}\text { Strategy Interviewed } \\
\text { Professors International } \\
\text { Presence } \\
\end{array}$ & 0 & 0 & 1 & 0 & 0 & 1 & 0 & 0 & 1 & 2 & 3 & 1 & 9 \\
\hline D19 & $\begin{array}{r}\text { Strategy Interviewed } \\
\text { Professors Vinculados }\end{array}$ & 0 & 0 & 0 & 0 & 0 & 0 & 0 & 0 & 0 & 0 & 2 & 1 & 3 \\
\hline D20 & $\begin{array}{r}\text { Strategy Interviewed TEC21 } \\
\text { model }\end{array}$ & 0 & 0 & 0 & 1 & 0 & 0 & 0 & 0 & 0 & 0 & 0 & 0 & 1 \\
\hline D21 & $\begin{array}{l}\text { Strategy Interviewed the } \\
\text { Differentiation in a Program } \\
\text { must not depend on the } \\
\text { DDC but the Solid Processes }\end{array}$ & 0 & 0 & 0 & 0 & 0 & 0 & 1 & 0 & 0 & 0 & 0 & 0 & 1 \\
\hline $\mathbf{E}$ & Strategy Planning & 0 & 0 & 2 & 0 & 2 & 0 & 0 & 0 & 2 & 1 & 0 & 2 & 9 \\
\hline E1 & Strategic Planning & 0 & 0 & 1 & 0 & 1 & 0 & 0 & 0 & 1 & 1 & 0 & 2 & 6 \\
\hline E2 & $\begin{array}{r}\text { Strategic Planning is } \\
\text { Important }\end{array}$ & 0 & 0 & 0 & 0 & 1 & 0 & 0 & 0 & 0 & 0 & 0 & 0 & 1 \\
\hline E3 & Strategic Planning per Year & 0 & 0 & 1 & 0 & 0 & 0 & 0 & 0 & 1 & 0 & 0 & 0 & 2 \\
\hline $\mathbf{F}$ & $\begin{array}{r}\text { Strategy TEC Negative } \\
\text { Issues }\end{array}$ & 0 & 0 & 1 & 3 & 3 & 3 & 1 & 2 & 0 & 1 & 4 & 7 & 25 \\
\hline
\end{tabular}




\begin{tabular}{|c|c|c|c|c|c|c|c|c|c|c|c|c|c|c|}
\hline F1 & $\begin{array}{r}\text { Strategy TEC Negative } \\
\text { Excess of Servility }\end{array}$ & 0 & 0 & 0 & 0 & 0 & 0 & 0 & 0 & 0 & 0 & 0 & 1 & 1 \\
\hline F2 & $\begin{array}{r}\text { Strategy TEC Negative Hand } \\
\text { Break }\end{array}$ & 0 & 0 & 0 & 0 & 0 & 0 & 0 & 0 & 0 & 0 & 2 & 0 & 2 \\
\hline F3 & $\begin{array}{r}\text { Strategy TEC Negative } \\
\text { Innovation can affect deep } \\
\text { knowledge of students }\end{array}$ & 0 & 0 & 0 & 0 & 1 & 0 & 0 & 0 & 0 & 0 & 0 & 0 & 1 \\
\hline F4 & $\begin{array}{r}\text { Strategy TEC Negative } \\
\text { Innovation that lacks of add } \\
\text { value to students }\end{array}$ & 0 & 0 & 0 & 0 & 1 & 0 & 0 & 0 & 0 & 0 & 0 & 1 & 2 \\
\hline F5 & $\begin{array}{l}\text { Strategy TEC Negative is } \\
\text { Failing in Implementation }\end{array}$ & 0 & 0 & 0 & 1 & 0 & 2 & 0 & 0 & 0 & 0 & 0 & 0 & 3 \\
\hline F6 & $\begin{array}{r}\text { Strategy TEC Negative Lack } \\
\text { of Alignment Top and Down } \\
\text { levels }\end{array}$ & 0 & 0 & 0 & 0 & 0 & 1 & 0 & 0 & 0 & 0 & 0 & 0 & 1 \\
\hline F7 & $\begin{array}{r}\text { Strategy TEC Negative Lack } \\
\text { of Communication Top } \\
\text { Down }\end{array}$ & 0 & 0 & 1 & 1 & 0 & 0 & 0 & 1 & 0 & 0 & 0 & 0 & 3 \\
\hline F8 & $\begin{array}{r}\text { Strategy TEC Negative Lack } \\
\text { of Planning }\end{array}$ & 0 & 0 & 0 & 0 & 0 & 0 & 0 & 0 & 0 & 1 & 0 & 2 & 3 \\
\hline F9 & $\begin{array}{r}\text { Strategy TEC Negative Not } \\
\text { Clarity in KPI for DDC }\end{array}$ & 0 & 0 & 0 & 0 & 0 & 0 & 1 & 0 & 0 & 0 & 0 & 0 & 1 \\
\hline F10 & $\begin{array}{l}\text { Strategy TEC Negative } \\
\text { Processes Centralized }\end{array}$ & 0 & 0 & 0 & 1 & 0 & 0 & 0 & 0 & 0 & 0 & 1 & 0 & 2 \\
\hline F11 & $\begin{array}{r}\text { Strategy TEC Negative } \\
\text { Processes Complex TyC }\end{array}$ & 0 & 0 & 0 & 0 & 0 & 0 & 0 & 1 & 0 & 0 & 1 & 2 & 4 \\
\hline F12 & $\begin{array}{r}\text { Strategy TEC Negative Two } \\
\text { opossite lines educative } \\
\text { innovation and focused in } \\
\text { service }\end{array}$ & 0 & 0 & 0 & 0 & 0 & 0 & 0 & 0 & 0 & 0 & 0 & 1 & 1 \\
\hline F13 & $\begin{array}{r}\text { Strategy TEC Negative } \\
\text { Unconformity and Lack of } \\
\text { Acceptance }\end{array}$ & 0 & 0 & 0 & 0 & 1 & 0 & 0 & 0 & 0 & 0 & 0 & 0 & 1 \\
\hline G & Strategy Possible Threats & 1 & 0 & 0 & 2 & 2 & 8 & 8 & 0 & 0 & 1 & 3 & 1 & 26 \\
\hline G1 & $\begin{array}{r}\text { Strategy Interviewed Threat } \\
\text { a Lot of Activities of DDC }\end{array}$ & 0 & 0 & 0 & 0 & 0 & 0 & 1 & 0 & 0 & 0 & 0 & 0 & 1 \\
\hline G2 & $\begin{array}{r}\text { Strategy Interviewed Threat } \\
\text { Culture of I alreaady did it } \\
\text { and does not Work }\end{array}$ & 0 & 0 & 0 & 0 & 0 & 0 & 1 & 0 & 0 & 0 & 0 & 0 & 1 \\
\hline G3 & $\begin{array}{r}\text { Strategy Interviewed Threat } \\
\text { Inertia against Change and } \\
\text { New Actions }\end{array}$ & 0 & 0 & 0 & 0 & 0 & 0 & 1 & 0 & 0 & 0 & 0 & 0 & 1 \\
\hline G4 & $\begin{array}{l}\text { Strategy Interviewed Threat } \\
\text { Innovation in Educaction }\end{array}$ & 0 & 0 & 0 & 0 & 0 & 0 & 0 & 0 & 0 & 0 & 2 & 0 & 2 \\
\hline G5 & $\begin{array}{r}\text { Strategy Interviewed Threat } \\
\text { International Universities } \\
\text { arriving }\end{array}$ & 0 & 0 & 0 & 0 & 0 & 1 & 1 & 0 & 0 & 0 & 0 & 0 & 2 \\
\hline G6 & $\begin{array}{r}\text { Strategy Interviewed Threat } \\
\text { Mexicans prefer Non } \\
\text { Mexicans Universities }\end{array}$ & 0 & 0 & 0 & 0 & 0 & 1 & 0 & 0 & 0 & 0 & 0 & 0 & 1 \\
\hline G7 & $\begin{array}{r}\text { Strategy Interviewed Threat } \\
\text { New Profile of Students }\end{array}$ & 0 & 0 & 0 & 0 & 0 & 0 & 0 & 0 & 0 & 0 & 1 & 0 & 1 \\
\hline G8 & $\begin{array}{l}\text { Strategy Interviewed Threat } \\
\text { Not Enough Researchers }\end{array}$ & 0 & 0 & 0 & 0 & 0 & 1 & 0 & 0 & 0 & 0 & 0 & 0 & 1 \\
\hline G9 & $\begin{array}{r}\text { Strategy Interviewed Threat } \\
\text { Tec Not Enough Good for } \\
\text { Getting Certain } \\
\text { International Certifications }\end{array}$ & 0 & 0 & 0 & 0 & 0 & 2 & 0 & 0 & 0 & 0 & 0 & 0 & 2 \\
\hline G10 & $\begin{array}{r}\text { Strategy Strategic Vision } \\
\text { Negative the Academic } \\
\text { Hierarchy in Society }\end{array}$ & 0 & 0 & 0 & 0 & 0 & 0 & 1 & 0 & 0 & 0 & 0 & 0 & 1 \\
\hline G11 & $\begin{array}{r}\text { Strategy TEC Negative big } \\
\text { Risk because the Big } \\
\text { Change }\end{array}$ & 1 & 0 & 0 & 1 & 0 & 0 & 0 & 0 & 0 & 0 & 0 & 0 & 2 \\
\hline G12 & $\begin{array}{r}\text { Strategy TEC Negative Not } \\
\text { Clarity in KPI for DDC }\end{array}$ & 0 & 0 & 0 & 0 & 0 & 0 & 1 & 0 & 0 & 0 & 0 & 0 & 1 \\
\hline
\end{tabular}




\begin{tabular}{|c|c|c|c|c|c|c|c|c|c|c|c|c|c|c|}
\hline G13 & $\begin{array}{r}\text { Strategy TEC Negative } \\
\text { Thread Few Full Time } \\
\text { Professors }\end{array}$ & 0 & 0 & 0 & 0 & 1 & 1 & 0 & 0 & 0 & 0 & 0 & 0 & 2 \\
\hline G14 & $\begin{array}{r}\text { Strategy TEC Negative } \\
\text { Threat Different Realities in } \\
\text { Campus }\end{array}$ & 0 & 0 & 0 & 1 & 0 & 0 & 1 & 0 & 0 & 0 & 0 & 0 & 2 \\
\hline G15 & $\begin{array}{r}\text { Strategy TEC Negative } \\
\text { Threat Expensive Model }\end{array}$ & 0 & 0 & 0 & 0 & 1 & 1 & 0 & 0 & 0 & 0 & 0 & 0 & 2 \\
\hline G16 & $\begin{array}{r}\text { Strategy TEC Negative } \\
\text { Threat Focused in KPI can } \\
\text { be Negative to Accomplish } \\
\text { Long Term Vision (R) }\end{array}$ & 0 & 0 & 0 & 0 & 0 & 0 & 1 & 0 & 0 & 0 & 0 & 0 & 1 \\
\hline G17 & $\begin{array}{r}\text { Strategy TEC Negative } \\
\text { Threat Negative Excess of } \\
\text { Drastic and Quick Changes } \\
\text { in Parallel }\end{array}$ & 0 & 0 & 0 & 0 & 0 & 1 & 0 & 0 & 0 & 1 & 0 & 0 & 2 \\
\hline G18 & $\begin{array}{r}\text { Strategy TEC Negative Two } \\
\text { opossite lines educative } \\
\text { innovation and focused in } \\
\text { service }\end{array}$ & 0 & 0 & 0 & 0 & 0 & 0 & 0 & 0 & 0 & 0 & 0 & 1 & 1 \\
\hline $\mathbf{H}$ & Strategy TEC Time Term & 0 & 0 & 0 & 0 & 1 & 0 & 1 & 1 & 1 & 1 & 1 & 1 & 7 \\
\hline H1 & Strategy TEC Term 10 years & 0 & 0 & 0 & 0 & 0 & 0 & 0 & 1 & 0 & 0 & 0 & 0 & 1 \\
\hline $\mathrm{H} 2$ & Strategy TEC Term 3 years & 0 & 0 & 0 & 0 & 0 & 0 & 0 & 0 & 0 & 0 & 0 & 1 & 1 \\
\hline H3 & Strategy TEC Term 5 years & 0 & 0 & 0 & 0 & 0 & 0 & 0 & 0 & 1 & 1 & 1 & 0 & 3 \\
\hline H4 & $\begin{array}{r}\text { Strategy TEC Term much } \\
\text { more than } 10 \text { years }\end{array}$ & 0 & 0 & 0 & 0 & 1 & 0 & 1 & 0 & 0 & 0 & 0 & 0 & 2 \\
\hline I & Strategy TEC & 17 & 0 & 4 & 6 & 2 & 7 & 8 & 1 & 3 & 6 & 5 & 3 & 62 \\
\hline I1 & $\begin{array}{r}\text { Strategy TEC Admires the } \\
\text { Vision of Leaders that } \\
\text { Proposed It when TEC was } \\
\text { Doing Good }\end{array}$ & 4 & 0 & 0 & 0 & 0 & 0 & 0 & 0 & 0 & 0 & 0 & 0 & 4 \\
\hline 12 & $\begin{array}{r}\text { Strategy TEC before Alva No } \\
\text { Alignment No One TEC }\end{array}$ & 0 & 0 & 0 & 1 & 0 & 0 & 2 & 0 & 0 & 0 & 0 & 0 & 3 \\
\hline 13 & $\begin{array}{r}\text { Strategy TEC Campus } \\
\text { Director is more } \\
\text { Exploitation }(T) \text { than } \\
\text { Exploration (R.) }\end{array}$ & 1 & 0 & 0 & 0 & 0 & 0 & 0 & 0 & 0 & 0 & 0 & 0 & 1 \\
\hline 14 & $\begin{array}{r}\text { Strategy TEC Clear } \\
\text { Formulation } \\
\end{array}$ & 0 & 0 & 1 & 1 & 0 & 0 & 1 & 0 & 0 & 0 & 1 & 0 & 4 \\
\hline 15 & $\begin{array}{r}\text { Strategy TEC focus on a } \\
\text { Continius Change because } \\
\text { an Ever Change } \\
\text { Environment }\end{array}$ & 2 & 0 & 0 & 0 & 0 & 0 & 0 & 0 & 0 & 0 & 0 & 0 & 2 \\
\hline 16 & $\begin{array}{r}\text { Strategy TEC focus on } \\
\text { Accomplish Mision }\end{array}$ & 0 & 0 & 1 & 0 & 0 & 0 & 0 & 0 & 0 & 0 & 0 & 0 & 1 \\
\hline 17 & $\begin{array}{r}\text { Strategy TEC focus on } \\
\text { Improve Image to the } \\
\text { Society }\end{array}$ & 0 & 0 & 0 & 0 & 0 & 0 & 0 & 0 & 0 & 2 & 0 & 0 & 2 \\
\hline 18 & $\begin{array}{r}\text { Strategy TEC focus on } \\
\text { Improving Academic } \\
\text { Quality through Good } \\
\text { Professors and Good } \\
\text { Students }\end{array}$ & 0 & 0 & 0 & 0 & 0 & 0 & 0 & 0 & 0 & 2 & 0 & 0 & 2 \\
\hline 19 & $\begin{array}{r}\text { Strategy TEC focus on } \\
\text { Improving in Rankings to be } \\
1 \text { in Mexico }\end{array}$ & 0 & 0 & 0 & 0 & 0 & 0 & 0 & 0 & 0 & 1 & 0 & 0 & 1 \\
\hline I10 & $\begin{array}{r}\text { Strategy TEC focus on KPI } \\
\text { Employability }\end{array}$ & 0 & 0 & 0 & 0 & 0 & 0 & 0 & 0 & 1 & 0 & 0 & 0 & 1 \\
\hline I11 & $\begin{array}{r}\text { Strategy TEC focus on KPI } \\
\text { Internationalization }\end{array}$ & 0 & 0 & 0 & 0 & 0 & 0 & 0 & 0 & 1 & 0 & 0 & 0 & 1 \\
\hline I12 & $\begin{array}{r}\text { Strategy TEC focus on New } \\
\text { Educative Model } \\
\end{array}$ & 2 & 0 & 0 & 0 & 1 & 1 & 0 & 0 & 0 & 0 & 0 & 1 & 5 \\
\hline I13 & $\begin{array}{r}\text { Strategy TEC focus on } \\
\text { Standarize Process and One }\end{array}$ & 0 & 0 & 0 & 1 & 0 & 0 & 1 & 0 & 0 & 0 & 0 & 0 & 2 \\
\hline
\end{tabular}


TEC

\begin{tabular}{|c|c|c|c|c|c|c|c|c|c|c|c|c|c|c|}
\hline I14 & $\begin{array}{r}\text { Strategy TEC focus on } \\
\text { Technology in Education }\end{array}$ & 0 & 0 & 0 & 0 & 0 & 1 & 0 & 0 & 0 & 0 & 0 & 0 & 1 \\
\hline I15 & $\begin{array}{l}\text { Strategy TEC focus on the } \\
\text { Beginning of a Last for Ever } \\
\text { Change Strategy for TEC }\end{array}$ & 1 & 0 & 0 & 0 & 0 & 0 & 0 & 0 & 0 & 0 & 0 & 0 & 1 \\
\hline I16 & $\begin{array}{r}\text { Strategy TEC Good External } \\
\text { Acceptance (Society) }\end{array}$ & 0 & 0 & 0 & 0 & 0 & 0 & 0 & 0 & 0 & 0 & 1 & 0 & 1 \\
\hline 117 & $\begin{array}{r}\text { Strategy TEC in one Phrase } \\
\text { Changes live thorugh } \\
\text { education }\end{array}$ & 0 & 0 & 0 & 1 & 0 & 0 & 0 & 0 & 0 & 0 & 0 & 0 & 1 \\
\hline I18 & $\begin{array}{r}\text { Strategy TEC is a Disruptive } \\
\text { Change }\end{array}$ & 1 & 0 & 0 & 0 & 0 & 0 & 0 & 0 & 0 & 0 & 0 & 0 & 1 \\
\hline I19 & $\begin{array}{l}\text { Strategy TEC it Changed the } \\
\text { Vision of the Interviewed }\end{array}$ & 1 & 0 & 0 & 0 & 0 & 0 & 0 & 0 & 0 & 0 & 0 & 0 & 1 \\
\hline 120 & $\begin{array}{r}\text { Strategy TEC neccesary } \\
\text { because Changing World }\end{array}$ & 2 & 0 & 0 & 0 & 0 & 1 & 0 & 0 & 0 & 0 & 0 & 0 & 3 \\
\hline 121 & $\begin{array}{r}\text { Strategy TEC neccesary } \\
\text { because New Students } \\
\text { Profile }\end{array}$ & 1 & 0 & 0 & 0 & 0 & 0 & 0 & 0 & 0 & 0 & 0 & 0 & 1 \\
\hline 122 & $\begin{array}{r}\text { Strategy TEC neccesary } \\
\text { because several different } \\
\text { Campus }\end{array}$ & 0 & 0 & 0 & 1 & 0 & 0 & 1 & 0 & 0 & 0 & 0 & 0 & 2 \\
\hline 123 & $\begin{array}{r}\text { Strategy TEC Perceived as a } \\
\text { New Educative Model }\end{array}$ & 0 & 0 & 0 & 0 & 0 & 0 & 0 & 1 & 0 & 0 & 0 & 0 & 1 \\
\hline 124 & $\begin{array}{r}\text { Strategy TEC Positive } \\
\text { Attitude }\end{array}$ & 1 & 0 & 1 & 0 & 1 & 2 & 0 & 0 & 0 & 0 & 1 & 0 & 6 \\
\hline 125 & $\begin{array}{r}\text { Strategy TEC Positive } \\
\text { Change }\end{array}$ & 1 & 0 & 1 & 0 & 0 & 2 & 1 & 0 & 0 & 0 & 1 & 0 & 6 \\
\hline 126 & $\begin{array}{r}\text { Strategy TEC Simple } \\
\text { Formulation }\end{array}$ & 0 & 0 & 0 & 0 & 0 & 0 & 0 & 0 & 0 & 0 & 1 & 0 & 1 \\
\hline 127 & $\begin{array}{r}\text { Strategy TEC Strategies are } \\
\text { not generic }\end{array}$ & 0 & 0 & 0 & 1 & 0 & 0 & 2 & 0 & 0 & 0 & 0 & 0 & 3 \\
\hline 128 & $\begin{array}{l}\text { Strategy TEC Students being } \\
\text { Competitive Worldwide }\end{array}$ & 0 & 0 & 0 & 0 & 0 & 0 & 0 & 0 & 1 & 1 & 0 & 2 & 4 \\
\hline & TOTALS: & 56 & 2 & 46 & 22 & 16 & 36 & 38 & 17 & 28 & 41 & 60 & 37 & 399 \\
\hline
\end{tabular}

Table 16. Code and Extended Codes for

Strategy Theme at Interviewee detailed.

\begin{tabular}{|c|l|c|}
\hline$\#$ & \multicolumn{1}{c}{ CODE } & MENTIONS \\
\hline A & Strategy Alignment & $\mathbf{2 9}$ \\
\hline A1 & Strategy Alignment TEC2020 & $\mathbf{2 5}$ \\
\hline A2 & Strategy Interviewed Alignment through Convincing People or Training them & $\mathbf{2}$ \\
\hline A3 & Strategy NOT Alignment & $\mathbf{2}$ \\
\hline B & Strategy Boss & $\mathbf{1 6}$ \\
\hline B1 & Strategy Boss & $\mathbf{5}$ \\
\cline { 2 - 3 } B2 & Strategy Boss Aligned & $\mathbf{2}$ \\
\hline B3 & Strategy Boss NOT Aligned & $\mathbf{1 7 2}$ \\
\hline C & Strategy Interviewed & $\mathbf{3}$ \\
\hline C1 & Exploitation_NEGATIVE T_in Excess make Lack of Exploration in Strategy & $\mathbf{1}$ \\
\hline C2 & Exploration How to Promote He asked the Team to Down same Conversations that & \\
\hline
\end{tabular}




\begin{tabular}{|c|c|c|}
\hline C3 & Strategy Copying Past Explorative Strategies that Worked (Path dependence) & 2 \\
\hline C4 & Strategy Interviewed Academic Quality & 9 \\
\hline C5 & Strategy Interviewed Academic Quality & 2 \\
\hline C6 & Strategy Interviewed Adapting through time & 4 \\
\hline C7 & Strategy Interviewed Being Institutional is Better than Flexible & 1 \\
\hline C8 & Strategy Interviewed Clima Organizational & 1 \\
\hline C9 & Strategy Interviewed Clima Organizational (Leadership Related) & 1 \\
\hline C10 & Strategy Interviewed Communication Skills in Students & 1 \\
\hline C11 & Strategy Interviewed Competence Copying Short Term Strategies & 1 \\
\hline C12 & Strategy Interviewed Complex Factor the Dispersion of Careers & 1 \\
\hline C13 & Strategy Interviewed concern about Diferentiation with Other Campus & 1 \\
\hline C14 & Strategy Interviewed DDC should not be in Charge of Enrollment & 1 \\
\hline C15 & Strategy Interviewed Development of Directors (Leadership Related) & 4 \\
\hline C16 & Strategy Interviewed Ecosistem Students Experiencie & 4 \\
\hline C17 & Strategy Interviewed Ecosistems of Career & 5 \\
\hline C18 & Strategy Interviewed Ecosistems of Career Winning International Awards & 1 \\
\hline C19 & Strategy Interviewed Employability & 2 \\
\hline C20 & $\begin{array}{l}\text { Strategy Interviewed Enrollment focus on Enrollment of Students, Reduction of } \\
\text { Scolarships and Retention in Prepa TEC }\end{array}$ & 2 \\
\hline C21 & Strategy Interviewed Enrollment Get Better Students, selectivity & 1 \\
\hline C22 & Strategy Interviewed Enrollment of Better Students through Academic Quality & 3 \\
\hline C23 & Strategy Interviewed Facilities & 1 \\
\hline C24 & Strategy Interviewed Facilities & 1 \\
\hline C25 & Strategy Interviewed Finantial Health & 1 \\
\hline C26 & $\begin{array}{l}\text { Strategy Interviewed Finantial Health use Executive Education to Have Money for } \\
\text { Bachelor Projects Finantial Health }\end{array}$ & 1 \\
\hline C27 & Strategy Interviewed Flexibility & 3 \\
\hline C28 & Strategy Interviewed have big Vision & 6 \\
\hline C29 & $\begin{array}{l}\text { Strategy Interviewed Important Convince Team about KPI instead Force them } \\
\text { (Leadership Related) }\end{array}$ & 1 \\
\hline C30 & $\begin{array}{l}\text { Strategy Interviewed Increase the Impact, Position and Prestige in Region not just } \\
\text { the City, using and communicating stregnths of all campuses and initiatives of TEC } \\
21 \text { such semana i }\end{array}$ & 3 \\
\hline C31 & Strategy Interviewed Innovation Educative + Vertical Workshop + iSemester + Iweek & 15 \\
\hline C32 & Strategy Interviewed Integrity (Leadership) & 2 \\
\hline C33 & Strategy Interviewed Internationalization & 5 \\
\hline C34 & Strategy Interviewed KPI Follow and Deployment Top Down & 1 \\
\hline C35 & Strategy Interviewed Leadership in Students & 2 \\
\hline C36 & Strategy Interviewed Negative Attitude & 2 \\
\hline C37 & Strategy Interviewed NOT own Differentiators as Division/Campus & 2 \\
\hline C38 & Strategy Interviewed Prestige & 5 \\
\hline C39 & $\begin{array}{l}\text { Strategy Interviewed Prestige Interviewed is based in seize the Prestige of TEC and } \\
\text { TEC21 }\end{array}$ & 3 \\
\hline C40 & Strategy Interviewed Prestige Interviewed Prepa TEC size and prestige & 1 \\
\hline
\end{tabular}




\begin{tabular}{|c|c|c|}
\hline C41 & Strategy Interviewed Professors & 12 \\
\hline C42 & Strategy Interviewed Professors Cooperation With Faculty of Other Campus & 1 \\
\hline C43 & Strategy Interviewed Professors General & 5 \\
\hline C44 & Strategy Interviewed Professors International Presence & 9 \\
\hline $\mathbf{C 4 5}$ & Strategy Interviewed Professors Vinculados & 3 \\
\hline C46 & Strategy Interviewed Quality of Service Student and Family & 2 \\
\hline C47 & $\begin{array}{l}\text { Strategy Interviewed say Processes must be Solid in order to Advance in Strategy } \\
\text { (Ambi Related) }\end{array}$ & 1 \\
\hline C48 & Strategy Interviewed Some KPI Non In Success Factors & 3 \\
\hline C49 & Strategy Interviewed TEC21 model & 1 \\
\hline C50 & $\begin{array}{l}\text { Strategy Interviewed the Differentiation in a Program must not depend on the DDC } \\
\text { but the Solid Processes }\end{array}$ & 1 \\
\hline C51 & Strategy Interviewed the TEC is NOT a Franquicia there Should be Exploration & 1 \\
\hline C52 & Strategy Interviewed Vocacionamiento Automotriz & 1 \\
\hline C53 & Strategy Interviewed Vocacionamiento Enterpreneurship & 3 \\
\hline C54 & $\begin{array}{l}\text { Strategy Interviewed Vocacionamiento Focus to Reduce Careers to those that fit to } \\
\text { Vocacionamiento }\end{array}$ & 1 \\
\hline C55 & Strategy Interviewed Vocacionamiento Innovation & 2 \\
\hline C56 & Strategy Interviewed Vocacionamiento Manufactura & 1 \\
\hline C57 & Strategy Interviewed Vocacionamiento Mechatronics & 2 \\
\hline C58 & $\begin{array}{l}\text { Strategy Interviewed Vocacionamiento Not Depent in DDC the Strategy of Career } \\
\text { but Solidity of Processes and Seize Strengths of Region }\end{array}$ & 2 \\
\hline C59 & Strategy Interviewed Vocacionamiento Sin Definir & 4 \\
\hline C60 & $\begin{array}{l}\text { Strategy Interviewed when Arrived to Position was Essential Listening People and } \\
\text { being Emphatic (Leadership Related) }\end{array}$ & 1 \\
\hline C61 & Strategy Interviewed Worst Scenery Method & 2 \\
\hline C62 & Strategy Strategic Vision in the Interviewed & 8 \\
\hline D & Strategy Interviewed Differentiation & 53 \\
\hline D1 & Strategy Interviewed Academic Quality & 2 \\
\hline D2 & Strategy Interviewed Clima Organizational & 1 \\
\hline D3 & Strategy Interviewed Communication Skills in Students & 1 \\
\hline D4 & Strategy Interviewed concern about Diferentiation with Other Campus & 1 \\
\hline D5 & Strategy Interviewed Ecosistems of Career & 5 \\
\hline D6 & Strategy Interviewed Ecosistems of Career Winning International Awards & 1 \\
\hline D7 & Strategy Interviewed Employability & 2 \\
\hline D8 & Strategy Interviewed Enrollment Get Better Students, selectivity & 1 \\
\hline D9 & Strategy Interviewed Facilities & 1 \\
\hline D10 & Strategy Interviewed Finantial Health & 1 \\
\hline D11 & Strategy Interviewed Internationalization & 5 \\
\hline D12 & Strategy Interviewed Leadership in Students & 2 \\
\hline D13 & Strategy Interviewed NOT own Differentiators as Division/Campus & 2 \\
\hline D14 & Strategy Interviewed Prestige & 5 \\
\hline D15 & $\begin{array}{l}\text { Strategy Interviewed Prestige Interviewed is based in seize the Prestige of TEC and } \\
\text { TEC21 }\end{array}$ & 3 \\
\hline
\end{tabular}




\begin{tabular}{|c|c|c|}
\hline D16 & Strategy Interviewed Prestige Interviewed Prepa TEC size and prestige & 1 \\
\hline D17 & Strategy Interviewed Professors General & 5 \\
\hline D18 & Strategy Interviewed Professors International Presence & 9 \\
\hline D19 & Strategy Interviewed Professors Vinculados & 3 \\
\hline D20 & Strategy Interviewed TEC21 model & 1 \\
\hline D21 & $\begin{array}{l}\text { Strategy Interviewed the Differentiation in a Program must not depend on the DDC } \\
\text { but the Solid Processes }\end{array}$ & 1 \\
\hline $\mathbf{E}$ & Strategy Planning & 9 \\
\hline E1 & Strategic Planning & 6 \\
\hline E2 & Strategic Planning is Important & 1 \\
\hline E3 & Strategic Planning per Year & 2 \\
\hline $\mathbf{F}$ & Strategy TEC Negative Issues & 25 \\
\hline F1 & Strategy TEC Negative Excess of Servility & 1 \\
\hline F2 & Strategy TEC Negative Hand Break & 2 \\
\hline F3 & Strategy TEC Negative Innovation can affect deep knowledge of students & 1 \\
\hline F4 & Strategy TEC Negative Innovation that lacks of add value to students & 2 \\
\hline F5 & Strategy TEC Negative is Failing in Implementation & 3 \\
\hline F6 & Strategy TEC Negative Lack of Alignment Top and Down levels & 1 \\
\hline F7 & Strategy TEC Negative Lack of Communication Top Down & 3 \\
\hline F8 & Strategy TEC Negative Lack of Planning & 3 \\
\hline F9 & Strategy TEC Negative Not Clarity in KPI for DDC & 1 \\
\hline F10 & Strategy TEC Negative Processes Centralized & 2 \\
\hline F11 & Strategy TEC Negative Processes Complex TyC & 4 \\
\hline F12 & $\begin{array}{l}\text { Strategy TEC Negative Two opossite lines educative innovation and focused in } \\
\text { service }\end{array}$ & 1 \\
\hline F13 & Strategy TEC Negative Unconformity and Lack of Acceptance & 1 \\
\hline G & Strategy Possible Threats & 26 \\
\hline G1 & Strategy Interviewed Threat a Lot of Activities of DDC & 1 \\
\hline G2 & Strategy Interviewed Threat Culture of I alreaady did it and does not Work & 1 \\
\hline G3 & Strategy Interviewed Threat Inertia against Change and New Actions & 1 \\
\hline G4 & Strategy Interviewed Threat Innovation in Educaction & 2 \\
\hline G5 & Strategy Interviewed Threat International Universities arriving & 2 \\
\hline G6 & Strategy Interviewed Threat Mexicans prefer Non Mexicans Universities & 1 \\
\hline G7 & Strategy Interviewed Threat New Profile of Students & 1 \\
\hline G8 & Strategy Interviewed Threat Not Enough Researchers & 1 \\
\hline G9 & $\begin{array}{l}\text { Strategy Interviewed Threat Tec Not Enough Good for Getting Certain International } \\
\text { Certifications }\end{array}$ & 2 \\
\hline G10 & Strategy Strategic Vision Negative the Academic Hierarchy in Society & 1 \\
\hline G11 & Strategy TEC Negative big Risk because the Big Change & 2 \\
\hline G12 & Strategy TEC Negative Not Clarity in KPI for DDC & 1 \\
\hline G13 & Strategy TEC Negative Thread Few Full Time Professors & 2 \\
\hline G14 & Strategy TEC Negative Threat Different Realities in Campus & 2 \\
\hline G15 & Strategy TEC Negative Threat Expensive Model & 2 \\
\hline G16 & $\begin{array}{l}\text { Strategy TEC Negative Threat Focused in KPI can be Negative to Accomplish Long } \\
\text { Term Vision (R) }\end{array}$ & 1 \\
\hline
\end{tabular}




\begin{tabular}{|c|c|c|}
\hline G17 & $\begin{array}{l}\text { Strategy TEC Negative Threat Negative Excess of Drastic and Quick Changes in } \\
\text { Parallel }\end{array}$ & 2 \\
\hline G18 & $\begin{array}{l}\text { Strategy TEC Negative Two opossite lines educative innovation and focused in } \\
\text { service }\end{array}$ & 1 \\
\hline $\mathbf{H}$ & Strategy TEC Time Term & 7 \\
\hline H1 & Strategy TEC Term 10 years & 1 \\
\hline H2 & Strategy TEC Term 3 years & 1 \\
\hline H3 & Strategy TEC Term 5 years & 3 \\
\hline H4 & Strategy TEC Term much more than 10 years & 2 \\
\hline $\mathbf{I}$ & Strategy TEC & 62 \\
\hline I1 & $\begin{array}{l}\text { Strategy TEC Admires the Vision of Leaders that Proposed It when TEC was Doing } \\
\text { Good }\end{array}$ & 4 \\
\hline 12 & Strategy TEC before Alva No Alignment No One TEC & 3 \\
\hline 13 & Strategy TEC Campus Director is more Exploitation (T) than Exploration (R.) & 1 \\
\hline 14 & Strategy TEC Clear Formulation & 4 \\
\hline 15 & Strategy TEC focus on a Continius Change because an Ever Change Environment & 2 \\
\hline 16 & Strategy TEC focus on Accomplish Mision & 1 \\
\hline 17 & Strategy TEC focus on Improve Image to the Society & 2 \\
\hline 18 & $\begin{array}{l}\text { Strategy TEC focus on Improving Academic Quality through Good Professors and } \\
\text { Good Students }\end{array}$ & 2 \\
\hline 19 & Strategy TEC focus on Improving in Rankings to be 1 in Mexico & 1 \\
\hline 110 & Strategy TEC focus on KPI Employability & 1 \\
\hline $\mathbf{I 1 1}$ & Strategy TEC focus on KPI Internationalization & 1 \\
\hline 112 & Strategy TEC focus on New Educative Model & 5 \\
\hline 113 & Strategy TEC focus on Standarize Process and One TEC & 2 \\
\hline 114 & Strategy TEC focus on Technology in Education & 1 \\
\hline 115 & Strategy TEC focus on the Beginning of a Last for Ever Change Strategy for TEC & 1 \\
\hline I16 & Strategy TEC Good External Acceptance (Society) & 1 \\
\hline I17 & Strategy TEC in one Phrase Changes live thorugh education & 1 \\
\hline 118 & Strategy TEC is a Disruptive Change & 1 \\
\hline I19 & Strategy TEC it Changed the Vision of the Interviewed & 1 \\
\hline 120 & Strategy TEC neccesary because Changing World & 3 \\
\hline 121 & Strategy TEC neccesary because New Students Profile & 1 \\
\hline 122 & Strategy TEC neccesary because several different Campus & 2 \\
\hline 123 & Strategy TEC Perceived as a New Educative Model & 1 \\
\hline 124 & Strategy TEC Positive Attitude & 6 \\
\hline 125 & Strategy TEC Positive Change & 6 \\
\hline 126 & Strategy TEC Simple Formulation & 1 \\
\hline 127 & Strategy TEC Strategies are not generic & 3 \\
\hline 128 & Strategy TEC Students being Competitive Worldwide & 4 \\
\hline & TOTALS: & 399 \\
\hline
\end{tabular}

Table 17. Code and Extended Codes for Strategy Theme. 


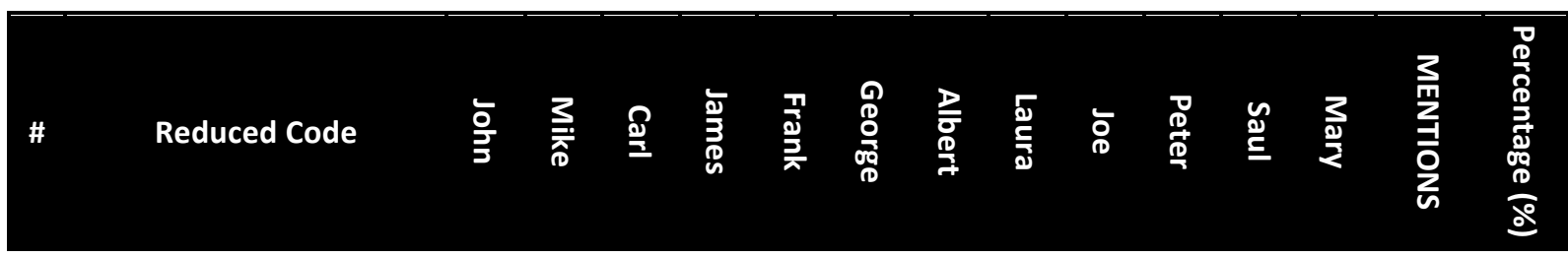

\begin{tabular}{|c|c|c|c|c|c|c|c|c|c|c|c|c|c|c|c|}
\hline $\mathbf{A}$ & Ambidexterity Interviewed & 3 & 2 & 7 & 1 & 1 & 1 & 5 & 1 & 2 & 3 & 7 & 1 & 34 & 12 \\
\hline B & Ambidexterity Successor & 1 & 0 & 1 & 4 & 0 & 2 & 3 & 0 & 0 & 0 & 0 & 1 & 12 & 4 \\
\hline C & $\begin{array}{r}\text { Ambidexterity High } \\
\text { Directors }\end{array}$ & 5 & 0 & 7 & 5 & 1 & 3 & 2 & 1 & 6 & 2 & 1 & 1 & 34 & 12 \\
\hline D & Ambidexterity Team & 2 & 0 & 0 & 1 & 2 & 1 & 2 & 3 & 5 & 2 & 2 & 2 & 22 & 8 \\
\hline $\mathbf{E}$ & $\begin{array}{r}\text { Ambidexterity How } \\
\text { Happens }\end{array}$ & 5 & 2 & 7 & 6 & 1 & 5 & 7 & 7 & 3 & 11 & 6 & 6 & 66 & 23 \\
\hline $\mathbf{F}$ & Ambidexterity Structural & 1 & 0 & 0 & 1 & 0 & 0 & 3 & 0 & 0 & 1 & 1 & 0 & 6 & 2 \\
\hline $\mathbf{G}$ & Ambidexterity Contextual & 3 & 4 & 3 & 2 & 1 & 2 & 9 & 0 & 0 & 4 & 3 & 3 & 34 & 12 \\
\hline H & Ambidexterity Threats & 1 & 1 & 2 & 3 & 0 & 0 & 3 & 4 & 0 & 6 & 0 & 2 & 22 & 8 \\
\hline I & Ambidexterity General & 7 & 0 & 4 & 3 & 3 & 4 & 1 & 2 & 2 & 9 & 3 & 4 & 42 & 15 \\
\hline J & Ambidexterity Boss & 0 & 0 & 1 & 1 & 0 & 0 & 1 & 0 & 4 & 1 & 0 & 1 & 9 & 3 \\
\hline & TOTAL & 28 & 9 & 32 & 27 & 9 & 18 & 36 & 18 & 22 & 39 & 23 & 21 & 281 & 100 \\
\hline
\end{tabular}

Table 18. Codes, Mentions and Percentage of Mentions for Ambidexterity Theme at Interviewee detailed.

\begin{tabular}{|c|c|c|c|}
\hline \# & Code & MENTONS & PERCENTAGE \\
\hline A & Ambidexterity Interviewed & 34 & $12 \%$ \\
\hline B & Ambidexterity Successor & 12 & $4 \%$ \\
\hline C & Ambidexterity High Directors & 34 & $12 \%$ \\
\hline D & Ambidexterity Team & 22 & $8 \%$ \\
\hline $\mathbf{E}$ & Ambidexterity How Happens & 66 & $23 \%$ \\
\hline $\mathbf{F}$ & Ambidexterity Structural & 6 & $2 \%$ \\
\hline G & Ambidexterity Contextual & 34 & $12 \%$ \\
\hline H & Ambidexterity Threats & 22 & $8 \%$ \\
\hline $\mathbf{I}$ & Ambidexterity General & 42 & $15 \%$ \\
\hline J & Ambidexterity Boss & 9 & $3 \%$ \\
\hline & TOTAL & 281 & $100 \%$ \\
\hline
\end{tabular}

Table 19. Codes, Mentions and Percentage of Mentions for Ambidexterity Theme. 


\begin{tabular}{|c|c|c|c|c|c|c|c|c|c|c|c|c|c|c|}
\hline A & Ambidexterity Interviewed & 3 & 2 & 7 & 1 & 1 & 1 & 5 & 1 & 2 & 3 & 7 & 1 & 34 \\
\hline A1 & $\begin{array}{r}\text { Ambidexterity I wish I could } \\
\text { Spend More Time in Exploration } \\
\text { (R) }\end{array}$ & 1 & 0 & 2 & 0 & 0 & 0 & 0 & 0 & 0 & 1 & 0 & 0 & 4 \\
\hline A2 & $\begin{array}{r}\text { Ambidexterity Interviewed }++5 R- \\
-2 T\end{array}$ & 0 & 0 & 0 & 0 & 0 & 0 & 0 & 0 & 0 & 1 & 1 & 0 & 2 \\
\hline A3 & $\begin{array}{r}\text { Ambidexterity Interviewed } \\
\text { Dynamic Depending Needs, bot } \\
\text { more }(T) \text { than }(R)\end{array}$ & 0 & 2 & 0 & 0 & 0 & 0 & 0 & 0 & 0 & 0 & 0 & 0 & 2 \\
\hline A4 & $\begin{array}{r}\text { Ambidexterity Interviewed } \\
\text { Exploration More Mid and Long } \\
\text { Term Person (R) }\end{array}$ & 0 & 0 & 3 & 0 & 1 & 1 & 1 & 0 & 0 & 0 & 1 & 0 & 7 \\
\hline A5 & $\begin{array}{r}\text { Ambidexterity Proportion Short } \\
\text { Term (T) Vs Mid Long Term (R) } \\
70 / 30\end{array}$ & 1 & 0 & 1 & 0 & 0 & 0 & 0 & 0 & 0 & 0 & 0 & 0 & 2 \\
\hline A6 & $\begin{array}{r}\text { Ambidexterity Proportion Short } \\
\text { Term (T) Vs Mid Long Term (R) } \\
80 / 20\end{array}$ & 0 & 0 & 0 & 0 & 0 & 0 & 0 & 1 & 1 & 1 & 0 & 1 & 4 \\
\hline A7 & $\begin{array}{r}\text { Ambidexterity Proportion Short } \\
\text { Term (T) Vs Mid Long Term (R) } \\
90 / 10\end{array}$ & 0 & 0 & 0 & 0 & 0 & 0 & 0 & 0 & 0 & 0 & 3 & 0 & 3 \\
\hline A8 & $\begin{array}{l}\text { Ambidexterity the } 30 \%(\mathrm{R}) \text { seems } \\
\text { to be much more Important than } \\
\text { the percentage per se }\end{array}$ & 1 & 0 & 0 & 0 & 0 & 0 & 0 & 0 & 0 & 0 & 0 & 0 & 1 \\
\hline A9 & $\begin{array}{r}\text { Exploitation First Year of my } \\
\text { Position was all about } \\
\text { Exploitation }\end{array}$ & 0 & 0 & 0 & 0 & 0 & 0 & 1 & 0 & 0 & 0 & 0 & 0 & 1 \\
\hline A10 & $\begin{array}{r}\text { Exploitation he Was not Good, } \\
\text { the Position Forced to Learn how } \\
\text { to be Good }\end{array}$ & 0 & 0 & 0 & 0 & 0 & 0 & 1 & 0 & 0 & 0 & 0 & 0 & 1 \\
\hline A11 & Exploitation Mid Term Focused In & 0 & 0 & 0 & 0 & 0 & 0 & 0 & 0 & 1 & 0 & 1 & 0 & 2 \\
\hline A12 & $\begin{array}{r}\text { Exploitation Second Year of my } \\
\text { Position was a liittle more R, } \\
80 \% \mathrm{~T} / 20 \% \mathrm{R}\end{array}$ & 0 & 0 & 0 & 0 & 0 & 0 & 1 & 0 & 0 & 0 & 0 & 0 & 1 \\
\hline A13 & $\begin{array}{r}\text { Exploration Good in } \\
\text { MediumTerm }\end{array}$ & 0 & 0 & 1 & 1 & 0 & 0 & 1 & 0 & 0 & 0 & 1 & 0 & 4 \\
\hline B & Ambidexterity Successor & 1 & 0 & 1 & 4 & 0 & 2 & 3 & 0 & 0 & 0 & $\mathbf{0}$ & 1 & 12 \\
\hline B1 & $\begin{array}{l}\text { Ambidexterity Dvision Directors } \\
\text { (HIGH DIRECTORS) } 70(\mathrm{~T}) / 30(\mathrm{R})\end{array}$ & 0 & 0 & 1 & 0 & 0 & 0 & 0 & 0 & 0 & 0 & 0 & 0 & 1 \\
\hline B2 & $\begin{array}{r}\text { Team Successor Ambidexterity } \\
\text { Desired Little bit More R than T } \\
(2 \mathrm{nd}(5 \mathrm{R} / 2 \mathrm{~T}) 1 \mathrm{st}(3.5 \mathrm{R} / 3.5 \mathrm{~T})) \\
(\text { HIGH DIRECTOR) }\end{array}$ & 0 & 0 & 0 & 0 & 0 & 0 & 0 & 0 & 0 & 0 & 0 & 1 & 1 \\
\hline B3 & $\begin{array}{l}\text { Team Successor Ambidexterity } \\
\text { Need to Move from R to T to be } \\
\text { (HIGH DIRECTOR) (AGREGAR) }\end{array}$ & 0 & 0 & 0 & 2 & 0 & 0 & 0 & 0 & 0 & 0 & 0 & 0 & 2 \\
\hline B4 & $\begin{array}{r}\text { Team Successor must be Good in } \\
\text { Operation (Exploitation) }\end{array}$ & 0 & 0 & 0 & 0 & 0 & 1 & 0 & 0 & 0 & 0 & 0 & 0 & 1 \\
\hline B5 & $\begin{array}{l}\text { Team Successor must be Good in } \\
\text { Operation (Exploitation) and also }\end{array}$ & 0 & 0 & 0 & 1 & 0 & 0 & 0 & 0 & 0 & 0 & 0 & 0 & 1 \\
\hline
\end{tabular}




\begin{tabular}{|c|c|c|c|c|c|c|c|c|c|c|c|c|c|c|}
\hline & $\begin{array}{r}\text { in } \mathrm{R} \text { but } \mathrm{T} \text { is important } \\
\text { (AGREGAR) }\end{array}$ & & & & & & & & & & & & & \\
\hline B6 & $\begin{array}{r}\text { Team Successor must have } \\
\text { Ambidexterity } 50 \mathrm{R} / 50 \mathrm{~T}\end{array}$ & 0 & 0 & 0 & 1 & 0 & 1 & 1 & 0 & 0 & 0 & 0 & 0 & 3 \\
\hline B7 & $\begin{array}{r}\text { Exploitation in this positions in } \\
\text { TEC Exploitation }(T) \text { is key more } \\
\text { than Exploration (R) (HIGH } \\
\text { DIRECTOR) }\end{array}$ & 0 & 0 & 0 & 0 & 0 & 0 & 1 & 0 & 0 & 0 & 0 & 0 & 1 \\
\hline B8 & $\begin{array}{r}\text { Strategy TEC Campus Director is } \\
\text { more Exploitation (T) than } \\
\text { Exploration (R) (HIGH DIRECTOR) }\end{array}$ & 1 & 0 & 0 & 0 & 0 & 0 & 0 & 0 & 0 & 0 & 0 & 0 & 1 \\
\hline B9 & $\begin{array}{r}\text { Team Successor must have } \\
\text { Ambidexterity } 80 T / 20 R\end{array}$ & 0 & 0 & 0 & 0 & 0 & 0 & 1 & 0 & 0 & 0 & 0 & 0 & 1 \\
\hline C & Ambidexterity High Directors & 5 & 0 & 7 & 5 & 1 & 3 & 2 & 1 & 6 & 2 & 1 & 1 & 34 \\
\hline C1 & $\begin{array}{r}\text { Ambidexterity Top of the Team } \\
\text { should be } R+\text { lower level more } T \\
\text { than } R+\text { high level directors } \\
\text { much more } R \text { than } T+R \text { should } \\
\text { increase with increasing } \\
\text { positiions + Campus director } \\
\text { more } R \text { than } T+\text { Successor more } \\
R \text { than } T \text { (HIGH DIRECTORS) } \\
\text { (AGREGAR) }\end{array}$ & 3 & 0 & 3 & 1 & 1 & 2 & 1 & 1 & 4 & 1 & 1 & 0 & 18 \\
\hline C2 & $\begin{array}{r}\text { Ambidexterity HIGH DIRECTORS } \\
\text { must be equilibrated } \mathrm{R} \text { and } \mathrm{T}\end{array}$ & 0 & 0 & 2 & 1 & 0 & 1 & 0 & 0 & 0 & 0 & 0 & 1 & 5 \\
\hline C3 & $\begin{array}{r}\text { Ambidexterity HEI need develope } \\
\text { more R directors }+ \text { TEC alson } \\
\text { needs more } \mathrm{R} \text { there are more } \mathrm{T} \\
\text { (AGREGADO) }\end{array}$ & 0 & 0 & 0 & 2 & 0 & 0 & 0 & 0 & 0 & 0 & 0 & 0 & 2 \\
\hline C4 & 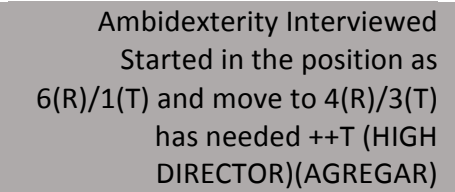 & 0 & 0 & 0 & 1 & 0 & 0 & 0 & 0 & 0 & 0 & 0 & 0 & 1 \\
\hline C5 & $\begin{array}{r}\text { Ambidexterity Rector (VERY HIGH } \\
\text { DIRECTORS) } 50(\mathrm{R}) / 50(\mathrm{~T}) \text { or } \\
30(\mathrm{~T}) / 70(\mathrm{R})\end{array}$ & 0 & 0 & 2 & 0 & 0 & 0 & 0 & 0 & 0 & 0 & 0 & 0 & 2 \\
\hline C6 & $\begin{array}{l}\text { Exploration HIGH DIRECTORS ++ } \\
\text { Exploitative (T) -- Expolrative (R) }\end{array}$ & 1 & 0 & 0 & 0 & 0 & 0 & 1 & 0 & 0 & 0 & 0 & 0 & 2 \\
\hline C7 & $\begin{array}{r}\text { Exploration Low level directors } \\
\text { much more T and high R (HIGH } \\
\text { DIRECTORS) }\end{array}$ & 0 & 0 & 0 & 0 & 0 & 0 & 0 & 0 & 1 & 0 & 0 & 0 & 1 \\
\hline C8 & $\begin{array}{l}\text { Exploration in Directors is } \\
\text { Needed Because Changing World }\end{array}$ & 0 & 0 & 0 & 0 & 0 & 0 & 0 & 0 & 1 & 1 & 0 & 0 & 2 \\
\hline C9 & $\begin{array}{l}\text { Strategy TEC Campus Director is } \\
\text { more Exploitation (T) than } \\
\text { Exploration (R) (HIGH DIRECTOR) }\end{array}$ & 1 & 0 & 0 & 0 & 0 & 0 & 0 & 0 & 0 & 0 & 0 & 0 & 1 \\
\hline $\mathrm{D}$ & Ambidexterity Team & 2 & $\mathbf{0}$ & $\mathbf{0}$ & 1 & 2 & 1 & 2 & 3 & 5 & 2 & 2 & 2 & 22 \\
\hline D1 & $\begin{array}{r}\text { Exploration How To Promote } \\
\text { Comparing Results Among } \\
\text { Members of the Team to Get the } \\
\text { Best Ideas }\end{array}$ & 0 & 0 & 0 & 0 & 0 & 1 & 0 & 0 & 0 & 0 & 0 & 0 & 1 \\
\hline D2 & Team Ambidexterity & 0 & 0 & 0 & 0 & 1 & 0 & 1 & 1 & 2 & 2 & 1 & 0 & 8 \\
\hline D3 & Team Ambidexterity ++R than $T$ & 0 & 0 & 0 & 0 & 0 & 0 & 0 & 0 & 1 & 0 & 0 & 0 & 1 \\
\hline D4 & Team Ambidexterity ++T and --R & 1 & 0 & 0 & 1 & 0 & 0 & 0 & 0 & 0 & 0 & 1 & 0 & 3 \\
\hline D5 & $\begin{array}{r}\text { Team Ambidexterity } 1(5 R / 2 T)^{*} \\
1(4 R / 3 T) 1(1 R / 6 T) 1(6 R / 1 T)\end{array}$ & 0 & 0 & 0 & 0 & 0 & 0 & 0 & 0 & 0 & 0 & 0 & 1 & 1 \\
\hline
\end{tabular}




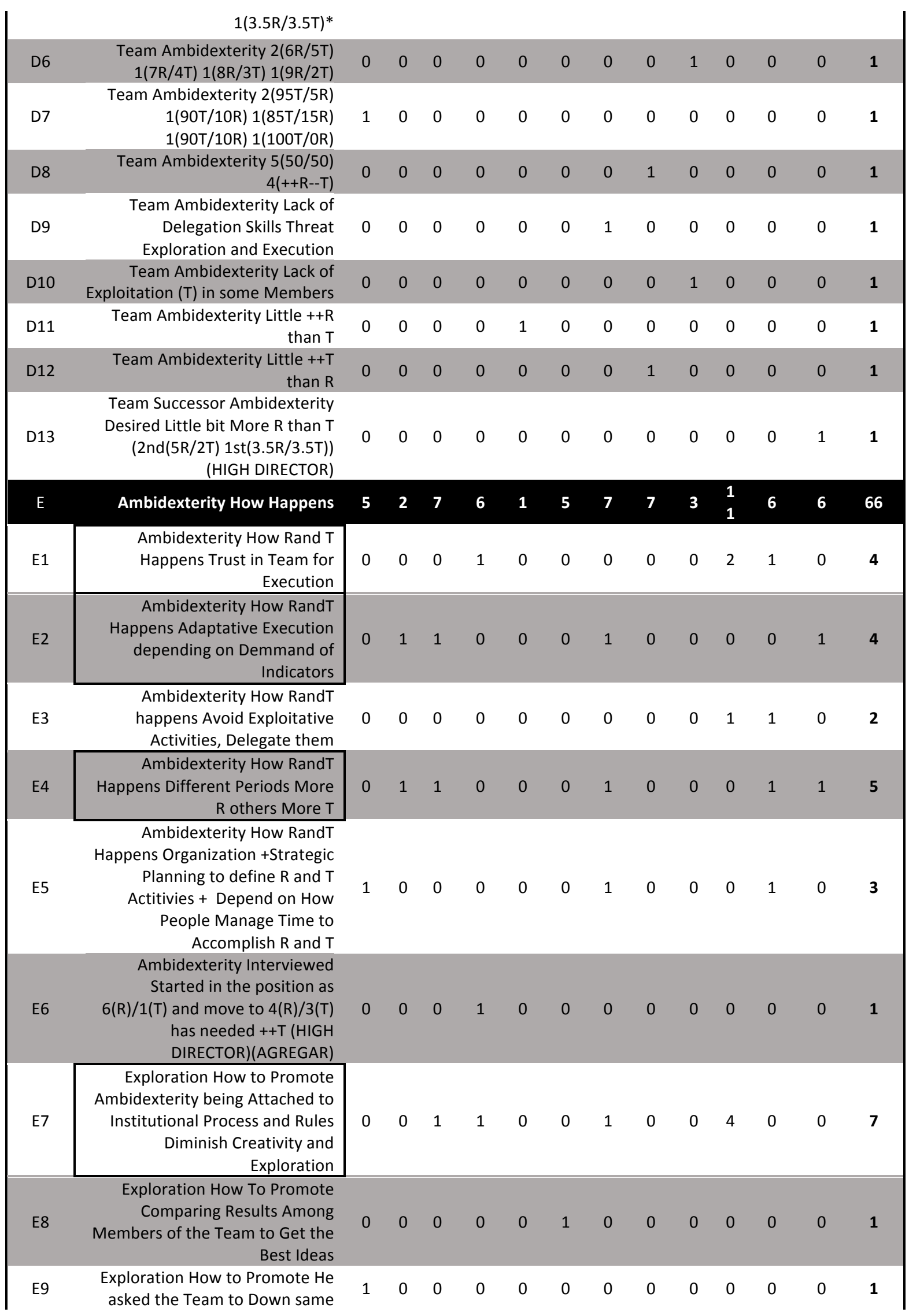




\begin{tabular}{|c|c|c|c|c|c|c|c|c|c|c|c|c|c|c|}
\hline \multicolumn{15}{|c|}{$\begin{array}{r}\text { Conversations that Promotes } \\
\text { Long Term View }\end{array}$} \\
\hline E10 & $\begin{array}{r}\text { Exploration How To Promote In } \\
\text { Team Motivating Looking Ahead } \\
\text { and Innovating + Investing time } \\
\text { in thinking + talk exlorative ideas } \\
\text { with team }\end{array}$ & 1 & 0 & 2 & 2 & 0 & 3 & 0 & 3 & 3 & 2 & 1 & 3 & 20 \\
\hline E11 & $\begin{array}{r}\text { Exploration How To Promote in } \\
\text { Free Time Activities likes Shower, } \\
\text { watching TV, in the Beach + } \\
\text { support areas doing their job + } \\
\text { delegating in a good team }\end{array}$ & 2 & 0 & 1 & 1 & 0 & 1 & 1 & 0 & 0 & 2 & 0 & 0 & 8 \\
\hline E12 & $\begin{array}{r}\text { Exploration How To Promote in } \\
\text { Team is Not Easy at all }\end{array}$ & 0 & 0 & 0 & 0 & 0 & 0 & 0 & 3 & 0 & 0 & 0 & 0 & 3 \\
\hline E13 & $\begin{array}{r}\text { Exploration How To Promote } \\
\text { Learnings from past Bosses }\end{array}$ & 0 & 0 & 1 & 0 & 0 & 0 & 0 & 0 & 0 & 0 & 0 & 0 & 1 \\
\hline E14 & $\begin{array}{r}\text { Exploration How To Promote Let } \\
\text { them Try }\end{array}$ & 0 & 0 & 0 & 0 & 0 & 0 & 0 & 0 & 0 & 0 & 1 & 0 & 1 \\
\hline E15 & $\begin{array}{r}\text { Exploration How To Promote } \\
\text { Planning and Keep Strategic } \\
\text { Projects with Team }\end{array}$ & 0 & 0 & 0 & 0 & 1 & 0 & 1 & 0 & 0 & 0 & 0 & 1 & 3 \\
\hline E16 & $\begin{array}{r}\text { Exploration How To Promote } \\
\text { Related to try being Out of } \\
\text { Comfort Zone }\end{array}$ & 0 & 0 & 0 & 0 & 0 & 0 & 0 & 1 & 0 & 0 & 0 & 0 & 1 \\
\hline E17 & $\begin{array}{r}\text { Exploration How To Promote the } \\
\text { Need of a Big Change }\end{array}$ & 0 & 0 & 0 & 0 & 0 & 0 & 1 & 0 & 0 & 0 & 0 & 0 & 1 \\
\hline $\mathrm{F}$ & Ambidexterity Structural & 1 & $\mathbf{0}$ & $\mathbf{0}$ & 1 & $\mathbf{0}$ & $\mathbf{0}$ & 3 & $\mathbf{0}$ & $\mathbf{0}$ & 1 & 1 & $\mathbf{0}$ & 6 \\
\hline F1 & $\begin{array}{l}\text { Ambidexterity How RandT } \\
\text { happens Avoid Exploitative } \\
\text { Activities, Delegate them }\end{array}$ & 1 & 0 & 0 & 0 & 0 & 0 & 0 & 0 & 0 & 1 & 1 & 0 & 2 \\
\hline F2 & $\begin{array}{r}\text { Ambidexterity Structural Example } \\
\text { (AGREGAR) }\end{array}$ & 0 & 0 & 0 & 1 & 0 & 0 & 0 & 0 & 0 & 0 & 0 & 0 & 1 \\
\hline F3 & $\begin{array}{r}\text { Strategy Interviewed Not Depent } \\
\text { in DDC the Strategy of Career but } \\
\text { Solidity of Processes and Seize } \\
\text { Strengths of Region }\end{array}$ & 0 & 0 & 0 & 0 & 0 & 0 & 2 & 0 & 0 & 0 & 0 & 0 & 2 \\
\hline F4 & $\begin{array}{r}\text { Team Ambidexterity Lack of } \\
\text { Delegation Skills Threat } \\
\text { Exploration and Execution } \\
\text { (Borrar) }\end{array}$ & 0 & 0 & 0 & 0 & 0 & 0 & 1 & 0 & 0 & 0 & 0 & 0 & 1 \\
\hline G & Ambidexterity Contextual & 3 & 4 & 3 & 2 & 1 & 2 & 9 & 0 & 0 & 4 & 3 & 3 & 34 \\
\hline G1 & $\begin{array}{r}\text { Ambidexterity How Rand T } \\
\text { Happens Trust in Team for } \\
\text { Execution }\end{array}$ & 0 & 0 & 0 & 1 & 0 & 0 & 0 & 0 & 0 & 2 & 1 & 0 & 4 \\
\hline $\mathrm{G} 2$ & $\begin{array}{r}\text { Ambidexterity How RandT } \\
\text { Happens Different Periods More } \\
\text { R others More T }\end{array}$ & 0 & 1 & 1 & 0 & 0 & 0 & 1 & 0 & 0 & 0 & 1 & 1 & 5 \\
\hline G3 & $\begin{array}{r}\text { Ambidexterity How RandT } \\
\text { Happens Adaptative Execution } \\
\text { depending on Demmand of } \\
\text { Indicators }\end{array}$ & 0 & 1 & 1 & 0 & 0 & 0 & 1 & 0 & 0 & 0 & 0 & 1 & 4 \\
\hline G4 & $\begin{array}{r}\text { Ambidexterity Interviewed } \\
\text { Dynamic Depending Needs, bot } \\
\text { more }(T) \text { than }(R)\end{array}$ & 0 & 2 & 0 & 0 & 0 & 0 & 0 & 0 & 0 & 0 & 0 & 0 & 2 \\
\hline G5 & $\begin{array}{r}\text { Exploitation First Year of my } \\
\text { Position was all about } \\
\text { Exploitation }\end{array}$ & 0 & 0 & 0 & 0 & 0 & 0 & 1 & 0 & 0 & 0 & 0 & 0 & 1 \\
\hline G6 & Exploitation Second Year of my & 0 & 0 & 0 & 0 & 0 & 0 & 1 & 0 & 0 & 0 & 0 & 0 & 1 \\
\hline
\end{tabular}




\begin{tabular}{|c|c|c|c|c|c|c|c|c|c|c|c|c|c|c|}
\hline & $\begin{array}{r}\text { Position was a liittle more } R, \\
80 \% \mathrm{~T} / 20 \% R\end{array}$ & & & & & & & & & & & & & \\
\hline G7 & $\begin{array}{r}\text { Exploration How To Promote } \\
\text { Comparing Results Among } \\
\text { Members of the Team to Get the } \\
\text { Best Ideas }\end{array}$ & 0 & 0 & 0 & 0 & 0 & 1 & 0 & 0 & 0 & 0 & 0 & 0 & 1 \\
\hline G8 & $\begin{array}{r}\text { Exploration How to Promote He } \\
\text { asked the Team to Down same } \\
\text { Conversations that Promotes } \\
\text { Long Term View }\end{array}$ & 1 & 0 & 0 & 0 & 0 & 0 & 0 & 0 & 0 & 0 & 0 & 0 & 1 \\
\hline G9 & $\begin{array}{r}\text { Exploration How To Promote in } \\
\text { Free Time Activities likes Shower, } \\
\text { watching TV, in the Beach + } \\
\text { support areas doing their job + } \\
\text { delegating in a good team }\end{array}$ & 2 & 0 & 1 & 1 & 0 & 1 & 1 & 0 & 0 & 2 & 0 & 0 & 8 \\
\hline G10 & $\begin{array}{r}\text { Exploration How To Promote Let } \\
\text { them Try }\end{array}$ & 0 & 0 & 0 & 0 & 0 & 0 & 0 & 0 & 0 & 0 & 1 & 0 & 1 \\
\hline G11 & $\begin{array}{r}\text { Exploration How To Promote } \\
\text { Planning and Keep Strategic } \\
\text { Projects with Team }\end{array}$ & 0 & 0 & 0 & 0 & 1 & 0 & 1 & 0 & 0 & 0 & 0 & 1 & 3 \\
\hline G12 & $\begin{array}{r}\text { Strategy Interviewed Not Depent } \\
\text { in DDC the Strategy of Career but } \\
\text { Solidity of Processes and Seize } \\
\text { Strengths of Region }\end{array}$ & 0 & 0 & 0 & 0 & 0 & 0 & 2 & 0 & 0 & 0 & 0 & 0 & 2 \\
\hline G13 & $\begin{array}{r}\text { Strategy Interviewed say } \\
\text { Processes must be Solid in order } \\
\text { to Advance in Strategy }\end{array}$ & 0 & 0 & 0 & 0 & 0 & 0 & 1 & 0 & 0 & 0 & 0 & 0 & 1 \\
\hline $\mathrm{H}$ & Ambidexterity Threats & 1 & 1 & 2 & 3 & 0 & 0 & 3 & 4 & $\mathbf{0}$ & 6 & $\mathbf{0}$ & 2 & 22 \\
\hline $\mathrm{H} 1$ & $\begin{array}{r}\text { Exploitation in Excess make Lack } \\
\text { of Exploration in Strategy }\end{array}$ & 1 & 0 & 0 & 0 & 0 & 0 & 0 & 0 & 0 & 1 & 0 & 1 & 3 \\
\hline $\mathrm{H} 2$ & $\begin{array}{r}\text { Exploration Indicators and } \\
\text { Innovation Affect Day to Day (T) } \\
\text { indicators }\end{array}$ & 0 & 0 & 0 & 0 & 0 & 0 & 0 & 0 & 0 & 0 & 0 & 1 & 1 \\
\hline $\mathrm{H} 3$ & $\begin{array}{r}\text { Exploration Threat Neg Lack of } \\
\text { Time }\end{array}$ & 0 & 0 & 0 & 0 & 0 & 0 & 0 & 2 & 0 & 0 & 0 & 0 & 2 \\
\hline $\mathrm{H} 4$ & $\begin{array}{r}\text { Exploration Threat Neg Too Much } \\
\text { Work in Parallel }\end{array}$ & 0 & 0 & 0 & 1 & 0 & 0 & 0 & 2 & 0 & 0 & 0 & 0 & 3 \\
\hline $\mathrm{H} 5$ & $\begin{array}{r}\text { Exploration Threat Not to be } \\
\text { Good in Delegation }\end{array}$ & 0 & 0 & 0 & 0 & 0 & 0 & 1 & 0 & 0 & 0 & 0 & 0 & 1 \\
\hline $\mathrm{H} 6$ & $\begin{array}{r}\text { Exploration How to Promote } \\
\text { Ambidexterity being Attached to } \\
\text { Institutional Process and Rules } \\
\text { Diminish Creativity and } \\
\text { Exploration }\end{array}$ & 0 & 0 & 1 & 1 & 0 & 0 & 1 & 0 & 0 & 4 & 0 & 0 & 7 \\
\hline $\mathrm{H} 7$ & $\begin{array}{r}\text { Ambidexterity Focused in } \\
\text { Exploitative KPI can diminish the } \\
\text { Exploration (T) }\end{array}$ & 0 & 1 & 1 & 1 & 0 & 0 & 1 & 0 & 0 & 1 & 0 & 0 & 5 \\
\hline 1 & Ambidexterity General & 7 & 0 & 4 & 3 & 3 & 4 & 1 & 2 & 2 & 9 & 3 & 4 & 42 \\
\hline $\mid 1$ & $\begin{array}{r}\text { Ambidexterity I wish I could } \\
\text { Spend More Time in Exploration } \\
(\mathrm{R})\end{array}$ & 1 & 0 & 2 & 0 & 0 & 0 & 0 & 0 & 0 & 1 & 0 & 0 & 4 \\
\hline 12 & $\begin{array}{r}\text { Exploration could be Developed } \\
\text { with Training } \\
\end{array}$ & 2 & 0 & 0 & 1 & 0 & 0 & 0 & 0 & 0 & 0 & 1 & 0 & 4 \\
\hline 13 & Exploration Example & 3 & 0 & 2 & 1 & 3 & 3 & 1 & 2 & 1 & 6 & 0 & 1 & 23 \\
\hline 14 & $\begin{array}{r}\text { Exploration in Directors is } \\
\text { Needed Because Changing World } \\
\text { and Fast Pace in Environment }\end{array}$ & 1 & 0 & 0 & 1 & 0 & 0 & 0 & 0 & 1 & 1 & 0 & 1 & 5 \\
\hline
\end{tabular}




\begin{tabular}{|c|c|c|c|c|c|c|c|c|c|c|c|c|c|c|}
\hline 15 & $\begin{array}{r}\text { Exploitation In Favor of } \\
\text { Exploitation Indicators Pay for } \\
\text { Exploration Possibillities }\end{array}$ & 0 & 0 & 0 & 0 & 0 & 1 & 0 & 0 & 0 & 1 & 2 & 2 & 6 \\
\hline J & Ambidexterity Boss & $\mathbf{0}$ & 0 & 1 & 1 & 0 & 0 & 1 & $\mathbf{0}$ & 4 & 1 & 0 & 1 & 9 \\
\hline J1 & Boss Ambidexterity 100T/0R & 0 & 0 & 0 & 0 & 0 & 0 & 1 & 0 & 0 & 0 & 0 & 0 & 1 \\
\hline $\mathrm{J} 2$ & $\begin{array}{l}\text { Boss Ambidexterity } 40 \mathrm{R} / 60 \mathrm{~T} \\
\text { according to Interviewed }\end{array}$ & 0 & 0 & 0 & 0 & 0 & 0 & 0 & 0 & 1 & 0 & 0 & 0 & 1 \\
\hline J3 & $\begin{array}{l}\text { Boss Ambidexterity 50R/50T } \\
\text { according to Interviewed }\end{array}$ & 0 & 0 & 0 & 1 & 0 & 0 & 0 & 0 & 3 & 1 & 0 & 0 & 5 \\
\hline J4 & Boss Ambidexterity 70R/30T & 0 & 0 & 1 & 0 & 0 & 0 & 0 & 0 & 0 & 0 & 0 & 0 & 1 \\
\hline J5 & $\begin{array}{l}\text { Boss Ambidexterity } 95 \mathrm{R} / 05 \mathrm{~T} \\
\text { according to Interviewed }\end{array}$ & 0 & 0 & 0 & 0 & 0 & 0 & 0 & 0 & 0 & 0 & 0 & 1 & 1 \\
\hline & TOTAL & 28 & 9 & 32 & 27 & 9 & 18 & 36 & 18 & 22 & $\begin{array}{l}3 \\
9\end{array}$ & 23 & 21 & 281 \\
\hline
\end{tabular}

Table 20. Code and Extended Codes for Ambidexterity Theme at Interviewee detailed.

\begin{tabular}{|c|c|c|}
\hline$\#$ & Code & MENTIONS \\
\hline A & Ambidexterity Interviewed & 34 \\
\hline A1 & Ambidexterity I wish I could Spend More Time in Exploration (R) & 4 \\
\hline A2 & Ambidexterity Interviewed ++5R--2T & 2 \\
\hline A3 & Ambidexterity Interviewed Dynamic Depending Needs, bot more $(T)$ than $(R)$ & 2 \\
\hline A4 & Ambidexterity Interviewed Exploration More Mid and Long Term Person (R) & 7 \\
\hline A5 & Ambidexterity Proportion Short Term (T) Vs Mid Long Term (R) 70/30 & 2 \\
\hline A6 & Ambidexterity Proportion Short Term (T) Vs Mid Long Term (R) 80/20 & 4 \\
\hline A7 & Ambidexterity Proportion Short Term (T) Vs Mid Long Term (R) 90/10 & 3 \\
\hline A8 & $\begin{array}{r}\text { Ambidexterity the } 30 \%(R) \text { seems to be much more Important than the } \\
\text { percentage per se }\end{array}$ & 1 \\
\hline A9 & Exploitation First Year of my Position was all about Exploitation & 1 \\
\hline A10 & Exploitation he Was not Good, the Position Forced to Learn how to be Good & 1 \\
\hline A11 & Exploitation Mid Term Focused In & 2 \\
\hline A12 & Exploitation Second Year of my Position was a liittle more R, $80 \% \mathrm{~T} / 20 \% \mathrm{R}$ & 1 \\
\hline A13 & Exploration Good in MediumTerm & 4 \\
\hline B & Ambidexterity Successor & 12 \\
\hline B1 & Ambidexterity Dvision Directors (HIGH DIRECTORS) $70(T) / 30(R)$ & 1 \\
\hline B2 & $\begin{array}{r}\text { Team Successor Ambidexterity Desired Little bit More R than T (2nd(5R/2T) } \\
1 \text { st(3.5R/3.5T)) (HIGH DIRECTOR) }\end{array}$ & 1 \\
\hline B3 & $\begin{array}{r}\text { Team Successor Ambidexterity Need to Move from } R \text { to } T \text { to be (HIGH } \\
\text { DIRECTOR) (AGREGAR) }\end{array}$ & 2 \\
\hline B4 & Team Successor must be Good in Operation (Exploitation) & 1 \\
\hline B5 & $\begin{array}{r}\text { Team Successor must be Good in Operation (Exploitation) and also in R but } T \text { is } \\
\text { important (AGREGAR) }\end{array}$ & 1 \\
\hline B6 & Team Successor must have Ambidexterity 50R/50T & 3 \\
\hline
\end{tabular}


Strategy TEC Campus Director is more Exploitation (T) than Exploration (R)

B9

(HIGH DIRECTOR)

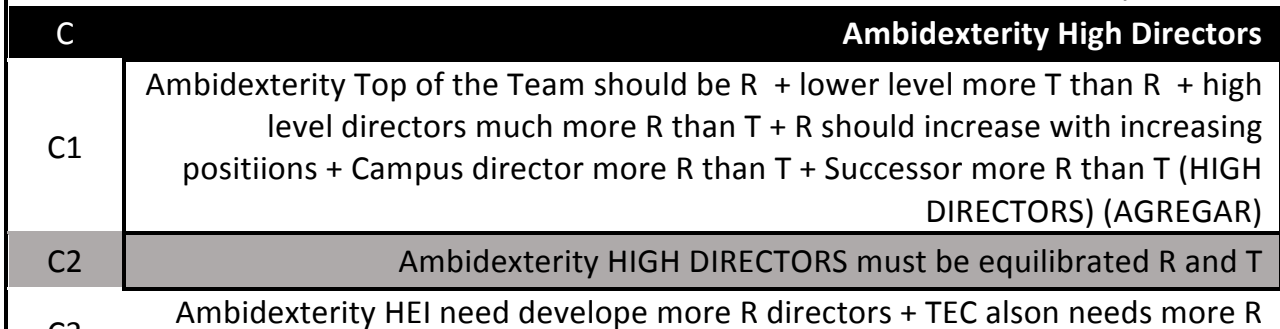

C3 Ambidexterity HEI need develope more R directors + TEC alson needs more $R$
there are more T (AGREGADO)

C4 Ambidexterity Interviewed Started in the position as $6(R) / 1(T)$ and move to

4(R)/3(T) has needed ++T (HIGH DIRECTOR)(AGREGAR)

C5 Ambidexterity Rector (VERY HIGH DIRECTORS) $50(\mathrm{R}) / 50(\mathrm{~T})$ or 30(T)/70(R)

C6 Exploration HIGH DIRECTORS ++ Exploitative (T) -- Expolrative (R)

C7 Exploration Low level directors much more T and high R (HIGH DIRECTORS)

C8 Exploration in Directors is Needed Because Changing World

C9 Strategy TEC Campus Director is more Exploitation (T) than Exploration (R)

(HIGH DIRECTOR)

D

Ambidexterity Team

$\mathrm{D}$

Exploration How To Promote Comparing Results Among Members of the Team

\begin{tabular}{|c|c|}
\hline D2 & Team Ambidexterity \\
\hline D3 & Team Ambidexterity $++R$ than $T$ \\
\hline D4 & Team Ambidexterity ++T and --R \\
\hline
\end{tabular}

to Get the Best Ideas

$\begin{array}{rr}\text { D5 } & \text { Team Ambidexterity } 1(5 R / 2 T)^{*} 1(4 R / 3 T) 1(1 R / 6 T) 1(6 R / 1 T) 1(3.5 R / 3.5 T)^{*} \\ \text { D6 } & \text { Team Ambidexterity 2(6R/5T) } 1(7 R / 4 T) 1(8 R / 3 T) 1(9 R / 2 T)\end{array}$

D7 Team Ambidexterity 2(95T/5R) 1(90T/10R) 1(85T/15R) 1(90T/10R) 1(100T/OR) 1

D8 Team Ambidexterity 5(50/50) 4(++R--T) 1

D9 Team Ambidexterity Lack of Delegation Skills Threat Exploration and Execution $\mathbf{1}$

D10 Team Ambidexterity Lack of Exploitation $(T)$ in some Members 1

D11 Team Ambidexterity Little ++R than T 1

D12 Team Ambidexterity Little ++T than R 1

$\begin{array}{rrr}\text { D13 Team Successor Ambidexterity Desired Little bit More R than T (2nd(5R/2T) } & \mathbf{1}\end{array}$

\begin{tabular}{|c|c|c|}
\hline $\mathrm{E}$ & Ambidexterity How Happens & 66 \\
\hline E1 & Ambidexterity How Rand T Happens Trust in Team for Execution & 4 \\
\hline E2 & $\begin{array}{r}\text { Ambidexterity How RandT Happens Adaptative Execution depending on } \\
\text { Demmand of Indicators }\end{array}$ & 4 \\
\hline E3 & $\begin{array}{r}\text { Ambidexterity How RandT happens Avoid Exploitative Activities, Delegate } \\
\text { them }\end{array}$ & 2 \\
\hline E4 & Ambidexterity How RandT Happens Different Periods More R others More T & 5 \\
\hline E5 & $\begin{array}{l}\text { Ambidexterity How RandT Happens Organization +Strategic Planning to define } \\
R \text { and T Actitivies + Depend on How People Manage Time to Accomplish R and }\end{array}$ & 3 \\
\hline E6 & $\begin{array}{l}\text { Ambidexterity Interviewed Started in the position as } 6(\mathrm{R}) / 1(\mathrm{~T}) \text { and move to } \\
\qquad 4(\mathrm{R}) / 3(\mathrm{~T}) \text { has needed }++\mathrm{T}(\mathrm{HIGH} \text { DIRECTOR)(AGREGAR) }\end{array}$ & 1 \\
\hline
\end{tabular}




\begin{tabular}{|c|c|c|}
\hline E7 & $\begin{array}{r}\text { Exploration How to Promote Ambidexterity being Attached to Institutional } \\
\text { Process and Rules Diminish Creativity and Exploration }\end{array}$ & 7 \\
\hline E8 & $\begin{array}{r}\text { Exploration How To Promote Comparing Results Among Members of the Team } \\
\text { to Get the Best Ideas }\end{array}$ & 1 \\
\hline E9 & $\begin{array}{r}\text { Exploration How to Promote He asked the Team to Down same Conversations } \\
\text { that Promotes Long Term View }\end{array}$ & 1 \\
\hline E10 & $\begin{array}{r}\text { Exploration How To Promote In Team Motivating Looking Ahead and } \\
\text { Innovating + Investing time in thinking + talk exlorative ideas with team }\end{array}$ & 20 \\
\hline E11 & $\begin{array}{l}\text { Exploration How To Promote in Free Time Activities likes Shower, watching TV, } \\
\text { in the Beach + support areas doing their job + delegating in a good team }\end{array}$ & 8 \\
\hline E12 & Exploration How To Promote in Team is Not Easy at all & 3 \\
\hline E13 & Exploration How To Promote Learnings from past Bosses & 1 \\
\hline E14 & Exploration How To Promote Let them Try & 1 \\
\hline E15 & Exploration How To Promote Planning and Keep Strategic Projects with Team & 3 \\
\hline E16 & Exploration How To Promote Related to try being Out of Comfort Zone & 1 \\
\hline E17 & Exploration How To Promote the Need of a Big Change & 1 \\
\hline $\mathrm{F}$ & Ambidexterity Structural & 6 \\
\hline F1 & $\begin{array}{r}\text { Ambidexterity How RandT happens Avoid Exploitative Activities, Delegate } \\
\text { them }\end{array}$ & 2 \\
\hline F2 & Ambidexterity Structural Example (AGREGAR) & 1 \\
\hline F3 & $\begin{array}{r}\text { Strategy Interviewed Not Depent in DDC the Strategy of Career but Solidity of } \\
\text { Processes and Seize Strengths of Region }\end{array}$ & 2 \\
\hline F4 & $\begin{array}{r}\text { Team Ambidexterity Lack of Delegation Skills Threat Exploration and Execution } \\
\text { (Borrar) }\end{array}$ & 1 \\
\hline G & Ambidexterity Contextual & 34 \\
\hline G1 & Ambidexterity How Rand T Happens Trust in Team for Execution & 4 \\
\hline G2 & Ambidexterity How RandT Happens Different Periods More R others More T & 5 \\
\hline G3 & $\begin{array}{r}\text { Ambidexterity How RandT Happens Adaptative Execution depending on } \\
\text { Demmand of Indicators }\end{array}$ & 4 \\
\hline G4 & Ambidexterity Interviewed Dynamic Depending Needs, bot more $(T)$ than $(R)$ & 2 \\
\hline G5 & Exploitation First Year of my Position was all about Exploitation & 1 \\
\hline G6 & Exploitation Second Year of my Position was a liittle more R, $80 \% \mathrm{~T} / 20 \% \mathrm{R}$ & 1 \\
\hline G7 & $\begin{array}{r}\text { Exploration How To Promote Comparing Results Among Members of the Team } \\
\text { to Get the Best Ideas }\end{array}$ & 1 \\
\hline G8 & $\begin{array}{r}\text { Exploration How to Promote He asked the Team to Down same Conversations } \\
\text { that Promotes Long Term View }\end{array}$ & 1 \\
\hline G9 & $\begin{array}{r}\text { Exploration How To Promote in Free Time Activities likes Shower, watching TV, } \\
\text { in the Beach + support areas doing their job + delegating in a good team }\end{array}$ & 8 \\
\hline G10 & Exploration How To Promote Let them Try & 1 \\
\hline G11 & Exploration How To Promote Planning and Keep Strategic Projects with Team & 3 \\
\hline G12 & $\begin{array}{r}\text { Strategy Interviewed Not Depent in DDC the Strategy of Career but Solidity of } \\
\text { Processes and Seize Strengths of Region }\end{array}$ & 2 \\
\hline G13 & $\begin{array}{r}\text { Strategy Interviewed say Processes must be Solid in order to Advance in } \\
\text { Strategy }\end{array}$ & 1 \\
\hline $\mathrm{H}$ & $\begin{array}{ll}\text { Ambidexterity Threats } \\
\end{array}$ & 22 \\
\hline $\mathrm{H} 1$ & Exploitation in Excess make Lack of Exploration in Strategy & 3 \\
\hline $\mathrm{H} 2$ & Exploration Indicators and Innovation Affect Day to Day $(\mathrm{T})$ indicators & 1 \\
\hline $\mathrm{H} 3$ & Exploration Threat Neg Lack of Time & 2 \\
\hline $\mathrm{H} 4$ & Exploration Threat Neg Too Much Work in Parallel & 3 \\
\hline
\end{tabular}




\begin{tabular}{|c|c|c|}
\hline H5 & Exploration Threat Not to be Good in Delegation & 1 \\
\hline H6 & $\begin{array}{r}\text { Exploration How to Promote Ambidexterity being Attached to Institutional } \\
\text { Process and Rules Diminish Creativity and Exploration }\end{array}$ & 7 \\
\hline $\mathrm{H7}$ & Ambidexterity Focused in Exploitative KPI can diminish the Exploration (T) & 5 \\
\hline I & Ambidexterity General & 42 \\
\hline 11 & Ambidexterity I wish I could Spend More Time in Exploration (R) & 4 \\
\hline 12 & Exploration could be Developed with Training & 4 \\
\hline 13 & Exploration Example & 23 \\
\hline 14 & $\begin{array}{r}\text { Exploration in Directors is Needed Because Changing World and Fast Pace in } \\
\text { Environment }\end{array}$ & 5 \\
\hline 15 & Exploitation In Favor of Exploitation Indicators Pay for Exploration Possibillities & 6 \\
\hline $\mathrm{J}$ & Ambidexterity Boss & 9 \\
\hline $\mathrm{J} 1$ & Boss Ambidexterity 100T/0R & 1 \\
\hline $\mathrm{J} 2$ & Boss Ambidexterity 40R/60T according to Interviewed & 1 \\
\hline J3 & Boss Ambidexterity 50R/50T according to Interviewed & 5 \\
\hline J4 & Boss Ambidexterity 70R/30T & 1 \\
\hline $\mathrm{J} 5$ & Boss Ambidexterity 95R/05T according to Interviewed & 1 \\
\hline & TOTAL & 281 \\
\hline
\end{tabular}

Table 21. Code and Extended Codes for Ambidexterity Theme. 


\section{FIGURES.}

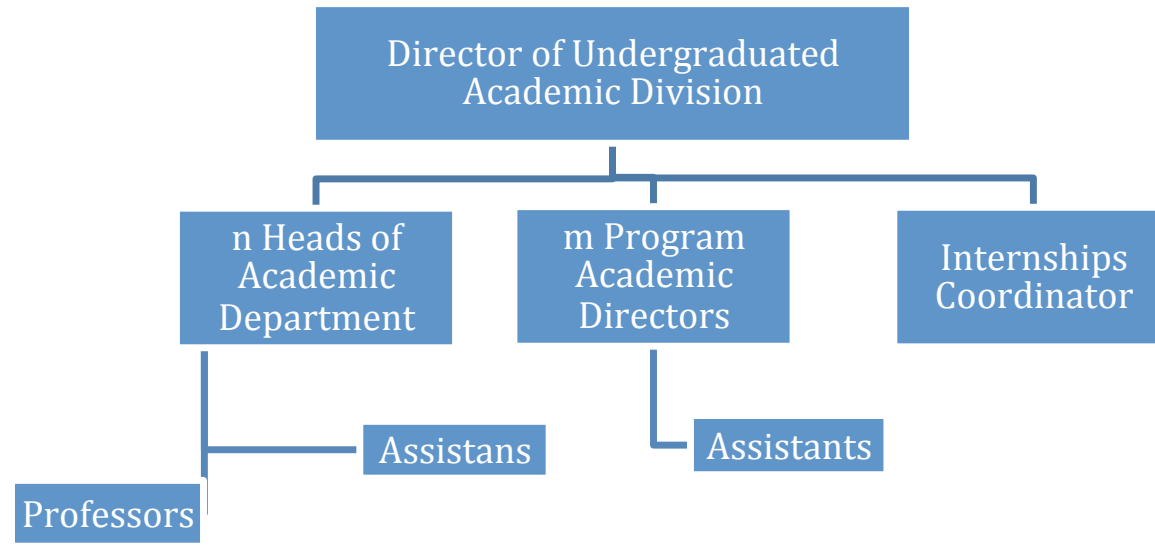

Source: own elaboration, based on Organizational structure, Tecnológico de Monterrey Campus Sonora Norte, 2015.

Figure 1. Campus Sonora Norte Undergraduated Academic Division organizational chart. 


\section{REFERENCES.}

Ambrosini, V., \& Bowman, C. (2009). What are dynamic capabilities and are they a useful construct in strategic management? International Journal of Management Reviews , 11 (1), 29-49.

Ambrosini, V., Bowman, C., \& Collier, N. (2009). Dynamic Capabilities: An Exploration of How Firms Renew their Resource Base. British Journal of Management, 20, S9-S24.

Amit, R., \& Schoemaker, P. J. (1993). Strategic Assets and Organizational Rent. Strategic Management Journal , 14 (1), 33-46.

Argyris, C. (1966). Interpersonal Barriers in Decision Making. Harvard Business Review , 59-95.

Ariely, D., \& Norton, M. I. (2011). From thinking too little to thinking too much: a continuum of decision making. Cognitive Science, 2 (1), 39.46.

Atmosudirdjo, P. (1987). Beberapa Pandangan Umum Tentang Pengambilan Keputusan. Jakarta.

Balaton, K. (2007). Organizational Strategies and Structures Following the System Turnaround. Budapest, Hungary: Akadémiai Kiadó.

Barber, C. S., \& Tietje, B. C. (2004). Competency requirements for managerial development in manufacturing, assembly, and/or material processing functions. Journal of Management Development , 23 (6), 596-607.

Barney, J. (1991). Firm Resources and Sustained Competitive Advantage. Journal of Management, 17 (1), 99-120.

Barney, J. (1986). Strategic Factor Markets: Expectations, Luck \& Business Strategy. Management Science, 32 (10), 1231-1241.

Barrett, S. E. (2010, August-September). Competitive Intelligence: Significance in Higher Education. World Future Review. , 26-30.

Baxter, P., \& Jack, S. (2008). Qualitative Case Study Methodology: Study Design and Implementation for Novice Researchers. The Qualitative Report, 13 (4), 544-559.

Berardi , A., \& Blackmore, C. (2006). Introducing Environmental Decision Making . (Vol. 1). GB: Open University Worldwide .

Bolden, R., Petrov , G., \& Gosling, J. (2007). Developing Collective Leadership in Higher Education. Final Report for the Leadership Foundation for Higher Education. Exeter: Centre for Leadership Studies.

Boldt, D. B. (1991). University strategic management: a businessman's view". International Journal of Education Management, 5 (5).

Boroski, J., \& Dryer , L. (1996). Key competencies for a transformed human resources organization: Results from a field study. Human Resource Management , 35 (3), 383403. 
Bossidy, L., Charam, R., \& Burck, C. (2002). Execution: The Discipline of Getting Things Done (Vol. 1). Crown Business.

Bowen , W., \& Shapiro, H. (1998). Universities and their leadership. New Jersey: Princeton University Press.

Bowman , R. F. (2002). The Real Work of the Deparment Chair. The Clearing House, 75 (3), 158-162.

Boyatzis, R. E. (1998). Transforming Qualitative Information. California, USA: SAGE Publications.

Brahma, S. S., \& Chakraborty, H. (2011). From Industry to Firm Resources: ResourceBased View of Competitive Advantage. The IUP Journal of Business Strategy, VIII, 16.

Burgelman, R. A. (1991). Intraorganizational Ecology of Strategy Making and Organizational Adaptation: Theory and Field Research. Organization Science , 2, 239262.

Camacho-Ruelas, G. (2012). Exploring the role of marketing in corporate sustainability: A case study. Tecnológico de Monterrey. Monterrey: Faculty of EGADE Business School.

Campbell, D. T., \& Fiske, D. W. (1959). Convergent and discriminant validation by the multitrait-multimethod matrix. Psychological Bulletin , 56, 81-105.

Casares, J., Dickson, D. A., Hannigan, T., Hinton, J., \& Phelps, A. (2015). The Future of Teaching and Learning in Higher Education. Retrieved January 6th, 2016, from https://www.rit.edu/provost/sites/rit.edu.provost/files/future_of_teaching_and_lea rning_reportv13.pdf

Cecil, E. A., \& Jundgren, E. F. (1974). An Analysis of Individual Decision MAking Benahior Using a Laboratory Setting. 600-605.

Choban, M. C., Choban, G. M., \& Choban, D. (2008). Strategic Planning and Decision Making in Higher Education:What Gets Attention and What Doesn't. Assessment Update Progress, Trends, and Practices in Higher Education, 20 (2), 1-14.

Clayton, M. J. (1997). Delphi: A technique to harness expert opinion for critical decision tasks in education. Educational Psychology. 97 (17), 373-386.

Cohen, W. A. (2008). En Clase con Drucker. (A. d. Londoño, Trans.) Colombia: Grupo Editorial Norma.

Cowburn, S. (2005). Strategic Planning in Higher Education: Fact or Fiction? Perspective, 9 (4), 103-109.

Creswell, J. W. (2003). Research Design: Qualitative, Quantitative and Mixed Methods Approaches. Thousand Oaks, CA: SAGE Publications.

Creswell, J. W. (2013). Qualitative Inquiry and Research Design: Choosing Among Five Approaches (3rd. ed.). USA: SAGE Publications. 
Daniels, K., \& Bailey, A. (1999). Strategy Development Processes and Participation in Decision Making: predictors of role stressors and job satisfaction. Journal of Applied Management Studies , 8 (1), 27-42.

Dawson, M. D., \& Brucker, P. S. (2001). The utility of the Delphi method in MFT research. American Journal of Family Therapy. , 125-140.

Dearlove, J. (1998). The Deadly Dull Issue of University Administration? Good Governance, Managerialism, and Organising Academic Work. Higher Education Policy , 11, 59-79.

Denzin, N. K. (1978). The Research Act: A Theoretical Introduction to Sociological Methods. New York: McGraw-Hill.

Dhliwayo, K. (2014). The Internal Customers Perceptions of A Multi-Campus University System In Zimbabwe. A Case of Great Zimbabwe University. INTERNATIONAL JOURNAL OF SCIENTIFIC \& TECHNOLOGY RESEARCH , 3 (2), 324330.

Dierickx, I., \& Cool, K. (1989). Asset Stock Accumulation and Sustainability of Competitive Advantage. Management Science , 35 (12), 1504-1513.

Diesing, P. (1971). Patterns of Discovery in the Social Sciences. 1971: Aldine-Atherton.

Dijksterhuis, A., Bos, M. W., Nordgren, L. F., \& Van Baaren, R. B. (2006). On Making the Right Choice: The Deliberation-Without-Attention Effect. Science , 1005-1007.

Dooris, M. J., Kelley, J. M., \& Trainer, J. F. (2002). Strategic Planning in Higher Education. New Directions in Higher Education .

Doz, Y., \& Kosonen, M. (2008). The Dynamics of Strategic Agility: NOKIA'S ROLLERCOASTER EXPERIENCE. CALIFORNIA MANAGEMENT REVIEW , 50 (3), 95118.

Drucker, P. F. (1967). The Effective Decision. Harvard Business Review , I (45), 92-98.

Drucker, P. (1954). The Practice of Management. New York: Harper \& Row Publishers.

Duncan, R. B. (1976). The ambidextrous organization: Designing dual structures for innovation. The management of organization , 1, 167-188.

Easterby-Smith, M., Thorpe, R., \& Jackson, P. R. (2008). Management Research. London: SAGE Pulications.

Edwards, W. (1954). The Theory of Decision Making. Psychological Bulletin , 380-417.

Eisenhardt, K. M. (1999). Strategy as Strategic Decision-making. Sloan Management Review , 79 (8).

Eisenhardt, K. M., \& Martin, J. A. (2000). Dynamic Capabilities: What are they? Strategic Management Journal , 21, 1105-1121.

Eisenhardt, K. M., \& Zbaracki, M. J. (1992). Strategic Decision Making. Strategic Management Journal , 13, 17-37. 
Ellonen, H.-K., Jantunen, A., \& Kuivalainen, O. (2011). The Role of Dynamic Capabilities in Developing Innovation-Related Capabilities. International Journal of Innovation Management , 459-478.

Etzioni, A. (1989). Humble Decision Making. Harvard Business Review , 67.

Fahy, J., \& Smithee, A. (1999). Strategic Marketing and the Resource Based View of the Firm. Academy of Marketing Science , 1-20.

Fielding, N. G., \& Fielding, J. L. (1986). Linking Data. Qualitative Research Methods. Beverly Hills: SAGE Publications.

Gary, L. (2001). Strategy as Process. Harvard Management Update , 1-3.

Gast, A. (2015, January 19th). Why business leaders should think like scientists. Retrieved January 24th, 2015, from AGENDA World Economic Forum: https://agenda.weforum.org/2015/01/why-business-leaders-should-think-likescientists/?utm_content=bufferb57a6\&utm_medium=social\&utm_source=facebook.c om\&utm_campaign=buffer

Ghemawat, P., \& Costa, J. (1993). The organizational tension between static and dynamic efficiency. Strategic Management Journal , 14 (8), 209-226.

Gibson, C. B., \& Birkinshaw, J. (2004). THE ANTECEDENTS, CONSEQUENCES, AND MEDIATING ROLE OF ORGANIZATIONAL AMBIDEXTERITY. Academy of Management Journal , 47 (2), 209-226.

Given, L. M. (2008). The Sage encyclopedia of qualitative research methods. Los Angeles: SAGE Publications.

González, E. M., Félix, R., Carrete, L., Centeno, E., \& Castaño, R. (2015). Green Shades: A Segmentation Approach Based on Ecological Consumer Behavior in an Emerging Economy. Journal of Marketing Theory and Practice , 23 (3), 287-302.

Grant, R. M. (2010). Contemporary Strategy Analysis Text and Cases (Seventh ed.). A John Wiley \& Sons, Ltd. Publication.

Grant, R. M. (1996). Prospering in Dynamically-competitive Environments: Organizational Capability as Knowledge Integration. Organization Science , 7 (4).

Greenhalgh, P. (2008). An Examination of Business Occupier Relocation Decision Making: Distinguishing Small and Large Firm Behaviour. Journal of Property Research , 107-126.

Gunther McGrath, R. (2013, June). Transient Advantage. Harvard Business Review , 62-70.

Haleblian, J., Finkelstein, \& Finkelstein, S. (1993). Top Management Team Size, CE0 Dominance, and Firm Performance: The Moderating Roles of Environmental Turbulence and Discretion. Academy of Management , 36 (4), 844-863.

Hammond, J. S., Keeney, R. L., \& Raiffa, H. (1998). Even Swaps: A rational Method for Making Trade-offs. Harvard Business Review , 2 (76), 137-150. 
Hammond, J. S., Keeney, R. L., \& Raiffa, H. (1998). The Hidden Traps in Decision Making. Harvard Business Review , 143-167.

Haris, I. (2012). Determinant Factors of Decision Making Process in Higher Education Institution (A Case of State University of Gorontalo, Indonesia). Global Journal of Management and Business Research , 12 (18), 32-40.

Hayashi, A. M. (2001, February). When to Trust your Gut. Harvard Business Review , 59-65.

Haytko, D. L., \& Baker, J. (2004). It's all at the mall: exploring adolescent girls' experiences. Journal of Retailing , 67-83.

Henderson, R., \& Cockburn, I. (1994). Measuring Competence? Exploring Firm Effects in Pharmaceutical Research. Strategic Management Journal , 1-36.

Hernández-Yáñez, M. L. (2011). Prioridades, políticas y educación superior. Revista de la Educación Superior , 40 (157).

Hitt, M. A., Gimeno, J., \& Hoskisson, R. E. (1998). Current and Future Research Methods in Strategic Management. Organizational Research Methods , 1 (1), 6-44.

Hughes, S., \& White, R. (2005). Improving Strategic Planning and Implementation in Universities through Competitive Intelligence Tools: A Means to Gaining Relevance. Journal of Higher Education Outreach and Engagement, 10 (3), 39-52.

Itami, H. (1987). Mobilizing Invisible Assets. Cambridge, MA: Harvard University Press.

Jick, T. D. (1979). Mixing Qualitative and Quantitative Methods: Triangulation in Action. Administrative Science Quarterly , 24, 602-611.

Jones, B. D. (1999). Bounded Rationality. Annual Review of Political Science , 2, 297 321.

Joyce, K. e. (2003). Lessons for employers from Fortune's "100 best". Business Horizons , 77-84.

Kaplan, R. S., \& Norton, D. P. (2004, March). Strategy Maps. Strategic Finance , 27-35.

Kaplan, R., \& Norton, D. (2001). The strategy focused organization. Harvard Business Press.

Kaplan, R. S., \& Norton, D. (2000, Sep-Oct). Having Trouble with your strategy? Then Map It. Harvard Business Review .

Khefacha, I., \& Belkacem, L. (2009). Decision-Making Models and University Governance: Experience From Tunisian Public Higher Education Establishments. 5360.

Kirp, D. L. (2003). Shakespeare, Einstein, and the Bottom Line: The Marketing of Higher Education. Cambridge, MA: Harvard University Press.

Knapp, K. R. (2001). The Balanced Scorecard: Historical Development and Context, As Developed by Robert Kaplan \& David Norton. Foundations of Management , 1-12. 
Kotter, J. P. (1990). What leaders really do. Harvard Business Review , 103-111.

Krosnick, J. A., \& Presser, S. (2010). Question and Questionnaire Design. In P. V. Marsden, \& J. D. Wright, Handbook of Survey Research (2nd ed., p. 903). Emerald Publishing Group Limited.

Lado, A. A., Boyd, N. G., Wright , P., \& Kroll, M. (2006). Paradox and Theorizing within the Resource-Based View. Academic of Management Review, 31 (1), 115-131.

Langley, A. (1995). Between "Paralysis by Analysis" and "Extinction by Instinct". Sloan Management Review, 36 (3), 63 - 77.

Lavie, D. (2006). Capability reconfi guring: An analysis of incumbent responses to technological change. Academy of Management Review , 31, 153-174.

Levinthal, D., \& March, J. G. (1993). Myopia of learning. Strategic Management Journal , 14, 95-112.

Lippman, S. A., \& Rumelt, R. P. (1982). Uncertain Imitability: An Analysis of Interfirm Differences in Efficiency under Competition. 13 (2), 418-438.

Lockett, A. (2005). Edith Penrose's Legacy to the Resource-Based View. Managerial and Decision Economics , 26, 83-98.

Lockett, A., \& Thompson, S. (2001). The resourcebased view and economics. Journal of Management, 27, 723-754.

Lockett, A., Thompson, S., \& Morgenstern, U. (2009). The development of the resource-based view of the firm: A critical appraisal. International Journal of Management Reviews , 11, 9-28.

Lorange, P., \& Vancil, R. F. (1976). How to Design a Strategic Planning System. Harvard Business Review , 74-81.

Lubatkin, M. H., Simsek, Z., Ling, Y., \& Veiga, J. F. (2006). Ambidexterity and Performance in Small-to Medium-Sized Firms: The Pivotal Role of Top Management Team Behavioral Integration. Journal of Management .

March, J. G. (1991). Exploration and Exploitation in Organizational Learning. Organizational Science , 2 (1), 71 - 87.

March, J. G. (1978). Bounded Rationality, Ambiguity, and the Engineering of Choice. The Bell Journal of Economics , 9, 587-608.

March, J. G., \& Cohen, M. D. (1974). Leadership and Ambiguity. Boston: Harvard Business School Press.

Marginson, S. (2006). Globalisation, the idea of a university and its ethical regimes. Higher Education Management and Policy, 19 (1), 31-46.

Martinez, M. (2008, September). Competencies and Higher Education Policy Analysts. Education Policy, 623-639.

Mauborgne, R., \& Kim, W. C. (2005). Blue Ocean Strategy. Harvard Business School Press. 
Meng, F. (2015). Problems Caused by the Management of Multi-Campus University in China. Higher Education of Social Science , 8 (4), 60-63.

Miller, H. (1994). The management of change in universities. Buckingham, England: SRHE \& The Open University Press.

Mills , J., Platts, K., \& Bourne, M. (2003). Applying resource-based theory. Methods, outcomes and utility for managers. International Journal of Operations \& Production Management , 148-166.

Mintzberg, H. (1994, January-February). The Fall and Rise of Strategic Planning. Harvard Business Review , 107-114.

Mintzberg, H. (1988, Summer). Crafting strategy. McKinsey Quarterly , 71-90.

Mintzberg, H. (1976, July-August). Planning on the left side and managing on the right. Harvard Business Review , 49-60.

Mintzberg, H. (1987). The Strategy Concept I: Five Ps for Strategy. California Management Review , 11-24.

Mintzberg, H. (1987). The Strategy Concept II: Another Look at Why Organizations Need Strategies. California Management Review , 25-32.

Mintzberg, H., Raisinghani, D., \& Theoret, A. (1976). The structure of 'unstructured' decision processes. Administrative Science Quarterly , 21, 246-275.

Mintzberg, H., \& McHugh, A. (1985, June). Strategy Formation in an Adhocracy. Administrative Science Quarterly, 160-197.

Mintzberg, H., \& Waters, J. A. (1985). Of Strategies, Deliberate and Emergent. Strategic Management Journal , 6, 257-272.

Nelson, R. R., \& Winter, S. G. (1982). An Evolutionary Theory of Economic Change. Cambridge, MA, USA: HARVARD UNIVERSITY PRESS .

Oliver, C. (1997). Sustainable Competitive Advantage: Combining Institutional and Resource Based Views. Strategic Management Journal, 18 (9), 697-713.

O’Mara, M. A. (1999). Strategic Drivers of Location Decisions for Information-age Companies. Journal of Real Estate Research , 365-386.

Okendu, T. (2008). Leadership and Strategic change in a Church-based institution: An exploratory qualitative case study. Capella University. Minneapolis: ProQuest.

O'Reilly, C., \& Tushman, M. (2007, March). Ambidexterity as a Dynamic Capability: Resolving the Innovator's Dilemma. Research Papers Series , 1-62.

Ozer, M. (2005). Factors which influence decision making in new product evaluation. European Journal of Operational Research , 784-801.

Payne, J. W., Samper, A., Bettman, J. R., \& Luce, M. F. (2008). Boundary Conditions on Unconscious Thought in Complex Decision Making. Psychological Science , 19 (11), 1118-1123.

Penrose, E. T. (1959). The Theory of the Growth of the Firm. New York: John Wiley. 
Peters, T. J., \& Waterman Jr., R. H. (1982). In Search of Excellence: Lessons from Americas Best Run Companies. HarperCollins.

Peters, T. (2005). Re-imagina. Madrid: Pearson Education.

Peterson, G. (2013, March 12). Forbes.com. Retrieved from http://www.forbes.com/sites/garypeterson/2013/03/12/cutting-rowe-wont-curebest-buy/

Pfeffer, J., \& Davis-Black, A. (1986). Administrative Succession and Organizational Performance: How Administrator Experience Mediates the Succession Effect. Academy of Management, 26 (1), 72-83.

Pitelis, C. (2005). Edith Penrose, Organisational Economics and Business Strategy: An Assessment and Extension. Managerial and Decisions Economics , 26, 67-82.

Porter, M. E. (2008). The Five Competitive Forces that Shape Strategy. Harvard Business Review. , 1-18.

Porter, M. E. (2008, January). The Five Competitive Forces that Shape Strategy. Harvard Business Review , 78-93.

Porter. (1996, Nov-Dec). What is Strategy? Harvard Business Review , 61-78.

Porter. (1980). Competitive Strategy . Free Press.

Porter, M. E. (1979, March-April). How competitive forces shape strategy. Harvard Business Review , 137-145.

Porter, M. E., \& Lawrence, B. W. (2011, Ene-Feb). La Creación de Valor Compartido. Harvard Business Review / Edición en Español , 32-49.

Pounder, J. S. (2001). "New leadership" and university organisational effectiveness: exploring the relationship. Leadership \& Organization Development Journal , 281-290.

Priem, R. L., \& Butler, J. E. (2001b). Is the resource-based "view" a useful perspective for strategic management research? Academy of Management Review , 26 (1), 22-40.

Priem, R. L., \& Butler, J. E. (2001a). Tautology in the resource-based view and the implications of externally determined resource value: further comments. Academy of Management Review , 26 (1), 57-66.

Raisch, S., \& Birkinshaw, J. (2008). Organizational Ambidexterity: Antecedents, Outcomes, and Moderators. Journal of Management, 375-409.

Ramos, D. (2012, August 06). Universidades privadas ya concentran la tercera parte de la matrícula en México. Retrieved 2016, from Animal Político: http://www.animalpolitico.com/2012/08/universidades-privadas-ya-concentranla-tercera-parte-de-la-matricula-en-mexico/

Ramsden, P. (1998). Learning to Lead in Higher Education. Routledge: Psychology Press.

Rao, P. (2014). A Resource-Based View of the "Best Companies in Mexico: A Multiplecase Design Approach. SAM Advanced Management Journal , 12-47. 
Rindfleish, J. M. (2003). Segment Profiling: reducing strategic risk in higher education management. Journal of Higher Education Policy and Management, 25 (2), 147-159.

Robio-Oca, J. (2006). LA POLÍTICA EDUCATIVA Y LA EDUCACIÓN SUPERIOR EN MÉXICO. Secretaría de Educación Pública. México: Fondo de Cultura Económica.

Rossman, G., \& Rallis, S. F. (2012). Learning in the field: An introduction to qualitative research. (3. ed., Ed.) Thousand Oaks, CA, USA: SAGE.

Rubin, H. J., \& Rubin, I. S. (2012). Qualitative Interviewing: The Art of Hearing data. Illinois: SAGE.

Slaughter, S., \& Rhoades, G. (2004). Academic Capitalism and the New Economy Markets, State, and Higher Education. Baltimore: The Johns Hopkins University Press.

Schulze, P., Heinemann, F., \& Abedin, A. (2008, Jan). BALANCING EXPLOITATION AND EXPLORATION Organizational Antecedents and Performance Effects of Ambidexterity. Academy of Management Annual Meeting Proceedings , 1 - 6.

Shafir, E., Simonson, I., \& Tversky, A. (1993). Reason-based choice. Cognition , 49, 1136.

Shattock, M. (2003). Managing Successful Universities. Buckingham: SRHE and OU Press.

Shivakumar, R. (2014). How to Tell Which Decisions are Strategic. California Mangement Review , 56, 78-97.

Silas-Casillas, J. C. (2005, January). Realidades y tendencias en la educación superior privada mexicana. Perfiles Educativos , 27 (109-110).

Sieber, J. E., \& Lanzetta, J. T. (1964). Conflict and Conceptual Structure as Determinants of Decision-Making Behavior. Journal of Personality , 622-641.

Simon , H. (1957). Models of Man. New York: Wiley.

Simon, H. A. (1959). Theories of Decison Making in Economics and Behevioral Science. The American Economic Review , 253-283.

Simon, H. (1947). Administrative Behavior. New York : Macmillan.

Smith, J. E., Carson, K. P., \& Alexander, R. A. (1984). Leadership: It Can Make a Difference. Academy of Management, 27 (4), 765-776.

Smith, Z. A., \& Wolverton, M. (2010). Higher Education Leadership Competencies: Quantitatively Refining a Qualitative Model. Journal of Leadership \& Organizational Studies , 17 (1), 61-70.

Spendlove, M. (2007). Competencies for effective leadership in higher education. International Journal of Educational Management. , 21, 407-417.

Steiner, G. (1979). Strategic Planning: What Every Manager Must Know. New York: Free Press.

Tavernier, K. (2005). Relevance of Strategic Management for Universities. Tijdschrifr voor Economie en Management, L (5), 1-16. 
Tecnológico-de-Monterrey. (2014, September 05). Our purpose: Education that transforms lives. Retrieved January 30, 2015, from Tecnológico de Monterrey: http://www.itesm.mx/wps/wcm/connect/ITESM/Tecnologico+de+Monterrey/Engl ish/About+Us/Transforming+lives/Our+purpose/?cache=none

Teece, D. (2007). Explicating dynamic capabilities: the nature and microfoundations of (sustainable) enterprise performance. Strategic Management Journal , 28, 13191350 .

Teece, D. J., Pisano, G., \& Shuen, A. (1997). Dynamic Capabilities and strategic Management. Strategic Management Journal, 18 (7), 509-533.

Teece, D. J., Pisano, G., \& Shuen, A. (1997). Dynamic Capabilities and Strategic Management. Strategic Management Journal, 18 (7), 509-533.

Teece, D., Pisano, G., \& Shuen, A. (1990). Firm Capabilities, Resources and the Concept of Strategy. Economic Analysis and Policy Working Paper EAP , 1-28.

Thompson, A., Peteraf, M., \& Gamble, J. (2010). Crafting \& Executing Strategy: The Quest for Competitive Advantage Concepts and Cases (17th Edition ed.). USA: Mc Graw Hill.

Thompson, J. E., \& Harrison, J. (2000). Competent managers? The development and validation of a normative model using the MCI standards. Journal of Management Development. , 19, 836-853.

Tierney, W. G. (1988). Organizational Culture in Higher Education: Defining the Essentials. The Journal of Higher Education, 59 (1), 2-21.

Timberlake, G. R. (2004). Decision-making in multi-campus higher education institutions. The Community College Enterprise , 91-99.

Tushman, M. L., Smith, W., Wood, R. C., Westermann, G., \& O'Reilly III, C. A. (2004). Innovation Streams and Ambidextrous Organizational Designs: On Building Dynamic Capabilities.

Van Maanen, J. (1979). Reclaiming qualitative methods for organisational research: a preface. Administrative Science Quarterly , 520-526.

van Manen, M. (1990). Researching lived experience: Human science for an action sensitive pedagogy. Albany: State University of New York Press.

Volberda, H. W. (2004). Crisis in Strategy:fragmentation, integration or synthesis. European Management Review , 35-42.

Volberda, H., \& Lewin, A. (2003). Co-evolutionary dynamics within and between firms: From evolution to coevolution. Journal of Management Studies , 40, 2111-2136.

Vogel, R., \& Güttel, W. H. (2013). The Dynamic Capability View in Strategic Management: A Bibliometric Review. Internationa Journal of Management Reviews, 426-446.

Voigt, T. (2014). Ambidextrous leadership in innovation management processes: Exploring the dynamics of opening and closing leadership behaviors at different levels 
of the Stage-Gate model. Master Degree Thesis Dissertation, University of Twente, Enschede.

Washburn, J. (2005). The Corporate Corruption of Higher Education. New York: Basic Books.

Wassener, B. (2015, November 15). New Term for Emerging Economies Is Suggested. Retrieved July 24, 2016, from The New York Times: http://www.nytimes.com/2010/11/16/business/global/16eagles.html?_r=1

Waterman, R. (1990). Adhocracy: The Power to Change. W.W. Norton.

Webb , E. J., Campbell, D. T., Schwart, R. D., \& Sechrest, L. (1966). Unobtrusive Measures: Nonreactive Research in the Social Sciences. . Chicago: Chicago: Rand McNally.

Wernerfelt, B. (1984). A resource-based view of the firm. Strategic Management Journal , 5, 171-180.

Wernerfelt, B. (1995). The Resource-Based View of the Firm: Ten Years After. Strategic Management Journal , 16, 171-174.

Wester, K. L., \& Borders, D. L. (2014). Research Competencies in Counseling: A Delphi Study. Journal of Counseling \& Development, 92, 447-458.

Wilson, T. D., \& Schooler, J. W. (1991). Thinking Too Much: Introspection Can Reduce the quality of Preferences and Decisions. Journal of Personality and Social Psychology, 60 (2), 181-192.

Wind, J. Y., \& Main, J. (1998). Driving Change. New York: The Free Press.

Winter, S. (2003). Understanding Dynamic Capabilities. Strategic Management Journal , 24, 991-995.

Wolverton, M., Ackerman, R., \& Holt, S. (2005). Preparing for leadership: what academic department chairs need to know.t. Journal of Higher Education Policy and Management, 27 (2), 227-238.

Worley, C. G., Hitchin, D. E., \& Ross, W. L. (1996). Integrated Strategic Change . Virginia: Reading Mass: Addison Wesley.

Wrapp, E. (1967). Good Managers Don't Make Policy Decisions. Harvard Business Review. , 1-22.

Xie, J., \& Zhang, C. (2009). Multi-campus university management model re-explore. Yichun College Journal , 183-184.

Yielder , J., \& Codling, A. (2004). Management and Leadership in the Contemporary University. Journal of Higher Education Policy and Management , 26 (3), 315-328.

Yin, R. K. (1994). Case study research Design and methods. Thousand Oaks, CA: SAGE Publications, Inc.

Zermeño-Casas, L. O., Armenteros-Acosta, M. d., Sologaistoa-Guangorena, A. G., \& Villanueva-Armenteros, Y. (2014). Competencias directivas: su identificación para instituciones de educación superior. Revista Global de Negocios , 2 (4), 25-42. 